
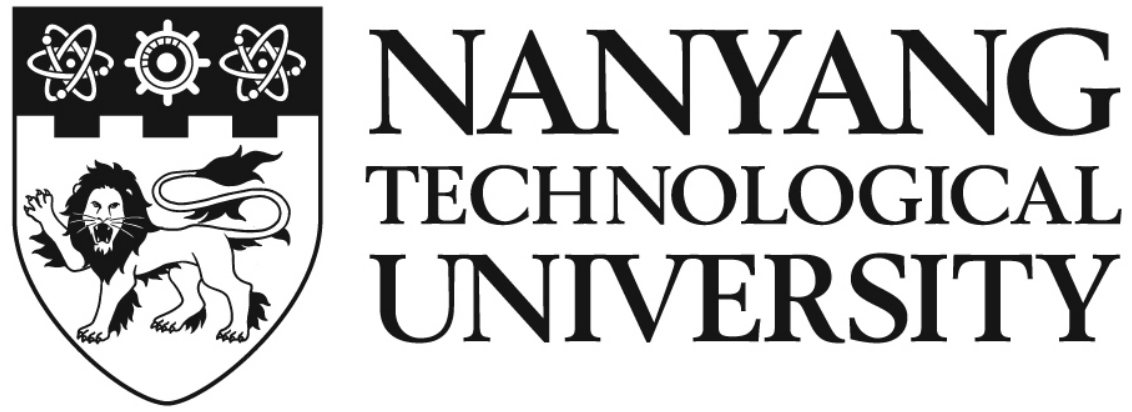

UNIVERSITY

FOURIER-TRANSFORM K'P METHOD FOR MODELING ELECTRONIC STRUCTURES AND OPTICAL PROPERTIES OF LOW DIMENSIONAL HETEROSTRUCTURES

善

\title{
ZHAO QIUJI
}

莕 SCHOOL OF ELECTRICAL \& ELECTRONIC ENGINEERING 2012 


\section{FOURIER-TRANSFORM K·P METHOD FOR MODELING ELECTRONIC STRUCTURES AND OPTICAL PROPERTIES OF LOW DIMENSIONAL HETEROSTRUCTURES}

\section{ZHAO QIUJI}

School of Electrical \& Electronic Engineering

A thesis submitted to the Nanyang Technological University in partial fulfillment of the requirement for the degree of Doctor of Philosophy 


\section{ACKNOWLEDGMENTS}

First and foremost, I would like to express my sincere appreciation to my academic supervisor, Prof. Mei Ting, for his great patience, kind support, and precious time, throughout my $\mathrm{PhD}$ study. I am greatly indebted to him for his invaluable academic guidance, stimulating suggestions, and critical comments, without which my achievements would not have been possible.

I am also very grateful to Prof. Zhang Dao Hua who has provided me great assistances during my PhD study. My gratitude also goes to Dr. Oka Kurniawan for his valuable advices on my research projects.

My sincere gratefulness extends to my senior Dr. Xu Chengdong, who trained me to operate experimental equipments and shared his valuable skills with me. Colleagues in NTU Photonics Lab I and Nanophotonics Lab have offered me great helps and valuable discussions. I would like to give special thanks to $\mathrm{Mr}$. $\mathrm{Li}$ Yicen, Mr. Wang Libo, Mr. Feng Chenggang, Ms. Wang Qian, Ms. Lee Shuhying, Dr. Tran Nguyen Vy Quynh, Dr. Stevanus Darmawan, and Dr. Landobasa Yosef Mario A. L. Tobing.

I also would like to acknowledge lab-technicians in Photonics Lab I, Photonics Lab II, and Nanophotonics Lab: Ms Yvong Yee, Mr. Yong Kim Lam, Ms. Low Poh Chee, Ms. Debbie Yang, Dr. Ng Tsu Hau, Dr. Yang Ming, and Mr. Chia Keng Yee for their assistances in lots of technical and administration-related matters.

Last but not least, I would like to thank my beloved family, for their love, support, and encouragement without reservation during my pursuit of academic degrees. 


\section{TABLE OF CONTENTS}

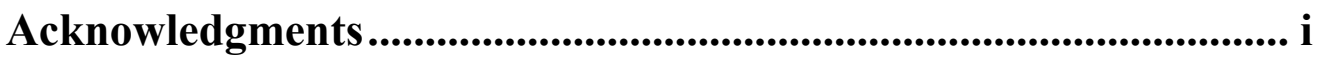

Table of Contents ............................................................................ ii

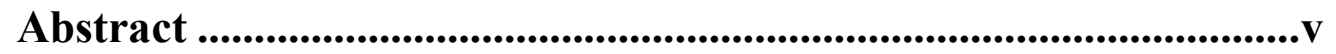

List of Figures .................................................................................. viii

List of Tables ........................................................................................ xii

List of Abbreviations .......................................................................... xiv

Chapter 1 Introduction ......................................................................................1

$1.1 \quad$ Background ....................................................................................................

1.1.1 Low dimensional heterostructures ......................................................

1.1.2 $\mathrm{k} \cdot \mathrm{p}$ computation for low dimensional heterostructures............................

1.2 Motivations .........................................................................................................

$1.3 \quad$ Objectives................................................................................................................9

$1.4 \quad$ Significant contributions ..................................................................................9

$1.5 \quad$ Thesis organization .............................................................................................12

Chapter 2 Literature review........................................................................14

2.1 Theoretical fundamentals of band modeling .............................................14

2.1.1 Band structures of semiconductors ..................................................14

2.1.2 Reciprocal lattices and Brillouin zone …………............................... 16

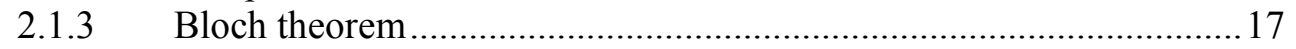

2.1.4 Envelope function theory ............................................................. 18

2.2 Basic theory of strain and piezoelectricity..................................................19

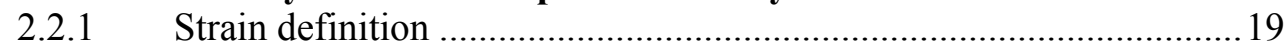

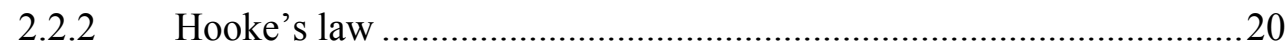

2.2.3 Strain and symmetry properties of crystals ..........................................21

2.2.4 Strain effect on band structure ........................................................23

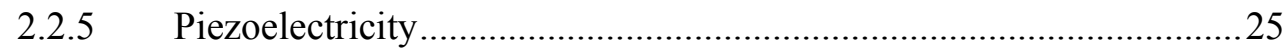

2.3 Computation of strain and piezoelectricity ...............................................27

2.3.1 Strain calculation for QWs............................................................27

2.3.2 Strain calculation models for QDs .....................................................27 


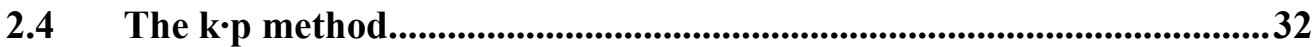

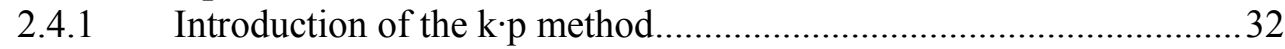

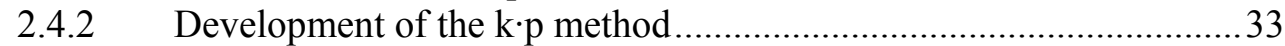

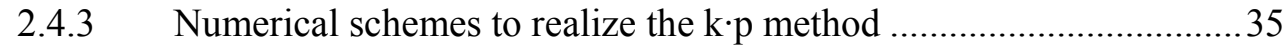

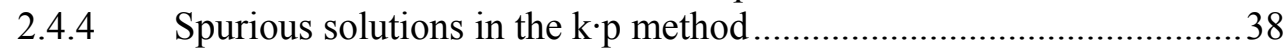

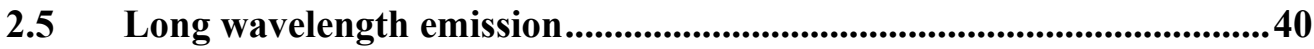

2.6 Interband optical gain spectrum of QWs ...............................................42

2.6.1 General formulation of the optical gain spectrum ............................4 43

2.6.2 Determination of the quasi-Fermi level ..........................................4

2.6.3 Interband transition matrix elements ................................................46

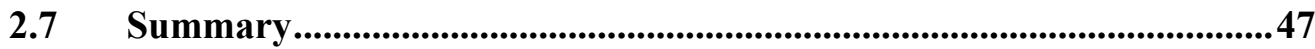

Chapter 3 Formulation and computation of FTM for QDs ............49

3.1 Formulation of FTM for QDs ......................................................49

3.3 Implementation of FTM for QDs .............................................................55

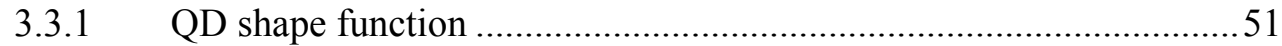

3.3.2 Constructing the Hamiltonian matrix in the Fourier domain ...............52

3.4 FTM computations for isolated QDs.........................................................58

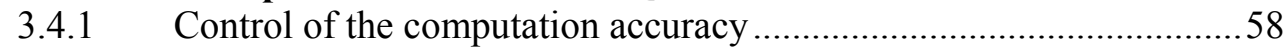

3.4.2 Further reduction of the memory consumption ................................6 68

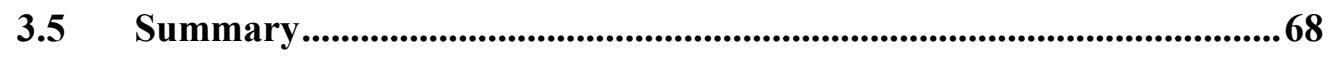

Chapter 4 Electronic structures of QDs ...........................................70

4.1 Influence of dimension and strain .......................................................

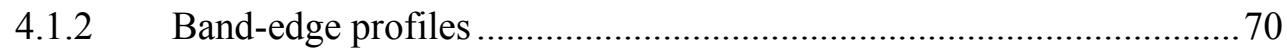

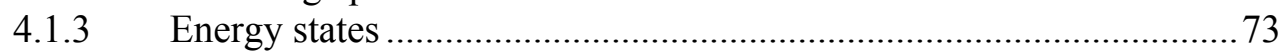

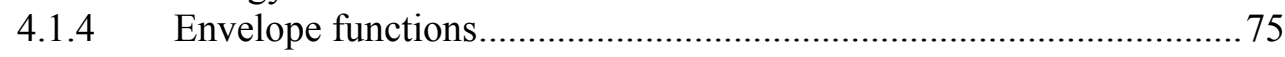

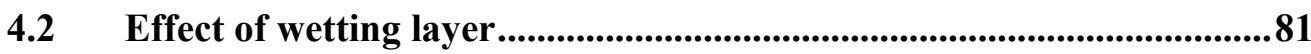

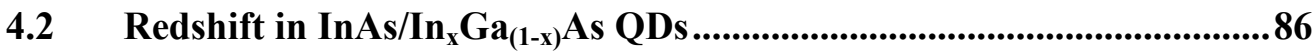

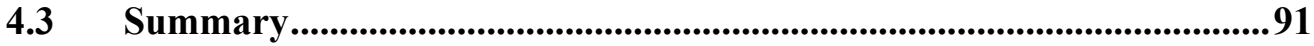

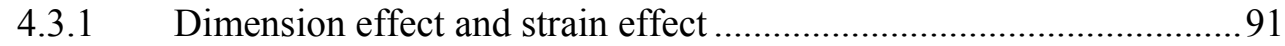

4.3.2 Effect of the wetting layer.............................................................. 92

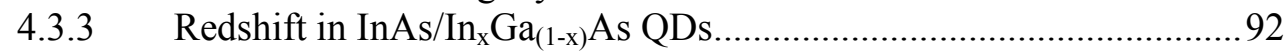

Chapter 5 Elimination of spurious solutions ..................................94

$5.2 \quad$ FTM with the BF operator ordering ..........................................................94 


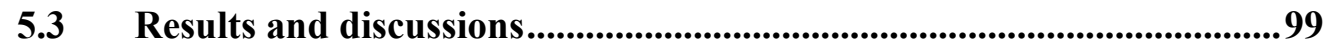

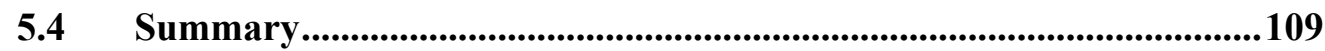

Chapter 6 Optical properties of QWs...........................................111

6.1 Application of FTM results on optical gain of III-Nitride QWs..........111

6.1.1 Interband transition matrix for III-Nitride QWs ............................. 112

6.1.2 Optical gain of $\mathrm{GaN}-\mathrm{Al}_{0.3} \mathrm{Ga}_{0.7} \mathrm{~N}$ QWs ........................................ 114

6.1.3 Optical gain of InGaN/InAlGaN QWs with a zero internal field .....116

6.2 Interband transition matrix elements for zinc blende QWs ................121

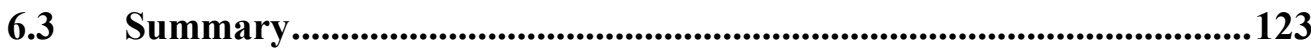

Chapter 7 Conclusions and recommendations ...............................125

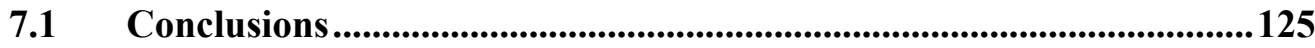

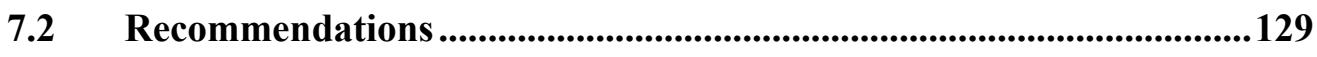

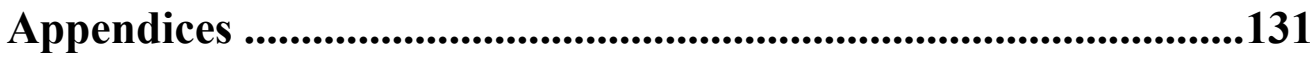

Author's Publications.....................................................................146

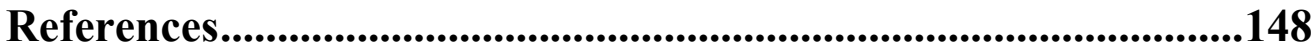




\begin{abstract}
Low dimensional heterostructures are promising candidates for the applications of optoelectronic devices, therefore, they have drawn increasing research efforts. In order to bestow the development of related experimental applications, various numerical methods have been developed to explore the electronic structures and the optical properties of heterostructures. The Fouriertransform $\mathrm{k} \cdot \mathrm{p}$ method $(\mathrm{FTM})$ has opened an entirely new way to investigate the band structures of quantum wells (QWs). Consequently, the work contained in this thesis is directly towards the implementation of FTM to study the electronic structures and the optical properties of low dimensional heterostructures.

In this thesis, an original meshless version of FTM for quantum dots (QDs) has been formulated and then been applied to calculate the electronic structures of isolated QDs successfully. The formulation of a simple and neat Hamiltonian matrix in the Fourier domain was fulfilled using the orthogonality of plane wave exponential functions and the truncation of higher Fourier harmonics. The geometric shape effect of QDs was introduced into the Hamiltonian matrix using the analytical Fourier transform of QD shape functions, and thus the formulation of the meshless programming. The critical factors related to computation accuracy were also discussed. In order to achieve enough computation accuracy in the calculation for isolated QD structures, the periodic length was employed as three times the largest dimension of the QD structure, and a higher order of Fourier truncation was selected for QDs with sharper geometric structures.

Subsequently, the meshless FTM was adopted to investigate the electronic structures of QDs. This study shows that strain causes band mixing effect reduced.
\end{abstract}


Strain also leads to variation in the shape and location of probability density functions (PDFs), and thus a weaker confinement to carriers. PDFs of $\mathrm{HH}$ and LH are more adapted to the variation of confining potential than that of $\mathrm{CB}$ due to heavier effective mass. Strain effect is more prominent for sharper pyramidal QDs due to stress concentration. This thesis shows that the wetting layer has to be considered in the calculation for very flat QDs. The wetting layer widens the potential well and, therefore, reduces the energy band-gap. The size of PDFs is magnified and the position of PDFs is driven towards the wetting layer. Both the band energies and the contour drawing of PDFs illustrate that the influence of the wetting layer on electronic structures is mainly from the region underneath the bottom of the dot region. Mechanism of redshift in $\operatorname{InAs} / \mathrm{In}_{\mathrm{x}} \mathrm{Ga}_{(1-\mathrm{x})} \mathrm{As}$ QDs has also been studied. Increasing In composition reduces the compressive strain, and thus the emergence of the redshift. The influence of the thickness of the lower confining layer cannot be compared to that of In composition. $0 \leq x \leq 0.33$ and a thicker lower confining layer can be adopted to avoid size fluctuations of QDs and weaken the thermal quenching due to the waning of band discontinuity.

The Burt-Foreman (BF) operator ordering was then incorporated into FTM to resist spurious solutions, which is one of the main challenges of using the $\mathrm{k} \cdot \mathrm{p}$ method. The BF operator ordering is effective to prevent spurious solutions in the six-band calculation, and spurious solutions in the $\mathrm{CB}$ met in the eight-band calculation can also be easily screened away in the inborn cut-off step (truncation of higher Fourier harmonics) in FTM. A wild-spreading spectrum of the Fourier series of the envelope function can be regarded as the signature of spurious solutions, which can be prevented by truncating the higher order of Fourier frequencies. 
Finally yet importantly, FTM results were employed to investigate optical properties of QWs successfully, further verifying the validity of the FTM calculation. Based on the band energy dispersions, the quasi-Fermi level and the interband transition momentum matrix elements were determined, and thus the optical gain spectrum. The interband transition matrix elements for both six-band and eight-band computation were given out by integrating the Fourier series of envelope functions conveniently. 


\section{LIST OF FIGURES}

Figure 1-1 The bulk structure and low dimensional heterostraucutres (upper part) and the corresponding DOSs of electrons (lower part). Figure reproduced

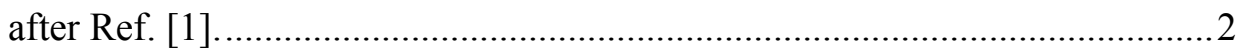

Figure 1-2 A layer material with a lattice constant a grown on a substrate with a lattice constant $\mathrm{a}_{\mathrm{s}}$ : (a) unstrained and (b) strained. Figure reproduced after Ref. $[22]$.

Figure 1-3 Shcemetic of the SK epitaxial growth model with a self-assembled pyramidal QD structure as the illustration example. 3

Figure 2-1 Brillouin zone for semiconductor crystals: (a) the zinc blende structure and (b) the wurtzite structure. Figure reproduced after Ref. [44].

Figure 2-2 Band diagram of semiconductor crystals: (a) the zinc blend structure, and (b) the wurtzite structure. Figure reproduced after Ref. [44] .................. 16

Figure 2-3 Deformation of an extendible string: (a) unstretched, and (b) stretched. Figure reproduced after Ref. [105]. 19

Figure 2-4 Band structure in strained zinc blende semiconductors: (a) band structure without strain, (b)band structure under the biaxial tension, and (c) band structure under the biaxial compression. Here $k_{\|}$is the $\mathrm{k}$ value in the plane of the layers, and $k_{\perp}$ is the $\mathrm{k}$ value along the axis perpendicular to the layers. Figure produced after Ref. [22]. .23

Figure 2-5 Optical transitions. (a) Optical transitions between CB and VBs, and (b) The Fermi-Dirac distributions $\mathrm{f}_{\mathrm{c}}$ and $\mathrm{f}_{\mathrm{v}}$. Figure cited after Ref. [22].

Figure 3-1 Geometric model of a pyramidal QD, where the dot is buried in the periodic box, $\mathrm{d}_{\mathrm{x}}, \mathrm{d}_{\mathrm{y}}$, and $\mathrm{d}_{\mathrm{z}}$ are the dimensions of the periodic box along $\mathrm{x}, \mathrm{y}$, and $\mathrm{z}$ directions, respectively.

Figure 3-2 Influence of the periodic length on the strain distribution of the isolated InAs/GaAs pyramidal QDs ( $\mathrm{f}=0$ ). $\mathrm{R}$ is the criterion cited after Ref. [124], a-d denote the strain distribution calculated using the Fourier transform method with different periodic lengths. 61

Figure 3-3 Influence of the periodic length on the strain distribution of the isolated InAs/GaAs half-truncated pyramidal QDs $(\mathrm{f}=0.5)$. $\mathrm{R}$ is the criterion cited after 
Ref. [124], a-d denote the strain distribution calculated using the Fourier transform method with different periodic lengths. The dark line represents the interface between the dot region and the wetting layer region.

Figure 3-4 Influence of the periodical length on strain distribution of the isolated InAs/GaAs pyramidal QDs ( $\mathrm{f}=0$ ) as wetting layer is included. a-d denote the strain distribution calculated using the Fourier transform method with different periodical lengths. The solid line represents the interface of the dot region and the wetting layer region.

Figure 3-5 Influence of the periodical length on strain distribution of the isolated InAs/GaAs half-trucnated pyramidal QDs $(\mathrm{f}=0.5)$ as wetting layer is included. a-d denote the strain distribution calculated using the Fourier transform method with different periodical lengths. The solid line represents the interface of the dot region and the wetting layer region. 64

Figure 3-6 Ground-state energies E1 and HH1 of the pyramidal QD with $\mathrm{L}_{\mathrm{x}}=136 \AA$, $\mathrm{L}_{\mathrm{y}}=136 \AA$ and $\mathrm{h}=60 \AA$ vary as a function of $\mathrm{L} / \mathrm{Lx}$ under different $\mathrm{N}_{\text {tr }}$. 66

Figure 3-7 Ground-state energies E1 and HH1 of the cuboidal QD with $a_{x}=a_{y}=a_{z}$ $=136 \AA$ vary as a function of $\mathrm{L} / \mathrm{a}_{\mathrm{x}}$ under different $\mathrm{N}_{\text {tr }}$.

Figure 4-1 Band-edge profile of the pyramidal QD example with $L_{x}=136 \AA$, $\mathrm{L}_{\mathrm{y}}=136 \AA$ and $\mathrm{h}=60 \AA$ along the [001] direction [Figure 4-1(a)] and the [100] direction [Figure 4-1(b)]. The dashed lines are the band-edge profiles before the strain modification and the solid lines are the band-edge profiles after the strain modification.

Figure 4-2 Band-edge profile of the half-truncated pyramidal QD example along the [001] direction [Figure 4-2(a)] and the [100] direction [Figure 4-2(b)]. The dashed lines are the band-edge profiles before the strain modification, and the solid lines are the band-edge profiles after the strain modification. .73

Figure 4-3 Ground-state energies of a series of truncated pyramidal QDs with (solid circles) and without (hollow circles) the strain consideration under different degree of height truncation .74

Figure 4-4 PDFs of the ground states of the pyramidal QD. (a) with the strain consideration and (b) without the strain consideration .76

Figure 4-5. PDFs of the ground states of the half-truncated pyramidal QD. (a) with the strain consideration and (b) without the strain consideration. .78 
Figure 4-6. Major PDF components of the three ground states of the pyramidal QD across the (001) plane and the (010) plane: (a) with the strain consideration and (b)without the strain consideration. The dash lines make up the pyramidal shape section. .79

Figure 4-7 Major PDF components of the three ground states of the half-truncated pyramidal QD across the (001) plane and the (010) plane: (a) with the strain consideration and (b)without the strain consideration. The dash lines make up the pyramidal shape section. 80

Figure 4-8 Geometric model of a truncated-pyramid InAs/GaAs QDW. The bottom dimension, the top dimension, and the height of dot are $12 \mathrm{~nm}, 12 \mathrm{f} \mathrm{nm}$, and $6(1-\mathrm{f}) \mathrm{nm}$, respectively. The thickness of the wetting layer is $0.5 \mathrm{~nm}$. $\mathrm{f}$ is the height truncation factor. 81

Figure 4-9 Ground-state energies E1 and HH1 of a series of truncated-pyramidal InAs/GaAs QDWs (solid lines) and a series of truncated-pyramidal QDs(dashed lines). .82

Figure 4-10 Strain components $\mathrm{e}_{11}$ and $\mathrm{e}_{33}$ of the half-truncated QD (dashed lines) and the half-truncated QDW (solid lines) along the [001] direction. 84

Figure 4-11 Band-edge profiles of the half-truncated QD (dashed lines) and the half-truncated QDW (solid lines) along the [001] direction 85

Figure 4-12 Major PDF components corresponding to ground-state energy E1 of the half-truncated QD and the half-truncated QDW [Figure 4-12(a)] and major PDF components corresponding to ground-state energy HH1 of the half-truncated QD and the half-truncated QDW [Figure 4-12(b)]. The black lines make up the shape section of the half-truncated QD and the halftruncated QDW 85

Figure 4-13 Initial lattice mismatch alters with the In composition $\mathrm{x}$ and the thickness of LCL 87

Figure 4-14 Band-edge profiles of the CB (dashed lines) and the VB (solid lines) after the strain modification (without the splitting between the $\mathrm{HH}$ band and the LH band) along the [001] direction (along the $\mathrm{z}$ axis) vary with a series of In composition $x(x=0,0.15,0.33,0.5)$, and the LCL thickness $t$ is fixed to $220 \mathrm{~nm}$ .88

Figure 4-15 Band-edge profiles of the CB (dashed lines), the HH band (solid lines) and the LH band (dotted lines) after the strain modification (with the splitting 
between the $\mathrm{HH}$ band and the LH band) along the [001] direction (along the $\mathrm{z}$ axis) vary with a series of LCL thicknesses $(\mathrm{t}=0 \mathrm{~nm}, 120 \mathrm{~nm}, 360 \mathrm{~nm}$, and $1000 \mathrm{~nm}$ ), and the In composition $\mathrm{x}$ is fixed to 0.15 . .89

Figure 4-16 Band-edge profiles of the CB (dashed lines) and the VB (solid lines) without the strain modification along the [001] direction (along the $\mathrm{z}$ axis) vary with a series of In compositions $\mathrm{x}(\mathrm{x}=0,0.15,0.33,0.5)$, and the LCL thickness $\mathrm{t}$ is fixed to $220 \mathrm{~nm}$. 90

Figure 4-17 Ground-state energies vary with a series of In compositions $\mathrm{x}(\mathrm{x}=0$, $0.15,0.33,0.5)$, and the LCL thickness $t$ is fixed to $220 \mathrm{~nm}$. Solid lines represent the case with strain, and dashed lines denote the case without strain.

Figure 5-1 Energy band diagrams of a $50 \AA$ InAs/GaAs QW obtained using the six-band Hamiltonian matrix varies with various order of Fourier truncation $\mathrm{N}_{\text {tr }}$ : (a) with the SYM operator ordering and (b) with the BF operator ordering. 100

Figure 5-2 Energy band diagrams of a $50 \AA$ InAs/GaAs QW obtained using the eight-band Hamiltonian matrix and the $\mathrm{BF}$ operator ordering varies with various order of Fourier truncation $\mathrm{N}_{\mathrm{tr}}$

Figure 5-3 Energy band diagram of a $50 \AA$ InAs/GaAs QW (calculated with the BF operator ordering) varies with different orders of Fourier truncation $\left(\mathrm{N}_{\mathrm{tr}}\right)$ and different mesh points $(\mathrm{N})$ : (a) the band energies at the center of Brillouin Zone $\left(\mathrm{k}_{\mathrm{t}}=0\right)$, and (b) the band energiess at the edge of Brillouin Zone $\left(\mathrm{k}_{\mathrm{t}}=0.2\right)$. The two dashed lines indicated the forbidden band region. 104

Figure 5-4 Fourier series $c_{n}$ of the envelope functions of a $50 \AA$ InAs/GaAs QW with the order of Fourier truncation (a) $\mathrm{N}_{\mathrm{tr}}=40$, (b) $\mathrm{N}_{\mathrm{tr}}=115$ and (c) $\mathrm{N}_{\mathrm{tr}}=$ 127. The computation is done with the $\mathrm{BF}$ operator ordering .The region composed by the two dashed lines indicates the Brillouin zone. 105

Figure 5-5 Energy band diagrams of a $18 \AA \times 18 \AA$ square GaN/AlN QWR obtained using the six-band Hamiltonian matrix varies with various order of Fourier truncation $\mathrm{N}_{\mathrm{tr}}$ : (a) with the SYM operator ordering and (b) with the BF operator ordering. 107

Figure 5-6 Major PDF components corresponding to the hole state of a $18 \AA \times 18 \AA$ square GaN/AIN QWR with (a) $\mathrm{N}_{\text {tr }}=10$ and (b) $\mathrm{N}_{\text {tr }}=11$, and the corresponding Fourier series $\mathbf{c}_{\mathbf{n}}$ of the envelope function with (c) $\mathrm{N}_{\mathrm{tr}}=10$ and 
(d) $\mathrm{N}_{\mathrm{tr}}=11$. The computation is done with the SYM operator ordering. The dashed boxes in (c) and (d) indicated the first Brillouin zones. 108

Figure 6-1 Spontaneous emission rate (on the right axis in arbitrary units) and optical gain spectrum (on the left axis in the unit of $1 / \mathrm{cm}$ ) of a $26 \AA / 62 \AA$ $\mathrm{GaN} / \mathrm{Al}_{0.3} \mathrm{Ga}_{0.7} \mathrm{~N}$ QW. The hollow circles denote the data numerically cited from Chuang's calculation. 115

Figure 6-2 Spontaneous emission rate (on the right axis in arbitrary units) and optical gain spectrum (on the left axis in the unit of $1 / \mathrm{cm}$ ) of a $50 \AA / 50 \AA$ GaN/ $\mathrm{Al}_{0.3} \mathrm{Ga}_{0.7} \mathrm{~N}$ QW. The hollow circles denote the data numerically cited from Chuang's calculation. 115

Figure 6-3 Optical gain spectra for the $\mathrm{In}_{0.05} \mathrm{Ga}_{0.85} \mathrm{~N} / \mathrm{Al}_{0.1} \mathrm{In}_{0.09} \mathrm{Ga}_{0.81} \mathrm{~N}$ QW with a well width of $35 \AA$ at the carrier concentrations $n=2 \times 10^{19}, 3 \times 10^{19}$, and $4 \times 10^{19}$ $\mathrm{cm}^{-3}$. Solid lines are for the TE polarization. Dashed lines are for the TM polarization.

Figure 6-4 Optical peak gain as a function of the well width (on the right axis in the unit of $1 / \mathrm{cm}$ ), and the corresponding position of photon energy (on the left axis in the unit of $\mathrm{eV}$ ) of $\mathrm{In}_{0.05} \mathrm{Ga}_{0.85} \mathrm{~N} / \mathrm{Al}_{0.1} \mathrm{In}_{0.09} \mathrm{Ga}_{0.81} \mathrm{~N} \mathrm{QW}$.

Figure 6-5 VB band energy of the $\mathrm{In}_{0.05} \mathrm{Ga}_{0.85} \mathrm{~N} / \mathrm{Al}_{0.1} \mathrm{In}_{0.09} \mathrm{Ga}_{0.81} \mathrm{~N}$ QWs with two different well widths: (a) $\mathrm{L}_{\mathrm{w}}=15 \AA$, and (b) $\mathrm{L}_{\mathrm{w}}=35 \AA$. The solid lines represent the $\mathrm{HH}$ state and the dashed lines describe the $\mathrm{LH}$ state. 121 


\section{LIST OF TABLES}

Table 3-1 Material parameters of InAs and GaAs. These parameters are cited after Ref.[109]. 58

Table 3-2 Dimension and periodic length of InAs/GaAs QDs. $f$ is the shape truncation factor. $\mathrm{f}$ are selected as $\mathrm{f}=0$ for $\mathrm{QD}$ structure $\mathrm{B}$, and $\mathrm{f}=0.5$ for the QD structure $\mathrm{D}$ (The dimension of the upper surface is $L_{x} f \times L_{y} f$ ).

Table 4-1 Ground-state energies E1 and HH1 of the half-truncated QD, the QDC, and the half-truncated QDW. 83

Table 5-1 Kane parameters for the six-band calculation. These parameters are from Ref. [73], in units of $\hbar^{2} / 2 m_{0}$ 100

Table 5-2 Material parameters used for the eight-band calculation. These parameter are cited after Ref. [73]. Except unit of Eg and $\Delta$ are eV, other parameters are in the unit of $\hbar^{2} / 2 m_{0}$ 102

Table 5-3 Material parameters used to investigate spurious solutions in the GaN/AlN QWRs. All of the material parameters are after Ref. [200].......... 108

Table 6-1 Material parameters used to compute optical properties of GaN/AlGaN QWs. All material parameters are after Ref.[182]

Table 6-2 The binary material parameters used to interpolate $\mathrm{Al}_{\mathrm{x}} \mathrm{In}_{\mathrm{y}} \mathrm{Ga}_{\mathrm{z}} \mathrm{N}$. All of the material parameters are after Ref. [200].

116

Table 6-3 The bowing parameters for the wurtzite ternary nitride compounds. All of the material parameters are after Ref. [200]. 117 


\section{LIST OF ABBREVIATIONS}

\begin{tabular}{|c|c|}
\hline $\mathrm{BF}$ & Burt-Foreman \\
\hline BF FTM & $\begin{array}{l}\text { Fourier-transform } \mathrm{k} \cdot \mathrm{p} \text { method with the Burt-Foreman operator } \\
\text { ordering }\end{array}$ \\
\hline $\mathrm{CB}$ & conduction band \\
\hline $\mathrm{CL}$ & confining layer \\
\hline EMA & effective mass approximation \\
\hline FDM & finite difference method \\
\hline FEM & finite element method \\
\hline FFT & fast Fourier transform \\
\hline FTM & Fourier-transform $\mathrm{k} \cdot \mathrm{p}$ method \\
\hline DSO & density of state \\
\hline $\mathrm{HH}$ & heavy-hole \\
\hline LCL & lower confining layer \\
\hline LED & light emitting diode \\
\hline LH & light-hole \\
\hline PDF & probability density function \\
\hline QD & quantum dot \\
\hline QDC & $\begin{array}{l}\text { half-truncated pyramidal quantum dot with a cube just underneath } \\
\text { its bottom }\end{array}$ \\
\hline QDW & quantum dot with the wetting layer \\
\hline QW & quantum well \\
\hline QWR & quantum wire \\
\hline SK & Stranski-Krastanov \\
\hline SYM & symmetrized \\
\hline SYM FTM & $\begin{array}{l}\text { Fourier-transform } \mathrm{k} \cdot \mathrm{p} \text { method with the symmetrized operator } \\
\text { ordering }\end{array}$ \\
\hline UCL & upper confining layer \\
\hline VB & valence band \\
\hline VCEL & vertical cavity-surface emitting laser \\
\hline
\end{tabular}




\section{CHAPTER 1}

\section{INTRODUCTION}

This chapter presents an overview of the thesis to introduce the background information, the motivations and the objectives of this study, summarizes the major contributions of this research, and finally briefs the organization of this thesis.

\subsection{Background}

\subsubsection{Low dimensional heterostructures}

Low dimensional heterostructures family is created by quantum wells (QWs), quantum wires (QWRs), and quantum dots (QDs), where motions of carriers are restricted by the quantum confinement. This leads to the discrete energy states and sharper density of states (DOSs) than the bulk structures [1, 2], as illustrated in Figure 1-1. Thanks to their interesting physical properties, low dimensional heterostructures are widely applied in the field of optoelectronics, such as heterostructure lasers [3-8], heterostructure light emission diodes (LEDs) [9-14], and single photon devices [15-20]. A considerable amount of available experimental and theoretical investigations on low dimensional heterostructures supplement each other, and bring about the flourishing vision in the field of optoelectronics.

QWs are usually fabricated by the epitaxial technique [21]. An epitaxial layer with a natural lattice constant $a$ is typically grown on the top of a substrate 
with a lattice constant $a_{s}$, as shown in Figure 1-2. Up to a certain critical thickness, the epitaxial layer will try to assume that the lattice constant (in the plane of the layer) matches the lattice constant of the substrate. As a result, the epitaxial layer is in the biaxial tension or the compression. The initial lattice mismatch is defined as,

$$
e_{0}=\frac{a_{s}-a}{a}
$$

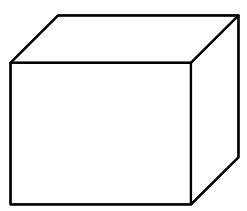

Bulk

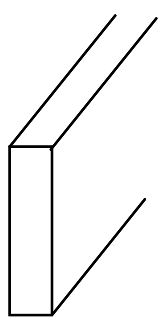

QW

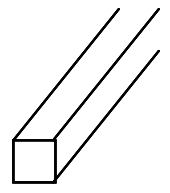

QWR

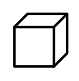

QD
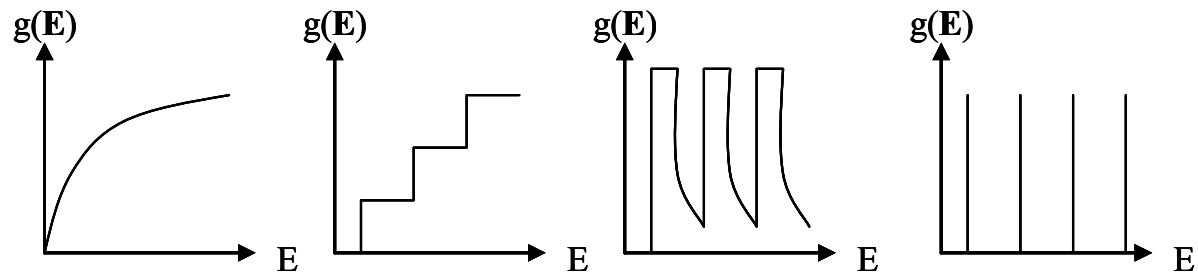

Figure 1-1 The bulk structure and low dimensional heterostraucutres (upper part) and the corresponding DOSs of electrons (lower part). Figure reproduced after Ref. [1].

(a) Unstrained
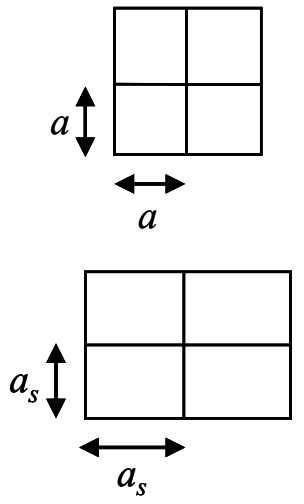

(b) Strained

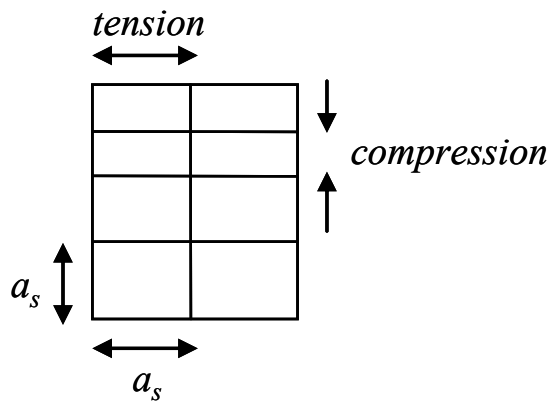

Figure 1-2 A layer material with a lattice constant a grown on a substrate with a lattice constant $a_{s}$ : (a) unstrained and (b) strained. Figure reproduced after Ref. [22]. 
Self-assembled QDs in the application of optoelectronics are usually fabricated by the Stranski-Krastanov (SK) epitaxial growth model [23, 24]. Taking the pyramidal QD shown in Figure 1-3 as an example, an approximated 1-2ML wetting layer, whose material is usually the same as that in the dot region, is grown on a substrate followed by a spontaneous coherent dot island formation process. Then, a deposition of an additional GaAs material, which usually uses the same material as the substrate, is adopted to cover the dot island. The deposition of the thin wetting layer leads to lattice mismatch at the interface of the wetting layer and the substrate, and the associated strain energy increases rapidly as the wetting layer thickens. As a critical thickness of the wetting layer is created, the formation of a dot island occurs to relieve the strain energy [25].

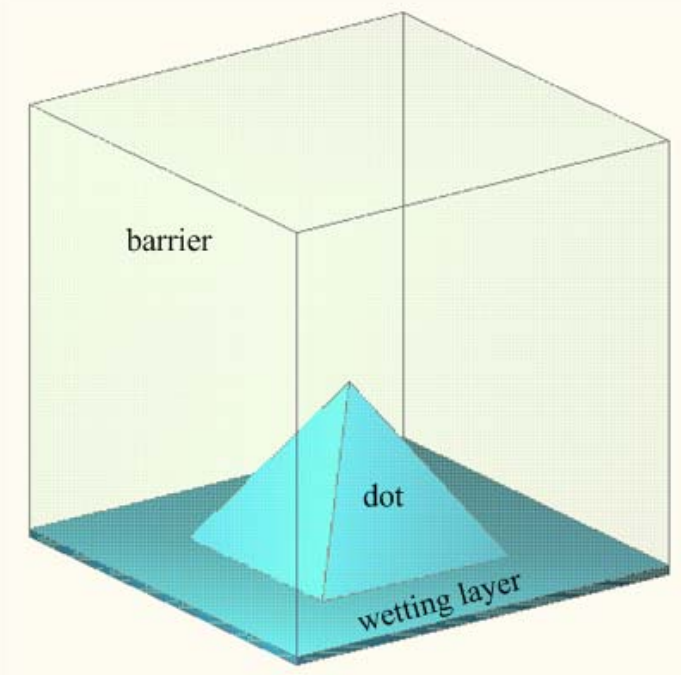

Figure 1-3 Shcemetic of the SK epitaxial growth model with a self-assembled pyramidal QD structure as the illustration example.

\subsection{2 $k \cdot p$ computation for low dimensional heterostructures}

Since low dimensional heterostructures have attracted increasing research efforts due to their interesting physical properties and promising applications, it is 
crucial to understand their electronic structures for the optoelectronics applications. With the high performance of computer hardware, the development of theory, and the efficient computation algorithms, several calculation models have been applied for calculating electronic structures of heterostuctures. The empirical tight-binding method [26-29], the empirical pseudopotential method [30-33], the single-band effective mass approximation (EMA) [34-36], and the multiband $\mathrm{k} \cdot \mathrm{p}$ method [3744] are the most frequently-used models. However, the multiband $\mathrm{k} \cdot \mathrm{p}$ calculation model [37-44] is one of the most widely adopted approaches among them due to its simplicity and accuracy for modeling the band structures near the Brillouin zone center $\Gamma$ point [38]. For the energy states near the $\Gamma$ point, application of symmetry on the $\mathrm{k} \cdot \mathrm{p}$ theory can reduce the undetermined constants, and thus give rises to much simpler operation in the calculation $[45,46]$.

The biggest challenge to adopt $\mathrm{k} \cdot \mathrm{p}$ method is how to avoid spurious solutions, which are defined as those eigenvalues of the $\mathrm{k} \cdot \mathrm{p}$ Hamiltonian matrix situated in the band gap of heterostrucutres. The spurious solutions called wing-band (evanescent) solutions [47-50] do not contribute to effective-mass wavefunctions and thus been seldom discussed recently. On the contrary, the oscillatory spurious solutions that make the wavefunctions oscillating are troublesome and thus have been discussed frequently [51-53]. Since there is no consistent way to eliminate the oscillatory spurious solutions in the $\mathrm{k} \cdot \mathrm{p}$ theory at the present stage, the problem of spurious solutions is still very interesting and worth of in-depth studying.

The $\mathrm{k} \cdot \mathrm{p}$ Hamiltonian matrix for a heterostructure is position dependent, therefore, differential operator $\hat{k}_{i}$ and numerical methods are usually employed to solve that eigen energies and eigen wavefunctions. Besides the widely used finite element method (FEM) [54-57] and the finite difference method (FDM) [42, 43, 58, 
59], a Fourier transform-based eight-band k·p method (FTM) [60] has been developed with fast Fourier transform (FFT) to obtain the electronic structures of zinc blende QW superlattices successfully. Compared with FDM and FEM, FTM naturally avoids the cumbersome differential and integral processes done in FDM and FEM, respectively. Fourier transform technique also has the potential to resist spurious solutions by restricting the Fourier expansion of envelope functions to the small $k$ components [61], unlike FDM that may meet spurious solutions [62]. Therefore, a more comprehensive study of the electronic structures of low dimensional heterostructures using FTM is reasonable and imperative, even for the isolated QDs since the influence from other periods can be negligible as the periodic length is long enough.

\subsection{Motivations}

It is well known that strain plays an important role in the heterostructures grown with the epitaxial technique because of the different lattice constants between the epitaxial layer and the substrate. The strain makes significant influence on the band structure through modification with the hydrostatic components and the biaxial components [22]. Strain also induces the piezoelectric field, hence the piezoelectric potential further modifies electronic structures of heterostructures. In turn, strain affects the performance of optoelectronic device strongly. Prior to the investigation of the electronic structures of low dimensional heterostructures applied in optoelectronics, the strain distribution should be determined.

In the previous work [60], FTM for QW superlattices has been developed, and comparison between FTM and FDM also has been studied. It demonstrates 
that FTM is superior to FDM from several aspects, such as the predominance in reduction of Hamiltonian matrix size, no cumbersome differential process, and prominent for the heterostructures with abrupt interfaces. Meshing the 3D QD structure is necessary for methods like the FDM and FEM, where an interpolation scheme is usually troublesome. Inherently, FTM does not rely on space meshing, given that all inputs in Fourier domain are available. These advantages drive me to further develop the meshless FTM for QDs and then study the band structures of isolated QDs.

The influence of dimension on electronic structures has been investigated in many works $[34,35,39,41,59]$, and calculation including strain consideration has been reported widely $[27,29,39-42,55,59,63]$. Nevertheless, few k $\cdot \mathrm{p}$ works have discussed how the strain and the dimension of QDs affect band energies and wavefunctions. Consequently, the influences of dimension and strain on the band energies and the wavefunctions are necessary to be explored by adding the strain effect into the Hamiltonian matrix. In addition, strain engineering has been applied in $\mathrm{InAs} / \mathrm{In}_{\mathrm{x}} \mathrm{Ga}_{(1-\mathrm{x})} \mathrm{As}$ QDs to realize the long wavelength emission for telecommunications [64-66], whereas, some critical factors (e.g., size fluctuations, band mixing effect, and clear discussion about influence of strain and band discontinuity to redshift) have not been posted. Therefore, besides dimension effect and strain effect on electronic structures of QDs, it is also necessary to further discuss the redshift in InAs/ $\operatorname{In}_{\mathrm{x}} \mathrm{Ga}_{(1-\mathrm{x})} \mathrm{As}$ QDs.

In prior studies, the wetting layer is usually omitted or is seldom discussed thoroughly by considering the very thin thickness of the wetting layer in the past works [39, 41, 59, 67]. To the best of our knowledge, Lee et al [68] investigated wetting layer effect in a very shallow InAs/GaAs lens-shape QD using the atomic 
strain model and the empirical tight-banding model, while Melnik et al [69] formulated FEM to discuss wetting layer effect in a conical QD with the singleband Hamiltonian. The former describes the variation of wavefunctions due to the wetting layer effect quantitatively, and the latter mainly emphasize on the formulation of the computational model. Therefore, it is necessary to further examine the effect of wetting layer completely.

Emergence of spurious solutions is a long standing problem of the $\mathrm{k} \cdot \mathrm{p}$ theory in the representation of envelope function. The origin of oscillatory spurious solutions is still a controversial topic, although several methods to tackle this problem have been proposed accordingly. The Burt-Foreman(BF) operator ordering is one of the most common methods [70-74], and cutting off the components that generate spurious solutions is also widely adopted $[47,61,75,76]$. Nevertheless, even though the BF operator ordering is employed, several effective mass parameters have to be modified in order to overcome the residual spurious solutions occurring in the conduction band (CB) [71, 73], and uncertainty still exists even though relevant effective mass parameters can be set to arbitrary values with a change of Bloch basis $[71,77]$. In brief, there is no consistent way to eliminate spurious solutions in the $\mathrm{k} \cdot \mathrm{p}$ theory, and the problem of spurious solutions is still very interesting and worth of in-depth studying.

Optical properties of low dimensional heterostructures can provide valuable information for device design and theoretical interpretation to the performance of devices. Since the eigenvectors obtained from FTM can be used in escaping troublesome integration of envelope functions in the calculation of transition matrix elements (detail information will be introduced in Chapter 2), FTM results may simplify the optical gain computation to some extent, and thus it should be 
significant to illustrate that how FTM results can be employed in the optical gain computation. On the other hand, it is essential to further demonstrate the validity of FTM results through the optical gain computation. Consequently, implementation of FTM results to model optical properties of low dimensional heterostructures is also a part of this thesis.

GaN-based wide bandgap III-Nitride heterostructures have drawn much interest because of their promising application in the optoelectronic devices. For instance, blue to green LEDs [14, 78], white LEDs [79-82], and LDs in the ultraviolet wavelength region $[83,84]$. Most III-Nitride heterostructures adopted in the field of optoelectronic devices are binary and ternary III-Nitride compounds grown along the [0001] direction such as $\mathrm{InGaN} / \mathrm{GaN}, \mathrm{GaN} / \mathrm{AlGaN}$, and InGaN/AlGaN. One of the main challenges lies at the large internal piezoelectric field due to spontaneous polarization and strain-induced polarization, leading to the obvious decrease of the electron-hole wavefunction overlap, and thus small transition matrix elements and the quantum-confined Stark effect [85-89]. Recently, InAlGaN has been demonstrated as an effective way to control the internal piezoelectric field [90-93], Park et al [90] proposed an $\operatorname{In}_{\mathrm{x}} \mathrm{Ga}_{1-\mathrm{x}} \mathrm{N} / \mathrm{In}_{\mathrm{y}} \mathrm{AL}_{0.1} \mathrm{Ga}_{0.9-\mathrm{y}} \mathrm{N}$ QW structure with a zero internal field and illustrated that the photoluminescence of $\mathrm{In}_{\mathrm{x}} \mathrm{Ga}_{1-\mathrm{x}} \mathrm{N} / \mathrm{In}_{\mathrm{y}} \mathrm{Al}_{0.1} \mathrm{Ga}_{0.9-\mathrm{y}} \mathrm{N} \mathrm{QW}$ is enhanced in comparison with $\mathrm{InGaN} / \mathrm{GaN}$ QWs. To the best of our knowledge, there has been no reported investigation on the optical gain under lower carrier densities and its dependence on the well width in this interesting $\operatorname{In}_{\mathrm{x}} \mathrm{Ga}_{1-\mathrm{x}} \mathrm{N} / \mathrm{In}_{\mathrm{y}} \mathrm{Al}_{0.1} \mathrm{Ga}_{0.9-\mathrm{y}} \mathrm{N}$ QW structure. Therefore, these two aspects will be investigated in Chapter 6 as well.

In summary, the major motivation for this thesis lies in the potential of breaking a new ground in the area of the $\mathrm{k} \cdot \mathrm{p}$ computation for low dimensional 
heterostructures with FTM. There are unexplored avenues in applying FTM to areas, such as strain, wetting layer, spurious solutions in the $\mathrm{k} \cdot \mathrm{p}$ theory, and optical properties, etc. Consequently, further development of FTM to model electronic structures and optical properties is chosen as the topic of this thesis.

\subsection{Objectives}

The formulation of the meshless FTM for low dimensional heterostructures is derived with QDs as the illustration example. The effects of strain and wetting layer of QDs are investigated based on the calculation results of both band energies and probability density functions (PDFs). The BF operator ordering is then incorporated into FTM to deal with the spurious solution problem in the $\mathrm{k} \cdot \mathrm{p}$ theory. Finally, the optical properties of QWs are investigated taking advantages of the FTM results. As such this $\mathrm{PhD}$ project has the following main objectives:

1) Calculate of the strain distribution of QWs and QDs.

2) Present the principle of FTM with the symmetrized (SYM) operator ordering and demonstrate the realization of the meshless FTM with QD as an illustration example.

3) Investigate the effects of strain and wetting layer on the electronic structures of QDs using FTM with the SYM operator ordering.

4) Improve the previous FTM with the BF operator ordering for resisting spurious solutions.

5) Study the optical properties of III-Nitride QWs with FTM results

\subsection{Significant contributions}

The thesis demonstrates an original formulation of the meshless FTM and presents its application for a theoretical calculation on the electronic structures and 
the optical properties of low dimensional heterostructures. The major contributions of thesis are summarized as follows:

1) The Fourier transform technique is applied to the periodic positiondependent Hamiltonian, and a simple and neat expression of the Hamiltonian matrix in the Fourier domain is formulated using the orthogonality of exponential functions. The proposed FTM in this thesis is expected to be useful to investigate electronic structures and optical properties of other structures beyond the scope of this study. For instance, band structure of silicon, single nanowire, and other related materials. (See Chapter 3)

2) A kinetic Hamiltonian matrix in the momentum domain is formulated by entering the analytical Fourier transform of the QD shape function into the neat Hamiltonian matrix easily, realizing the meshless FTM for the first time. The formulation of strain Hamiltonian matrix is done by the convolution of the Fourier series of the strain components and the Fourier series of the QD shape functions. Therefore, an original meshless version of FTM for QD computation is developed. (See Chapter 3)

3) With the new proposed numerical method, the first and also the most important thing is to check its validity and accuracy. The critical factors causing numerical error such as the order of Fourier truncation is discussed. For the case of isolated QDs but not QD superlattices, the periodic length is another important factor to affect the computation accuracy. The periodic length is recommended no less than three times the largest dimension of a QD structures in order to guarantee sufficient accuracy. It is shown that 
FTM is competent for calculate electronic structures of isolated QDs. (See Chapter 3)

4) The meshless FTM is adopted to study the dimension effect and the strain effect on the electronic structures of the pyramidal QDs that are truncated to different heights. The ground-state energy variation shows that the electron state is the most sensitive to these two effects, and the strain effect on ground-state energies E1, HH1 and LH1 is more prominent for sharper QDs. Investigation shows that both band mixing between CB and VBs and band mixing between the heavy-hole (HH) band and the light-hole (LH) band are reduced due to the strain effect, whereas this effect is more prominent for non-truncated pyramidal QDs due to stress concentration. Among the three ground states, LH states are more weakly confined in the non-pyramidal QDs and shift to the tip of the pyramid due to the strain effect. (See Chapter 4)

5) A theoretical analysis of the wetting layer effect on the electronic structures of InAs/GaAs truncated-pyramidal QDs is carried out using FTM. Strain modified band-edge profiles indicates that the wetting layer widens the potential well inside the dot region. The wetting layer changes the groundstate energy significantly whereas modifies PDFs only a little. The main acting region of the wetting layer is just underneath the base of the dot. All of these variations are not affected by the shape of QDs. (See Chapter 4)

6) Mechanism of redshift in $\operatorname{InAs} / \mathrm{In}_{x} \mathrm{Ga}_{(1-\mathrm{x})} \mathrm{As}$ QDs is also investigated with FTM. Both the band-edge profiles and ground-state energies indicate that strain effect on redshift decreases as increasing of In component. In order to avoid the size fluctuations and the thermal quenching luminescence, the 
most proper range of In composition for remarkable redshift is $0 \leq x \leq 0.33$. (See Chapter 4)

7) To eliminate spurious solutions in the multiband $\mathrm{k} \cdot \mathrm{p}$ theory, the $\mathrm{BF}$ operator ordering was incorporated into FTM to formulate a new version of FTM. The performance is perfect for the six-band $\mathrm{k} \cdot \mathrm{p}$ calculation, and the spurious solutions in the $\mathrm{CB}$ met in the eight-band calculation can also be easily screened away in the inborn cut-off step in FTM, i.e. choosing a proper order of Fourier truncation. Truncating high-order terms of Fourier coefficients of the envelope function prevents the occurrence of a wildspreading spectrum of the Fourier expansion coefficients, which can be taken as the signature of spurious solutions. (See Chapter 5)

8) For the first time, the interband optical gain of QWs is investigated using the FTM results. The interband transition matrix elements for the six-band calculation and the eight-band calculation are given out. Optical gain calculated with FTM results is nearly the same as the reported one. (See Chapter 6)

\subsection{Thesis organization}

The thesis is organized into seven chapters.

Chapter 1 encompasses background information, motivations, objectives, and major contributions of this study.

Chapter 2 reviews relative literatures. It provides the concepts covered in this thesis and appraises the present state of the science about theoretical investigation of the electronic structures and the optical properties of low dimensional heterostructures. 
Chapter 3 presents the formulation of FTM for low dimensional heterostructures and the actual realization of the meshless FTM for QDs. Verification here shows that FTM is accurate and convenient for computing electronic structures of isolated QDs.

Chapter 4 investigates the effects of strain and wetting layer on electronic structures of QDs. Variations on band-edge profiles, band energies, and PDFs of QDs due to these effects are discussed in detail. Behind physical explanations are also described.

Chapter 5 builds on the work from chapter 3 by replacing the SYM operator ordering with the BF operator ordering and improves the previous FTM. The improved FTM is adopted to eliminate spurious solutions, which is common but troublesome in the $\mathrm{k} \cdot \mathrm{p}$ computation for QWs and QWRs. Control of spurious solutions of various kinds of structures is explored.

Chapter 6 applies the band energies and the envelope functions to figure out the interband transition matrix elements. The optical gain of III-Nitride QWs is also studied.

Chapter 7 concludes the thesis. It also gives the recommendations for improvements and further research directions for this research topic. 


\section{CHAPTER 2}

\section{LiTERATURE REVIEW}

In this chapter, the fundamentals of band theory and basic theory of strain and piezoelectricity are presented. Things related to $\mathrm{k} \cdot \mathrm{p}$ method, e.g, development of $\mathrm{k} \cdot \mathrm{p}$ method, numerical schemes to realize the $\mathrm{k} \cdot \mathrm{p}$ method, spurious solutions in the $\mathrm{k} \cdot \mathrm{p}$ method, are reviewed and discussed, as well as those widely used strain calculation methods for QWs and QDs. A brief introduction about computation of piezoelectric field and optical gain of QWs is also documented.

\subsection{Theoretical fundamentals of band modeling}

\subsubsection{Band structures of semiconductors}

Zinc blende primitive cell is composed with two penetrating face-center cubic sublattices and has one lattice constant (denoted as $a$ ), while the wurtzite primitive cell is a hexagonal prism with four atoms and has two lattice constants (denoted as $a$ and $c$ ) [44]. The Brillouin zone in Figure 2-1 is cited from Ref. [44]. Zinc blende structures are mostly composed by the group-IV elements, the group III-V compounds and a few II-VI compounds. Many of group II-VI compounds and a few group III-V compounds are wurtzite structures. In consideration of the application of the light emission, direct-gap zinc blende III-V compounds and wurtzite III-Nitride are the focus of attention in this thesis. 


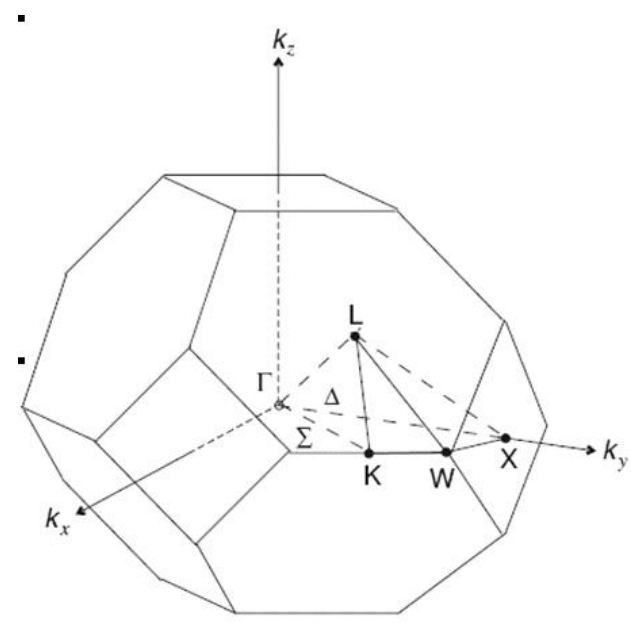

(a) Zinc blende

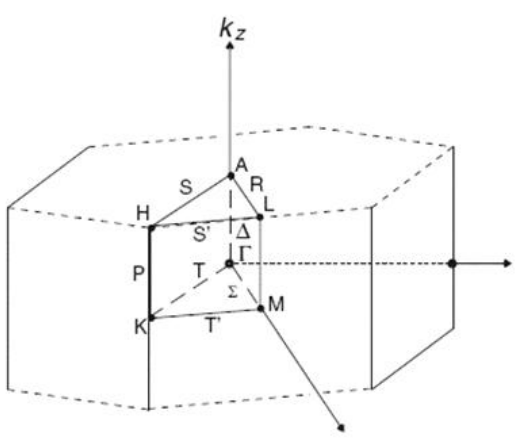

(b) wurtzite

Figure 2-1 Brillouin zone for semiconductor crystals: (a) the zinc blende structure and (b) the wurtzite structure. Figure reproduced after Ref. [44].

Different crystal structures lead to different band structures of zinc blende crystals and wurtzite crystals, which are shown in Figure 2-2. The most significant difference between Figure 2-2(a) and Figure 2-2(b) is the band gap. Larger bang gap of the wurtzite crystal leads to the ignorance of the band-mixing effect between $\mathrm{CB}$ and $\mathrm{VB}$, whereas the case of zinc blende crystal is on the contrary. Hence calculating electronic structures of zinc blende and wurtzite demands eightband Hamiltonian matrix and six-band Hamiltonian matrix, respectively. In addition, the crystal-filed splitting also has to be considered in wurtzite crystals, besides the spin splitting in zinc blende crystals. 


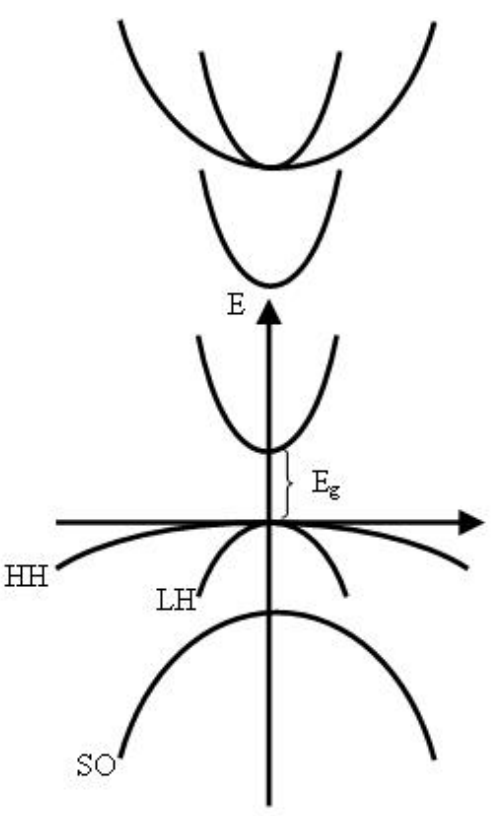

(a) Zinc blende

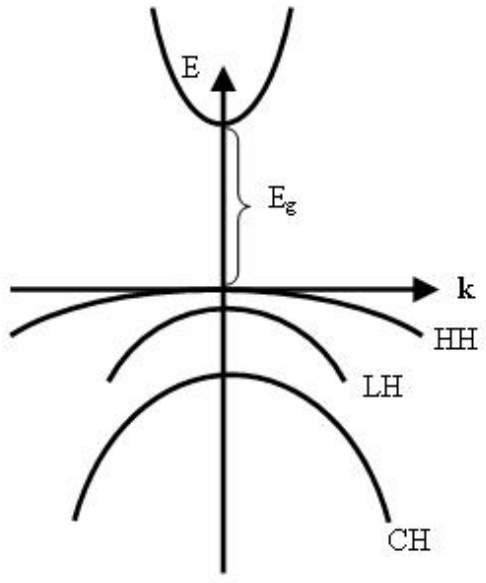

(b) Wurtzite

Figure 2-2 Band diagram of semiconductor crystals: (a) the zinc blend structure, and (b) the wurtzite structure. Figure reproduced after Ref. [44].

\subsubsection{Reciprocal lattices and Brillouin zone}

Any crystalline solids can be described in the fundamental concepts of Bravais lattice, which specifies the periodic array in which the repeated units of the crystal are attached, and the unit may be a single atom, a group of atoms, molecules, etc. The three-dimensional Bravais lattice consisting of entire points can be expressed as a position vector in the ordinary space, $\mathbf{R}=n_{1} \mathbf{a}_{1}+n_{2} \mathbf{a}_{2}+n_{3} \mathbf{a}_{3}$, where $\mathbf{a}_{1}, \mathbf{a}_{2}$ and $\mathbf{a}_{3}$ are three basis vectors, and $n_{1}, n_{2}$ and $n_{3}$ are any integers. It is also important to consider the reciprocal lattice in the reciprocal space, which is the lattice in which the Fourier transform of the spatial function of the ordinary lattice is represented. The reciprocal lattice primitive $\mathbf{b}_{\mathbf{j}}$ is defined by $\mathbf{a}_{\mathbf{i}} \cdot \mathbf{b}_{\mathbf{j}}=2 \pi \delta_{i j}(i, j=1,2,3)$, where $\delta_{i j}=\left\{\begin{array}{l}1, i=j \\ 0, i \neq j\end{array}\right.$ is Kronecker delta. Therefore the 
point vector in the reciprocal space is expressed as $\mathbf{G}=k_{1} \mathbf{b}_{1}+k_{2} \mathbf{b}_{2}+k_{3} \mathbf{b}_{3}$ accordingly [94], where $k_{1}, k_{2}$ and $k_{3}$ are any integers.

The first Brillouin zone (usually denoted as Brillouin zone) is the WignerSeitz cell of the reciprocal lattice, which is uniquely defined by planes that are the perpendicular bisectors of the vectors from the origin to the reciprocal lattice points [94]. A Brillouin zone is a unit cell in the reciprocal space. The importance of the Brillouin zone stems from the Bloch wave description of waves in a periodic crystal, so that electronic structures of the whole crystal can be completely characterized by those in a single Brillouin zone.

\subsubsection{Bloch theorem}

In the periodic crystalline, Bloch theorem $[95,96]$ can be applied to find the energy states $E(k)$ and wavefunctions $\psi(\mathbf{r})$ by solving the time-independent Schrödinger Equation,

$$
H \psi(\mathbf{r})=\left[\frac{-\hbar^{2}}{2 m_{0}} \nabla^{2}+V_{L}(\mathbf{r})\right] \psi(\mathbf{r})=E(\mathbf{k}) \psi(\mathbf{r}),
$$

where $V_{L}(\mathbf{r})=V_{L}(\mathbf{r}+\mathbf{R})$ is the time-independent periodic potential of crystal, $n$ is the band index, $m_{0}$ is the electron mass and $\hbar$ is reduced Planck's constant. The time-independent Hamiltonian is invariant under translation by lattice vectors $(\mathbf{r} \rightarrow \mathbf{r}+\mathbf{R})$, hence both $\psi(\mathbf{r})$ and $\psi(\mathbf{r}+\mathbf{R})$ are the solutions to Eq. (2.1). However, $\psi(\mathbf{r}+\mathbf{R})$ has to differ from $\psi(\mathbf{r})$ at most by a constant, which has to be a unit magnitude in order to avoid that the wavefunction may grow to infinite when the translation $\mathbf{R}$ was repeated indefinitely. Therefore, each electron wavefunction can be rewritten as a product of a plane wave and a function with periodicity of Bravais lattice, 


$$
\psi_{n}(\mathbf{r})=e^{i \mathbf{k} \cdot \mathbf{r}} u_{n, \mathbf{k}}(\mathbf{r})
$$

where $u_{n, \mathbf{k}}(\mathbf{r}+\mathbf{R})=u_{n, \mathbf{k}}(\mathbf{r})$ is the Bloch lattice function, and $\mathbf{k}$ is wave vector wandering through Brillouin zone.

\subsubsection{Envelope function theory}

Electronic structures of semiconductor crystals are understood by using envelope function approximation [47, 51, 97-104], which has been proved efficient and relatively reliable compared with other more sophisticated approaches. Bastard [97] introduced a prescriptive envelope function method for heterostructure, which has been justified to be reasonable agreement with the results of semi-empirical tight-banding calculations. However, some problems may be encountered in some cases [104], since this approach postulates the envelope function equations rather than deriving them from a more fundamental microscopic Schrödinger equation. Burt [100] developed a conventional approximation to the exact envelope function representation, expanding the electron wavefunction as,

$$
\psi(\mathbf{r})=\sum_{j} F_{j}(\mathbf{r}) u_{j, 0}(\mathbf{r}),
$$

where $u_{j, 0}(\mathbf{r})$ are a complete set of orthogonal basis functions with period equal to the lattice constant, and $F_{j}(\mathbf{r})$ is the envelope function. The restriction of the plane wave expansion of $F_{j}(\mathbf{r})$ to the Brillouin zone is usually required to make sure the unique expansion in Eq. (2.3). 


\subsection{Basic theory of strain and piezoelectricity}

\subsubsection{Strain definition}

Strain specifies the state of deformation of a solid body, the simpler 1D case is taken up for consideration at first. Figure 2-3(a) shows an extendible string. After stretching [Figure 2-3(b)], an arbitrary point $P$ on the string moves to $P^{\prime}$, another point $Q$ near $P$ moves to $Q^{\prime}$. In studying of strain, actual displacement of points is not concerned, but their displacements relative to one and another. The strain of section PQ is defined as [105]:

$$
\frac{\text { increase in length }}{\text { origin in length }}=\frac{P^{\prime} Q^{\prime}-P Q}{P Q}=\frac{\Delta u}{\Delta x},
$$

where,

$$
\begin{aligned}
& P Q=\Delta x, \\
& P^{\prime} Q^{\prime}=\Delta x+\Delta u .
\end{aligned}
$$

The strain at $\mathrm{P}$ point is defined as,

$$
e=\lim _{\Delta x \rightarrow 0} \frac{\Delta u}{\Delta x}=\frac{d u}{d x}
$$

Consequently, the strain tensor can be described with,

$$
e_{i j}=\frac{\partial u_{i}}{\partial x_{j}}(i, j=1,2,3) \text {. }
$$

(a)

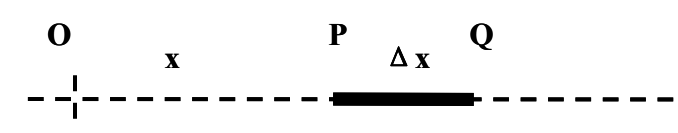

(b)

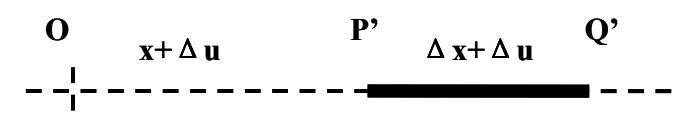

Figure 2-3 Deformation of an extendible string: (a) unstretched, and (b) stretched. Figure reproduced after Ref. [105]. 
With the symmetry consideration, final strain tensor is expressed as,

$$
e_{i j}=\frac{1}{2}\left(\frac{\partial u_{i}}{\partial x_{j}}+\frac{\partial u_{j}}{\partial x_{i}}\right), i, j=1,2,3
$$

\subsubsection{Hooke's law}

With Hooke's Law, the relationship between the strain $e$ and the stress $\sigma$ in $1 \mathrm{D}$ is,

$$
e=S \sigma
$$

or,

$$
\sigma=C e,
$$

with,

$$
C=1 / S,
$$

where $S$ is elastic compliance constant, and $C$ is elastic stiffness constants. When Hooke's Law is used for semiconductors, a homogenous strain is generated by a homogenous stress with the following relationship:

$$
e_{i j}=S_{i j k l} \sigma_{k l}(i, j, k, l=1,2,3),
$$

or,

$$
\sigma_{i j}=C_{i j k l} e_{k l}(i, j, k, l=1,2,3) .
$$

Both $S_{i j k l}$ and $C_{i j k l}$ have $3^{4}=81$ elements. With the symmetry property $\left(e_{i j}=e_{j i}\right)$ in the definition of strain, $S$ and $C$ possess the following symmetry property of,

$$
\begin{aligned}
S_{i j k l} & =S_{j i k l} \\
S_{i j k l} & =S_{i j l k} \\
C_{i j k l} & =C_{j i k l} \\
C_{i j k l} & =C_{i j l k}
\end{aligned} .
$$


Therefore, the 81 components are reduced to 36 components of $S$ and $C$. With the symmetry, the double suffix in strain and stress can be reduced to the single suffix running form 1 to 6 as following,

$$
\left(\begin{array}{lll}
e_{11} & e_{12} & e_{13} \\
e_{12} & e_{22} & e_{23} \\
e_{13} & e_{23} & e_{33}
\end{array}\right) \rightarrow\left(\begin{array}{lll}
e_{1} & e_{6} & e_{5} \\
e_{6} & e_{2} & e_{4} \\
e_{5} & e_{4} & e_{3}
\end{array}\right)
$$

and,

$$
\left(\begin{array}{lll}
\sigma_{11} & \sigma_{12} & \sigma_{13} \\
\sigma_{12} & \sigma_{22} & \sigma_{23} \\
\sigma_{13} & \sigma_{23} & \sigma_{33}
\end{array}\right) \rightarrow\left(\begin{array}{lll}
\sigma_{1} & \sigma_{6} & \sigma_{5} \\
\sigma_{6} & \sigma_{2} & \sigma_{4} \\
\sigma_{5} & \sigma_{4} & \sigma_{3}
\end{array}\right)
$$

\subsubsection{Strain and symmetry properties of crystals}

With the denotation of strain $e$ and stress $\sigma$ in Eq. (2.16) and Eq. (2.17), square array of compliance $S$ and stiffness $C$ can be rewritten as,

$$
\left(\begin{array}{llllll}
S_{11} & S_{12} & S_{13} & S_{14} & S_{15} & S_{16} \\
S_{21} & S_{22} & S_{23} & S_{24} & S_{25} & S_{26} \\
S_{31} & S_{32} & S_{33} & S_{34} & S_{35} & S_{36} \\
S_{41} & S_{42} & S_{43} & S_{44} & S_{45} & S_{46} \\
S_{51} & S_{52} & S_{53} & S_{54} & S_{55} & S_{56} \\
S_{61} & S_{62} & S_{63} & S_{64} & S_{65} & S_{66}
\end{array}\right),
$$

and,

$$
\left(\begin{array}{llllll}
C_{11} & C_{12} & C_{13} & C_{14} & C_{15} & C_{16} \\
C_{21} & C_{22} & C_{23} & C_{24} & C_{25} & C_{26} \\
C_{31} & C_{32} & C_{33} & C_{34} & C_{35} & C_{36} \\
C_{41} & C_{42} & C_{43} & C_{44} & C_{45} & C_{46} \\
C_{51} & C_{52} & C_{53} & C_{54} & C_{55} & C_{56} \\
C_{61} & C_{62} & C_{63} & C_{64} & C_{65} & C_{66}
\end{array}\right) .
$$

Strain is not a property of crystal but a response of a crystal to an influence. The influence may be a stress (elasticity) or an electrical field (piezoelectricity). In both cases, the magnitude and the direction of strain are determined by the magnitude 
and direction of the influence, as well as by the physical and the symmetry properties of crystal.

After the symmetry operation, the full matrix notation for the zinc blende crystals with cubic symmetry is [105],

$$
\left(\begin{array}{cccccc}
S_{11} & S_{12} & S_{12} & 0 & 0 & 0 \\
& S_{11} & S_{12} & 0 & 0 & 0 \\
& & S_{11} & 0 & 0 & 0 \\
& & & S_{44} & 0 & 0 \\
& & & & S_{44} & 0 \\
& & & & & S_{44}
\end{array}\right),
$$

and,

$$
\left(\begin{array}{cccccc}
C_{11} & C_{12} & C_{12} & 0 & 0 & 0 \\
& C_{11} & C_{12} & 0 & 0 & 0 \\
& & C_{11} & 0 & 0 & 0 \\
& & & C_{44} & 0 & 0 \\
& & & & C_{44} & 0 \\
& & & & & C_{44}
\end{array}\right) .
$$

The two lower left hand of the half of the array are omitted due to the symmetry of the two arrays. $\mu^{\prime}=0$ is Defined for the case of isotropic, while $\mu^{\prime} \neq 0$ is for the anisotropic case, where $\mu^{\prime}=C_{11}-C_{12}-2 C_{44}$. The full matrix notation for the wurtzite crystals with hexagonal symmetry is [105],

$$
\left(\begin{array}{cccccc}
S_{11} & S_{12} & S_{13} & 0 & 0 & 0 \\
& S_{11} & S_{13} & 0 & 0 & 0 \\
& & S_{33} & 0 & 0 & 0 \\
& & & S_{44} & 0 & 0 \\
& & & & S_{44} & 0 \\
& & & & & \frac{S_{11}-S_{12}}{2}
\end{array}\right),
$$

and, 


$$
\left(\begin{array}{cccccc}
C_{11} & C_{12} & C_{13} & 0 & 0 & 0 \\
& C_{11} & C_{13} & 0 & 0 & 0 \\
& & C_{33} & 0 & 0 & 0 \\
& & & C_{44} & 0 & 0 \\
& & & & C_{44} & 0 \\
& & & & & \frac{C_{11}-C_{12}}{2}
\end{array}\right) .
$$

\subsubsection{Strain effect on band structure}

Strain effect on the energy bands includes: 1) splitting HH and LH apart at $\mathrm{k}=0,2$ ) changing the separation between $\mathrm{CB}$ and $\mathrm{VB}$, and 3) breaking the even approximately isotropic. Taking a zinc blende structure as an example, the results of valence-band structure for $E_{H H}$ and $E_{L H}$ versus $k_{x}$ and $k_{z}$ for both compression and tension are shown in Figure 2-4.
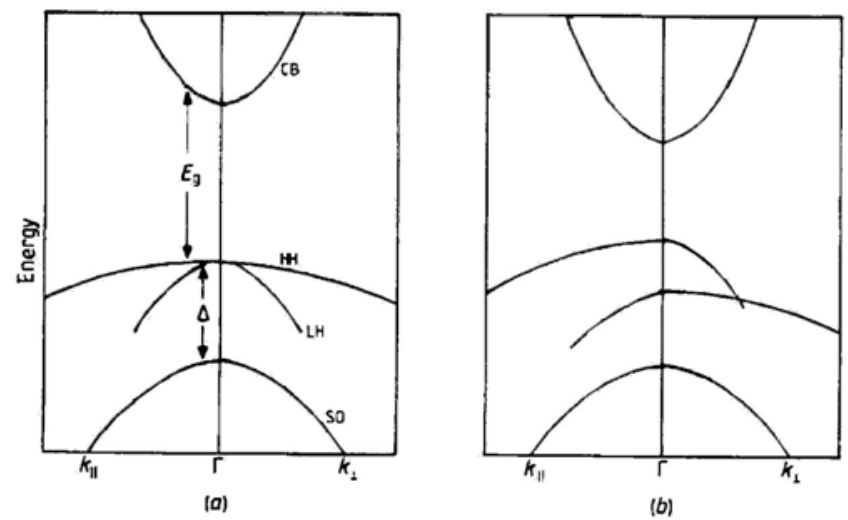

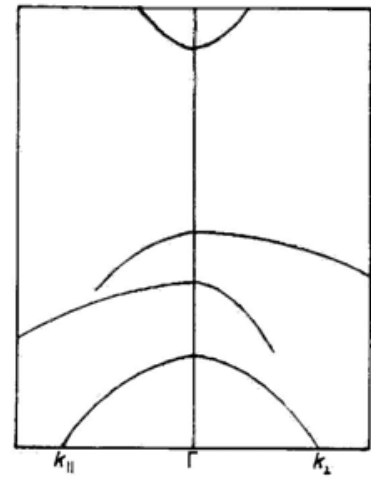

(c)

Figure 2-4 Band structure in strained zinc blende semiconductors: (a) band structure without strain, (b)band structure under the biaxial tension, and (c) band structure under the biaxial compression. Here $k_{\|}$is the $k$ value in the plane of the layers, and $k_{\perp}$ is the $k$ value along the axis perpendicular to the layers. Figure produced after Ref. [22].

After the modification by a finite and fixed strain, bands of zinc blende structures in the small $k$ space can be approximated quantitatively as [22],

$$
E_{C} \approx E_{g}+a_{c} e_{h},
$$




$$
\begin{aligned}
& E_{C-H H} \approx E g-P_{e}-Q_{e}, \\
& E_{C-L H} \approx E g-P_{e}+Q_{e}, \\
& P_{e}=a_{v} e_{h}, \\
& Q_{e}=b e_{b},
\end{aligned}
$$

where,

$$
\begin{aligned}
& e_{h}=e_{11}+e_{22}+e_{33}, \\
& e_{b}=e_{33}-\left(e_{11}+e_{22}\right) / 2 .
\end{aligned}
$$

The quantitative expression for band-edge profile after strain modification in wurtzite crystals is approximated in Ref. [106], which is omitted here.

With the basis functions $|S \uparrow\rangle,|S \downarrow\rangle,|X \uparrow\rangle,|X \downarrow\rangle,|Y \uparrow\rangle,|Y \downarrow\rangle,|Z \uparrow\rangle,|Z \downarrow\rangle$, the $4 \times 4$ strain Hamiltonian matrix corresponding to the four-band Kane Hamiltonian introduced previously is given by [107],

$$
\bar{D}=\left(\begin{array}{cccc}
s & x & y & z \\
a_{c} e_{h} & -i P_{0} \sum_{j} e_{1 j} k_{j} & -i P_{0} \sum_{j} e_{2 j} k_{j} & -i P_{0} \sum_{j} e_{3 j} k_{j} \\
i P_{0} \sum_{j} e_{1 j} k_{j} & m\left(e_{11}+\right. & \left.n e_{33}\right) & n e_{13} \\
i P_{0} \sum_{j} e_{2 j} k_{j} & n e_{12} & m\left(e_{11}+e_{33}\right) & n e_{23} \\
i P_{0} \sum_{j} e_{3 j} k_{j} & n e_{13} & n e_{23} & l e_{33}+ \\
& & & m\left(e_{11}+e_{22}\right)
\end{array}\right) \text {, }
$$

with,

$$
\begin{aligned}
& l=a_{v}+2 b \\
& m=a_{v}-b, \\
& n=\sqrt{3} d
\end{aligned}
$$

where $a_{c}, a_{v}, b$, and $d$ are the deformation-potential constants, $a_{c}$ describes the coupling of the CB to strain, $a_{v}, b$, and $d$ describe the coupling of the VB to strain. 


\subsubsection{Piezoelectricity}

With an external stress, both the zinc blende crystal and the wurtzite crystal develop an electric moment with a magnitude proportional to the applied stress $[105,108,109]$. The magnitude of the electric moment per unit volume is actually the polarization charge per unit area,

$$
P_{i}^{\text {strain }}=d_{i j k}^{\prime} \sigma_{j k}(i, j, k=1,2,3),
$$

where the strain induced polarization $P_{i}^{\text {strain }}$ is a vector with three components, $d_{i j k}^{\prime}$ is a third-rank tensor, which is named as the piezoelectric moduli. The last two suffixes in piezoelectric moduli can be reduced to the single suffix running from 1 to 6 as following,

$$
\left(\begin{array}{lll}
d_{i 11}^{\prime} & d_{i 12}^{\prime} & d_{i 13}^{\prime} \\
d_{i 21}^{\prime} & d_{i 22}^{\prime} & d_{i 13}^{\prime} \\
d_{i 31}^{\prime} & d_{i 32}^{\prime} & d_{i 33}^{\prime}
\end{array}\right) \rightarrow\left(\begin{array}{lll}
d_{i 1}^{\prime} & d_{i 6}^{\prime} & d_{i 5}^{\prime} \\
d_{i 6}^{\prime} & d_{i 2}^{\prime} & d_{i 4}^{\prime} \\
d_{i 5}^{\prime} & d_{i 4}^{\prime} & d_{i 3}^{\prime}
\end{array}\right) .
$$

With the above simple notation, the polarization $P_{i}$ can be rewritten as [109],

$$
P_{i}=d_{i k}^{\prime} \sigma_{k}=d_{i k}^{\prime} C_{k j} e_{j}=d_{i j} e_{j}(j, k=1,2,3, \cdots, 6),
$$

where $d_{i j}$ is the piezoelectric coefficient. After the symmetry operation in crystals, the full matrix notation of piezoelectric coefficients for the zinc blende crystals and wurtzite crystals are [105],

$$
\left(\begin{array}{cccccc}
0 & 0 & 0 & d_{14} & 0 & 0 \\
0 & 0 & 0 & 0 & d_{14} & 0 \\
0 & 0 & 0 & 0 & 0 & d_{14}
\end{array}\right),
$$

and

$$
\left(\begin{array}{cccccc}
0 & 0 & 0 & 0 & d_{15} & 0 \\
0 & 0 & 0 & d_{15} & 0 & 0 \\
d_{31} & d_{31} & d_{33} & 0 & 0 & 0
\end{array}\right),
$$

respectively. 
Substituting Eq. (2.36) into Eq. (2.35), the linear piezoelectric polarization (first order) for zinc blende crystals becomes,

$$
P_{i}^{\text {strain }}=d_{14} e_{j k}, j \neq k
$$

It has been shown that inclusion of the nonlinear piezoelectric effect (the second order) in zinc blende heterostructures can give a more accurate description of piezoelectric effect $[110,111]$.

Very recently, second order piezoelectric constants have been recommended by Paul [112]. The linear strain induced piezoelectric polarization (first order) for wurtzite crystals is expressed in the component form as,

$$
\begin{aligned}
& P_{1}^{\text {strain }}=d_{15} e_{13} \\
& P_{2}^{\text {strain }}=d_{15} e_{12} \\
& P_{3}^{\text {strain }}=d_{31} e_{11}+d_{31} e_{22}+d_{33} e_{33}
\end{aligned} .
$$

Except for the strain induced piezoelectric effect, wurtzite structures also exhibit a spontaneous polarization $\mathbf{P}^{s p}[113-115]$. The total polarization in wurtzite structures is,

$$
\mathbf{P}=\mathbf{P}^{s p}+\mathbf{P}^{\text {strain }} .
$$

The piezoelectric effect in zinc blende structures is negligible unless the epitaxial structure is grown along a less common direction such as the [111] direction [109]. But for the wurtzite crystals with hexagonal structures, piezoelectric effect can modify electronic structures significantly, and thus cannot be neglected. The large built-in piezoelectric field (potential) in wurtzite crystals mainly arises from the crystal symmetry and the large piezoelectric constants [116]. For instance, the piezoelectric constants $e_{31}$ and $e_{33}$ of wurtzite III-Nitride are several times larger than the piezoelectric constants of most III-V compounds. 


\subsection{Computation of strain and piezoelectricity}

\subsubsection{Strain calculation for QWs}

The strain in the pseudomorphic QWs is homogenous and confined in the slab. It can be calculated with simple analytical expression. Strain distribution in QWs can be obtained either with the stiffness constants [117] or with the Poisson's ratio and Young's modulus [118]. The former method is widely used due to its simple expressions and well determined stiffness constants, and thus been employed in this study. For the zinc blende QWs grown along the [001] direction, strain expressions are as [117],

$$
\begin{aligned}
& e_{12}=e_{13}=e_{23}=0, \\
& e_{11}=e_{22}=e_{0}, \\
& e_{33}=-\frac{2 C_{12}}{C_{11}} e_{0} .
\end{aligned}
$$

For the wurtzite QWs grown along the [001] direction, strain is calculated with [117],

$$
\begin{aligned}
& e_{12}=e_{13}=e_{23}=0, \\
& e_{11}=e_{22}=e_{0}, \\
& e_{33}=-\frac{2 C_{13}}{C_{33}} e_{0} .
\end{aligned}
$$

\subsubsection{Strain calculation models for QDs}

A handful of various calculation models have been demonstrated. Strainevaluation procedures can be divided into four broad types: 1) atomistic elasticity model $[31,37,39,119-122]$; 2) continuum mechanical model [39, 42, 55, 67, 119 , 121, 123-126]; and 3) more complex approaches [119] rely on Stillinger-Weber or 
Tersoff potentials. The first two kinds of procedures are more popular, and thus reviewed while the last case is omitted.

\subsubsection{Atomistic elasticity model}

Lattice mismatch caused atomic positions are relaxed to the minimum strain energy values [31]. Valence force field model are first used to function strain energy by Keating [120]. Displacements are evaluated at the atomistic level, therefore, this model provides greater accuracy than the continuum mechanical model at the QD edges, vertices, and interface of the well material and barrier material [124]. The elastic energy $V$ of crystal is summarized by Jiang and Singh [37] as,

$$
V=\frac{1}{4} \sum_{i j} \frac{3}{4} \frac{\left(d_{i j}{ }^{2}-d_{0, i j}{ }^{2}\right)^{2}}{d_{0, i j}{ }^{2}}+\frac{1}{2} \sum_{i} \sum_{j \neq k} \frac{3}{4} \frac{\beta_{i j k}\left(\mathbf{d}_{i j} \cdot \mathbf{d}_{i k}+d_{0, i j} d_{0, i k} / 3\right)^{2}}{d_{0, i j} d_{0, i k}},
$$

where $i$ runs over all the atomic sites, $j, k$ run over the nearest-neighbor sites of $i$, $d_{i j}$ is the vector joining the sites $i$ and $j, \boldsymbol{d}_{i j}$ is the length of bond, $d_{0, i j}$ is the corresponding equilibrium length in the binary constituents, and $\alpha_{i j}$ and $\beta_{i j k}$ are the bond stretching and bond bending constants, respectively.

Strain is found by minimization of the system energy using Eq. (2.47), which needs to solve a set of equations with $3 N$ variables, where $N$ is number of atoms. Consequently, atomistic techniques are computationally intensive, especially for those large systems. It also subjects to convergence problems and depends crucially on the quality of the inter-atomic potential model [124]. FEM is usually the numerical approach $[55,119,127]$ to realize this computation, and care is required to ensure the boundary conditions. In addition, the unavoidable awkward interpolation in FEM is frequently required to obtain results at a general coordinator [124]. 


\subsubsection{Continuum mechanical model}

Continuum mechanical model for calculating strain with uniformly latticemismatched inclusion is pioneered by Eshelby [128]. Based on this, a lot range of applications emerge, for example, a simple surface integration technique proposed by Downes $[118,123,124]$ and the Green's Function method used in Fourier domain [67] or real space $[125,126]$. The strong points of the continuum mechanical model lie at the powerful ability to treat large systems involving many QDs [124]. Andreev et al. [67] have developed an analytical expression based on the continuum mechanical theory, where the calculations are relatively quick and easy. However, the atomistic scale in atomistic elasticity model is lost, and most continuum mechanical models are under these assumptions [126]: 1) the elastic properties are linear; 2) identical elastic constants pertain to whole structure and the barrier parameters are usually adopted according to the Keyes' scaling rule [129]; 3) the well material is embedded in an infinite matrix. Elastic constants of barrier material are chosen because the following reason [124]: prior to the relaxation of the misfit strain, all materials possess the same lattice spacing, and the elastic constants of a highly strained QDs may be closer to the barrier material than to the well material in its relaxed state. Nevertheless, Andreev et al. [67] have put forward a treatment based on the perturbation theory to do the calculation with different elastic constants of the well and the barrier materials.

Integral, which is involved in the surface integration technique and Green's function method in real space with Gauss' theorem, are prone to meet the singularity. Even if the analytical expressions are given, the computation is still fussy due to those complex analytical expressions. Another defect of the surface 
integration technique is considering the anisotropic coefficient $\left(C_{11}-C_{12}\right) / 2 C_{44}$ equal to 1 instead of 0.5 in the III-V semiconductors.

For the Green's function model in Fourier domain, analytical solutions for strain in zinc blend QDs are produced by combining the elastic theorem and shape functions of QDs in the Fourier domain,

$$
e_{i j}=\frac{(2 \pi)^{3}}{d_{1} d_{2} d_{3}} \sum_{n_{1}, n_{2}, n_{3}} \tilde{e}_{i j}^{s}\left(\xi_{n}\right) \exp \left(i \xi_{n} \cdot \mathbf{r}\right)
$$

where $\xi_{n}=2 \pi\left(n_{1} / d_{1}, n_{2} / d_{2}, n_{3} / d_{3}\right)$ denotes the Cartesian coordinates in Fourier domain for particular harmonic component, and $\tilde{e}_{i j}^{s}$ term is the strain tensor in a single QD structure expressed in Fourier domain,

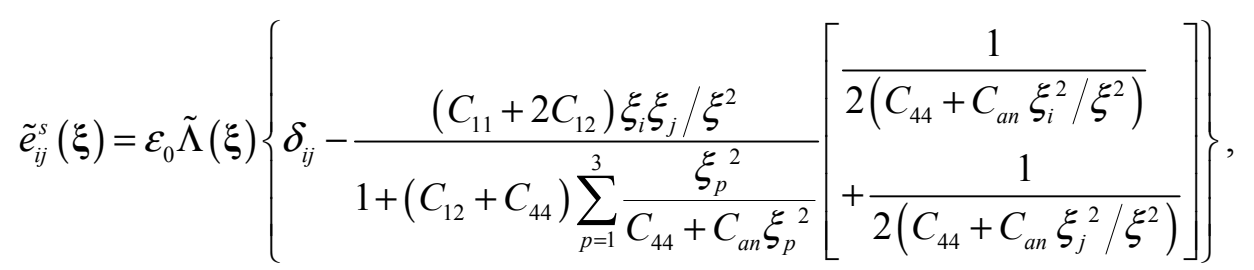

where $i, j=1,2,3$, and $\xi^{2}=\xi_{1}^{2}+\xi_{2}^{2}+\xi_{3}^{2}, C_{11}, C_{12}$ and $C_{44}$ are the three nonzero elastic compliance constant of the barrier-material with cubic symmetry, $\varepsilon_{0}=\left(a_{d}-a_{b}\right) / a_{d}$ is the initial lattice mismatch expressed with lattice constant of well material and barrier material. It can calculate both the isotropic case and the anisotropic case quit conveniently. A major advantage of this method is that the Fourier coefficients can considerably simplify electronic structure calculations based on envelope function method and plane wave expansion techniques. Regarding those shortcomings such as accuracy depending on the Fourier terms and troublesome performing for the isolated QDs [124], high performance of hardware can come over the accuracy problem and calculation of isolated QDs can 
be achieved by setting adequate periods so long as that QDs can be seen as isolated ones, usually periods are chosen as three times of the base length of QDs [67]. Strain expression for wurtzite QDs have also been formulated in Ref. [116]. Therefore, Green's function model in Fourier domain is selected as the strain calculation model in this work.

\subsubsection{Piezoelectricity calculation}

Calculation of piezoelectric field and piezoelectric potential usually starts from the Maxwell equation $\operatorname{div} \mathbf{D}=0[41,116,130-133]$, where the displacement $\mathbf{D}$ is expressed as,

$$
\mathbf{D}=\varepsilon_{r} \varepsilon_{0} \mathbf{E}+\mathbf{P}
$$

where $\varepsilon_{r}$ is the relative permittivity, $\varepsilon_{0}$ is the permittivity in the vacuum, and the piezoelectric field is related with piezoelectric potential,

$$
V_{p}=-\int E \cdot d l .
$$

For QDs, numerical techniques such as FDM and FFT are adopted to solve piezoelectric field $[41,116]$, while solution of QW case is usually computed analytically. Taking the (0001)-oriented wurtzite QW (assumed as double heterostructure) as an example, piezoelectric field is given by [134-137],

$$
\begin{aligned}
& \mathbf{E}_{z}^{w}=\frac{\mathbf{P}_{b}^{s p}+\mathbf{P}_{b}^{\text {strain }}-\mathbf{P}_{w}^{s p}-\mathbf{P}_{b}^{\text {strain }}}{\varepsilon_{0}\left(\varepsilon_{r}^{w}+\varepsilon_{r}^{b} L^{w} / L^{b}\right)}, \\
& \mathbf{E}_{z}^{b}=-\mathbf{E}_{z}^{w} \frac{L^{w}}{L^{b}}=\frac{\mathbf{P}_{w}^{s p}+\mathbf{P}_{w}^{\text {strain }}-\mathbf{P}_{b}^{s p}-\mathbf{P}_{b}^{\text {strain }}}{\varepsilon_{0}\left(\varepsilon_{r}^{b}+\varepsilon_{r}^{w} L^{b} / L^{w}\right)},
\end{aligned}
$$

where ' $w$ ' and ' $b$ ' denote the well-region and barrier-region, respectively. $L^{w}$ and $L^{b}$ represent the well length and barrier length of QW structure, respectively. $\mathbf{P}^{s p}$ is the spontaneous polarization constant. The strain tensor for QW case is diagonal, 
therefore, only $d_{31}$ and $d_{33}$ are effective in the Eq. (2.39). However, if the structure growths along the [0001] direction, only component $d_{31}$ is important [133]. Consequently, the total strain induced polarization is actually the component along the growth direction, and can be expressed as [133, 134, 138-140],

$$
\begin{aligned}
& \mathbf{P}_{w}^{\text {strain }}=2 e_{13}^{w}\left(C_{11}^{w}+C_{12}^{w}-\frac{2 C_{13}^{w 2}}{C_{33}^{w}}\right) e_{11}^{w}, \\
& \mathbf{P}_{b}^{\text {strain }}=2 e_{13}^{b}\left(C_{11}^{b}+C_{12}^{b}-\frac{2 C_{13}^{b 2}}{C_{33}^{b}}\right) e_{11}^{b} .
\end{aligned}
$$

\subsection{The k·p method}

\subsubsection{Introduction of the $k \cdot p$ method}

\subsubsection{Löwdin perturbation theory}

The $\mathrm{k} \cdot \mathrm{p}$ method itself cannot be formulated without the perturbation theory. The Löwdin Renormalization [141] is one of those perturbative approaches. In the Löwdin perturbation, a reduced set of bands are solved in isolation exactly, while the effects of other bands are added. For example, in the Luttinger-Kohn implementation $[142,143]$, the three valance bands are solved with the effects of the $\mathrm{CB}$ and the other bands added through corrections. These corrections are fitted with experimentally determined values.

\subsubsection{The $k \cdot p$ representation}

Substituting Eq. (2.2) into the time-independent Schrödinger equation Eq. (2.1), one can obtain,

$$
\left[H_{0}+\frac{\hbar}{m_{0}} \mathrm{k} \cdot \mathrm{p}\right] u_{j, \mathbf{k}}(\mathbf{r})=\left[E_{n}(\mathbf{k})-\frac{\hbar^{2} k^{2}}{2 m_{0}}\right] u_{j, \mathbf{k}}(\mathbf{r}),
$$


where,

$$
H_{0}=-\frac{\hbar^{2}}{2 m_{0}} \nabla^{2}+V_{L}(\mathbf{r})
$$

With the Luttinger-Kohn expansion [143],

$$
u_{j, \mathbf{k}}(\mathbf{r})=\sum_{j^{\prime}} \mathbf{c}_{j, j^{\prime}} u_{j^{\prime}, \mathbf{k}_{\mathbf{0}}}(\mathbf{r})
$$

the wavefunction at $\mathbf{k}$ can be expanded in term of $u_{n, \mathbf{k}_{0}}$,

$$
\psi_{j}(\mathbf{r})=\sum_{j^{\prime}} \mathbf{c}_{j, j^{\prime}} e^{i \mathbf{k} \cdot \mathbf{r}} u_{j^{\prime}, \mathbf{k}_{\mathbf{0}}}(\mathbf{r})
$$

Once $E\left(\mathbf{k}_{0}\right)$ and $u_{j, \mathbf{k}_{0}}(\mathbf{r})$ are determined, wavefunction $\psi(\mathbf{r})$ and corresponding energies $E(\mathbf{k})$ at any $\mathbf{k}_{\mathbf{0}}+\Delta \mathbf{k}$ vector in the vicinity of $\mathbf{k}_{\mathbf{0}}$ can be obtained by treating the term $\hbar \Delta \mathrm{k} \cdot \mathrm{p} / m_{0}$ in Eq. (2.56) as a perturbation.

\subsubsection{Development of the $k \cdot p$ method}

The $\mathrm{k} \cdot \mathrm{p}$ method, which follows straightforwardly the Bloch form of wavefunction, is a theoretical tool to calculate energy states and wavefunctions in the vicinity of some important points in the $\mathbf{k}$ space with the aid of perturbation theory [144]. It was originally used by Bardeen [145] and Seitz [146] for various kinds of solids, and later was extended to the much more complicated degenerated band in semiconductors by Shockley [147]. This approach was not defined as a "method" until Dresselhaus $[46,148]$ et al. added the important ingredient of spinorbit interaction and reduced the undetermined constants by adding the symmetry consideration. At the moment, the importance of the $k \cdot p$ method was also established.

Today, the $\mathrm{k} \cdot \mathrm{p}$ method has been developed to calculate the electronic structure of semiconductors widely [40, 46, 59, 70, 72, 107, 142, 143, 147-157]. 
Kane $[144,149,150]$ and Luttinger $[142,143]$ improved the single band theory to the multiband theory via considering the band mixing effect, which was treated with the perturbation theory. As the wide application of quantum heterostructures, strain effect has to be encountered and corresponding effect should be added in the Hamiltonian matrix [107, 153-155]. Strain effect has been incorporated into the Hamiltonian matrix after the pioneer work of Pikus and Bir [151, 152], and Bahder $[107,153,154]$ proposed the eight-band model with strain consideration, later strain gradient term has been included by Braff and Gershoni et al. [158, 159] via making the symmetric replacement to the product of the strain tensor components and the momentum operator. Zhang [155] continued to develop the Hamiltonian with the strain gradient components and added the piezoelectricity effect. Recently, Vukmirović [40] explored the $\bar{C}_{4}$ symmetry of the eight-band $\mathrm{k} \cdot \mathrm{p}$ Hamiltonian for the pyramidal InAs/GaAs QDs and developed a symmetry-based method. This method reduces the volume of Hamiltonian matrix and thus enables a more efficient calculation. Most of the popular models of the $k \cdot p$ method are symmetrical, but the unsymmetrical Hamiltonian was proposed to resist spurious solutions by replacing the traditional SYM operator ordering with the BF operator ordering $[70,71]$. Pokatilov and Fonoberov [72] derived a nonsymmetrical eightband effective-mass Hamiltonian for QDs through employing the BF operator ordering, they also did the comparison between symmetrical and nonsymmetrical models.

Kane model $[144,149,150]$ and Luttinger-Khon model $[142,143]$ are two representative multiband models, and the subsequent $\mathrm{k} \cdot \mathrm{p}$ Hamiltonians are developed based on them, such as Bahder's eight-band Hamiltonian [107, 153, 154] for zinc blende crystals, Chuang's six-band Hamiltonian [106, 160] for wurtzite 
crystals, and the Hamiltonian with the $\mathrm{BF}$ operator ordering $[70,71,73,74]$.

Consequently, detail information about Kane model and Luttinger-Khon model are attached Appendix A and Appendix B, respectively. Other adopted Hamiltonian matrices related to this work will be introduced in the following chapters.

\subsubsection{Numerical schemes to realize the $k \cdot p$ method}

In the exact envelope function theory [100], the electron wavefunction can be written as,

$$
\psi(\mathbf{r})=\sum_{j}^{V} F_{j}(\mathbf{r}) u_{j, 0}(\mathbf{r}),
$$

where $u_{j, 0}(\mathbf{r})$ is the Bloch basis with lattice periodicity, $V$ is the number of bands involved in the model, and $F_{j}(\mathbf{r})$ is the slow-varying envelope functions and is obtained from the position-dependent bulk Hamiltonian matrix $\mathbf{H}(\mathbf{r} ; \mathbf{k})$ in the following form of the Schrödinger equation,

$$
\mathbf{H}(\mathbf{r} ; \mathbf{k}) \mathbf{F}(\mathbf{r})=E(\mathbf{k}) \mathbf{F}(\mathbf{r}) .
$$

Taking QW structure as an example, the graded or abrupt interfaces in QWs have to be considered in the computation by adding a differential operator $\hat{k}_{z}$ in Eq.

$$
\mathbf{H}\left(\mathbf{r} ; k_{\|}, \hat{k}_{z}\right) \mathbf{F}(\mathbf{r})=E\left(k_{\|}, \hat{k}_{z}\right) \mathbf{F}(\mathbf{r})
$$

where $\hat{k}_{z}=-i \partial_{z}$, and the Hamiltonian matrix $\mathbf{H}$ can be expanded into its polynomial for $\hat{k}_{z}$ in the following manner,

$$
\mathbf{H}=H^{(z z)} \hat{k}_{z}^{2}+H^{(z)} \hat{k}_{z}+H^{(0)} .
$$

Consequently, numerical methods such as FEM, FDM, and FTM have to be implemented to solve Eq. (2.62). FEM usually has to meet the intractable 
interpolation problem $[54,55]$, while FDM is much easier to implement but cannot handle the more complicated geometries as FEM. FTM is based on the Fouriertransform technique, naturally avoids the cumbersome differential and integral processes. Since the geometries of low dimensional heterostrucutres are not so complicated, therefore this thesis will only detail the numerical schemes of FDM and FTM to solve Eq. (2.62).

\subsubsection{FDM scheme}

Substituting $\hat{k}_{z}=-i \partial_{z}$ into Eq. (2.63), we have,

$$
\mathbf{H}=-H^{(z z)} \partial_{z}^{2}-i H^{(z)} \partial_{z}+H^{(0)}
$$

And the general FDM scheme for Eq. (2.64) is as follows [106, 161],

$$
\begin{aligned}
\left.H^{(z z)} \partial_{z}^{2} f\right|_{z_{i}} & \left.\rightarrow \partial_{z}\left(H^{(z z)} \partial_{z} f\right)\right|_{z_{i}} \approx \frac{H_{z_{i}+1}^{(z z)}+H_{z_{i}}^{(z z)}}{2(\Delta z)^{2}} f\left(z_{i}+1\right) \\
& +\frac{H_{z_{i}+1}^{(z z)}+2 H_{z_{i}}^{(z z)}+H_{z_{i}-1}^{(z z)}}{2(\Delta z)^{2}} f\left(z_{i}\right)+\frac{H_{z_{i}-1}^{(z z)}+H_{z_{i}}^{(z z)}}{2(\Delta z)^{2}} f\left(z_{i}-1\right) \\
-\left.i H^{(z)} \partial_{z} f\right|_{z_{i}} \rightarrow & \left.\frac{-i}{2}\left[H^{(z)} \partial_{z} f+\partial_{z} H^{(z)} f\right]\right|_{z_{i}} \\
& \approx-i \frac{H_{z_{i}+1}^{(z)}+H_{z_{i}}^{(z)}}{4 \Delta z} f\left(z_{i}+1\right)+i \frac{H_{z_{i}-1}^{(z)}+H_{z_{i}}^{(z)}}{4 \Delta z} f\left(z_{i}-1\right)
\end{aligned}
$$

where $\Delta z$ is mesh step, and $z_{i}$ is the position of the $i$-th mesh point. As the numerical scheme in Eq. (2.65) and Eq. (2.66) applied on Eq. (2.64), one has to create a mesh on the object and deal with the numerical implementation of a differentiation or a integration for a set of coupled equations on the mesh. If number of mesh points is $\mathrm{N}$, the dimension of the banded Hamiltonian matrix is $V N \times V N$. 


\subsubsection{FTM scheme}

It can be seen that solving Eq. (2.62) can be potentially simplified if $z$ dependent Hamiltonian matrix in Eq. (2.63) is expanded in Fourier series as well [60],

$$
\mathbf{H}=\left[\sum_{q} \tilde{H}^{(z z)}(q) e^{i q K z}\right] \hat{k}_{z}^{2}+\left[\sum_{q} \tilde{H}^{(z)}(q) e^{i q K z}\right] \hat{k}_{z}+\sum_{q} \tilde{H}^{(0)}(q),
$$

where $q$ is the order of Fourier series, $\kappa=2 \pi / d$, and $d$ is the period of QW structure. The Fourier series of Hamiltonian matrix $\left(\tilde{H}^{(z z)}, \tilde{H}^{(z)}\right.$, and $\left.\tilde{H}^{(0)}\right)$ are obtained by FFT. No mesh are required if the analytical expression of $\tilde{H}^{(z z)}, \tilde{H}^{(z)}$, and $\tilde{H}^{(0)}$ are provided. By applying $\left\langle f_{2} \mid f_{1}\right\rangle=(1 / L) \int_{L} f_{2}^{*} f_{1} d z$ and the Hermitian operation $\left[\left(Q \hat{k}_{i} f \rightarrow\left[Q \hat{k}_{i} f+\hat{k}_{i} Q f\right] / 2\right.\right.$ and $\left.\left.Q \hat{k}_{i} \hat{k}_{j} f \rightarrow\left(\hat{k}_{i} Q \hat{k}_{j} f+\hat{k}_{j} Q \hat{k}_{i} f\right) / 2\right)\right]$ on Eq. (2.62), the equation for the eigen problem can be transferred into the Fourier domain, and the Hamiltonian matrix in the Fourier domain is [60],

$$
\mathbf{M}=\left(\kappa m+k_{z}\right)\left(\kappa n+k_{z}\right) \tilde{H}^{(z z)}(q)+\left(\frac{\kappa m+\kappa n}{2}+k_{z}\right) \tilde{H}^{(z)}(q)+\tilde{H}^{(0)}(q),
$$

where $q=m-n$. Fourier orders for $\mathrm{QW}$ superlattice at high frequencies have negligible magnitudes and thus can be truncated. If the order of Fourier truncation is up to $N_{t r}$ th order, i.e., $m, n=-N_{t r}, \cdots 0, \cdots N_{t r}$, the Fourier series terms of $\mathbf{H}$ will be reserved up to $2 N_{t r}$ th order, i.e., $q=-2 N_{t r}, \cdots 0, \cdots 2 N_{t r}$. Therefore, the dimension of $\mathbf{M}$ is only determined by $N_{t r}$, i.e., $V\left(2 N_{t r}+1\right) \times V\left(2 N_{t r}+1\right)$. 


\subsubsection{Comparison of FDM and FTM}

FDM and FTM has been compared by Mei [60] from the aspects of numerical scheme, size of Hamiltonian matrix, calculation results, and influencing factors of computational accuracy. The main conclusions are as follows:

1) Adoption of Fourier transform technique in FTM avoids the cumbersome differential process met in FDM.

2) Matrix dimension of FTM is determined by the order of Fourier truncation, while matrix dimension of FDM is determined by the mesh points. The matrix dimension of FTM is far less than that of FDM, as a small order of Fourier truncation can achieve enough accuracy.

3) FTM results and FDM results agree with each other quite well.

4) Accuracy of FTM results may be influenced by the mesh point and order of Fourier truncation, and the influence of the latter factor is more significant. However, accuracy of FDM results is only determined by the mesh points. In both FTM and FDM, more mesh points are demanded for the structures with abrupt interfaces than those with gradded interfaces.

\subsubsection{Spurious solutions in the $k \cdot p$ method}

In contrast to the computationally intensive $a b$ initio and atomistic models, the multiband EMA in the envelope function representation has severed as an important and powerful tool to solve the electronic structures of heterostructures specifically. Unfortunately, the appearance of spurious solutions is one of the main problems of the EMA k·p model, especially for the case with large wave vectors. The so called wing-band (evanescent) solutions [47-50] do not contribute to the effective-mass wavefunctions and thus the elimination of wing-band spurious solutions has been seldom discussed. On the contrary, the oscillatory solutions [51- 
53] are indeed troublesome and the elimination of oscillatory solutions in the $\mathrm{k} \cdot \mathrm{p}$ theory is still a hot topic.

The origin of oscillatory spurious solutions is still controversial and interpreted as multifarious reasons. It has been stated that the perturbative nature and the incomplete set of basis functions of the $\mathrm{k} \cdot \mathrm{p}$ theory cause the nonmonotonic behavior of the $\mathrm{CB}$ with increasing $k$ due to the coupling with VBs $[52,53,75]$. Burt [102] and Foreman [70, 71] ascribed the reason to the adoption of the improper SYM operator ordering, which is not a necessary condition for the multiband Hamiltonian for heterostructures to be Hermitian. Recently, Veprek et al $[73,74]$ demonstrated that necessary fitting of the bulk material parameters to the experimental data for heterostructure calculations leads to the difficulty of satisfying the ellipticity of the differential operator. Lassen et al [61] stated that the envelope functions satisfying the boundary condition would have components outside the first Brillouin zone and, thus, the emergences of the spurious solutions. And even the finite difference scheme may also induce spurious solutions [62].

Although no completely effective way has been put forward to resist the oscillatory solutions, three general methods are proposed to eliminate spurious solutions in some extent. The first is the modification of the Hamiltonian by discarding the coupling between the $\mathrm{CB}$ and the VB [162], by adding a term proportional to $\nabla^{4}$ to the Hamiltonian [53], or by introducing an off-diagonal $k^{2}$ term [162]. This method has not been widely adopted due to the cumbersome implement and the subsequent fixes compromising the initial idea of the effectivemass model. The second is the revision of the improper SYM operator ordering to the BF operator ordering. Spurious solutions in the $\mathrm{CB}$ is further eliminated either by setting the coefficient of $k^{2}$ in the $\mathrm{CB}$ matrix element to zero (i.e. $A_{c}=0$ ) and 
fitting the $\mathrm{CB}$ mass with momentum matrix element $P$ (i.e., optical matrix element $E_{p}$ as $E_{P}=P^{2} 2 m_{0} / \hbar^{2}$ ) [71], or by reducing $E_{p}$ to cater to the ellipticity of the differential operator[73]. This method is verified to be effective to eliminate spurious solutions in the VB [70-74] except for the residual spurious solutions in the CB. The price is that other effective mass parameters also changes simultaneously, and there is still uncertainty about which basis is chosen to determine the literature parameters although $A_{c}$ and $P\left(E_{p}\right)$ can be set to arbitrary values with a change of Bloch basis $[73,77]$. The third is the cut-off method, i.e., discarding the large- $k$ solutions as unphysical. It is very simple and robust for any kind of spurious solutions and thus is widely implemented [47, 49, 61, 75, 76]. Consequently, it should be more effective and powerful to combine the cut-off method with the BF operator ordering to tackle the spurious solution problem in the $\mathrm{k} \cdot \mathrm{p}$ theory, which is main topic of Chapter 5.

\subsection{Long wavelength emission}

Since $1.3 \mu \mathrm{m}$ and $1.55 \mu \mathrm{m}$ are two of the most important wavelengths for optoelectronic interest, most studies aimed to make the light emission wavelength at room temperature of QDs towards the infrared range. The commercial lasers operating at $1.3 \mu \mathrm{m}$ and $1.55 \mu \mathrm{m}$ are presently grown on the InP-substrate, however, the InP-substrate based lasers have several shortcomings, such as high cost [163-166], inferior heat sinking quality [165, 167], and inapplicability for easy fabrication of Bragg stacks required for vertical cavity-surface emitting lasers (VCELs) [164, 168]. The GaAs-substrate based lasers without above metioned drawbacks have become promising candidates for long wave emission [163-168]. In addition, self-assembled InAs/GaAs QDs by the SK method have been 
extensively applied in for laser applications, because of the demand for high-speed, low threshold current density, and temperature-stable lasers with a possible light emission (at room temperature) in the $0.98-1.55 \mu \mathrm{m}$ spectral windows for photonic interest $[65,169]$. A lot of effect has been made to shift the emission wavelength of InAs/GaAs QDs into the infrared region.

Redshift in InAs/GaAs QDs can be circumvented by one of several ways: 1) the grow of InGaAsN on the GaAs-substrate $[163,168,170], 2)$ adopting InP as the confining layer (CL) $[171,172]$, and 3) reducing the compressed strain through adding an In component in the GaAs CL $[64-66,169]$. The large conduction band offset in InGaAsN/GaAs QDs, the possibility of InGaAsN lattice-matched to GaAs-substrate, and cost effectivenss bring about promising devices performances $[163,168]$, whereas, InGaNAs is relatively a new material and incorporation of nitride requires a reduced growth temperature under highly non-equilibrium nucleation conditions to avoid the very poor optical properties [168]. Compared with InAs/GaAs QDs, the smaller initial lattice mismatch in InAs/InP QDs drives InAs/InP QDs to be of great interest for emission in the telecom window (1.4-1.6 $\mu \mathrm{m})$, whereas, the growth of isotropic InAs/InP (100) QDs is much more difficult [173]. Although some progresses on InAs/InP QDs has been achieved either with the $(311)$ B surface $[174,175]$ or with the some optimizations on InAs/InP (100) QDs [175-177], there are still several challenges to realize the real system applications. For instance [173], achieve high gain allowing low threshold current density, obtain high temperature insensitivity for uncooled operation, demonstrate their ability to be modulated at high bit rate with low chirp for optical-fiber transmission, and address the generation of low-jitter pulses in mode-locked lasers. 
The less maturity of InGaAsN/GaAs QDs and InAs/InP QDs leads to a necessary step towards the engineering of method 1) and method 2).

Based on the strain engineering, L. Seravalli et al [64-66] achieved redshift in InAs/GaAs QDs up to $1.5 \mu \mathrm{m}$ by controlling the In composition $x$ in the upper InAs/ $/ n_{x} \mathrm{Ga}_{(1-x)} \mathrm{As}$ confining layer (UCL) and the lower $\operatorname{InAs} / \mathrm{In}_{x} \mathrm{Ga}_{(1-x)} \mathrm{As}$ confining (LCL), and by controlling the thickness $t$ of the LCL. They also investigated the mechanism of redshift with effective mass methods and discussed the thermal quenching of photoluminescence because of the waning of the band discontinuity in their previous studies [64-66]. Nevertheless, some detailed information for this kind of $\operatorname{InAs} / \operatorname{In}_{x} \mathrm{Ga}_{(1-x)} \mathrm{As}$ QDs has not been posted (e. g., no discussion about size fluctuation when $x>0.33$, losing sight of splitting between $\mathrm{HH}$ and $\mathrm{LH}$ bands due to strain action with the "model-solid" theory [178], unclear discussion about influence of strain and band discontinuity on the redshift, and ignorance of the band mixing effect in the calculation). Therefore, a thorough investigation on the electronic structures of this kind of $\operatorname{InAs} / \mathrm{In}_{\mathrm{x}} \mathrm{Ga}_{(1-\mathrm{x})} \mathrm{As}$ QDs is presented using FTM with strain consideration.

\subsection{Interband optical gain spectrum of QWs}

Optical transition together with Fermi-Dirac distribution is shown Figure 25. Gain exists only as condition $E_{g}<\hbar \omega<F_{c}-F_{v}$ is satisfied, where $F_{c}$ and $F_{v}$ are Fermi level in the conduction band and Fermi level in valence band, respectively. In this thesis, only the linear interband gain in QWs is considered, ignoring the exciton effects due to coulomb interaction between electrons and holes, and the screening or many-body effects. 
(a)

(b)

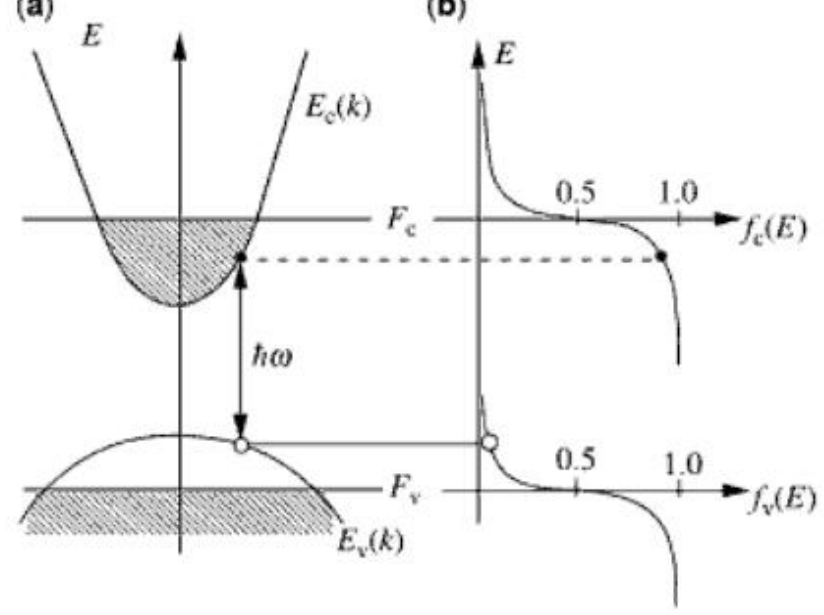

Figure 2-5 Optical transitions. (a) Optical transitions between CB and VBs, and (b) The Fermi-Dirac distributions $f_{c}$ and $f_{v}$. Figure cited after Ref. [22].

\subsubsection{General formulation of the optical gain spectrum}

There are two general ways to calculate the optical gain of materials. One is using the Fermi's golden rule and including the Lorentzian line-shape function [22, 179], which can be replaced by the Sech function to avoid the existence of small absorptions when the photon energy in the calculation is still smaller than the band gap $[85,180,181]$. The other is employing the fundamental relationship between the spontaneous emission and gain spectra [22, 182-184]. Although these two ways are essentially the same, the later one has been adopted more widely for the case with band mixing effect [185-189]. The material gain $(1 / \mathrm{cm})$ is related with spontaneous emission rate with,

$$
g(\hbar \omega)=g_{s p}^{e}(\hbar \omega)\left[1-\exp \left(\frac{\hbar \omega-\Delta F}{k_{B} T}\right)\right],
$$

where $\hat{e}$ denotes the direction of photon polarization, $k_{B}$ is the Boltzmann constant, $T$ is the temperature, $\Delta F=F_{c}-F_{v}$ is the separation of the quasi-Fermi levels. The 
material spontaneous emission rate $g_{s p}^{e}$ of a QW can be calculated with $[185,187$, $189,190]$

$$
\begin{aligned}
g_{s p}^{e}(\hbar \omega) & =C_{0} \sum_{n_{c}, n_{v}} \int \frac{d k_{x} d k_{y}}{4 \pi^{2}}\left|M_{n_{c}, n_{v}}^{e}\left(k_{x}, k_{y}\right)\right|^{2} \\
& \times \frac{f_{n_{c}}^{c}\left(k_{x}, k_{y}\right)\left[1-f_{n_{v}}^{v}\left(k_{x}, k_{y}\right)\right](\hbar \gamma / \pi)}{\left[E_{n_{c}}^{c}\left(k_{x}, k_{y}\right)-E_{n_{v}}^{v}\left(k_{x}, k_{y}\right)-\hbar \omega\right]^{2}+(\hbar \gamma)^{2}},
\end{aligned}
$$

where $C_{0}=q^{2} \pi /\left(n_{r} c \varepsilon_{0} m_{0}^{2} \omega L_{w}\right), c$ is the speed of light in free space, $n_{r}$ is the refractive index of the material, $\varepsilon_{0}$ is the free-space dielectric constant, $m_{0}$ is the electron mass, $q$ is the electron charge, and $\hbar \gamma$ is the half linewidth of the Lorentz function( $\hbar$ is the reduced Planck constant, and $\gamma$ is the relaxation time). $M_{n_{c}, n_{v}}^{e}=\left\langle\psi_{n_{v}}|\hat{e} \cdot \mathbf{p}| \psi_{n_{c}}\right\rangle$ is the momentum matrix element for the transition between $\mathrm{CB}$ state $\psi_{n_{c}}$ and VB state $\psi_{n_{v}}, \mathbf{p}$ is the momentum operator. With the axial approximation, the band structures are assumed to be isotropic in the $k_{x}-k_{y}$ plane, and therefore Eq. (2.70) can be rewritten as,

$$
g_{s p}^{e}(\hbar \omega)=C_{0} \sum_{n_{c}, n_{v}} \int \frac{d k_{t}}{2 \pi}\left|M_{n_{c}, n_{v}}^{e}\left(k_{t}\right)\right|^{2} \times \frac{f_{n_{c}}^{c}\left(k_{t}\right)\left[1-f_{n_{v}}^{v}\left(k_{t}\right)\right](\hbar \gamma / \pi)}{\left[E_{n_{c}}^{c}\left(k_{t}\right)-E_{n_{v}}^{v}\left(k_{t}\right)-\hbar \omega\right]^{2}+(\hbar \gamma)^{2}} .
$$

\subsubsection{Determination of the quasi-Fermi level}

The quasi-Fermi level for the electron can be determined using [22],

$$
N=\sum_{\substack{n=\text { occupied } \\ \text { subands }}} N_{n}=\sum_{\substack{n=\text { occupied } \\ \text { subands }}} \int \rho_{e}^{2 D}(E) f_{c}^{n}(E) d E,
$$

where $N_{n}$ is the electron density in the $n$th conduction subband, and $\rho_{e}^{2 D}(E)$ is the reduced density of states for photons in QWs. With this relationship, the quasiFermi level of $\mathrm{CB}$ can be determined with an iterative method with a given injected electron density $N$. 
Similarly, the hole concentration P can be calculated with [22],

$$
P=N+N_{A}^{-}-N_{D}^{+}=\sum_{\substack{m=o c c u p i e d \\ \text { subands }}} \int \rho_{h}^{2 D}(E)\left[1-f_{v}^{m}(E)\right] d E,
$$

where $N_{A}^{-}$and $N_{D}^{+}$are the ionized electron and hole concentration, respectively. For undoped semiconductors, these two components vanish.

For the parabolic subbands in CB $[182,191]$,

$$
N=\frac{m_{e} k_{B} T}{\pi \hbar^{2} L_{w}} \sum_{n_{c}} \ln \left\{1+\exp \left[-\left(E_{e n_{c}}-F_{c}\right) / k_{B} T\right]\right\}
$$

where $N$ is the electron density, $m_{e}$ is the electron effective mass, and $F_{c}$ is the electron quasi-Fermi level. Summation is over all quantized subbands in $\mathrm{CB}$, and $E_{e n_{c}}$ is the quantized energy level.

For the subband structure is far away from the parabolic, it is more appropriate to find the carrier density by numerically integration over the $k$ space $[182,187,188,191]$

$$
\begin{aligned}
N & =\sum_{n_{c}} \int \frac{1}{4 \pi^{2} L_{w}} f_{c}\left[E_{e n_{c}}\left(k_{x}, k_{y}\right)\right] d k_{x} d k_{y} \\
& \approx \sum_{n_{c}} \int \frac{1}{2 \pi L_{w}} f_{c}\left[E_{e n_{c}}\left(k_{x}, k_{y}\right)\right] d k_{t} \\
P & =\sum_{n_{v}} \int \frac{1}{4 \pi^{2} L_{w}} f_{v}\left[E_{e n_{v}}\left(k_{x}, k_{y}\right)\right] d k_{x} d k_{y} \\
& \approx \sum_{n_{v}} \int \frac{1}{2 \pi L_{w}} f_{v}\left[E_{e n_{v}}\left(k_{x}, k_{y}\right)\right] d k_{t},
\end{aligned}
$$

where $P$ is hole density, $E_{e h_{v}}$ is the hole energy in the valence subbands. $f_{c}$ and $f_{v}$ are the Fermi-Dirac distributions for electrons in CB and holes in VB,

$$
f_{c}=\frac{1}{1+\exp \left[-\left(E_{e n_{c}}-F_{c}\right) / k_{B} T\right]},
$$




$$
f_{v}=\frac{1}{1+\exp \left[-\left(E_{h n_{v}}-F_{v}\right) / k_{B} T\right]} .
$$

$F_{v}$ is the hole quasi-Fermi level.

\subsubsection{Interband transition matrix elements}

According to the plane-wave expansion theory introduced previously, wavefunction of QWs can be written as,

$$
\psi=\sum_{j} F_{j}(\mathbf{k}) u_{j}
$$

where the envelope function is,

$$
F_{j}(\mathbf{k})=e^{i k_{x} x+i k_{y} y} \sum_{n} c_{j}^{n} \phi_{n}(z)
$$

and the plane-wave component is,

$$
\phi_{n}(z)=\frac{1}{\sqrt{L}} \exp \left[i\left(k_{z}+n \frac{2 \pi}{L}\right) z\right] \text {, }
$$

where $L$ is the period of QW superlattice, $F_{j}(\mathbf{k})$ is a slowly varying envelope function along $z$ direction and $u_{j}$ are renormalized $\left|J, m_{j}\right\rangle$ Bloch states at $\boldsymbol{\Gamma}$ point. $\mathbf{c}_{j}^{a, n}$ is the Fourier expansion coefficient.

The transition matrix element between the initial state $a$ and the final state $b$ is,

$$
\begin{aligned}
M_{b a} & =\left\langle\psi_{b}|\hat{e} \cdot \mathbf{p}| \psi_{a}\right\rangle \\
& =\sum_{j, j^{\prime}=1}\left\langle F_{j}(b, \mathbf{k}) u_{j}|\hat{e} \cdot \mathbf{p}| F_{j^{\prime}}(a, \mathbf{k}) u_{j^{\prime}}\right\rangle,
\end{aligned}
$$

where $p=-i \hbar \nabla_{z}$ is the momentum operator and $\hat{e}=\mathbf{i} e_{x}+\mathbf{j} e_{y}+\mathbf{k} e_{z}$ is the photon polarization unit vector. Using the fact that the envelope functions vary relatively slowly over the regions of a unit cell, integral in Eq.(2.82) can be written as [192, 193], 


$$
\begin{aligned}
M_{b a} & =\sum_{j, j^{\prime}=1}\left\langle\sum_{n} c_{j}^{b, n} \phi_{n}(z) u_{j}|\hat{e} \cdot \mathbf{p}| \sum_{n^{\prime}} c_{j^{\prime}}^{a, n^{\prime}} \phi_{n^{\prime}}(z) u_{j^{\prime}}\right\rangle \\
\approx & \sum_{j, j^{\prime}=1}\left\langle\sum_{n} c_{j}^{b, n} \phi_{n}(z) u_{j}|\hat{e} \cdot \mathbf{p}| \sum_{n^{\prime}} c_{j^{\prime}}^{a, n^{\prime}} \phi_{n^{\prime}}(z) u_{j^{\prime}}\right\rangle \times\left\langle u_{j} \mid u_{j^{\prime}}\right\rangle \\
& +\sum_{j, j^{\prime}=1}\left\langle\sum_{n} c_{j}^{b, n} \phi_{n}(z) \mid \sum_{n^{\prime}} c_{j^{\prime}}^{a, n^{\prime}} \phi_{n^{\prime}}(z)\right\rangle\left\langle u_{j}|\hat{e} \cdot \mathbf{p}| u_{j^{\prime}}\right\rangle, \\
= & \sum_{j=1}\left\langle\sum_{n} c_{j}^{b, n} \phi_{n}(z)|\hat{e} \cdot \mathbf{p}| \sum_{n^{\prime}} c_{j}^{a, n^{\prime}} \phi_{n^{\prime}}(z)\right\rangle\left(j=j^{\prime}\right) \\
& +\sum_{j} \sum_{j^{\prime}} \sum_{n}\left[\left(c_{j}^{b, n}\right)^{*} \cdot c_{j^{\prime}}^{a, n}\right]\left\langle u_{j}|\hat{e} \cdot \mathbf{p}| u_{j^{\prime}}\right\rangle
\end{aligned}
$$

the first term on the right of Eq. (2.83) describes the intraband transition, and the second term represents the interband transition. Consequently, the interband transition matrix elements in Eq.(2.70) and Eq. (2.71) can be expressed as,

$$
M_{n_{c}, n_{v}}^{e}=\sum_{j} \sum_{j^{\prime}} \sum_{n}\left[\left(c_{j}^{n_{v}, n}\right)^{*} \cdot c_{j^{\prime}}^{n_{c}, n}\right]\left\langle u_{j}|\hat{e} \cdot \mathbf{p}| u_{j^{\prime}}\right\rangle
$$

\subsection{Summary}

This chapter provides an overview of reported research work on the modeling electronic structures and optical properties of low dimensional heterostrucutres, which is summarized as follows:

1) The theoretical fundamentals of modeling electronic structures and optical gain of low dimensional heterostructures.

2) Computation models for strain and piezoelectricity. This chapter gives a sufficient review about strain computation models and shows that the one based on the Green's function and solved in the Fourier domain is the most proper method for this study.

3) Development and research challenges as using the $\mathrm{k} \cdot \mathrm{p}$ method to model electronic structures of low dimensional herostrucutres. This chapter briefs the development of the $\mathrm{k} \cdot \mathrm{p}$ theory itself, then move onto numerical methods 
to implement the $\mathrm{k} \cdot \mathrm{p}$ method, and finally reviews the biggest challenge (spurious solutions) to use the $\mathrm{k} \cdot \mathrm{p}$ method. The reviews shows that FTM has exceeds the previous numerical methods, from the perspectives of convenience, possibility to realize meshless programming, and possibility to overcome spurious solutions, as well as capability to simplify the optical gain computation.

4) Achievements and challenges about electronic structures and optical properties of low dimensional heterostructures. This chapter supplements the information in Chapter 1 and provides a review about strain effects, long wavelength emission, and optical gain computation, paving the way for the studies in the next chapters. 


\section{CHAPTER 3}

\section{FORMULATION AND COMPUTATION}

\section{OF FTM FOR QDS}

To implement the $\mathrm{k} \cdot \mathrm{p}$ method for the QD calculation, formulation of FTM is developed using the Fourier transform technique and the orthogonality of exponential function. QD shape effect can be incorporated into the computation either numerically or analytically. The meshless FTM is formulated by adopting the analytical Fourier transform of QD shape function directly. This chapter illustrates that FTM is applicable to isolated QDs, and discusses the critical factors that affect the computation accuracy as well.

\subsection{Formulation of FTM for QDs}

The envelope function of a QD superlattice can be expanded in plane waves along $x, y$, and $z$,

$$
\mathbf{F}(\mathbf{r})=\frac{1}{\sqrt{d_{x} d_{y} d_{z}}} e^{i k_{x} x+i k_{y} y+i k_{z} z} \sum_{n_{x}} \sum_{n_{y}} \sum_{n_{z}} c_{n_{x} n_{y} n_{z}} e^{i\left(n_{x} \kappa_{x} x+n_{y} \kappa_{y} y+n_{z} \kappa_{z} z\right)}
$$

where $d_{x}, d_{y}$, and $d_{z}$ are periods along $\mathrm{x}, \mathrm{y}$, and $\mathrm{z}$ directions, $\kappa_{\alpha}=\frac{2 \pi}{d_{\alpha}},-\frac{\kappa_{\alpha}}{2}<k_{\alpha}<\frac{\kappa_{\alpha}}{2}$ for $\alpha=x, y, z$, and $\mathbf{c}_{n_{x} n_{y} n_{z}}=\left[c_{1} c_{2} \cdots c_{V}\right]_{n_{x} n_{y} n_{z}}^{T}$. If there are $N$ superlattice periods, the Born-Von Karman cyclic boundary condition [99] gives,

$$
k_{\alpha}=\frac{2 \pi j}{N d_{\alpha}}, 0 \leq j \leq N, \alpha=x, y, z
$$


Eq. (2.61) can be completely handled in Fourier domain with Fourier series of $\mathrm{H}(\mathrm{r} ; \mathrm{k})$ [60]. This approach takes advantage of orthogonality of complex componential functions to evade the cumbersome integral calculation and presents the equation for eigen problem as,

$$
\left[\mathbf{M}_{u m, v n}\right]\left[\mathbf{c}_{v n}\right]=E\left[\mathbf{c}_{v n}\right] \text {, }
$$

where the Hamiltonian matrix in Fourier domain, $\mathbf{M}_{u m, v n}$, is expressed with Fourier series $\tilde{H}^{(\alpha \beta)}, \tilde{H}^{(\alpha)}$ and $\tilde{H}^{(0)}$ of the multiband k p p Hermitian matrix of the real space Hamiltonian,

$$
\begin{aligned}
\mathbf{M}_{s t}= & \frac{1}{2} \sum_{\alpha, \beta}^{x, y, z} \tilde{H}_{j j^{\prime}, q_{i}=m_{i}-n_{i}}^{(\alpha \beta)}\left[\left(k_{\beta}+n_{\beta} \kappa_{\beta}\right)\left(k_{\alpha}+n_{\alpha} \kappa_{\alpha}\right)+\left(k_{\alpha}+n_{\alpha} \kappa_{\alpha}\right)\left(k_{\beta}+n_{\beta} \kappa_{\beta}\right)\right] \\
& +\frac{1}{2} \sum_{\alpha}^{x, y, z} \tilde{H}_{j j^{\prime}, q_{i}=m_{i}-n_{i}}^{(\alpha)}\left[2 k_{\alpha}+\left(m_{\alpha}+n_{\alpha}\right) \kappa_{\alpha}\right] \\
& +\tilde{H}_{j j^{\prime}, q_{i}=m_{i}-n_{i}}^{(0)}
\end{aligned}
$$

where $i=x, y, z$. The derivation is presented in Appendix C. The dimension of the Fourier domain Hamiltonian $\mathrm{M}$ is only determined by the order of the Fourier frequencies of $\mathrm{H}$. An arbitrary heterostructure may result in an infinite dimension of $\mathrm{M}$, but practically, the Fourier spectrum has negligible magnitudes at high frequencies and thus can be truncated. If the terms up to $N^{\text {th }}$ order in Fourier series are retained in the wavefunction, i.e., $n_{\alpha}, m_{\alpha}=-N_{\alpha}, \ldots 0, \ldots, N_{\alpha}$, the Fourier series terms of $\mathrm{H}$ will be reserved up to $2 N_{\alpha}^{\text {th }}$ order, i.e., $q_{\alpha}=m_{\alpha}-n_{\alpha}=-2 N_{\alpha}, \cdots 0, \cdots 2 N_{\alpha}$. Therefore, the dimension of $\mathrm{M}$ is only determined by the truncation frequencies $N_{\alpha}$, i.e. $V\left(2 N_{x}+1\right)\left(2 N_{y}+1\right)\left(2 N_{z}+1\right)$ $\times V\left(2 N_{x}+1\right)\left(2 N_{y}+1\right)\left(2 N_{z}+1\right)$. The subscripts for Eq. (3.4) is schemed as,

$$
s=j N_{x} N_{y} N_{z}+\left(m_{z}+N_{z}\right) N_{x} N_{y}+\left(m_{y}+N_{y}\right) N_{x}+\left(m_{x}+N_{x}+1\right),
$$




$$
t=j^{\prime} N_{x} N_{y} N_{z}+\left(n_{z}+N_{z}\right) N_{x} N_{y}+\left(n_{y}+N_{y}\right) N_{x}+\left(n_{x}+N_{x}+1\right)
$$

where $j, j^{\prime}=1,2, \ldots, V$ and $V$ is the number of bands involved in the calculation.

\subsection{Implementation of FTM for QDs}

\subsubsection{QD shape function}

For the SK epitaxial growth of QDs, a thin wetting layer is usually formed before the spontaneous coherent island formation. It does provide a weak QW confining effect, however its influence is very weak and usually neglected in the computation. For a better illustration of the implementation of FTM for QDs, wetting layer effect on electronic structures of QDs is ignored here and will be discussed in the next chapter. Consequently, a simplified geometric calculation model, which is shown in Figure 3-1 with a pyramidal QD as an example, is adopted in this chapter. The geometric shape function within a periodic box is defined to distinguish the regions of the dot and the barrier materials,

$$
\Lambda(r)=\left\{\begin{array}{l}
1, \text { well island } \\
0, \text { barrier region }
\end{array} .\right.
$$

The corresponding geometric shape function in Fourier domain can be derived by,

$$
\tilde{\Lambda}(\xi)=\int_{\Omega} \Lambda(\mathbf{r}) e^{-i \xi \cdot \mathbf{r}} d V
$$

where $\xi$ is the Cartesian coordinate in Fourier domain and $\Omega$ denotes the periodic box, and the integration is carried out over the periodic box. Assuming a material parameter $Q_{d}$ in the dot region and $Q_{b}$ in the barrier region, the distribution function of the material parameter is expressed as, 


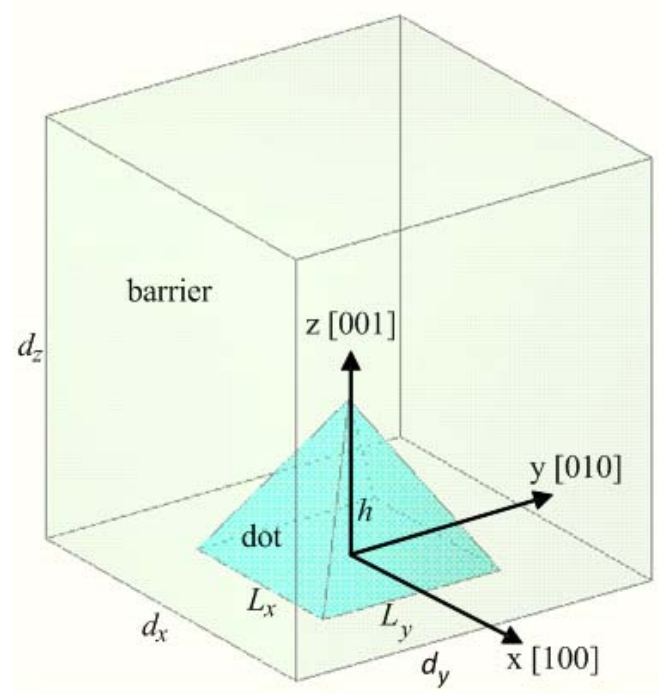

Figure 3-1 Geometric model of a pyramidal QD, where the dot is buried in the periodic box, $d_{x}, d_{y}$, and $d_{z}$ are the dimensions of the periodic box along $x, y$, and $z$ directions, respectively.

$$
Q(\mathbf{r})=Q_{d} \Lambda(\mathbf{r})+Q_{b}[\Omega(\mathbf{r})-\Lambda(\mathbf{r})]
$$

where $\Omega(\mathbf{r})$ is a periodic cuboid box with dimensions as $d_{1}, d_{2}$, and $d_{3}$ along $x, y$, and $z$ directions, respectively. Therefore the corresponding Fourier transform of the distribution function of the material parameter is,

$\tilde{Q}(\xi)=\left(Q_{d}-Q_{b}\right) \tilde{\Lambda}(\xi)+Q_{b} \tilde{\Omega}(\xi)$,

where $\tilde{\Omega}(\xi)$ is the Fourier transform of the periodic box. Analytical Fouriertransform expressions of several QD shapes are cited after Andreev et al $[67,116]$, where the singular values of those expressions were not provided. These singular values are attached in Appendix D.

\subsubsection{Constructing the Hamiltonian matrix in the Fourier domain}

The kinetic piece of an eight-band $\mathrm{k} \cdot \mathrm{p}$ Hamiltonian matrix $\mathbf{H}_{k}[107,153$, 154] (see Appendix F) is adopted to formulate the Hamiltonian matrix in Fourier domain $\mathbf{M}_{\mathbf{k}}$. A “convenient length", $l_{0}=1.9519 \AA$, renders $\hbar^{2} / 2 m_{0} l_{0}^{2}=1 \mathrm{eV}$ and thus 
the wave vectors $k_{x}, k_{y}$, and $k_{z}$ are expressed in the unit of $1 / l_{0}$ accordingly.

Therefore, with the differential operator $\hat{k}_{\alpha}$ usually utilized in heterostructures, the kinetic piece of the Hamiltonian is written as,

$$
\mathbf{H}_{k}=\left[\begin{array}{cccccccc}
A & 0 & V^{*} & 0 & \sqrt{3} V & -\sqrt{2} U & -U & \sqrt{2} V^{*} \\
0 & A & -\sqrt{2} U & -\sqrt{3} V^{*} & 0 & -V & \sqrt{2} V & U \\
V & -\sqrt{2} U & -P+Q & -S^{*} & R & 0 & \sqrt{3 / 2} S & -\sqrt{2} Q \\
0 & -\sqrt{3} V & -S & -P-Q & 0 & R & -\sqrt{2} R & \sqrt{1 / 2} S \\
\sqrt{3} V^{*} & 0 & R^{*} & 0 & -P-Q & S^{*} & \sqrt{1 / 2} S^{*} & \sqrt{2} R^{*} \\
-\sqrt{2} U & -V^{*} & 0 & R^{*} & S & -P+Q & \sqrt{2} Q & \sqrt{3 / 2} S^{*} \\
-U & \sqrt{2} V^{*} & \sqrt{3 / 2} S^{*} & -\sqrt{2} R^{*} & \sqrt{1 / 2} S & \sqrt{2} Q & Z & 0 \\
\sqrt{2} V & U & -\sqrt{2} Q & \sqrt{1 / 2} S^{*} & \sqrt{2} R & \sqrt{3 / 2} S & 0 & Z
\end{array}\right],
$$

where,

$$
\begin{aligned}
& A=E_{c}+G_{c}\left(\hat{k}_{x}^{2}+\hat{k}_{y}^{2}+\hat{k}_{z}^{2}\right) \\
& P=-E_{v}+\gamma_{1}\left(\hat{k}_{x}^{2}+\hat{k}_{y}^{2}+\hat{k}_{z}^{2}\right) \\
& Q=\gamma_{2}\left(\hat{k}_{x}^{2}+\hat{k}_{y}^{2}-2 \hat{k}_{z}^{2}\right) \\
& Z=E_{v}-\Delta-\gamma_{1}\left(\hat{k}_{x}^{2}+\hat{k}_{y}^{2}+\hat{k}_{z}^{2}\right) \\
& U=\frac{1}{\sqrt{3}} G_{c v} \hat{k}_{z} \\
& V=\frac{1}{\sqrt{6}} G_{c v}\left(\hat{k}_{x}-i \hat{k}_{y}\right) \\
& R=-\sqrt{3}\left[\gamma_{2}\left(\hat{k}_{x}^{2}-\hat{k}_{y}^{2}\right)-2 i \hat{k}_{3} \hat{k}_{x} \hat{k}_{y}\right] \\
& S=2 \sqrt{3} \gamma_{3} \hat{k}_{z}\left(\hat{k}_{x}-i \hat{k}_{y}\right)
\end{aligned}
$$

where $E_{c}$ and $E_{v}$ are the unstrained conduction band-edge and valence band-edge respectively, and $\Delta$ is the spin-orbit splitting. Parameter $G_{c}, G_{c v}$ are expressed as, 


$$
\begin{aligned}
& G_{c}=\left[\frac{m_{0}}{m_{c}}-\frac{E_{p}}{3}\left(\frac{2}{E_{g}}+\frac{1}{E_{g}+\Delta}\right)\right], \\
& G_{c v}=\sqrt{E_{p}}
\end{aligned}
$$

where $m_{c}$ is the effective electron mass, $m_{0}$ is usually chosen as the unit of $m_{c}, E_{p}$ is the optical matrix parameter, and $E_{g}=E_{c}-E_{v}$ is the energy gap. Modified Luttinger parameters $\gamma_{1}, \gamma_{2}$, and $\gamma_{3}$ are defined as [194],

$$
\begin{aligned}
& \gamma_{1}=\gamma_{1}^{L}-\frac{E_{p}}{3 E_{g}} \\
& \gamma_{2}=\gamma_{2}^{L}-\frac{E_{p}}{6 E_{g}}, \\
& \gamma_{3}=\gamma_{3}^{L}-\frac{E_{p}}{6 E_{g}}
\end{aligned}
$$

where $\gamma_{1}^{L}, \gamma_{2}^{L}$ and $\gamma_{3}^{L}$ are the corresponding usual Luttinger parameters.

With Eq. (3.8), the shape effect of QDs enters the above material parameters naturally, and thus all the nonzero components $A, P, Q, Z, U, V, R$, and $S$ in kinetic Hamiltonian matrix $\mathbf{H}_{\mathbf{k}}$ include the shape effect of QDs. According to the form of these nonzero components, all of them can be expressed as the following format,

$$
\begin{aligned}
T & =\sum_{\alpha, \beta}^{x, y, z} T^{(\alpha \beta)} \hat{k}_{\alpha} \hat{k}_{\beta}+\sum_{\alpha}^{x, y, z} T^{(\alpha)} \hat{k}_{\alpha}+T^{(0)} \\
& =\sum_{\alpha, \beta}^{x, y, z} C_{T}^{(\alpha \beta)} Q(r) \hat{k}_{\alpha} \hat{k}_{\beta}+\sum_{\alpha}^{x, y, z} C_{T}^{(\alpha)}(T) Q(r) \hat{k}_{\alpha}+C_{T}^{(0)}(T) Q(r)
\end{aligned},
$$

where $C_{T}^{(l)}$ is constant coefficient and $l$ denotes “ $\alpha \beta$ ”, “ $\alpha$ ”, and “ 0 ”. Therefore, with Eq. (3.9), the Fourier transform of $T^{(l)}$ can be obtained as,

$$
\tilde{T}^{(l)}=C_{T}^{(l)}\left[\left(Q_{d}-Q_{b}\right) \tilde{\Lambda}(\xi)+Q_{b} \tilde{\Omega}(\xi)\right] .
$$


Comparing Eq. (3.15) and Eq. (C.2), components of $H^{(l)}$ in Eq. (C.2) can be represented with $T^{(l)}$ accordingly. Therefore, Fourier series $\tilde{H}^{(l)}$ in Eq. (3.4) can be expressed with $\tilde{T}^{(l)}$,

$$
\tilde{H}_{k}^{(l)}(\xi)=\left[\left(Q_{d}-Q_{b}\right) \tilde{\Lambda}(\xi)+Q_{b} \tilde{\Omega}(\xi)\right] H H_{k},
$$

where,

$$
\cdot H H_{k}=\left[\begin{array}{cccccccc}
C_{A}^{(l)} & 0 & C_{V}^{(l) *} & 0 & \sqrt{3} C_{V}^{(l)} & -\sqrt{2} C_{U}^{(l)} & -C_{U}^{(l)} & \sqrt{2} C_{V}^{(l) *} \\
0 & C_{A}^{(l)} & -\sqrt{2} C_{U}^{(l)} & -\sqrt{3} C_{V}^{(l) *} & 0 & -C_{V}^{(l)} & \sqrt{2} C_{V}^{(l)} & C_{U}^{(l)} \\
C_{V}^{(l)} & -\sqrt{2} C_{U}^{(l)} & -C_{P}^{(l)}+C_{Q}^{(l)} & -C_{S}^{(l)^{*}} & C_{R}^{(l)} & 0 & \sqrt{\frac{3}{2}} C_{S}^{(l)} & -\sqrt{2} C_{Q}^{(l)} \\
0 & -\sqrt{3} C_{V}^{(l)} & -C_{S}^{(l)} & -C_{P}^{(l)}-C_{Q}^{(l)} & 0 & C_{R}^{(l)} & -\sqrt{2} C_{R}^{(l)} & \sqrt{\frac{1}{2}} C_{S}^{(l)} \\
\sqrt{3} C_{V}^{(l) *} & 0 & C_{R}^{(l) *} & 0 & -C_{P}^{(l)}-C_{Q}^{(l)} & C_{S}^{(l) *} & \sqrt{\frac{1}{2}} C_{S}^{(l) *} & \sqrt{2} C_{R}^{(l) *} \\
-\sqrt{2} C_{U}^{(l)} & -C_{V}^{(l) *} & 0 & C_{R}^{(l) *} & C_{S}^{(l)} & -C_{P}^{(l)}+C_{Q}^{(l)} & \sqrt{2} C_{Q}^{(l)} & \sqrt{\frac{3}{2}} C_{S}^{(l)^{*}} \\
-C_{U}^{(l)} & \sqrt{2} C_{V}^{(l) *} & \sqrt{\frac{3}{2}} C_{S}^{(l) *} & -\sqrt{2} C_{R}^{(l) *} & \sqrt{\frac{1}{2}} C_{S}^{(l)} & \sqrt{2} C_{Q}^{(l)} & C_{Z}^{(l)} & 0 \\
\sqrt{2} C_{V}^{(l)} & C_{U}^{(l)} & -\sqrt{2} C_{Q}^{(l)} & \sqrt{\frac{1}{2}} C_{S}^{(l) *} & \sqrt{2} C_{R}^{(l)} & \sqrt{\frac{3}{2}} C_{S}^{(l)} & 0 & C_{Z}^{(l)}
\end{array}\right]
$$

Finally, the Hamiltonian matrix $\mathbf{M}_{\mathbf{k}}$ can be constructed with Eq. (3.4) and Eq.(3.16) conveniently. Up to now, the QD geometric shape effect has been combined into FTM and the meshless FTM has been formulated. The meshless FTM not only inherits predominance of traditional FTM but also avoids the use of mesh to achieve higher accuracy, benefiting from the given analytical Fourier-transform expressions of QD geometric functions.

Regarding the case with strain consideration, the strain part of the eightband $\mathrm{k} \cdot \mathrm{p}$ Hamiltonian matrix $\mathbf{H}_{\mathrm{s}}$ in Ref. $[107,153,154]$ is used (see Appendix F) and this $\mathbf{H}_{\mathrm{s}}$ is treated in the same way as that of the kinetic Hamiltonian matrix $\mathbf{H}_{\mathrm{k}}$. Therefore, the strain piece of the Hamiltonian is written as, 


$$
\mathbf{H}_{s}=\left[\begin{array}{cccccccc}
a & 0 & -v^{*} & 0 & -\sqrt{3} v & \sqrt{2} u & u & -\sqrt{2} v^{*} \\
0 & a & \sqrt{2} u & \sqrt{3} v^{*} & 0 & v & -\sqrt{2} v & -u \\
-v & \sqrt{2} u & -p+q & -s^{*} & r & 0 & \sqrt{3 / 2} s & -\sqrt{2} q \\
0 & \sqrt{3} v & -s & -p-q & 0 & r & -\sqrt{2} r & \sqrt{1 / 2} s \\
-\sqrt{3} v^{*} & 0 & r^{*} & 0 & -p-q & s^{*} & \sqrt{1 / 2} s^{*} & \sqrt{2} r^{*} \\
\sqrt{2} u & v^{*} & 0 & r^{*} & s & -p+q & \sqrt{2} q & \sqrt{3 / 2} s^{*} \\
u & -\sqrt{2} v^{*} & \sqrt{3 / 2} s^{*} & -\sqrt{2} r^{*} & \sqrt{1 / 2} s & \sqrt{2} q & -a_{v} e & 0 \\
-\sqrt{2} v & -u & -\sqrt{2} q & \sqrt{1 / 2} s^{*} & \sqrt{2} r & \sqrt{3 / 2} s & 0 & -a_{v} e
\end{array}\right],
$$

where,

$$
\begin{aligned}
a & =a_{c} e_{h} \\
p & =a_{v} e_{h} \\
q & =b e_{b} \\
u & =\sqrt{\frac{1}{3}} G_{c v} \sum_{j} e_{3 j} \hat{k}_{j}, \\
r & =\frac{\sqrt{3}}{2} b\left(e_{11}-e_{22}\right)-i d e_{12} \\
v & =\sqrt{\frac{E_{P}}{6}} \sum_{j}\left(e_{1 j}-i e_{2 j}\right) \hat{k}_{j} \\
s & =-d\left(e_{13}-i e_{23}\right)
\end{aligned}
$$

where $e_{h}=e_{11}+e_{22}+e_{33}$ is the hydrostatic strain component; $e_{b}=e_{z z}-\left(e_{x x}+e_{y y}\right) / 2$ is the biaxial strain component, $a_{c}$ is the deformation potential of $\mathrm{CB}, a_{v}$ is the hydrostatic deformation potential of $\mathrm{VB}$, both $b$ and $d$ are the shear deformation potentials.

Following the form of nonzero elements in $\mathbf{H}_{\mathrm{k}}$, all nonzero elements $\mathbf{H}_{\mathrm{s}}$ can be rewritten as,

$$
\begin{aligned}
t & =\sum_{\alpha, \beta}^{x, y, z} t^{(\alpha \beta)} \hat{k}_{\alpha} \hat{k}_{\beta}+\sum_{\alpha}^{x, y, z} t^{(\alpha)} \hat{k}_{\alpha}+t^{(0)} \\
& =\sum_{\alpha, \beta}^{x, y, z} C_{t}^{(\alpha \beta)} Q(r) e^{(\alpha \beta)}(r) \hat{k}_{\alpha} \hat{k}_{\beta}+\sum_{\alpha}^{x, y, z} C_{t}^{(\alpha)} Q(r) e^{(\alpha)}(r) \hat{k}_{\alpha}+C_{t}^{(0)} Q(r) e^{(0)}(r)
\end{aligned}
$$


where $C_{t}^{(l)}$ is constant coefficient and $e^{(l)}$ is the corresponding strain component. Therefore, with Eq. (3.9), the Fourier transform of $t^{(l)}$ can be obtained as,

$$
\tilde{t}^{(l)}=C_{t}^{(l)}\left[\left(Q_{d}-Q_{b}\right) \sum_{\xi^{\prime}} \tilde{\Lambda}\left(\xi-\xi^{\prime}\right) \tilde{e}_{i j}^{(l)}\left(\xi^{\prime}\right)+Q_{b} \sum_{\xi^{\prime}} \tilde{\Omega}\left(\xi-\xi^{\prime}\right) \tilde{e}_{i j}^{(l)}\left(\xi^{\prime}\right)\right] \text {. }
$$

Similar to the kinetic Hamiltonian matrix, the strain Hamiltonian matrix in the Fourier domain can also be expressed as,

$$
\tilde{H}_{s}^{(l)}(\xi)=C_{t}^{(l)}\left[\left(Q_{d}-Q_{b}\right) \sum_{\xi^{\prime}} \tilde{\Lambda}\left(\xi-\xi^{\prime}\right) \tilde{e}_{i j}^{(l)}\left(\xi^{\prime}\right)+Q_{b} \sum_{\xi^{\prime}} \tilde{\Omega}\left(\xi-\xi^{\prime}\right) \tilde{e}_{i j}^{(l)}\left(\xi^{\prime}\right)\right] H H_{s},
$$

where,

$$
H H_{s}=\left[\begin{array}{cccccccc}
c_{a}^{(l)} & 0 & -c_{v}^{(l) *} & 0 & -\sqrt{3} c_{v}^{(l)} & \sqrt{2} c_{u}^{(l)} & c_{u}^{(l)} & -\sqrt{2} c_{v}^{(l) *} \\
0 & c_{a}^{(l)} & \sqrt{2} c_{u}^{(l)} & \sqrt{3} c_{v}^{(l) *} & 0 & c_{v}^{(l)} & -\sqrt{2} c_{v}^{(l)} & -c_{u}^{(l)} \\
-c_{v}^{(l)} & \sqrt{2} c_{u}^{(l)} & -c_{p}^{(l)}+c_{q}^{(l)} & -c_{s}^{(l) *} & c_{r}^{(l)} & 0 & \sqrt{\frac{3}{2}} c_{s}^{(l)} & -\sqrt{2} c_{q}^{(l)} \\
0 & \sqrt{3} c_{v}^{(l)} & -c_{s}^{(l)} & -c_{p}^{(l)}-c_{q}^{(l)} & 0 & c_{r}^{(l)} & -\sqrt{2} c_{r}^{(l)} & \sqrt{\frac{1}{2}} c_{s}^{(l)} \\
-\sqrt{3} c_{v}^{(l) *} & 0 & c_{r}^{(l) *} & 0 & -c_{p}^{(l)}-c_{q}^{(l)} & c_{s}^{(l) *} & \sqrt{\frac{1}{2}} c_{s}^{(l) *} & \sqrt{2} c_{r}^{(l) *} \\
\sqrt{2} c_{u}^{(l)} & c_{v}^{(l) *} & 0 & c_{r}^{(l) *} & c_{s}^{(l)} & -c_{p}^{(l)}+c_{q}^{(l)} & \sqrt{2} c_{q}^{(l)} & \sqrt{\frac{3}{2}} c_{s}^{(l)^{*}} \\
c_{u}^{(l)} & -\sqrt{2} c_{v}^{(l) *} & \sqrt{\frac{3}{2}} c_{s}^{(l) *} & -\sqrt{2} c_{r}^{(l)^{*}} & \sqrt{\frac{1}{2}} c_{s}^{(l)} & \sqrt{2} c_{q}^{(l)} & c_{z}^{(l)} & 0 \\
-\sqrt{2} c_{v}^{(l)} & -c_{u}^{(l)} & -\sqrt{2} c_{q}^{(l)} & \sqrt{\frac{1}{2}} c_{s}^{(l) *} & \sqrt{2} c_{r}^{(l)} & \sqrt{\frac{3}{2}} c_{s}^{(l)} & 0 & c_{z}^{(l)}
\end{array}\right] .
$$

Ultimately, the Hamiltonian matrix $\mathbf{M}_{\mathbf{s}}$ comprises $\tilde{H}_{s}^{(l)}$, and the whole Hamiltonian matrix with strain consideration is $\mathbf{M}=\mathbf{M}_{\mathbf{k}}+\mathbf{M}_{\mathbf{s}}$. Alternatively, $\tilde{t}^{(l)}$ may be obtained by performing numerical Fourier transform of $t^{(l)}$ with Eq.(3.9) and Eq. (3.20)-(3.21). In the following computation, the latter approach is adopted to avoid the challenging and time-consuming convolution in Eq. (3.22). 


\subsection{FTM computations for isolated QDs}

\subsubsection{Control of the computation accuracy}

\subsubsection{Strain computation}

For the strain calculation, a Fourier transform method developed by Andreev et al [67] is utilized. This method can calculate the strain distribution of QDs simply and conveniently. The drawback lies at the case of isolated QDs: the periodic length needs to be selected carefully so that the influence from other periods can be negligible. Therefore, it is necessary to determine the most proper periodic length for the computation of isolated QDs. All of the material parameters in this chapter are shown in Table 3-1.

Table 3-1 Material parameters of InAs and GaAs. These parameters are cited after Ref.[109].

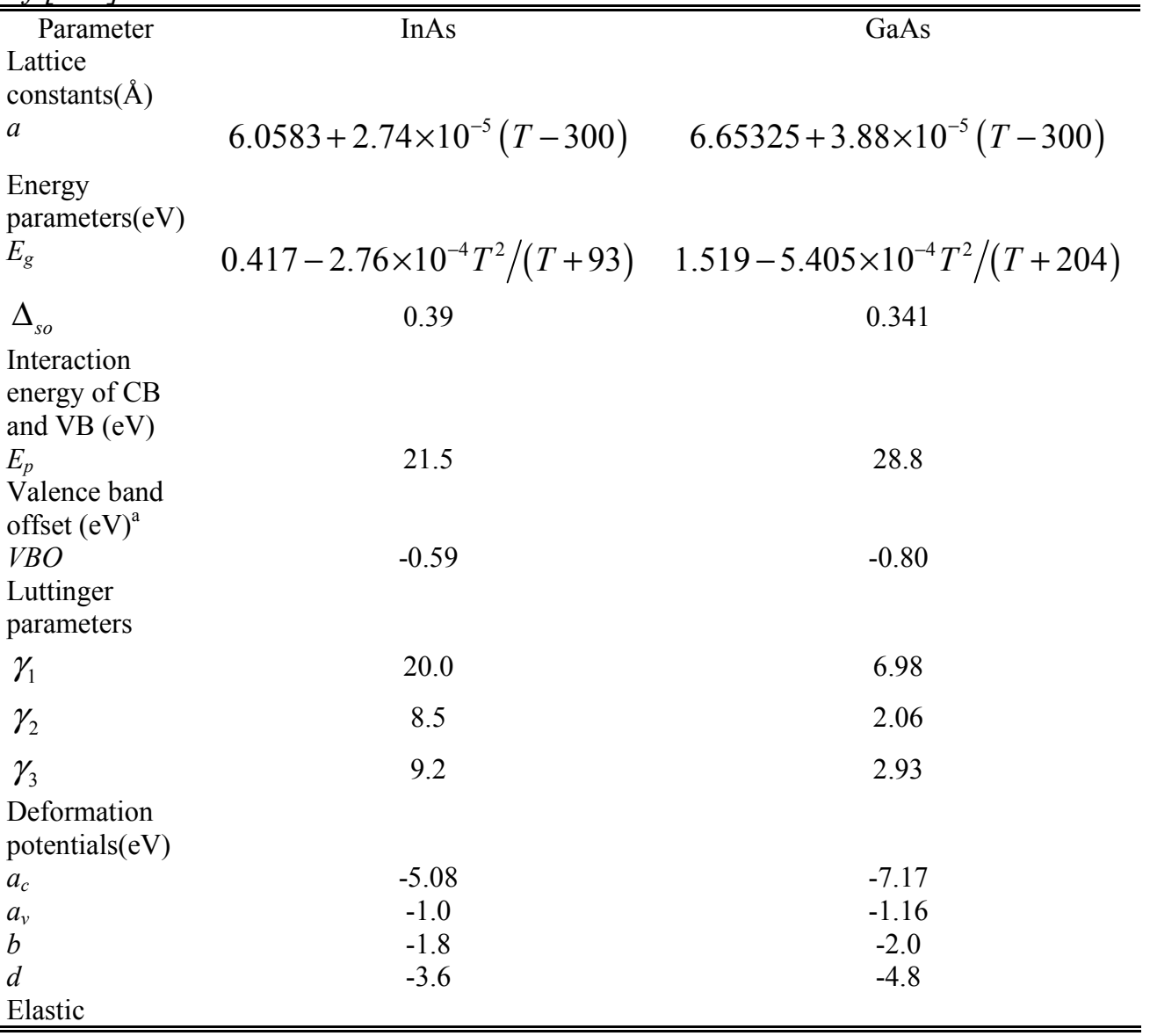


stiffness

constants

(Gpa)

\begin{tabular}{llc}
$C_{11}$ & 83.29 & 122.1 \\
$C_{12}$ & 45.26 & 56.6 \\
$C_{44}$ & 39.59 & 60.0 \\
\hline \hline
\end{tabular}

a. The origin of energy states refers to the maximum of $\mathrm{VB}$ of InSb.

An integration method [124] formulates an analytical strain calculation formula based on the inclusion theory of continuum mechanism and the Green's function conception, which are also the basic theory of the Fourier transform strain calculation method. The main difference between these two methods is the material parameter. The Fourier transform method employs stiffness constants $C_{11}$, $C_{12}$ and $C_{44}$, while the integration method uses Poisson's ratio $v$ and Young's modulus E. Actually, both Poisson's ratio $v$ and Young's modulus $E$ can be derived from the stiffness constants. Hence, the difference caused by the input material parameters in these two methods should be very small and thus negligible. Consequently, it is reasonable to cite the results reported in Ref. [124] as the criterion to determine the proper periodic length directly. The strain distributions ( $e_{11}, e_{33}$, and $e_{b}$ components) of structures B (InAs/GaAs pyramidal QD) and D (InAs/GaAs half-truncated pyramidal QD) are numerically cited from Ref. [124] as the criterion.

The geometrical dimension of QD examples B and D and the employed periodic length are tabulated in Table 3-2, the label associated with periodic length is $a$ - $d$. The strain components $e_{11}, e_{33}$, and $e_{b}$ of structure B and structure D are plotted along the $\mathrm{z}([001])$ direction in Figure 3-2 and Figure 3-3, respectively. $R$ is the cited criterion, $a-d$ denote the strain distribution calculated using the Fourier transform method with different periodic lengths. As the periodic length varies from $\left[2 L_{x}, 2 L_{x}, 2 \mathrm{~h}(1-f)\right]$ to $\left[3 L_{x}, 3 L_{x}, 3 \mathrm{~h}(1-f)\right]$, the calculated strain components 
approach to the criterion lines gradually. However, the calculated strain results are further closer to the criterion lines as the periodic length along the $z$ direction $\left(d_{3}\right)$ continuatively increases. Except for the interfaces of dot and barrier, the calculated strain results superpose on the criterion lines completely as $d_{3}$ varies from $6 \mathrm{~h}(1-f)$ to $3 L_{x}$. Therefore, both $\left[2 L_{x}, 2 L_{x}, 6 \mathrm{~h}(1-f)\right]$ and $\left[3 L_{x}, 3 L_{x}, 3 L_{x}\right]$ can be chosen as the periodic length. From the view of numerical computation, $\left[3 L_{x}, 3 L_{x}, 3 L_{x}\right]$ is the most proper choice and is employed in the work of Andreev et al [67] to calculate strain in isolated QDs.

Table 3-2 Dimension and periodic length of InAs/GaAs QDs. $f$ is the shape truncation factor. $f$ are selected as $f=0$ for $Q D$ structure $B$, and $f=0.5$ for the $Q D$ structure $D$ (The dimension of the upper surface is $L_{x} f \times L_{y} f$ ).

\begin{tabular}{ccccccc}
\hline \hline InAs/GaAs & \multicolumn{3}{c}{ Dimension $(\AA)$} & \multicolumn{3}{c}{ Periodic length $(\AA)$} \\
& $L_{x}$ & $L_{y}$ & $h$ & $d_{1}$ & $d_{2}$ & $d_{3}$ \\
$\mathrm{a}$ & 155 & 155 & 55 & $2 L_{x}$ & $2 L_{y}$ & $2 \mathrm{~h}$ \\
$\mathrm{~b}$ & 155 & 155 & 55 & $3 L_{x}$ & $3 L_{y}$ & $3 \mathrm{~h}$ \\
$\mathrm{c}$ & 155 & 155 & 55 & $3 L_{x}$ & $3 L_{y}$ & $6 \mathrm{~h}$ \\
$\mathrm{~d}$ & 155 & 155 & 55 & $3 L_{x}$ & $3 L_{y}$ & $3 L_{x}$ \\
\hline \hline
\end{tabular}

One may argue that the above conclusion may not go through as the wetting layer is added in the calculation. Therefore, the strain components $e_{11}, e_{33}$, and $e_{b}$ of structure $\mathrm{B}$ and structure $\mathrm{D}$ with a $5 \AA$ wetting layer included in the calculation are plotted along the $z([001])$ direction in Figure 3-4 and Figure 3-5, respectively. The two solid lines in Figure 3-4 and Figure 3-5 denote the interface of wetting layer and the dot region. The influence of periodical length on strain distribution does not change and $\left[3 L_{x}, 3 L_{x}, 3 L_{x}\right]$ is still the most proper choice, even the wetting layer is included in the computation. The detail variation of strain distribution due to the wetting layer will be presented in Chapter 4.

In Figure 3-2, there are distinguished sharp features in the strain components calculated using the integration method. In contrast, no sharp features appear in Figure 3-3. These sharp features are regarded as the divergence problem 
due to the distinguished change of material parameters. The Fourier transform method can restrain these sharp features but not the integration method. Stier and Grundmann et al [39] also mentioned that FEM results of the continuum mechanical model have the divergence problem while the FDM results of the continuum mechanical model do not have this problem.

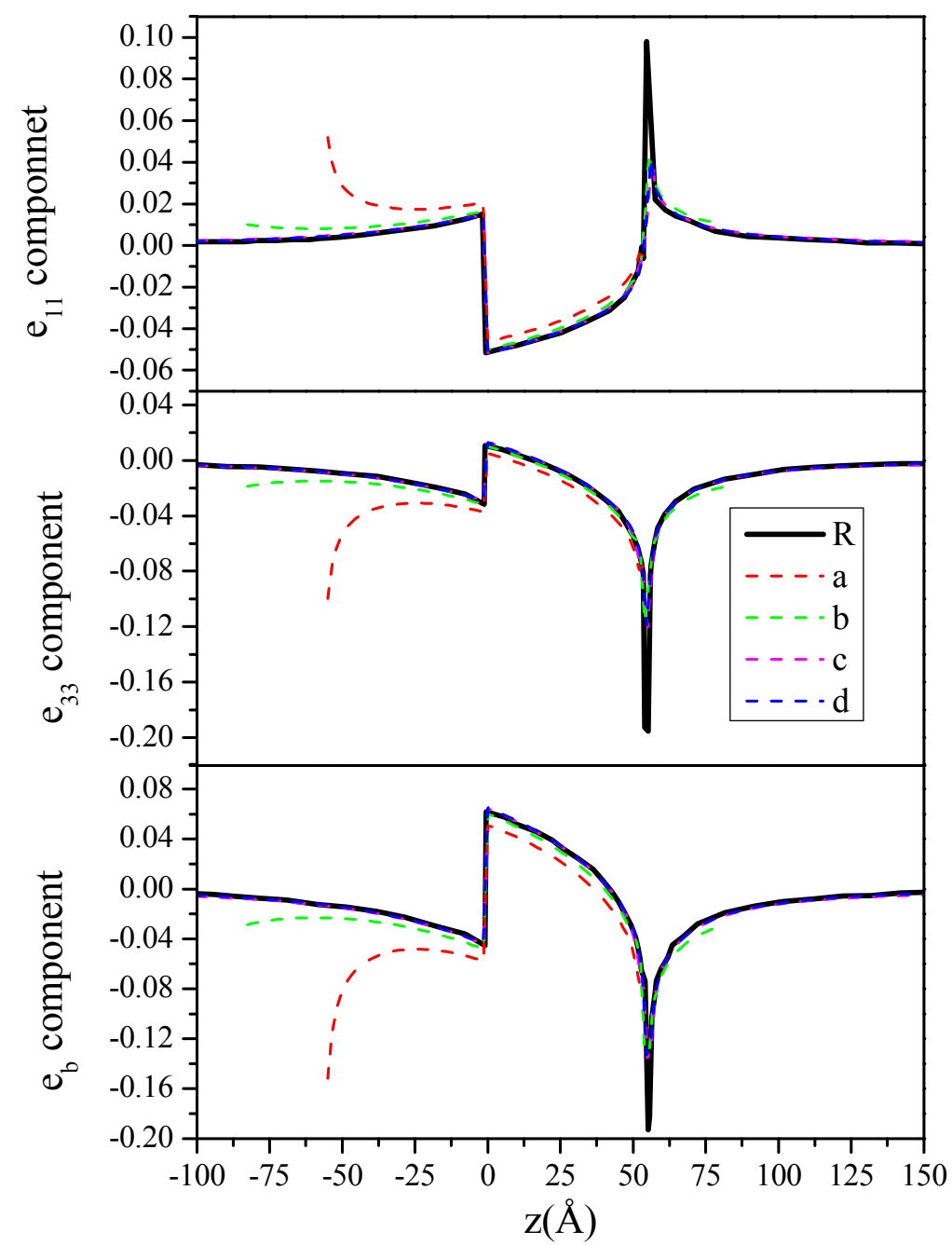

Figure 3-2 Influence of the periodic length on the strain distribution of the isolated InAs/GaAs pyramidal QDs $(f=0) . R$ is the criterion cited after Ref. [124], a-d denote the strain distribution calculated using the Fourier transform method with different periodic lengths. 


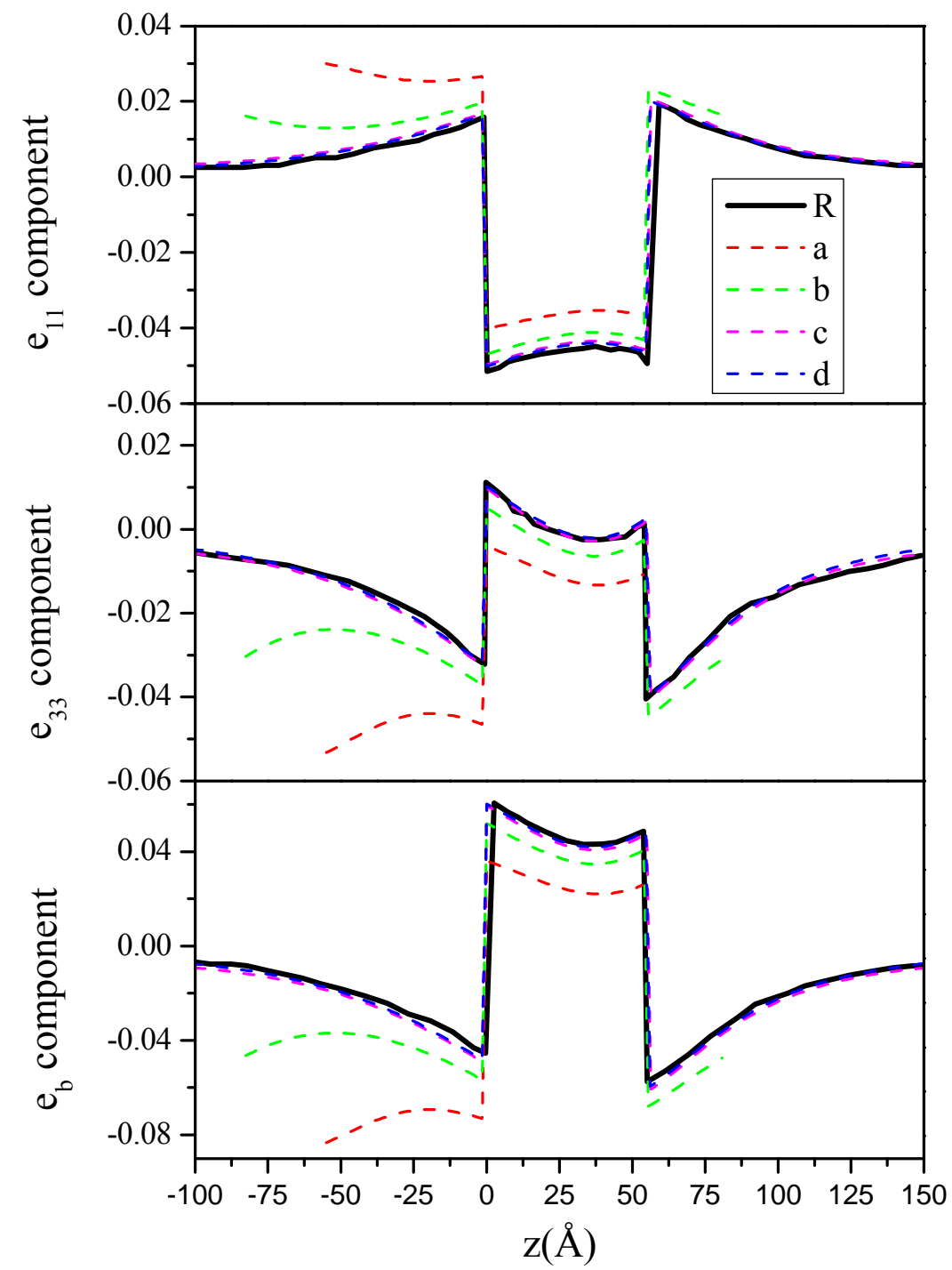

Figure 3-3 Influence of the periodic length on the strain distribution of the isolated InAs/GaAs half-truncated pyramidal QDs $(f=0.5)$. $R$ is the criterion cited after Ref. [124], a-d denote the strain distribution calculated using the Fourier transform method with different periodic lengths. The dark line represents the interface between the dot region and the wetting layer region. 


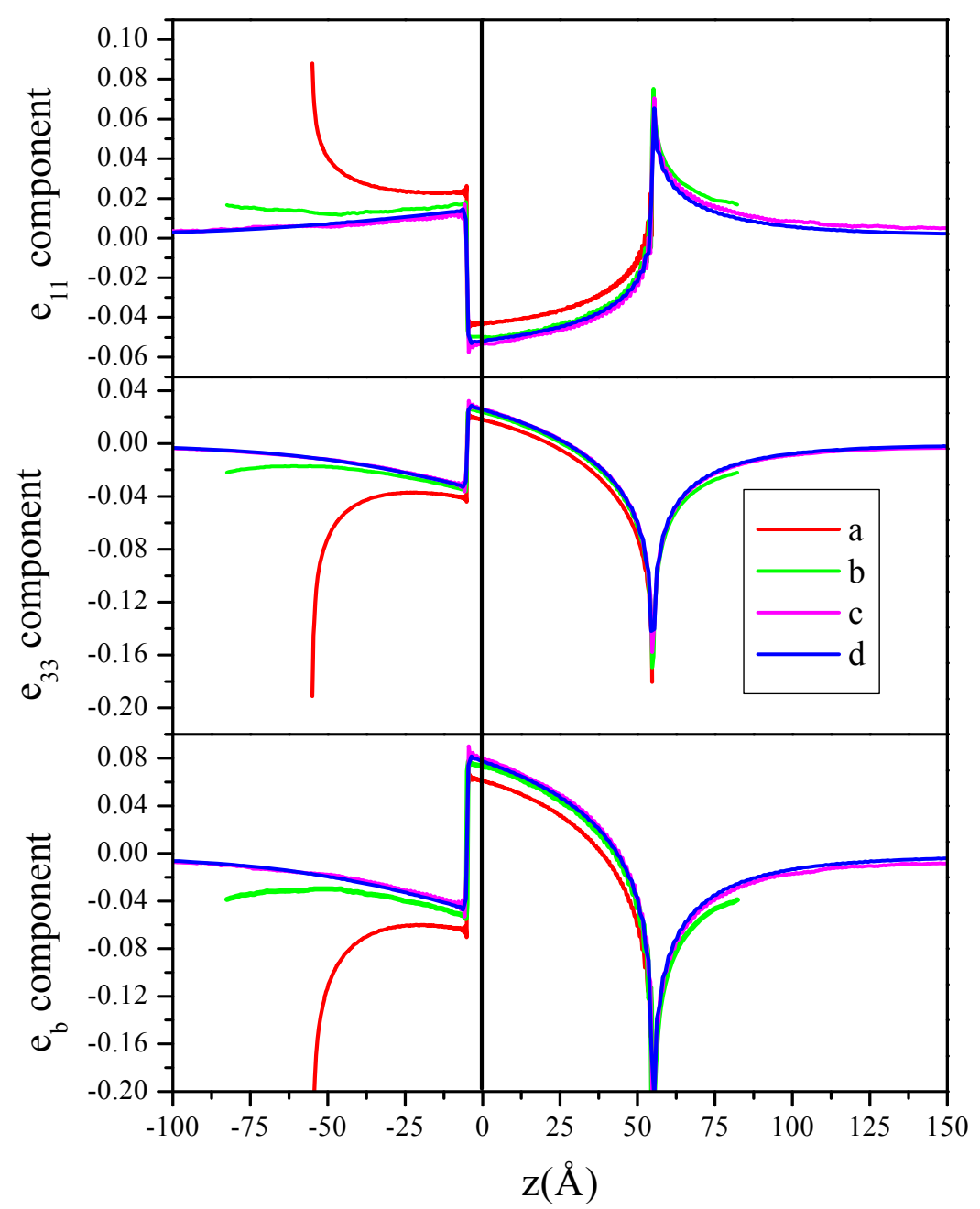

Figure 3-4 Influence of the periodical length on strain distribution of the isolated InAs/GaAs pyramidal $Q D s(f=0)$ as wetting layer is included. a-d denote the strain distribution calculated using the Fourier transform method with different periodical lengths. The solid line represents the interface of the dot region and the wetting layer region. 


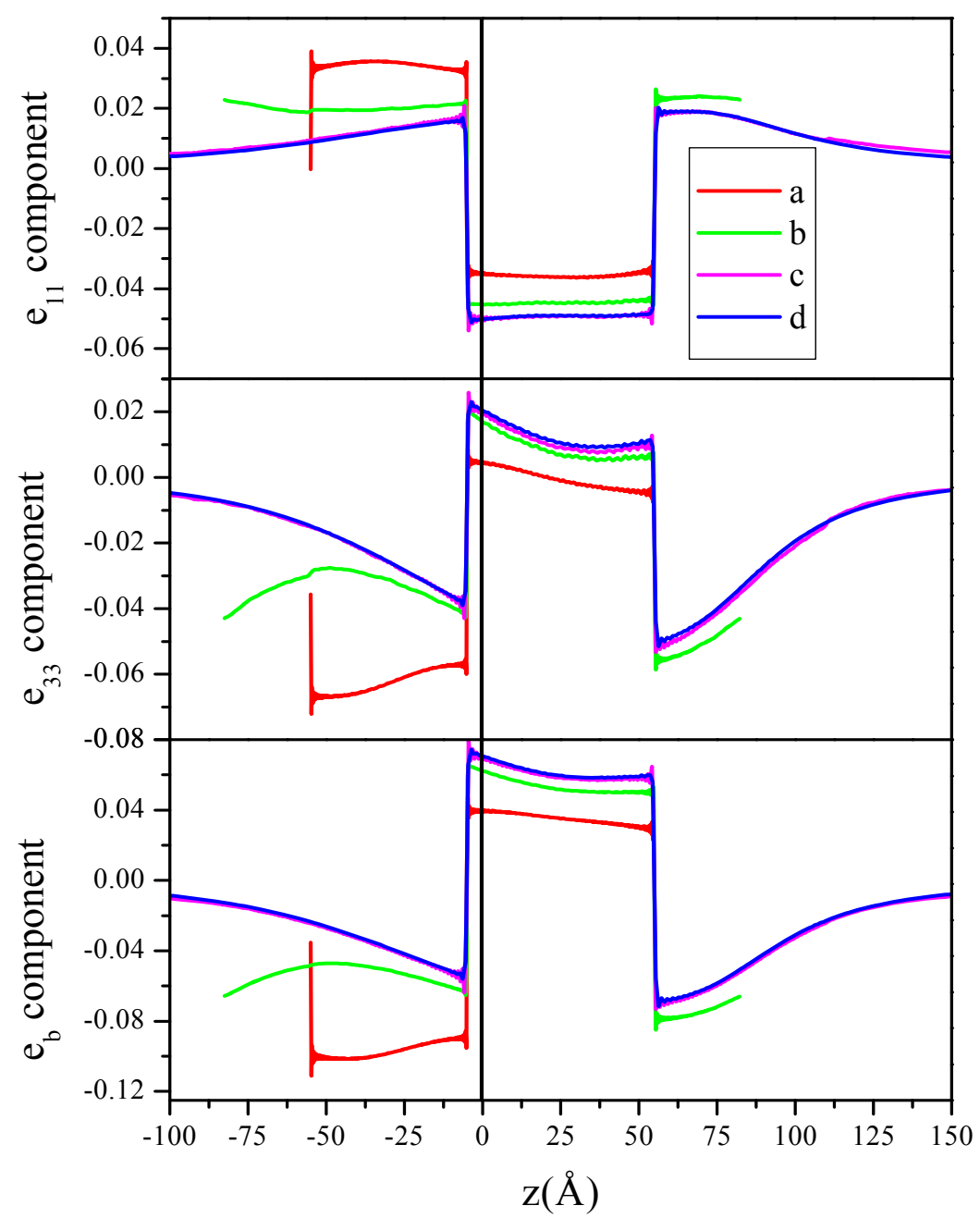

Figure 3-5 Influence of the periodical length on strain distribution of the isolated InAs/GaAs half-trucnated pyramidal $Q D s(f=0.5)$ as wetting layer is included. a-d denote the strain distribution calculated using the Fourier transform method with different periodical lengths. The solid line represents the interface of the dot region and the wetting layer region.

\subsubsection{FTM computation}

Application of FTM on electronic structures of QDs is quite similar as that of QWs, the comparison between FTM with other numerical methods such as FDM has been detailed by Mei [60]. This thesis will not repeat this part with the QD computation. 
FTM is applicable to the QD superlattice structures perfectly except for the fact that a truncation error is caused by truncating the infinite Fourier series as in Eq. (3.1). For an isolated QD structure where periodicity does not exist, finite periodic lengths have to be adopted in order to apply the cyclic boundary condition and, thus, a crosstalk error is imposed. Such a situation in the calculation using FTM is quite similar to the situation in the calculation with the single-band EMA [34]. Consequently, it is necessary to minimize the truncation error and the crosstalk error through choosing the proper order of Fourier truncation and periodic length.

As mentioned previously, the truncation of higher orders of Fourier series is a very useful technique to reduce the dimension of Hamiltonian matrix, but truncation error is unavoidable. Thus, a proper order of Fourier truncation is essential to mediate the conflict between a smaller matrix dimension and higher calculation accuracy. Therefore, the reported pyramidal QD structure [39] with $L_{x}=136 \AA, L_{y}=136 \AA$ and $h=60 \AA$ is used to investigate the effects of the Fourier truncation and the periodic length $d$ synchronously. Together with $N_{t r}$ varying from 1 to 7 , a series of $\mathrm{d} / L_{x}(1,1.25,1.5,1.75,2,2.25,2.5,2.75,3,3.5,4,4.5$ and 5$)$, is adopted to find the proper order of Fourier truncation $N_{t r}$ and the periodic length $d$. Figure 3-6 illustrates that the ground-state energies of electrons, E1, and groundstate energies of HHs, $\mathrm{HH} 1$, vary as a function of $L / L_{x}$ under different $N_{t r}$. E1 is affected by both the order of Fourier truncation and the periodic length more easily, and HH1 is mainly influenced by the order of Fourier truncation as $L / L_{x}$ exceeds 2 . In contrast, the ground-state energies of LHs are insensitive to either the order of Fourier truncation or the periodic length and, thus, are omitted. Consequently, E1 is selected to find the most proper Fourier truncation order. 
As $L / L_{x}$ increases from 1 to 2 , the dominant error is the crosstalk error, which is caused by the tunneling of electrons from the surrounding periods. In reverse, the dominant error is the truncation error under long periodic length $\left(L / L_{x}=4,5\right)$. Therefore, increasing the periodic lengths reduces the crosstalk error, but increases the truncation error due to raised high-order Fourier harmonics. Accordingly, a medial periodic length of $L / L_{x}=3$, which is same as that in the strain computation, is chosen finally throughout this study. Interestingly, the popular periodic length is also selected as $L / L_{x}=3$ in Ref. [67] and Ref. [34].

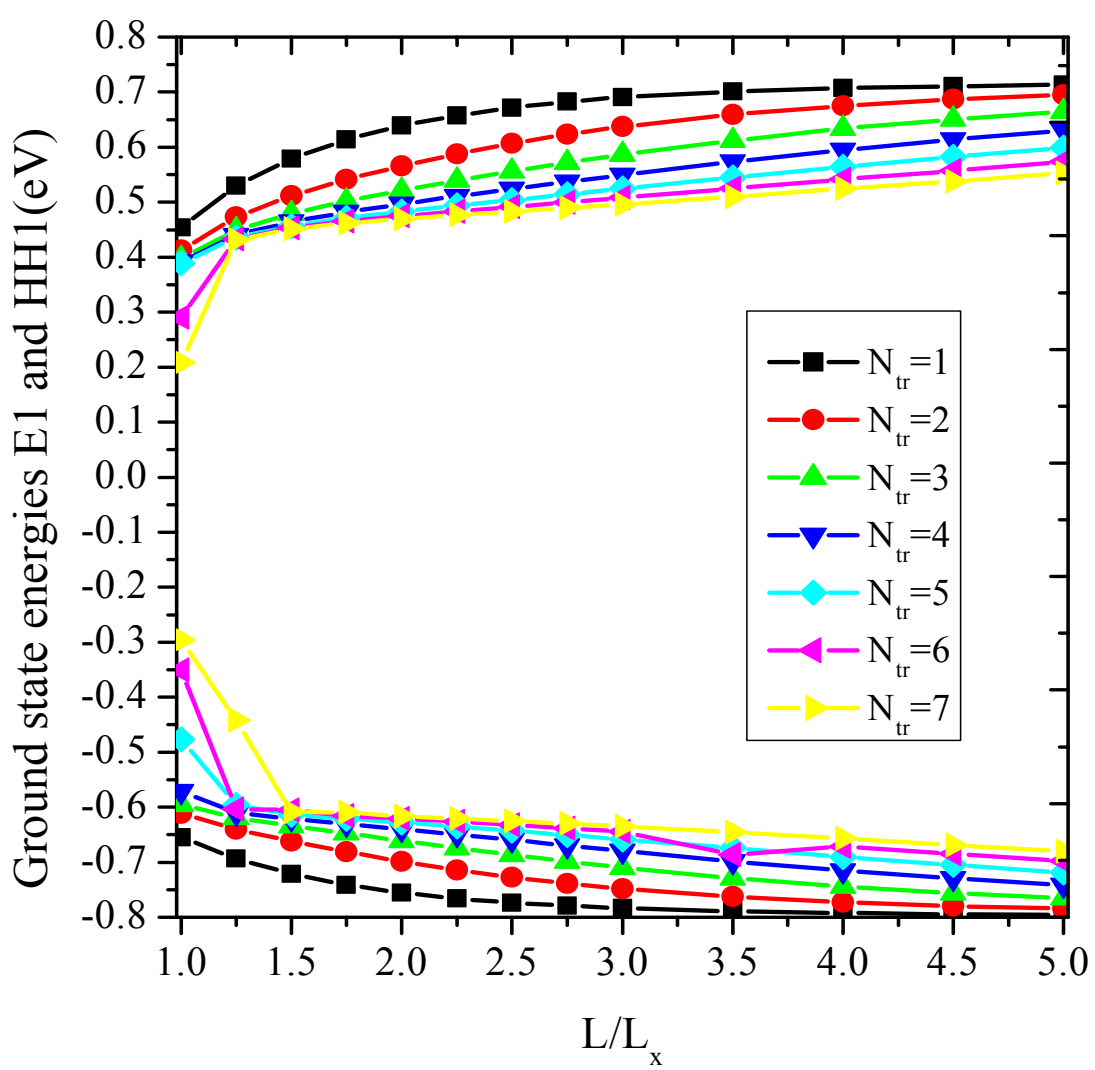

Figure 3-6 Ground-state energies E1 and HH1 of the pyramidal $Q D$ with $L_{x}=136$ $A, L_{y}=136 \AA$ and $h=60 \AA$ vary as a function of $L / L x$ under different $N_{t r}$. 


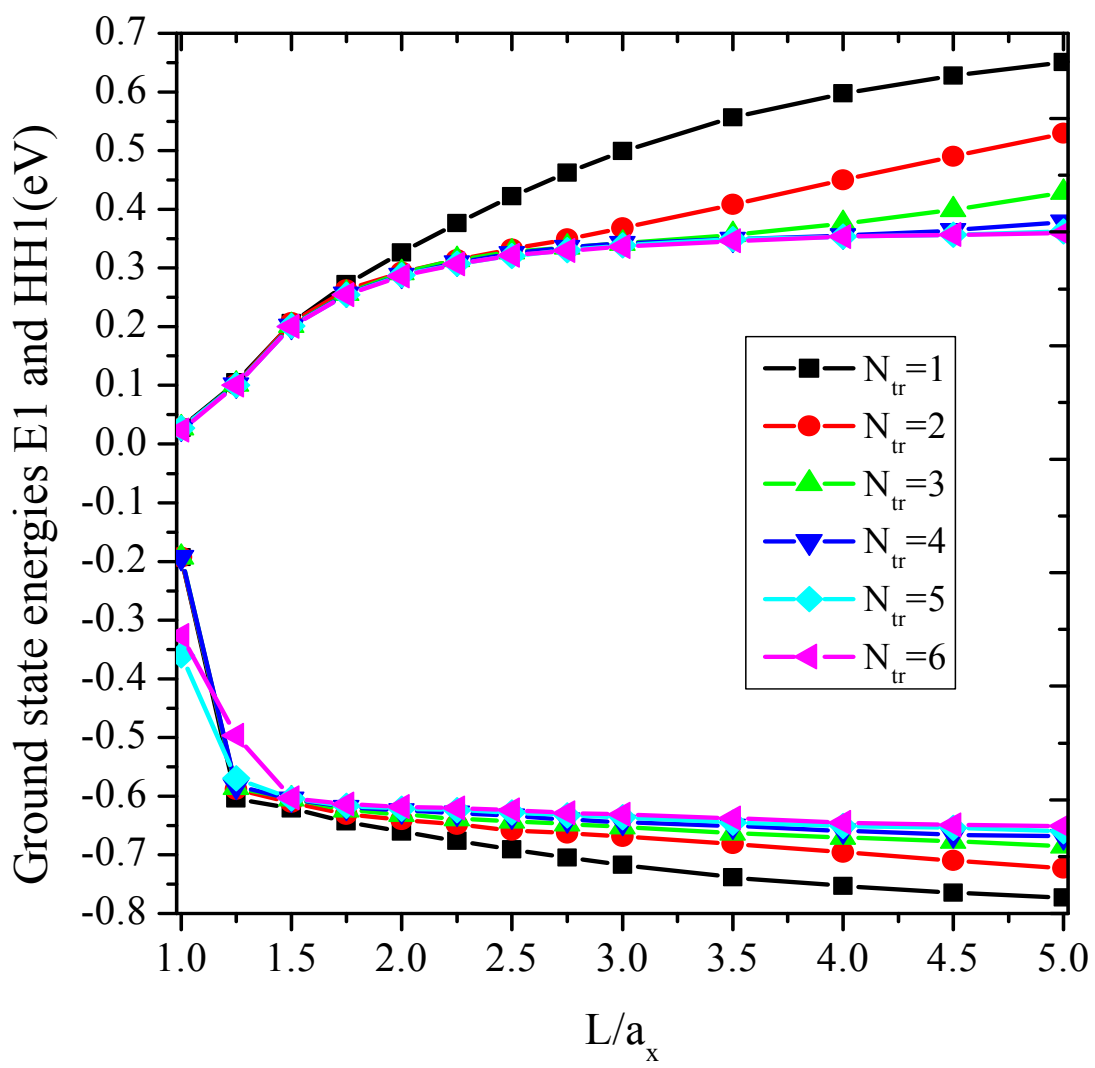

Figure 3-7 Ground-state energies E1 and HH1 of the cuboidal QD with $a_{x}=a_{y}=$ $a_{z}=136$ A vary as a function of $L / a_{x}$ under different $N_{t r}$.

As stated in the previous work [60], structures with smooth geometries possess a narrow-span Fourier spectrum. Thus, a lower order of Fourier truncation is required to achieve enough accuracy of the computation results. This can also be found in Figure 3-7, which presents the ground-state energies E1 and HH1 of a cuboidal QD with $\mathrm{a}_{x}=a_{y}=a_{z}=136 \AA$ varying as a function of $L / a_{x}$ under different $N_{t r}$. The dashed line denotes the trend of energy states when $N_{t r}$ is sufficiently large. Variations due to a change of the periodic length and the order of Fourier truncation in Figure 3-7 are almost the same as the pyramidal case shown in Figure 3-6, except that energy state E1 can almost superpose on the dash line at $L / a_{x}=3$ 
and $N_{t r}=6$. This indicates that there is no convergence problem. Regarding the small convergence problem in Figure 3-6 even at $N_{t r}=7$, it is the tradeoff between the demand of a higher order of Fourier truncation for sharper structures and the limit of computer capacity. Fortunately, the error due to this tradeoff is still in the acceptable range.

In brief, $L / L_{x}=3$ (or $L / \mathrm{a}_{x}=3$ ) is applicable for different shapes of QDs. However, a higher order of Fourier truncation $N_{t r}$ is required for the QDs with a sharp feature. The criterion to determine the two critical parameters should be chosen based on both accuracy and memory requirement.

\subsubsection{Further reduction of the memory consumption}

Some accessorial techniques have been used to reduce the memory requirement. For instance, the size of the Hamiltonian matrix was reduced by exploiting the $C_{4 v}$ symmetry of the QD structure [34] and the $\bar{C}_{4}$ symmetry of the eight-band $\mathrm{k} \cdot \mathrm{p}$ Hamiltonian [40]. However, it is complicated to realize the symmetry consideration. Truncation of higher Fourier frequencies is the most effective way to reduce the memory demand, and the memory requirement can be further lowered by eliminating the very small components in the Hamiltonian matrix in Eq. (3.4). Of course, the precondition of these two operations is that computation accuracy should be guaranteed.

\subsection{Summary}

FTM with a SYM operator ordering have been presented as an approach to solving the $3 \mathrm{D}$ multiband $\mathrm{k} \cdot \mathrm{p}$ eigen problems. The Fourier domain Hamiltonian expressed in a neat form is derived from the position-dependent Hamiltonian in the real space. This method allows the incorporation of analytical shape functions in 
the Fourier domain and can make a meshless $\mathrm{k} \cdot \mathrm{p}$ process realized. Therefore, it provides convenience for programming, especially for those applications with varying structural parameters.

FTM is not only applicable to a periodic structure (superlattice) but also to isolated structures. For a certain isolated QD structure, increasing the periodic lengths reduces the crosstalk error, but increases the truncation error due to raised high-order Fourier harmonics. Practically, the number of Fourier harmonics that can be reserved is limited by the memory space of the computer. Consequently, proper periodic lengths have to be chosen in order to compromise the truncation error and the crosstalk error. As a rule of thumb, the periodic length in the computation for isolated QDs is usually employed as three times the value of the largest dimension of the QD structure, $N_{t r x}=N_{t r y}=N_{t r z}=7$ is chosen as the order of truncated Fourier harmonics for the pyramidal examples, and lower order truncated Fourier harmonics are applicable to other smooth structures.

This meshless FTM was developed on the zinc-blende III-V material systems with QD structure as the illustration example, whereas this method is also applicable for other material systems and other kind of herterostructures by its generality. 


\section{CHAPTER 4}

\section{ELECTRONIC STRUCTURES OF QDS}

In this chapter, the meshless FTM developed in Chapter 3 is applied to investigate electronic structures of QDs. The influence of dimension and strain on the electronic structures of InAs/GaAs QDs is studied, the effects of wetting layer on band structures of QDs are discussed as well, the mechanism of redshift in InAs/ $\operatorname{In}_{\mathrm{x}} \mathrm{Ga}_{(1-\mathrm{x})} \mathrm{As}$ QDs are investigated finally. The zinc blende QDs is assumed to be grown along normal direction [001], and the effect of piezoelectricity is neglected in this chapter. The material parameters adopted in this chapter are tabulated in Table $3-1$.

\subsection{Influence of dimension and strain}

The influences of dimension and strain on the band energies and the PDFs are investigated in this section through adopting a series of truncated pyramidal

QDs with $L_{x}=L_{y}=136 \AA$, and $h=60(1-f) \AA$, where the truncation factor $f=0,0.1$, $0.2,0.3,0.4,0.5,0.9$ denotes the extent of height truncation.

\subsubsection{Band-edge profiles}

Figure 4-1 shows the band-edge profiles of the pyramidal QD before (dash lines) and after (solid lines) the strain modification along the [001] [Figure 4-1(a)] and the [100] [Figure 4-1(b)] directions. The dominant strain effect is the hydrostatic pressure that enlarges the band-gaps and reduces the potential well in CB. The compressive hydrostatic strain component and the negative value of 
deformation constant $a_{c}$ (in Table 3-1) lead to the shift of $\mathrm{CB}$ to higher energy. However, the VBs after the strain modification become more complicated. It is seen in Figure 4-1(a) that the HH and LH potential wells deviate from each other spatially. The HH potential well skews toward the bottom of the pyramid, while the LH potential well notches down sharply at the tip of the pyramid due to the concentrated stress at the tip of the pyramidal QD with a sharp feature. This is not seen in Figure 4-2(a) for a half-truncated pyramidal QD due to the absence of the tip. Drastic modifications on $\mathrm{HH}$ and LH band-edge profiles at the four corners of the pyramid base are also expected due to stress concentration. These significant features of the band-edge profile of the pyramidal QD are consistent with that reported in and Ref. [41] and Ref. [59], which is done with the linear continuum elastic strain theory.

The band-edge profiles of the half-truncated pyramidal QD before (dash lines) and after (solid lines) the strain modification along the [001] direction and the [100] direction are plotted in Figure 4-2(a) and Figure 4-2(b), respectively. There is no significant difference between the plots in Figure 4-2(b) and Figure 41(b) due to the same pyramid base. Nevertheless, comparing the plots in Figure 42(a) and Figure 4-1(a), their situations are quite different due to the tip contrast. These typical characteristics of the half-truncated pyramidal QD are also in agreement with the one reported in Ref. [41]. 

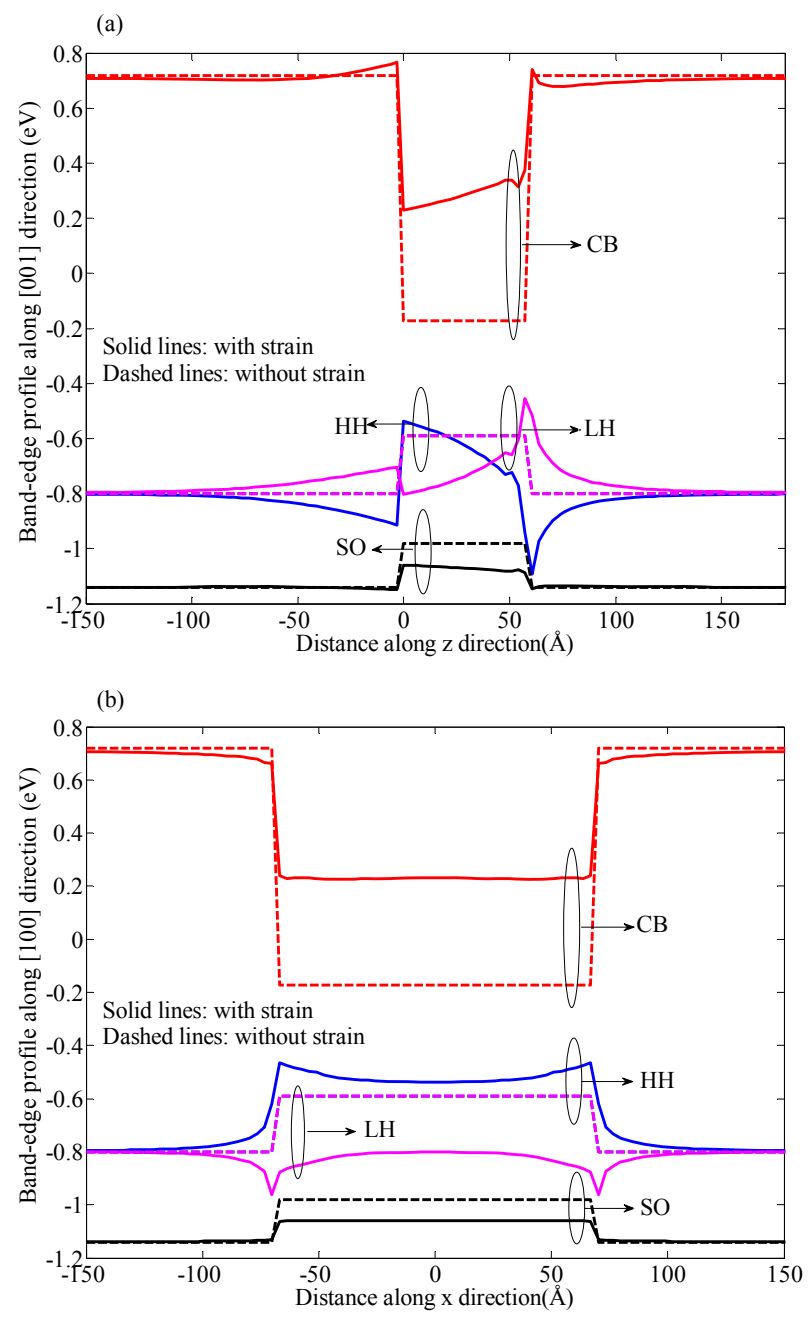

Figure 4-1 Band-edge profile of the pyramidal $Q D$ example with $L_{x}=136 \AA$, $L_{y}=136 A$ and $h=60 A$ along the [001] direction [Figure 4-1(a)] and the [100] direction [Figure 4-1(b)]. The dashed lines are the band-edge profiles before the strain modification and the solid lines are the band-edge profiles after the strain modification. 

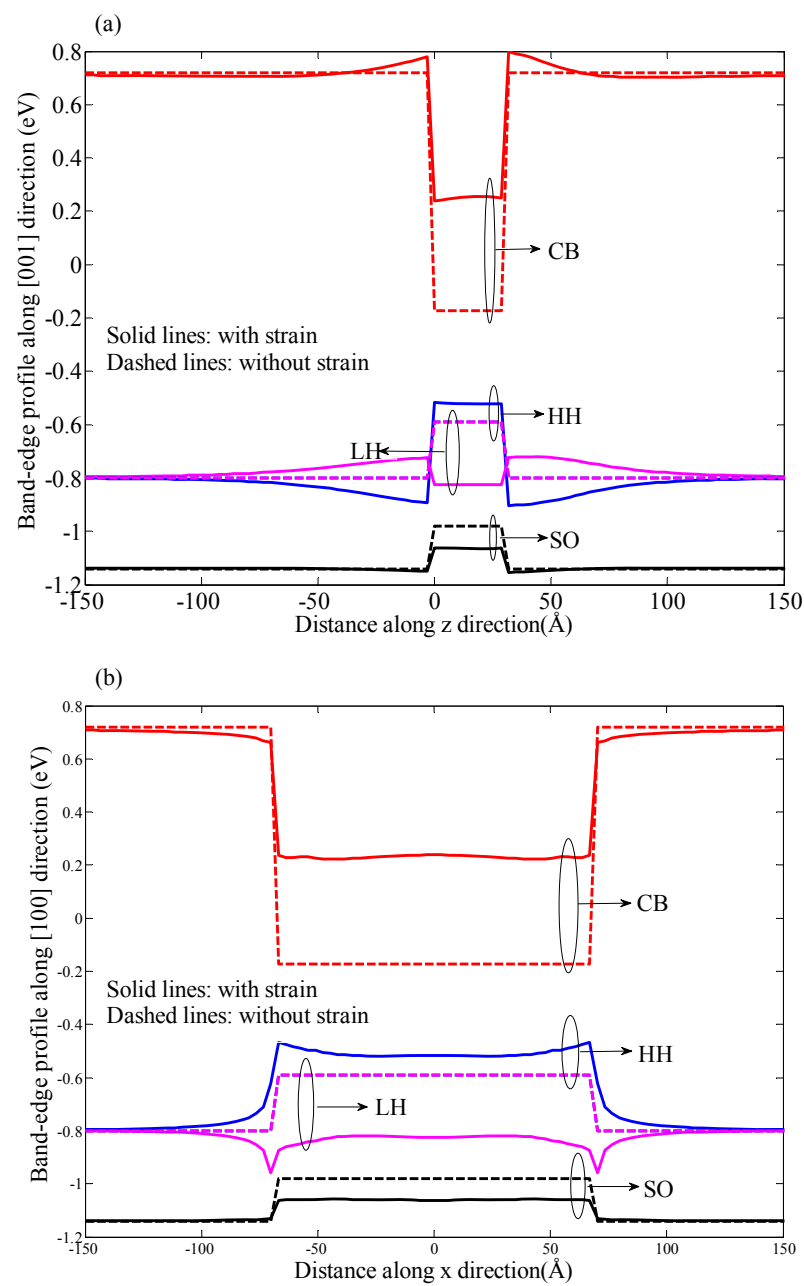

Figure 4-2 Band-edge profile of the half-truncated pyramidal $Q D$ example along the [001] direction [Figure 4-2(a)] and the [100] direction [Figure 4-2(b)]. The dashed lines are the band-edge profiles before the strain modification, and the solid lines are the band-edge profiles after the strain modification.

\subsubsection{Energy states}

Figure 4-3 shows the variation of ground-state energies (E1, HH1 and LH1) for the height truncation factor ranging from 0 to 0.9 . The increase of truncation factor implies the decrease of dimension, whereas the electron-hole ground-state energy gaps are enlarged upon the decrease of dimension, in agreement with the blue shift in smaller particles $[195,196]$. E1 is much more sensitive to the change of dimension than HH1 and LH1 due to smaller electron effective mass. The same 
phenomenon has been reported in an investigation of the influence of QD size on the ground-state energy[35].

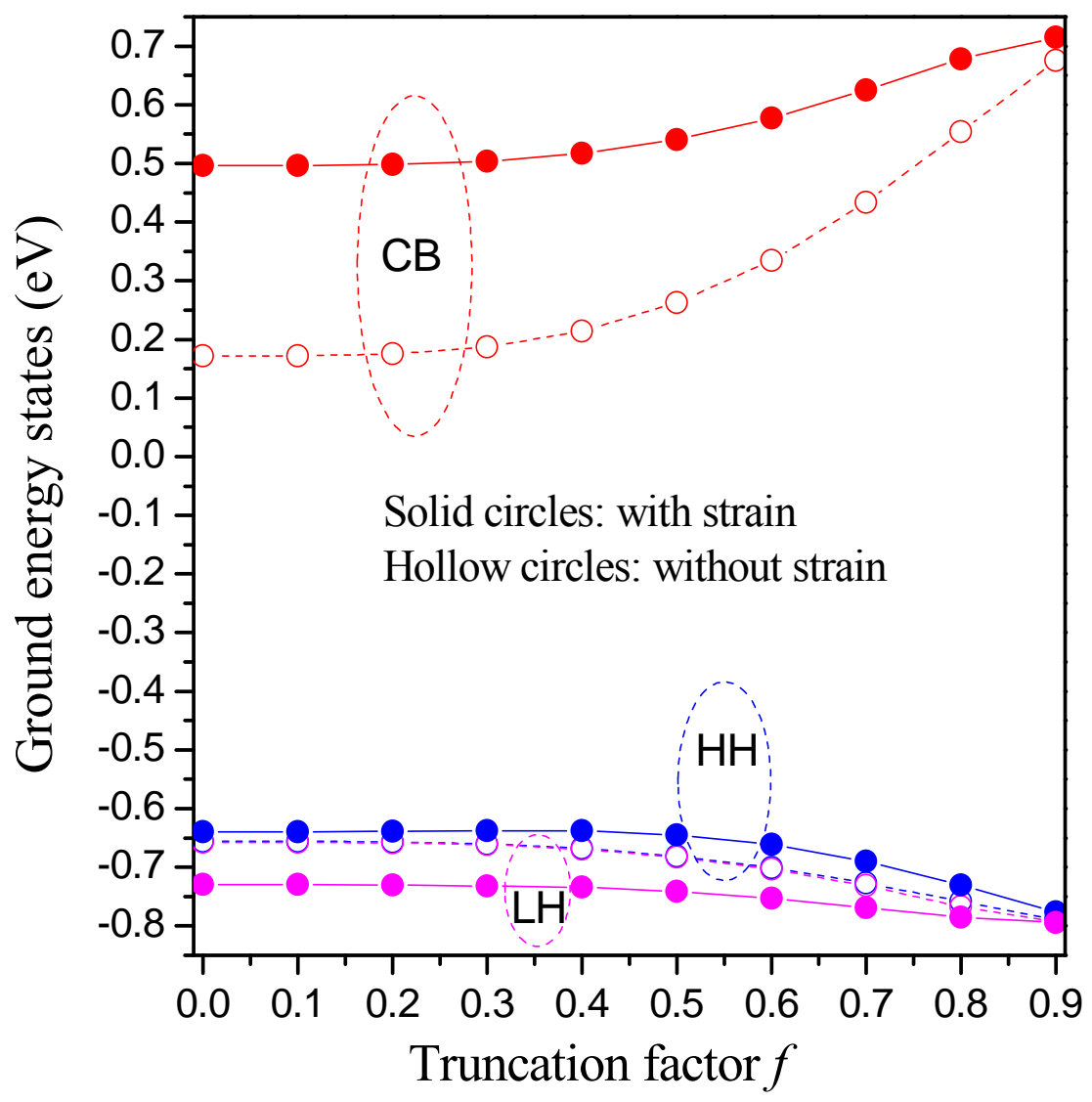

Figure 4-3 Ground-state energies of a series of truncated pyramidal QDs with (solid circles) and without (hollow circles) the strain consideration under different degree of height truncation.

Strain shifts energy levels in different extent for the structures with different truncation factors, which is demonstrated by the difference between the calculation results with and without strain consideration in Figure 4-3. The strain effect on E1 and LH1 is dominant at small $f$, or for a sharp pyramidal QD, whereas the confinement becomes more dependent on the dimension at large $f$ when the QD 
becomes flat. The more influential strain effect on E1 and LH1 at smaller $f$ is ascribed to the concentrated stress on the dot material as illustrated in Figure 4-1(a). The main contribution of the enlargement of the electron-hole ground-state energy gap is the variation of E1, in consistence with the conclusion of the empirical tightbinding work [27]. The stress becomes weakened as the sharp feature of the QD structure disappears, and thus the strain effect cannot generate the sharp potential well anymore as shown in Figure 4-2(a). The strain effect on HH1 is opposite to those on E1 and LH1 due to increased depth of $\mathrm{HH}$ potential well after strain modification. The strain effect on $\mathrm{HH} 1$ does not have much dependence of on $f$.

\subsubsection{Envelope functions}

Figure 4-4 gives intuitionistic description of the three-dimensional groundstate PDFs in the pyramidal QD ( $\mathrm{f}=0)$ with [Figure 4-4(a)] and without [Figure 44(b)] strain consideration. Each state is described by four PDF components in CB, $\mathrm{HH}, \mathrm{LH}$ and SO bands whose summation equals unity, but has a major component by which the state is denoted. It can be found that the CB portion of the electron ground state is increased due to the strain effect, i.e., from $78.17 \%$ to $87.29 \%$, denoting reduced band mixing between the $\mathrm{CB}$ and VBs due to the enlargement of the band-gaps illustrated in Figure 4-1. For the hole ground states, band mixing occurs mainly between $\mathrm{HH}$ and LH bands. Due to the strain, the band mixing between $\mathrm{HH}$ and $\mathrm{LH}$ bands is reduced for both HH1 and LH1 states. This reduction can be predicted from the separate localizations of $\mathrm{HH}$ and $\mathrm{LH}$ potential wells as illustrated by the crossover of $\mathrm{HH}$ and $\mathrm{LH}$ band edge profiles in Figure 41. As seen from the band-edge profiles and PDFs shown in Figure 4-2 and Figure $4-5$, this is also true in the truncated pyramidal QD $(f=0.5)$. In general, band mixing effect becomes less significant as a result of strain effect. 


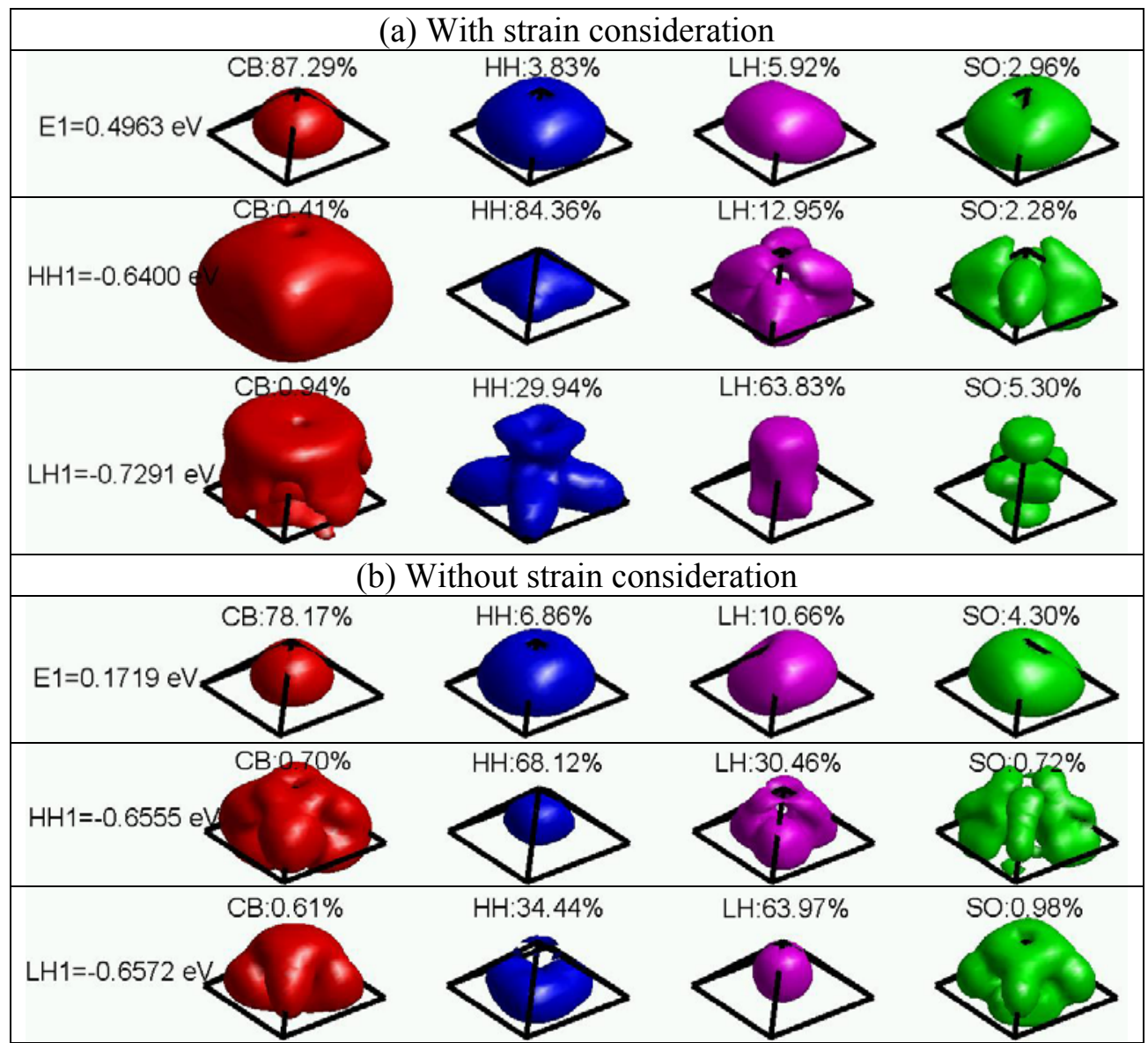

Figure 4-4 PDFs of the ground states of the pyramidal $Q D$. (a) with the strain consideration and (b) without the strain consideration.

In Figure 4-4, strain causes the PDF's HH component of the HH1 state to change from a bead to a flatted quatrefoil, which is in consistence with the shape change of the HH-band potential well shown in Figure 4-1. For the same reason, the PDF's LH component of the LH1 state is changed from a bead to a pillar. Unlike the squared $\mathrm{HH}$ wavefunction with $|000\rangle$ degeneracy in Ref. [58], four hollows arise in PDF's HH component of the HH1 state, such a difference is mainly due to the different strain computation methods as stated in Chapter 2. Nevertheless, corresponding modifications due to strain are not that much for the half-truncated pyramidal QD as illustrated in Figure 4-5, because of the less stress concentration. Since the sharp features of the CB potential well have no obvious 
change due to strain as shown in Figure 4-1and Figure 4-2, no distinct variations in the PDF's CB components of the E1 state can be visualized in Figure 4-4 and Figure 4-5. That is to say, the variation of PDF's CB components is unremarkable, whereas, the variation of PDF's HH component of the HH1 state and the variation of PDF's LH component of the LH1 state are on the contrary. This phenomenon can be explained as [41]: the smaller effective mass of electron induces the stiff envelop function of electron in the real space, whereas heavier mass of hole requires hole states to vary easily to adapt the potential fluctuations due to strain. Generally, the shape variations of the PDF's main components are determined by the change of the potential well shape due to stain modification and the mass magnitude of the carriers.

In the similar way, the location and the confinement of the ground states are affected by strain. This can be clearly seen in Figure 4-6, which plots the two cross-sectional views of the major PDF components of the three ground states of the pyramidal QD $(f=0)$. The black dash lines indicate the edge profile of the pyramidal shape. It is shown that the three ground states become poorly confined as illustrated by wider span with lower maxima of PDFs. The PDF of the LH1 state shows an obvious shift in location toward the pyramid tip due to the drastic change of the QW shape in the LH band. These phenomena are less obvious for the half-truncated pyramidal QD $(f=0.5)$ as shown in Figure 4-7, due to less stress concentration. 


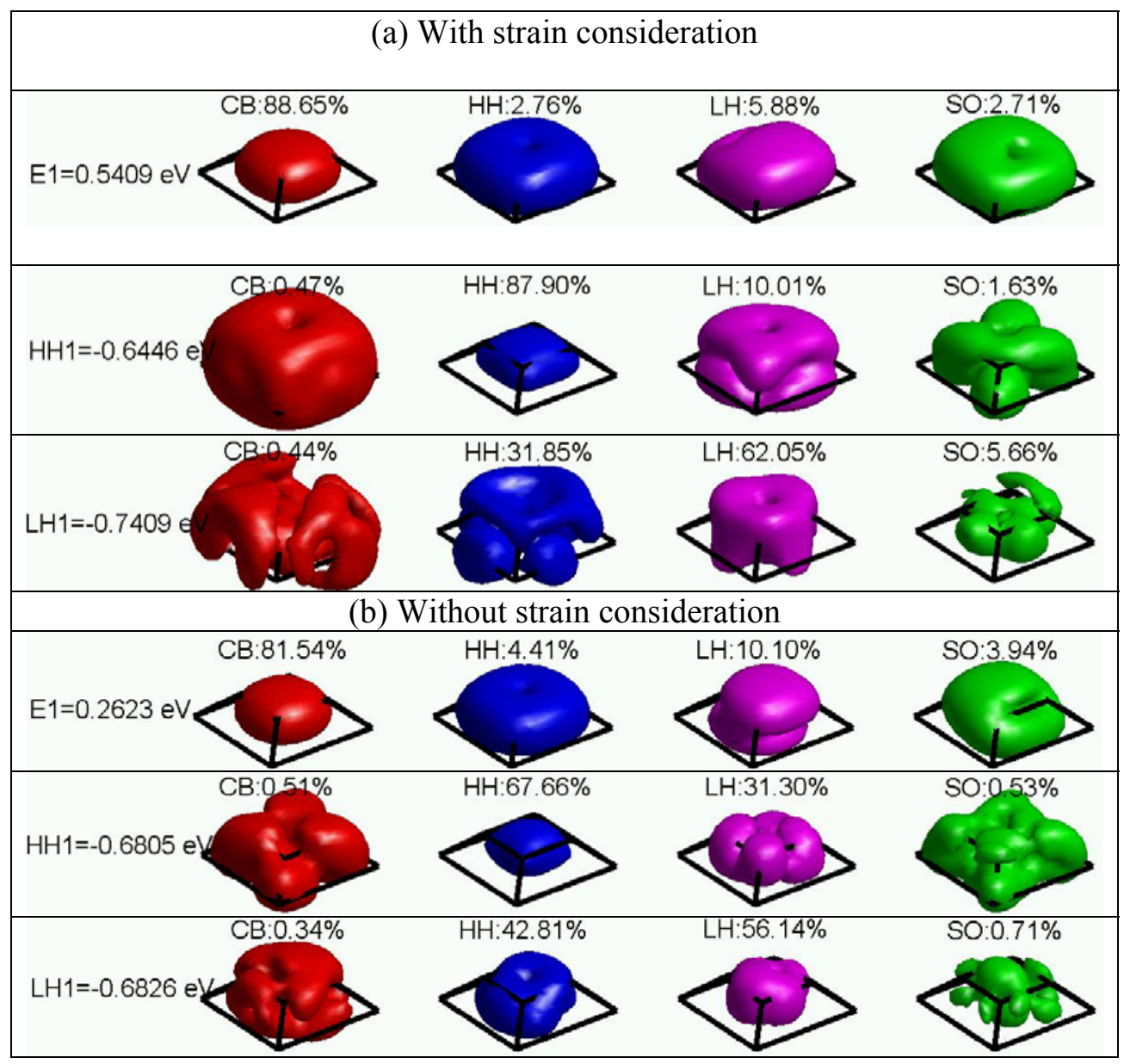

Figure 4-5. PDFs of the ground states of the half-truncated pyramidal QD. (a) with the strain consideration and (b) without the strain consideration. 


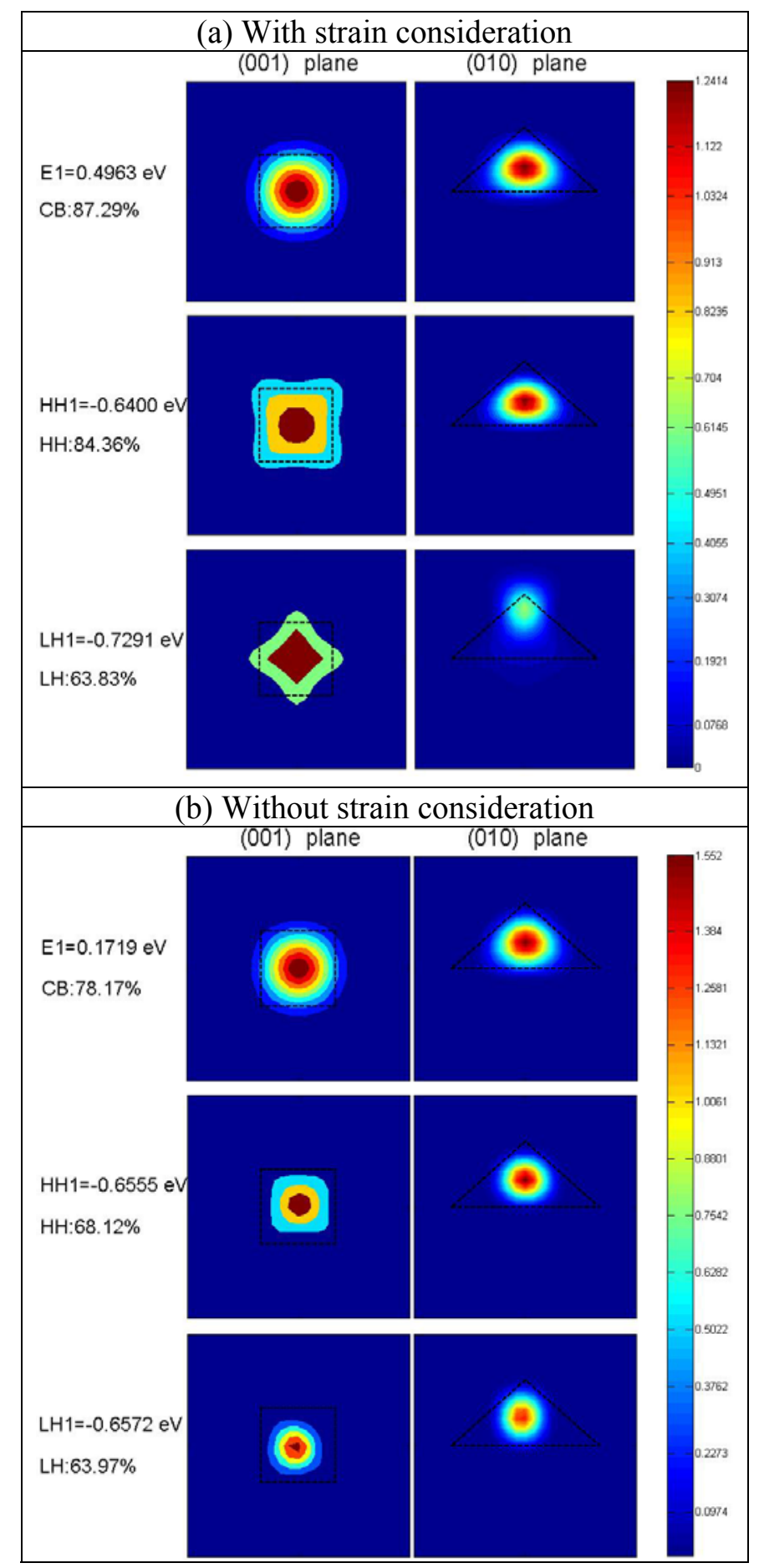

Figure 4-6. Major PDF components of the three ground states of the pyramidal $Q D$ across the (001) plane and the (010) plane: (a) with the strain consideration and (b)without the strain consideration. The dash lines make up the pyramidal shape section. 


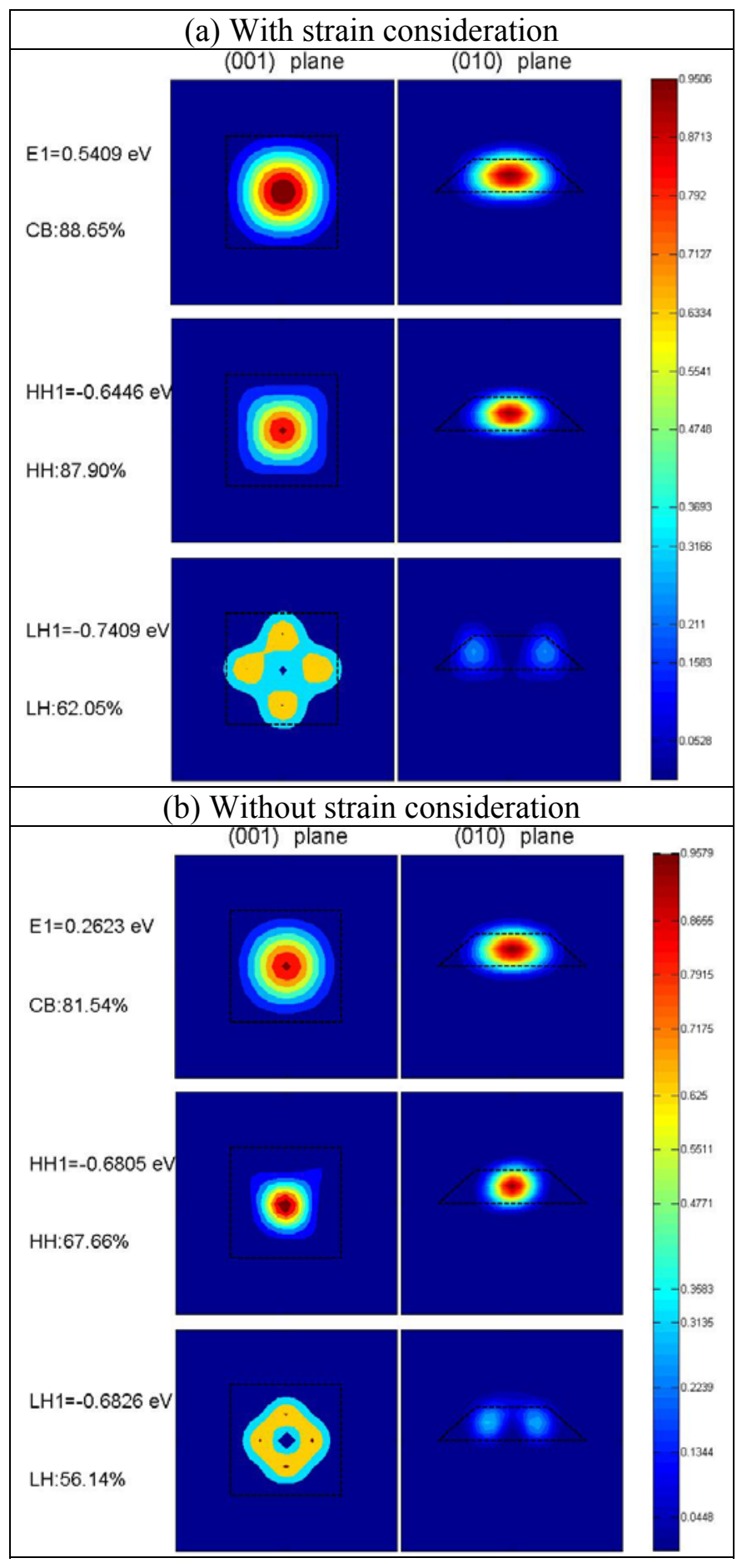

Figure 4-7 Major PDF components of the three ground states of the half-truncated pyramidal QD across the (001) plane and the (010) plane: (a) with the strain consideration and (b)without the strain consideration. The dash lines make up the pyramidal shape section. 


\subsection{Effect of wetting layer}

The effect of the wetting layer on the electronic structures of a series of truncated-pyramid InAs/GaAs QDs (shown in Figure 4-8) is studied in this section. Provided the same material is used, the critical thickness of the wetting layer is not affected by the height of the geometry, hence the wetting layer with a thickness of $0.5 \mathrm{~nm}$ was chosen for all the structures.

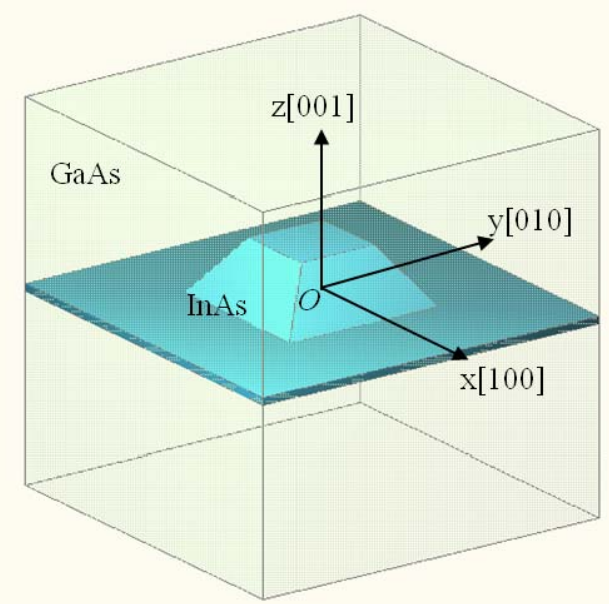

Figure 4-8 Geometric model of a truncated-pyramid InAs/GaAs QDW. The bottom dimension, the top dimension, and the height of dot are $12 \mathrm{~nm}, 12 \mathrm{fm}$, and $6(1-f)$ $\mathrm{nm}$, respectively. The thickness of the wetting layer is $0.5 \mathrm{~nm}$. $f$ is the height truncation factor.

The influence of the wetting layer on the ground-state energies are discussed through employing a series of truncated pyramidal QDs with different truncation factors $(f=0,0.1,0.2,0.3,0.4,0.5,0.6,0.7,0.8,0.9)$. For a clear illustration, the ground-state energies of a series of truncated-pyramidal InAs/GaAs QDWs and QDs with the same truncation factors are calculated. Figure 4-9 shows these ground-state energies E1 and HH1 of QDWs (solid lines) and QDs (dashed lines) under different truncation factors. It can be seen that the difference between the case of QDWs and the case of QDs is obvious only at a higher height truncation ( $\mathrm{f} \geq 0.5$ ), especially for $\mathrm{HH} 1$ due to the heavier effective mass of HHs. 
Therefore, a wetting layer effect becomes significant only as the thickness of the wetting layer is comparable with the height of the dot.

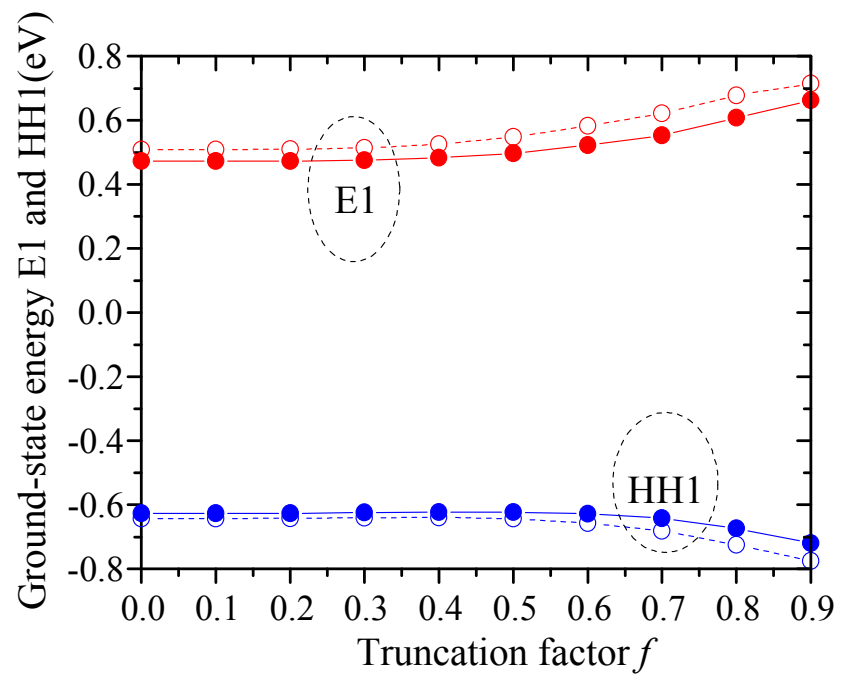

Figure 4-9 Ground-state energies E1 and HH1 of a series of truncated-pyramidal InAs/GaAs QDWs (solid lines) and a series of truncated-pyramidal QDs(dashed lines).

In order to further investigate the detailed information about the effect of wetting layer, the ground-state energies of the half-truncated QD $(f=0.5)$ with a cube just underneath its bottom (denoted as QDC) are computed. The thickness of the cube is also $0.5 \mathrm{~nm}$. The ground-state energies E1 and HH1 of QDC are tabulated in Table 4-1, together with those of the half-truncated QDW and the halftruncated QD. Figure 4-10 plots the strain components $e_{11}$ and $e_{33}$ of the halftruncated QD (dashed lines) and the half-truncated QDW (solid lines) along the [001] direction. The corresponding band-edge profiles of the half-truncated QD (dashed lines) and the half-truncated QDW (solid lines) along the [001] direction after strain modification are shown in Figure 4-11. Since the wetting layer only causes the change of the initial lattice mismatch at bottom of QDs, it redistributes the strain profile in the wetting layer region only rather than making so much change in the strain magnitude inside the dot area. Consequently, the wetting layer 
widens potential wells but it keeps positions of band edges inside the dot region almost unchanged. As a result, the ground-state energies E1 and HH1 of the halftruncated QDW in Table 4-1 vary significantly from those of the half-truncated QD, especially for the ground-state energy of electron E1 (up to $0.05 \mathrm{eV}$ ) owing to the smaller electron mass.

Table 4-1 Ground-state energies E1 and HH1 of the half-truncated QD, the QDC, and the half-truncated $Q D W$.

\begin{tabular}{cccc}
\hline \hline & \multicolumn{3}{c}{ Structures in Calculation } \\
Ground-state energies & Half-truncated & QDC & Half-truncated \\
& QD & QDW \\
E1 $(\mathrm{eV})$ & 0.5472 & 0.5099 & 0.4972 \\
$\mathrm{HH} 1(\mathrm{eV})$ & -0.6437 & -0.6308 & -0.6223 \\
\hline \hline
\end{tabular}

Additionally, it can be seen that the discrepancy of ground-state energies between the half-truncated QD and the QDC is much obvious than that between the half-truncated QDW and the QDC. This indicates that the main influence of the wetting layer on ground-state energy comes from the area just beneath the bottom of the dot. Figure 4-12(a) describes the major PDF components corresponding to ground-state energy E1 of the half-truncated QD and the half-truncated QDW. After the hybrid 2D/0D wetting layer confinement [197], the center of the PDF of the half-truncated QDW shifts towards the wetting layer region and a very small portion of PDF in the barrier region moves into the region just beneath the bottom of the dot but not into the whole wetting layer region. Consequently, the influence of the wetting layer on the electronic structures mainly comes from the region just beneath the bottom of the dot.

The major PDF components corresponding to ground-state energy HH1 of the half-truncated QD and the half-truncated QDW are shown in Figure 4-12(b). 
Similar to the results shown in Figure 4-12(a), the center of the PDF of the halftruncated QDW also moves towards the wetting layer region. The most significant difference in the PDFs of E1 and HH1 is that the PDF of HH1 are confined more obviously than the PDF of E1. This can be inferred from the smaller PDF volume and the bigger PDF value on the colorbar. As a result, a very small portion of the PDF of HH1 lies in the barrier region and, hence, the PDF portion that locates in the wetting layer is mainly from the dot region.

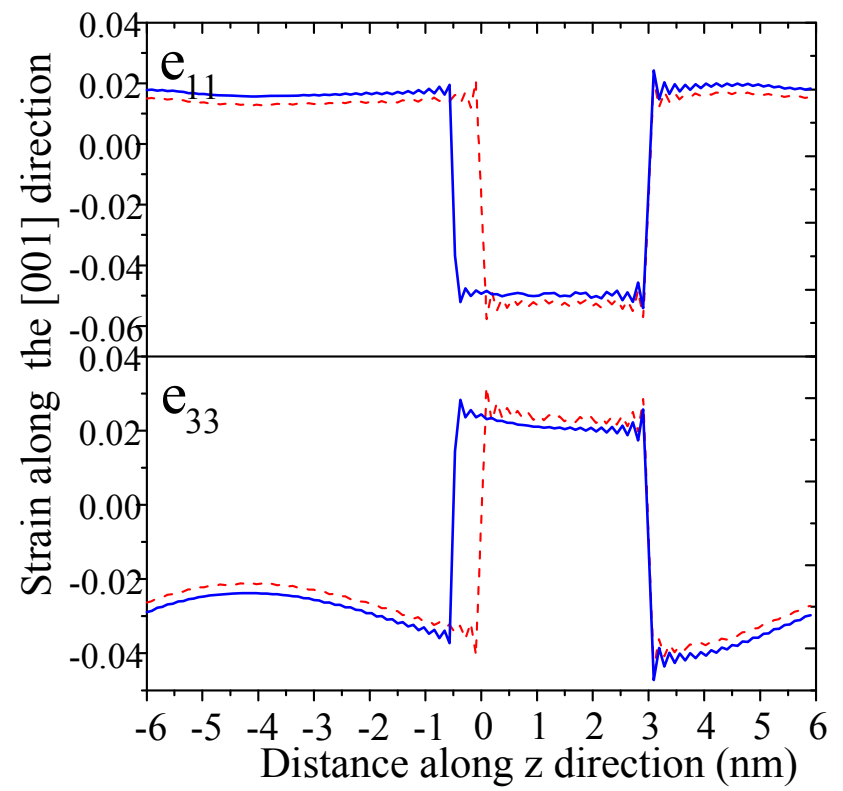

Figure 4-10 Strain components $e_{11}$ and $e_{33}$ of the half-truncated $Q D$ (dashed lines) and the half-truncated $Q D W$ (solid lines) along the [001] direction. 


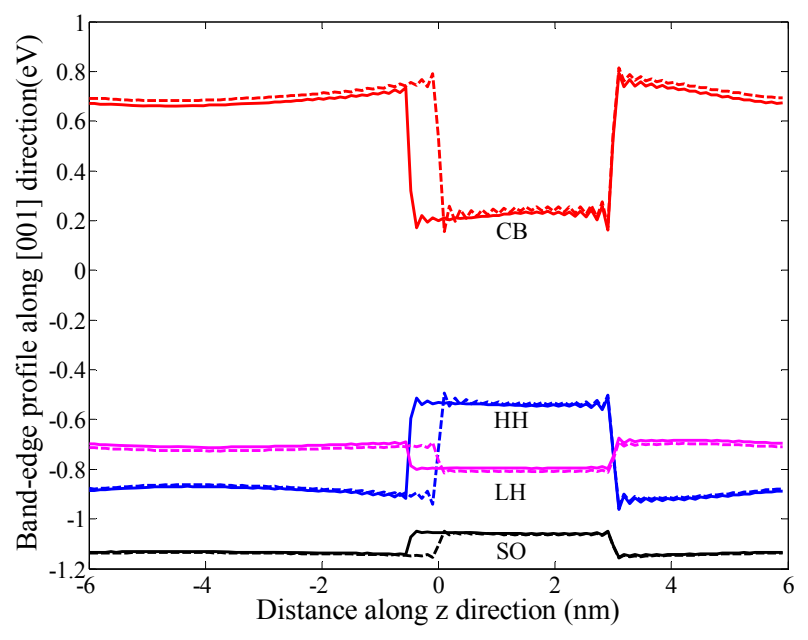

Figure 4-11 Band-edge profiles of the half-truncated QD (dashed lines) and the half-truncated $Q D W$ (solid lines) along the [001] direction

(a) QD

QDW

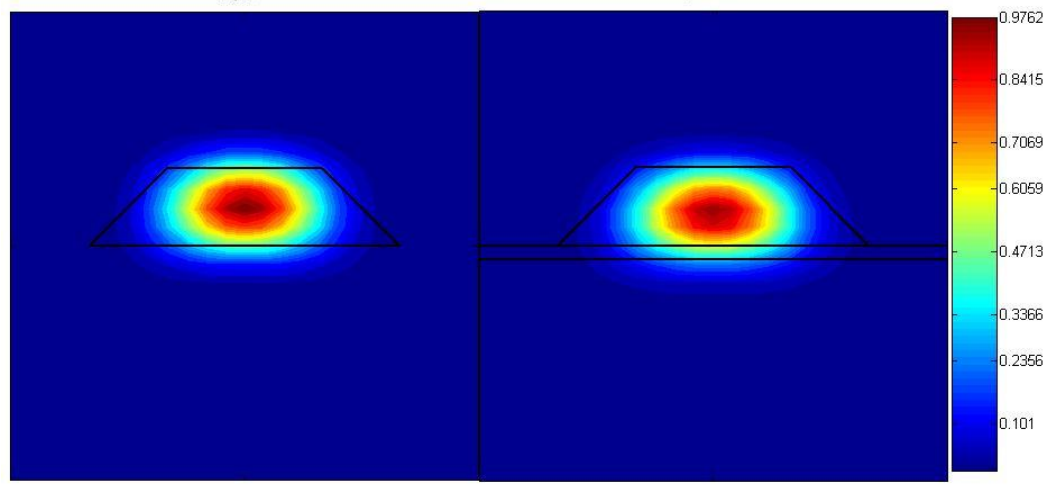

(b)

$Q D$

QDW

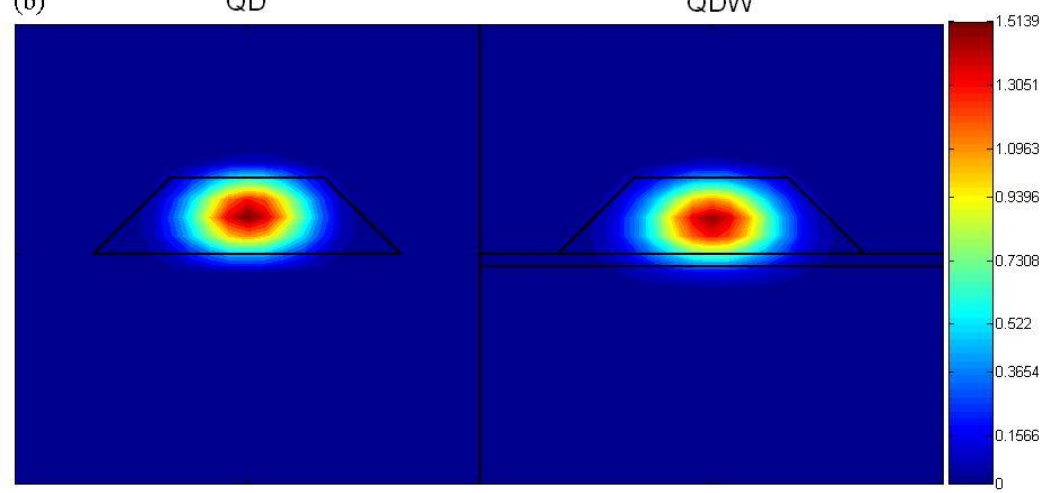

Figure 4-12 Major PDF components corresponding to ground-state energy E1 of the half-truncated $Q D$ and the half-truncated $Q D W$ [Figure 4-12(a)] and major $P D F$ components corresponding to ground-state energy HHI of the half-truncated $Q D$ and the half-truncated QDW [Figure 4-12(b)]. The black lines make up the shape section of the half-truncated $Q D$ and the half-truncated $Q D W$. 


\subsection{Redshift in $\operatorname{InAs} / \operatorname{In}_{\mathrm{x}} \mathbf{G a}_{(1-\mathrm{x})} \mathrm{As}$ QDs}

For the modeling structures, the truncated-conical shape structure are adopted, the dimension of the truncated-conical QD example follows those obtained by experimental measurements $[64,66]$. The diameter of the bottom is $21 \mathrm{~nm}(x \leq 0.33)$, the height is $4 \mathrm{~nm}$, the ratio of the bottom diameter and the top diameter is 3 , and the thickness of the wetting layer is $1.6 \mathrm{ML}$. A Cartesian coordinate is set up for convenient calculation: the origin is at the bottom center of QDs and the $z$ direction ([001]) is along the height of QDs. According to the calculation, the wavelength changes from $1.39 \mu \mathrm{m}$ to $1.49 \mu \mathrm{m}$ when the base dimension fluctuates between $17 \mathrm{~nm}$ and $25 \mathrm{~nm}(x=0.24)$.

\subsubsection{Selection of In composition}

Figure 4-13 describes how the initial lattice mismatch varies with the In composition $x$ and the thickness of LCL $t$. The initial lattice mismatch decreases rapidly as $x$ varies from 0 to 0.8 , whereas it weakens slowly with the increasing thickness of LCL, especially for a small $x$. The initial lattice mismatch locates in the range of $-0.067 \leq \varepsilon_{0} \leq-0.5$ as $x$ is situated in the area of $0 \leq x \leq 0.35$. Since a large lattice mismatch is preferable to form a thinner wetting layer in the SK epitaxial growth model, this should be one of reasons that all of the redshift in InAs/InGaAs QDs was realized under $0 \leq x \leq 0.35$ in Ref. $[64,66]$. In addition, it is reported that the diameter of the truncated-conic bottom jumps from $21 \pm 4 \mathrm{~nm}$ up to $29 \pm 4 \mathrm{~nm}$ when $x$ equals to 0.35 [66], this may be caused by increasing thickness of wetting layer due to large initial lattice constant. Park et al [198] also reported that the wetting layer causes the size fluctuations of QDs during the growth of QDs. 
On the other hand, band discontinuity certainly will be unremarkable when composition $x$ approaches to 1 . The band-edge profiles after strain modifications (without splitting between $\mathrm{HH}$ and $\mathrm{LH}$ ) under various In compositions and a fixed thickness of LCL $(t=220 \mathrm{~nm})$ are shown in Figure 4-14. As the composition $x$ alters from 0 to 0.5 , the band-gap between the $\mathrm{CB}$ and the VB reduces, and redshift emerges, accompanied by a waning band discontinuity. $x=0.33$ is a turning point, under which the band discontinuity of the VB almost vanishes. Therefore, the composition $x$ should be confined in the scope of $0 \leq x \leq 0.33$ to avoid serious size fluctuations and a small band discontinuity. This is consistent with the experimental results reported in Ref. [66].

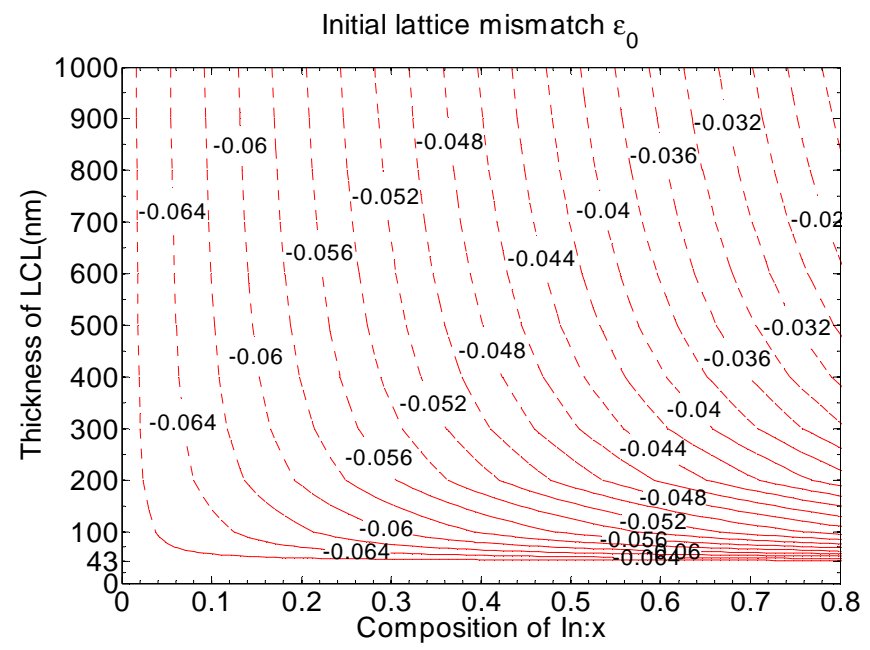

Figure 4-13 Initial lattice mismatch alters with the In composition $x$ and the thickness of $L C L$. 


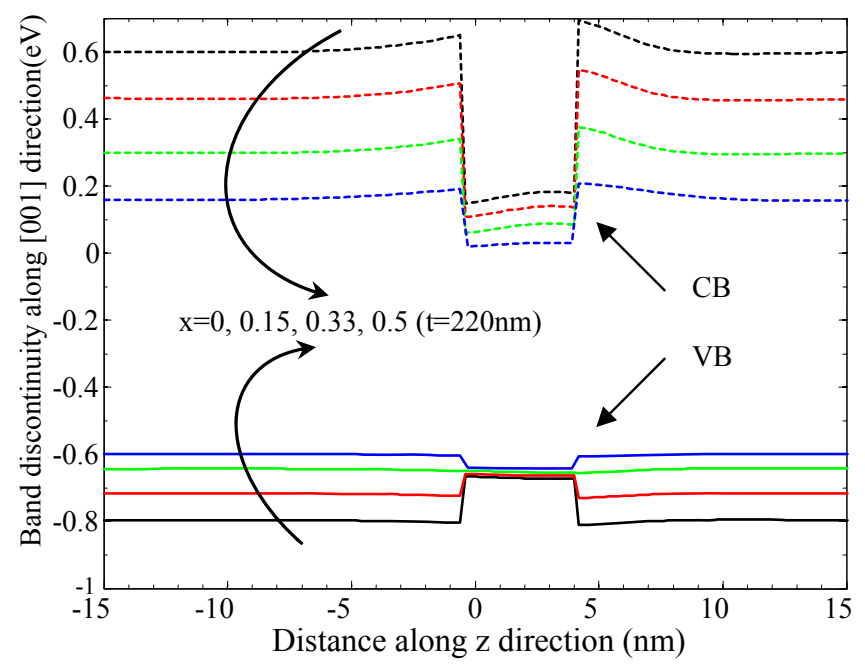

Figure 4-14 Band-edge profiles of the CB (dashed lines) and the VB (solid lines) after the strain modification (without the splitting between the HH band and the LH band) along the [001] direction (along the $z$ axis) vary with a series of In composition $x(x=0,0.15,0.33,0.5)$, and the LCL thickness $t$ is fixed to $220 \mathrm{~nm}$.

\subsubsection{Influence of LCL thickness and In composition}

Figure 4-15 shows the band-edge profiles after strain modifications (including the splitting of $\mathrm{HH}$ and $\mathrm{LH}$ ) under various thicknesses of LCL $t$ and a fixed $x(x=0.15)$. The band discontinuity enlarges but the band-gap decreases with the increase of thickness of LCL $t$. Therefore, a thicker LCL is a positive parameter for redshift and small thermal quenching, but the impact of the thickness of LCL $t$ is not distinctive. This indistinctive effect of the LCL thickness arises from the weak impact of the LCL thickness shown in Figure 4-13. Therefore, the next section mainly discusses the effect composition $x$ on the band discontinuity, strain effect, and ground-state energies. 


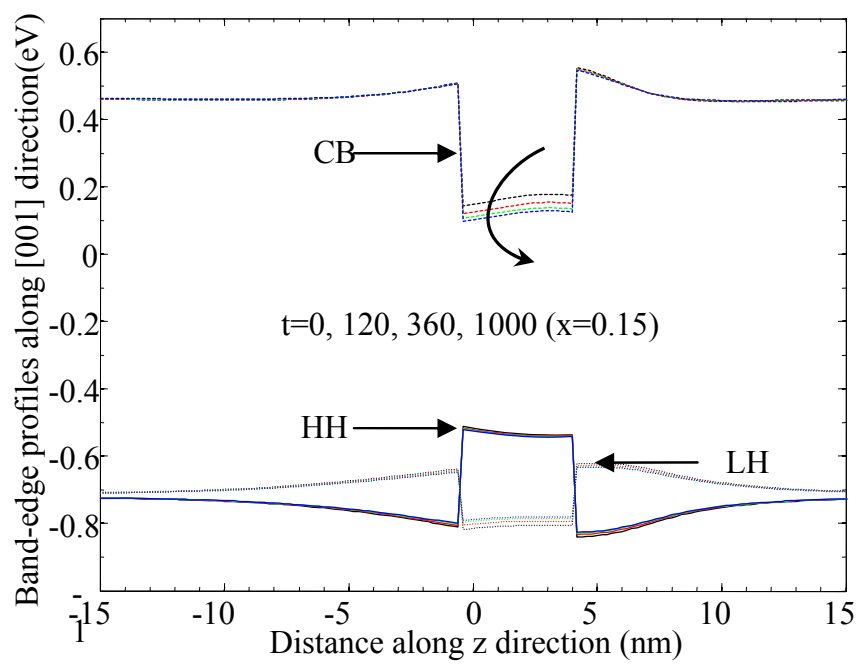

Figure 4-15 Band-edge profiles of the CB (dashed lines), the HH band (solid lines) and the LH band (dotted lines) after the strain modification (with the splitting between the HH band and the LH band) along the [001] direction (along the $z$ axis) vary with a series of LCL thicknesses $(t=0 \mathrm{~nm}, 120 \mathrm{~nm}, 360 \mathrm{~nm}$, and $1000 \mathrm{~nm})$, and the In composition $x$ is fixed to 0.15 .

Figure 4-16 presents band-edge profiles without strain modifications under various composition $x(x=0,0.15,0.33,0.5)$ and a fixed thickness of LCL $(t=220 \mathrm{~nm})$. In Figure 4-16, the band discontinuity drops with the increase of the In composition due to the decreased band-edges of InGaAs CL. In addition, the reduced strain due to the increase of In component modifies the band-edge of InAs to achieve redshift and further reduces the band discontinuity as shown in Figure 4-14. Consequently, the requirement of a larger redshift and a distinct band discontinuity is ambivalent, a proper In composition and a thicker LCL are used to mitigate this conflict. 


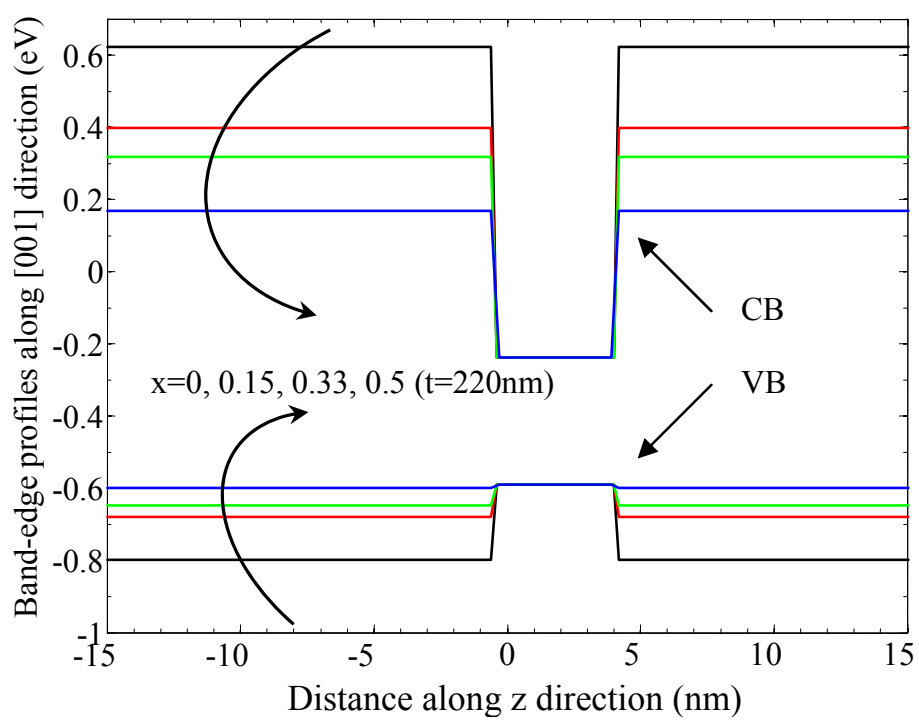

Figure 4-16 Band-edge profiles of the $C B$ (dashed lines) and the VB (solid lines) without the strain modification along the [001] direction (along the $z$ axis) vary with a series of In compositions $x(x=0,0.15,0.33,0.5)$, and the LCL thickness $t$ is fixed to $220 \mathrm{~nm}$.

The ground-state energies (E1, HH1, and LH1) of structures with $x=0,0.15$, $0.33,0.5$ and $t=220 \mathrm{~nm}$ are also calculated. Figure $4-17$ compares the ground-state energies without the strain effect (dashed lines) and those with strain consideration (solid lines). Strain effect can be denoted as the difference between solid lines and dashed lines, and compressive strain decreases with the increase of In composition and thus redshift. This is consistent with the decreased strain effect due to the increase of In composition in Figure 4-14. Variation of dashed lines due to increasing of In composition is not oblivious as that of solid lines, indicating that redshift related to band discontinuity is very unremarkable. This kind of redshift should be determined by several complex factors, therefore the unclear mechanism of this unremarkable redshift is ignored in this study. 


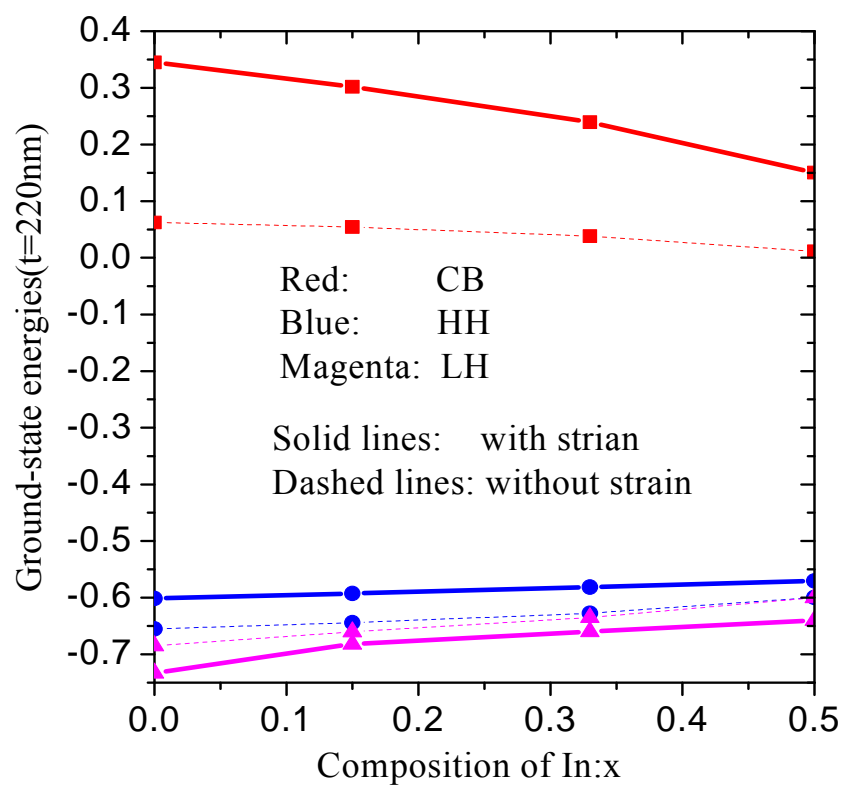

Figure 4-17 Ground-state energies vary with a series of In compositions $x(x=0$, $0.15,0.33,0.5)$, and the LCL thickness $t$ is fixed to $220 \mathrm{~nm}$. Solid lines represent the case with strain, and dashed lines denote the case without strain.

\subsection{Summary}

\subsubsection{Dimension effect and strain effect}

The dimension effect and the strain effect on QD's electronic structures have been investigated using FTM. The ground-state energy variation for a series of truncated pyramidal QDs shows that the electron state is the most sensitive to these effects due to the small effective mass of electron, whereas the strain effect on E1, LH1 and HH1 is more prominent for sharper QD.

The PDF study on a non-truncated and a half-truncated pyramidal QDs shows that the strain effect is exhibited in the following aspects 1) the reduction of band mixing between the $\mathrm{CB}$ and the $\mathrm{VB}, 2$ ) the reduction of band mixing between the $\mathrm{HH}$ band and the $\mathrm{LH}$ band, 3) the variation in the shape and the location of 
PDFs, 4) a weaker confinement to carriers, and 5) the major component of hole's state are more adapted to the fluctuation due to strain than the fluctuations in the major components of electron. These facts are explainable by the variation of potential wells due to the strain effect and the magnitude of the effective mass of carriers. The strain effect is less prominent in the half-truncated structure due to the absence of the pyramid tip and thus less stress concentration.

\subsubsection{Effect of the wetting layer}

The wetting layer effect becomes obvious only as the thickness of the wetting layer is comparable with the height of the QD. The confinement of the wetting layer changes the ground-state energy of electron energy up to $0.05 \mathrm{eV}$. Due to the different quantum confinement acting on the electron and the hole, the wetting layer drives a small portion of the PDF of E1from barrier region into wetting layer but drives a small portion of PDF of $\mathrm{HH} 1$ from the dot region into the wetting layer. The wetting layer also widens the potential well and, thus, reduces the energy band-gap. The main influence of the wetting layer on electronic structures is from the region just underneath the bottom of the truncated-pyramid dot. Therefore, this study supports the previous study done by Lee et al [68] very well and supplements the study from a very different point of view.

\subsubsection{Redshift in InAs/In $\operatorname{Ina}_{(1-x)} A s$ QDs}

Compared with InAs/GaAs QDs, the mechanism redshift in $\operatorname{InAs} / \operatorname{In}_{\mathrm{x}} \mathrm{Ga}_{(1-}$ ${ }_{x)}$ As QDs is that the shrinkage of the band gap due to strain decreases with introducing of In components in the GaAs CL material. However, the shrinkage of the band discontinuity, which leads to thermal quenching, is another outcome of gradually increased In component. A thicker LCL can be used to enhance the band 
discontinuity and further alleviate the enlargement of band gap although the impact of the LCL thickness is far away from that of the In composition. Therefore, a proper In composition and a thicker LCL is crucial to mediate this kind of contradiction. Other ways, such as adding an InAlAs CL can be used to increase the band discontinuity [199]. The consistency between the band-edge profiles and the ground-state energies demonstrates that the effect of strain on redshift becomes less obvious with an increase in the In component. The most proper range of In composition for redshift is $0 \leq x \leq 0.33$ to avoid size fluctuations and a small band discontinuity. 


\section{CHAPTER 5}

\section{ELIMINATION OF SPURIOUS SOLUTIONS}

In this Chapter, after recapturing the theorem of FTM with the SYM operator ordering (denoted as SYM FTM), the improved FTM with BF operator ordering (denoted as BF FTM) is developed. Several zinc blende and wurtzite quantum structure examples including QWs, QWRs are employed to demonstrate that the merging the cut-off method and the BF operator ordering is really a powerful approach to clean up the spurious solutions in both $\mathrm{CB}$ and VB. Screening of spurious solutions via Fourier truncation without any special treatment of interfaces and the cause of the spurious solutions under a large order of Fourier truncation are detailed as well.

\subsection{FTM with the BF operator ordering}

With the following basis,

$$
|S \uparrow\rangle,|X \uparrow\rangle,|Y \uparrow\rangle,|Z \uparrow\rangle,|S \downarrow\rangle,|X \downarrow\rangle,|Y \downarrow\rangle,|Z \downarrow\rangle,
$$

the eight-band $\mathrm{k} \cdot \mathrm{p}$ Hamiltonian for the zinc blende bulk crystal is written as [156],

$$
\mathbf{H}=\left(\begin{array}{cc}
\mathbf{H}_{4} & \mathbf{0} \\
\mathbf{0} & \mathbf{H}_{4}
\end{array}\right)+\mathbf{H}_{s o},
$$

where $\mathbf{H}_{s o}$ is spin-orbit Hamiltonian given in Eq. (4b) of Ref. [156]. As a direct consequence of the BF operator ordering, the block of $\mathbf{H}_{4}$ is expressed as [70-73], 


$$
\left(\begin{array}{cccc}
k A_{c} k+E_{c} & \frac{i}{2}\left(v_{1} k_{x}+k_{x} v_{2}\right) & \frac{i}{2}\left(v_{1} k_{y}+k_{y} v_{2}\right) & \frac{i}{2}\left(v_{1} k_{z}+k_{z} v_{2}\right) \\
-\frac{i}{2}\left(v_{2} k_{x}+k_{x} v_{1}\right) & H_{X X}+E_{v}-\frac{\Delta}{3} & k_{x} \tilde{N}_{+} k_{y}+k_{y} \tilde{N}_{-} k_{x} & k_{x} \tilde{N}_{+} k_{z}+k_{z} \tilde{N}_{-} k_{x} \\
-\frac{i}{2}\left(v_{2} k_{y}+k_{y} v_{1}\right) & k_{y} \tilde{N}_{+} k_{x}+k_{x} \tilde{N}_{-} k_{y} & H_{Y Y}+E_{v}-\frac{\Delta}{3} & k_{y} \tilde{N}_{+} k_{z}+k_{z} \tilde{N}_{-} k_{y} \\
-\frac{i}{2}\left(v_{2} k_{z}+k_{z} v_{1}\right) & k_{z} \tilde{N}_{+} k_{x}+k_{x} \tilde{N}_{-} k_{z} & k_{z} \tilde{N}_{+} k_{y}+k_{y} \tilde{N}_{-} k_{z} & H_{Z Z}+E_{v}-\frac{\Delta}{3}
\end{array}\right)
$$

where the inversion symmetry parameter $B$ is equal to zero $[107,153,154]$, and the split of the momentum matrix element $P$ in the coupling between the $\mathrm{CB}$ and the VB is $v_{1}=2 P$ and $v_{2}=P$, respectively [72]. The component comprising $A_{c}$ and $E_{c}$ is the scalar description of the $\mathrm{CB}$ and the typical diagonal component of the VB is,

$$
H_{X X}=k_{x} \tilde{L}^{\prime} k_{x}+k_{y} \tilde{M} k_{y}+k_{z} \tilde{M} k_{z}
$$

The other diagonal elements for the VB are given by the cyclic permutation of $x, y$, and $z . \tilde{N}_{+}$and $\tilde{N}_{-}$are the split of $\tilde{N}$ with the relationship,

$$
\tilde{N}^{\prime}=\tilde{N}_{+}+\tilde{N}_{-}
$$

where $\tilde{N}_{-}$is estimated as,

$$
\tilde{N}_{-}=M-\frac{\hbar^{2}}{2 m_{0}}
$$

As $v_{1}=v_{2}=P$ and $\tilde{N}_{+}=\tilde{N}_{-}=\frac{\tilde{N}^{\prime}}{2}$, the BF operator ordering becomes the SYM operator ordering. $E_{c}$ and $E_{v}$ represent the $\mathrm{CB}$ and VB band edges, respectively; $\Delta$ is the spin-orbit splitting; and $\tilde{L}^{\prime}, \tilde{M}$, and $\tilde{N}^{\prime}$ are the renormalized Kane parameters and expressed with the modified Luttinger parameters using [73], 


$$
\begin{aligned}
& \tilde{L}^{\prime}=-\frac{\hbar^{2}}{2 m_{0}}\left(\tilde{\gamma}_{1}+4 \tilde{\gamma}_{2}\right), \\
& \tilde{M}=-\frac{\hbar^{2}}{2 m_{0}}\left(\tilde{\gamma}_{1}-2 \tilde{\gamma}_{2}\right), \\
& \tilde{N}^{\prime}=-\frac{\hbar^{2}}{2 m_{0}} 6 \tilde{\gamma}_{3} .
\end{aligned}
$$

In the eight-band computation, the modified Luttinger parameters $\tilde{\gamma}_{i}$ are subtracted Luttinger parameters $\gamma_{i}$ with [194],

$$
\begin{aligned}
& \tilde{\gamma}_{1}=\gamma_{1}-\frac{E_{p}}{3 E_{g}}, \\
& \tilde{\gamma}_{2}=\gamma_{2}-\frac{E_{p}}{3 E_{g}}, \\
& \tilde{\gamma}_{3}=\gamma_{3}-\frac{E_{p}}{6 E_{g}},
\end{aligned}
$$

where $E_{g}$ represents the band gap.

The renormalized CB effective mass parameter $A_{c}$ is a tunable parameter and the following expression is adopted in this article,

$$
A_{c}=\frac{\hbar^{2}}{2 m_{c}^{*}}-\frac{2}{3} \frac{P^{2}}{E_{g}}-\frac{P^{2}}{3\left(E_{g}+\Delta\right)} .
$$

The bulk Hamiltonian matrix is applicable to heterosturctures as $k_{i}=-\nabla_{i}$. With BF operator ordering, the envelope function for the heterostructures is solved by the following eigen equation,

$$
\mathbf{H}(\mathbf{r} ; \mathbf{k}) \mathbf{F}(\mathbf{r})=E(\mathbf{k}) \mathbf{F}(\mathbf{r}),
$$

where $k_{i}$ is replaced with $\hat{k}_{i}$ and Hamiltonian is,

$$
\mathbf{H}=\sum_{i, j} \hat{k}_{i} H_{i j}^{(2)}(\mathbf{r}) \hat{k}_{j}+\sum_{i}\left[H_{i, L}^{(1)}(\mathbf{r}) \hat{k}_{i}+\hat{k}_{i} H_{i ; R}^{(1)}(\mathbf{r})\right]+H^{(0)}(\mathbf{r}) .
$$

The envelope function can be expressed in plane-wave expansion along the quantum confined direction(s) [60], 


$$
\mathbf{F}(\mathbf{r})=\left\{\begin{array}{ll}
e^{i k_{x} x+i k_{y} y+i k_{z} z} \frac{1}{\sqrt{d_{z}}} \sum_{n_{z}} \mathbf{c}_{n_{z}} e^{i n_{z} \kappa_{z} z} & \text { for QW } \\
e^{i k_{x} x+i k_{y} y+i k_{z} z} \frac{1}{\sqrt{d_{x} d_{y}}} \sum_{n_{x}} \sum_{n_{y}} \mathbf{c}_{n_{x} n_{y}} e^{i\left(n_{x} \kappa_{x} x+n_{y} \kappa_{y} y\right)} & \text { for QWR }
\end{array},\right.
$$

where $d_{\alpha}$ denotes the periodicity, $\kappa_{\alpha}=\frac{2 \pi}{d_{\alpha}},-\frac{\kappa_{\alpha}}{2}<k_{\alpha}<\frac{\kappa_{\alpha}}{2}, \alpha$ is the suffix representing directions in Cartesian coordination system, i.e., $x, y, z$, and $\mathbf{c}_{\mathbf{n}}=\left[c_{1} c_{2} \ldots c_{8}\right]_{\mathbf{n}}^{T}$. If there are $N$ superlattice periods, the Born-Von Karman cyclic boundary condition [99] gives,

$$
k_{\alpha}=\frac{2 \pi j}{N d_{\alpha}}, 0 \leq j \leq N .
$$

Eq. (5.10) can be completely handled in Fourier domain with Fourier series of $\mathrm{H}(\mathrm{r} ; \mathrm{k})$ [60]. This approach takes advantage of orthogonality of complex componential functions to evade the cumbersome integral calculation and presents the equation for eigen problem as,

$$
\left[\mathbf{M}_{u m, v n}\right]\left[\mathbf{c}_{v n}\right]=E\left[\mathbf{c}_{v n}\right]
$$

where the Hamiltonian matrix in Fourier domain, $\mathbf{M}_{u m, v n}$, is expressed with Fourier series $\tilde{H}^{(\alpha \beta)}, \tilde{H}_{L}^{(\alpha)}, \tilde{H}_{R}^{(\alpha)}$ and $\tilde{H}^{(0)}$ of the k·p Hamiltonian in the real space,

$$
\begin{aligned}
\mathbf{M}_{u m, v n} & =\sum_{\alpha, \beta} \tilde{H}_{u v, q_{i}=m_{i}-n_{i}}^{(\alpha \beta)}\left(k_{\alpha}+m_{\alpha} \kappa_{\alpha}\right)\left(k_{\beta}+n_{\beta} \kappa_{\beta}\right) \\
& +\sum_{\alpha} \tilde{H}_{u v, q_{i}=m_{i}-n_{i}, L}^{(\alpha)}\left(k_{\alpha}+n_{\alpha} \kappa_{\alpha}\right)+\sum_{\alpha} \tilde{H}_{u v, q_{i}=m_{i}-n_{i}, R}^{(\alpha)}\left(k_{\alpha}+m_{\alpha} \kappa_{\alpha}\right), \\
& +\tilde{H}_{u v, q_{i}=m_{i}-n_{i}}^{(0)}
\end{aligned}
$$

where $\beta$ is also a suffix similar to $\alpha$. The derivation is presented with QW as an example in Appendix E.

The dimension of the Fourier-domain Hamiltonian $\mathbf{M}$ is only determined by the order of the Fourier frequencies of $\mathbf{H}$. An arbitrary heterostructure may 
result in an infinite dimension of $\mathbf{M}$, but practically, the Fourier spectrum has negligible magnitudes at high frequencies and, thus, can be truncated. If terms up to $N^{\text {th }}$ order in Fourier series are retained in the wavefunction, i.e., $n_{\alpha}, m_{\alpha}=-N_{\alpha}, \cdots, 0, \cdots N_{\alpha}$, the Fourier series terms of $\mathbf{H}$ will be reserved up to the $2 N_{\alpha}^{\text {th }}$ order, i.e., $q_{\alpha}=m_{\alpha}-n_{\alpha}=-2 N_{\alpha}, \cdots, 0, \cdots 2 N_{\alpha}\left(N_{\alpha}<\frac{n_{\alpha}}{4}\right.$ is used to serve the demand of the Nyquist sampling theorem). Therefore, this kind of truncation leads to the inborn cut-off characteristic of FTM, and the dimension of $\mathbf{M}$ is only determined by the order of Fourier truncation $N_{\alpha}$, i.e. $V \prod_{\alpha}\left(2 N_{\alpha}+1\right) \times V \prod_{\alpha}\left(2 N_{\alpha}+1\right)$, where $V$ is the number of bands involved in the calculation. Of course, splitting the first order Hamiltonian into two parts results in a larger computation volume for the BF FTM compared with the SYM FTM.

The plane-wave expansion is a commonly-adopted approach for the $\mathrm{k} \cdot \mathrm{p}$ method. The wave basis cut-off is inborn with plane-wave expansion based methods. In contrast to most works that solved eigen problems in real space, this approach handles the equation in the Fourier domain, via Fourier transforming the spatial-domain Hamiltonian matrix to the Fourier domain using the orthogonal feature of the basis functions of the Fourier representation. Since high-order Fourier components of $\mathbf{H}$ are truncated in our computation, "Fourier truncation" in this circumstance has two aspects: truncation of high-order Fourier components of $\mathbf{H}$ and in parallel with the wave basis cut-off for the plane-wave expansion, which is far more than the simple wave-basis cut-off investigated in previous reports.

The formulation of FTM with the BF operator ordering is illustrated with zinc blende crystals. However, Eq. (5.15) is also applicable to wurtzite crystals, even for the Hamiltonian written in other bases. Though the strain effect is not 
discussed in this chapter, the strain Hamiltonian with the BF operator ordering in the momentum space can be derived by applying the above procedure on the Eq. (25) in Ref. [107].

\subsection{Results and discussions}

Comparison between the results of the SYM operator ordering and the BF operator ordering has been carried out with FEM by Veprek et al [73]. They also reported that spurious solutions turn up in the usually unaffected six-band $k \cdot p$ model. They surmised that these unwonted spurious solutions may cause the rare adoption of FEM, in contrast to the widely used FDM and the plane-wave expansion methods. Consequently, it is necessary to see whether the spurious solutions turn up in the six-band $\mathrm{k} \cdot \mathrm{p}$ model realized by the very different FTM based on the plane-wave expansion of spatial Hamiltonian. Furthermore, the order of Fourier truncation $N_{t r}$ is a vital factor in the FTM computation for the eight-band $\mathrm{k} \cdot \mathrm{p}$ Hamiltonian, as $N_{t r}$ not only controls the accuracy of computation but also can be used to screen away spurious solutions. Therefore, Figure 5-1 plots the first four VB band structures of a $50 \AA$ InAs/GaAs zinc blende QW under various $N_{t r}$, where the computation is carried out using both the six-band SYM FTM [Figure 5-1(a)] and the six-band BF FTM [Figure 5-1(b)], and the material parameters are shown in Table 5-1. It can be seen in Figure 5-1(a) that the solutions are not stable for varying $N_{t r}$ and spurious solutions arise at the large- $k$, in accompany with oscillatory wavefunctions (not shown here). In contrast, all solutions in Figure 51(b) obtained with the BF operator ordering are consistent as $N_{t r}$ varies from 20 to 127. As shown by these results in the six-band $k \cdot p$ calculation for QWs, BF FTM performs well for rejecting spurious solutions without any control of $N_{t r}$, however, 
there is no convergence among the solutions of SYM FTM for varying $N_{t r}$.

Existence of such unwonted spurious solutions in both FTM and FEM may suggest that these spurious solutions are not subject to FEM, but to the envelope equations.
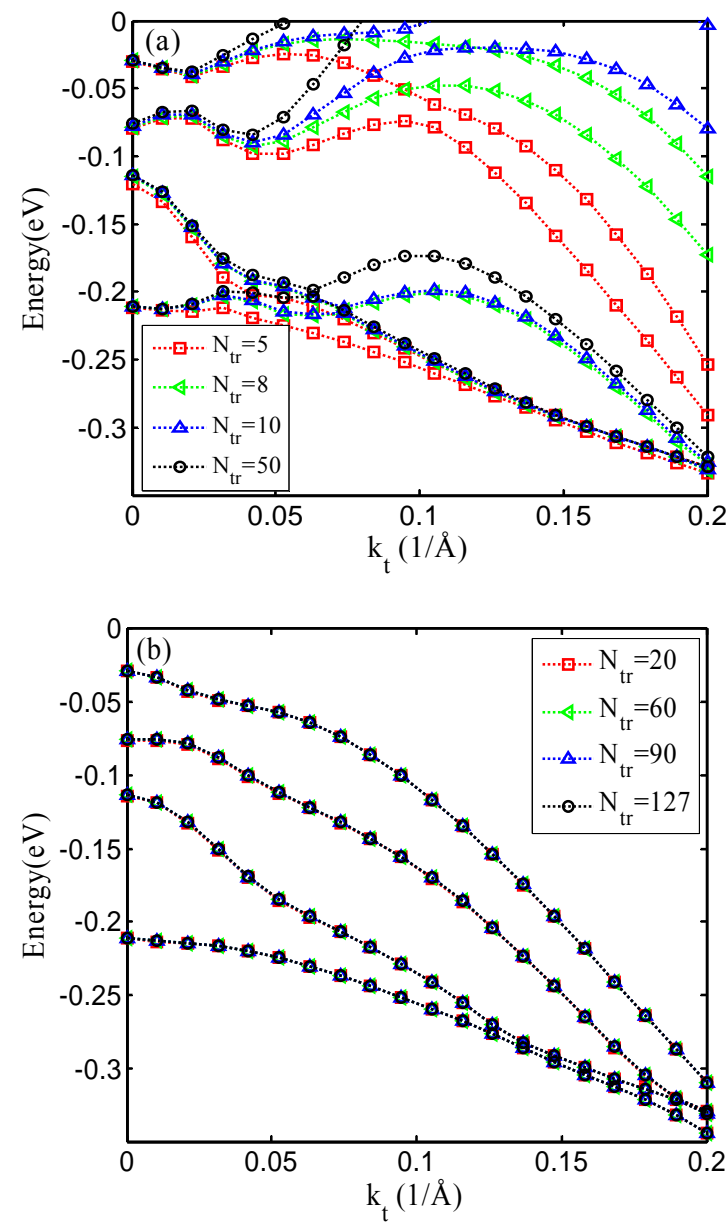

Figure 5-1 Energy band diagrams of a 50 A InAs/GaAs QW obtained using the sixband Hamiltonian matrix varies with various order of Fourier truncation $N_{t r}:$ (a) with the SYM operator ordering and (b) with the BF operator ordering.

Table 5-1 Kane parameters for the six-band calculation. These parameters are from Ref. [73], in units of $\hbar^{2} / 2 m_{0}$.

\begin{tabular}{lccccc}
\hline \hline Material & $\tilde{L}^{\prime}$ & $\tilde{M}$ & $\tilde{N}^{\prime}$ & $N_{+}$ & $N_{-}$ \\
InAs & -53.60 & -3.80 & -54.60 & -49.80 & -4.80 \\
GaAs & -15.25 & -2.65 & -17.40 & -13.75 & -3.65 \\
\hline \hline
\end{tabular}

As presented in Eq. (5.3), the most significant feature of the BF operator ordering is the nonuniform splitting of momentum matrix $P$ and the renormalized 
Kane parameter $\tilde{N}^{\prime}$. The nonuniform splitting of $P$ acts on the coupling between the $\mathrm{CB}$ and the $\mathrm{VB}$, while the nonuniform splitting of $\tilde{N}^{\prime}$ on the coupling in the VB. Nevertheless, there is no such a similar nonuniform splitting of $A_{c}$ for the $\mathrm{CB}$ component. Therefore, it is easily predicted that the BF operator ordering would not make the energy states in the $\mathrm{CB}$ much different from those obtained using the SYM operator ordering, especially for the QW heterostructure with quantization occurring in only one dimension. The above $50 \AA$ InAs/GaAs zinc blende QW is employed again. The results of the eight-band SYM FTM and the eight-band BF FTM are compared, where the material parameters are tabulated in Table 5-2. The fact is exactly that the CB energy dispersion obtained from the SYM FTM is totally in consistence with that from the BF FTM regardless of the choice of $N_{t r}$, even the same for the VB energy dispersion. This phenomena are very similar to that reported by Veprek et al [73]. Figure 5-2 only shows the eight-band BF FTM solutions for $N_{t r}=20,106,107,127$. As $N_{t r}$ varies from 20 to 127 , no spurious solutions appears in the $\mathrm{VB}$, while spurious solutions emerge in the $\mathrm{CB}$ since $N_{t r}=107$. That indicates that spurious solutions in the eight-band calculation for the heterostructures with $1 \mathrm{D}$ quantization tends to locate in the $\mathrm{CB}$ band. The $\mathrm{BF}$ operator ordering fails to resist spurious solutions, this may be caused by the coupling between the $\mathrm{CB}$ and the VB. In such a situation, it depends on the choice of $N_{t r}$, i.e. cut-off high order Fourier frequencies, to makes up the deficiency of the BF operator ordering in avoiding the spurious solution issue for the eight-band $\mathrm{k} \cdot \mathrm{p}$ computation for QWs. The most significant merit of such cut-off in FTM is that there is no requirement for manipulating material parameters or setting specific boundary conditions. 
Table 5-2 Material parameters used for the eight-band calculation. These parameter are cited after Ref. [73]. Except unit of Eg and $\Delta$ are $\mathrm{eV}$, other parameters are in the unit of $\hbar^{2} / 2 m_{0}$

\begin{tabular}{lccccccccc}
\hline \hline Material & $\tilde{L}^{\prime}$ & $\tilde{M}$ & $\tilde{N}^{\prime}$ & $N_{+}$ & $N_{-}$ & $A_{c}$ & $E_{p}$ & $E_{g}$ & $\Delta$ \\
InAs & -0.74 & -3.80 & -1.74 & 3.06 & -4.80 & -1.01 & 22.20 & .042 & 0.38 \\
GaAs & 1.67 & -2.65 & -0.48 & 3.17 & -3.65 & -0.96 & 25.70 & 1.52 & 0.34 \\
\hline \hline
\end{tabular}

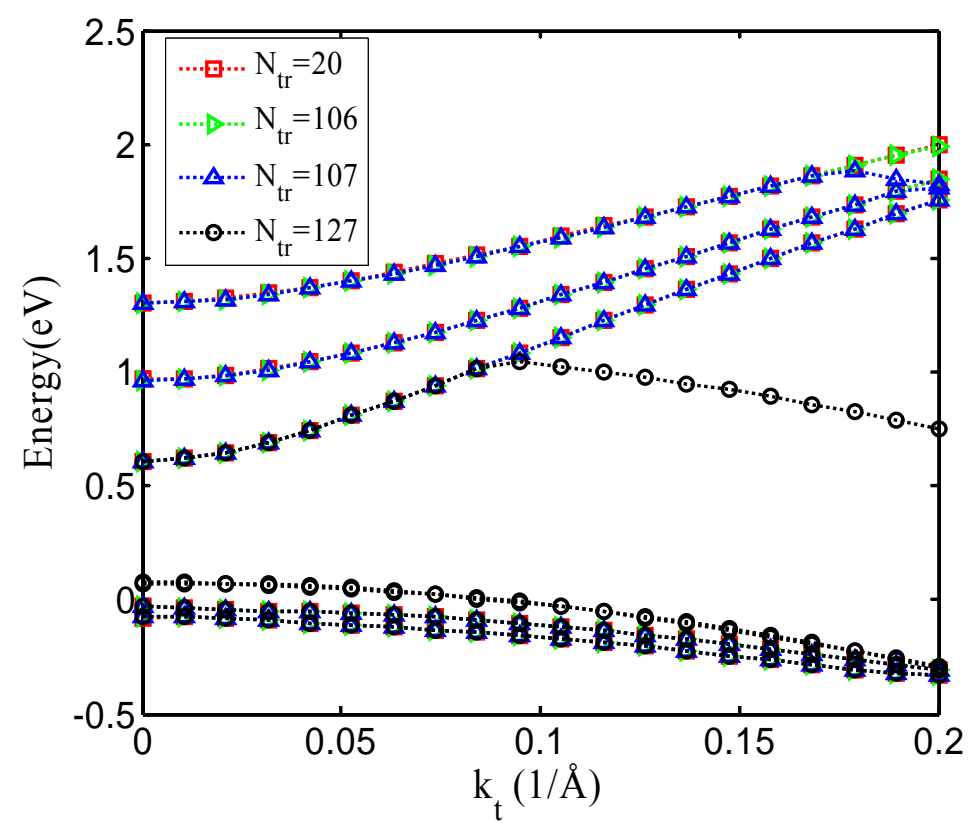

Figure 5-2 Energy band diagrams of a 50 A InAs/GaAs $Q W$ obtained using the eight-band Hamiltonian matrix and the BF operator ordering varies with various order of Fourier truncation $N_{t r}$.

The energy eigenvalues of a $50 \AA$ InAs/GaAs QW are calculated using the eight-band BF FTM and material parameters are adopted from Table 5-2. The obtained energies locating at the center of Brillouin zone $\left(k_{t}=0\right)$ and at the edge of Brillouin zone $\left(k_{t}=0.2\right)$ are then plotted versus the order of Fourier truncation $\left(N_{t r}\right)$ for different numbers of mesh points $(N)$ in Figure 5-3(a) and (b), respectively, showing that spurious solutions turn up earlier for greater $k_{t}$ in the $\mathrm{CB}$ while present only for nonzero $k_{t}$ in the VB. This observation agrees with the fact that spurious solutions are prone to present at large $k$ region. The plots also show that it 
is $N_{t r}$ that controls the occurrence of spurious solutions in FTM, not the step size of discretization as in FDM [62]. The cut-off method trades off the computation accuracy for controlling spurious solutions, but in practice sufficient accuracy has been achieved before spurious solutions take place. Therefore, the range for selecting $N_{t r}$ may be quite large. As a rule of thumb, a smaller $N_{t r}$ is demanded to resist spurious solutions for heterostructures with sharp interfaces, i.e., sharp geometry or drastic difference of material parameters.

Lassen et al. [61] split the set of Schrödinger Hamiltonian $\hat{H}$ in the kspace into two parts (the main part $\hat{H}_{n n^{\prime}}^{0}$ and the rest part $\hat{H}_{n n^{\prime}}^{1}$ ) using a unitary operator that block diagonalizes $\hat{H}$. They verified that spurious solutions can be controlled by adopting small $k$ (i.e. to cut off the large $k$ components) to ensure $\hat{H}_{n n^{\prime}}^{1}$ is much smaller than $\hat{H}_{n n^{\prime}}^{0}$. They also have stated that the failure to restrict the Fourier expansion coefficient $\mathbf{c}_{\mathbf{n}}$ in Eq. (5.12) to small $k$ components would lead to the appearance of spurious solutions. In FTM, hiring a large $N_{t r}$ may also cause the wild spread of Fourier expansion coefficient $\mathbf{c}_{\mathbf{n}}$, upon where the spurious solutions take place. Therefore, the Fourier expansion coefficients $\mathbf{c}_{\mathbf{n}}$ of the $\mathrm{CB}$ component for the solution of the electron ground state shown in Figure 5-3(a) are plotted in Figure 5-4 at $N_{t r}=40, N_{t r}=115$, and $N_{t r}=127$. The first Brillouin zone is marked within two dashed lines. The Fourier series $\mathbf{c}_{\mathbf{n}}$ of the envelope functions is confined well in the first Brillouin zone at $N_{t r}=40$, but falls out of the Brillouin zone since $N_{t r}=115$, which is exactly the point where the electron ground-state eigen energy starts to diverge in Figure 5-3(a). Hence, the wild-spreading spectrum of the Fourier expansion coefficient $\mathbf{c}_{\mathbf{n}}$ can be taken as the signature of spurious solutions. The divergent energy may also vibrate to be the same value as that associated with 
a smaller $N_{t r}$, therefore, it seems that energies at $\mathrm{N}_{\mathrm{tr}}=127$ in Figure 5-2 is not spurious solutions. However, the truth is exactly on the contrary.
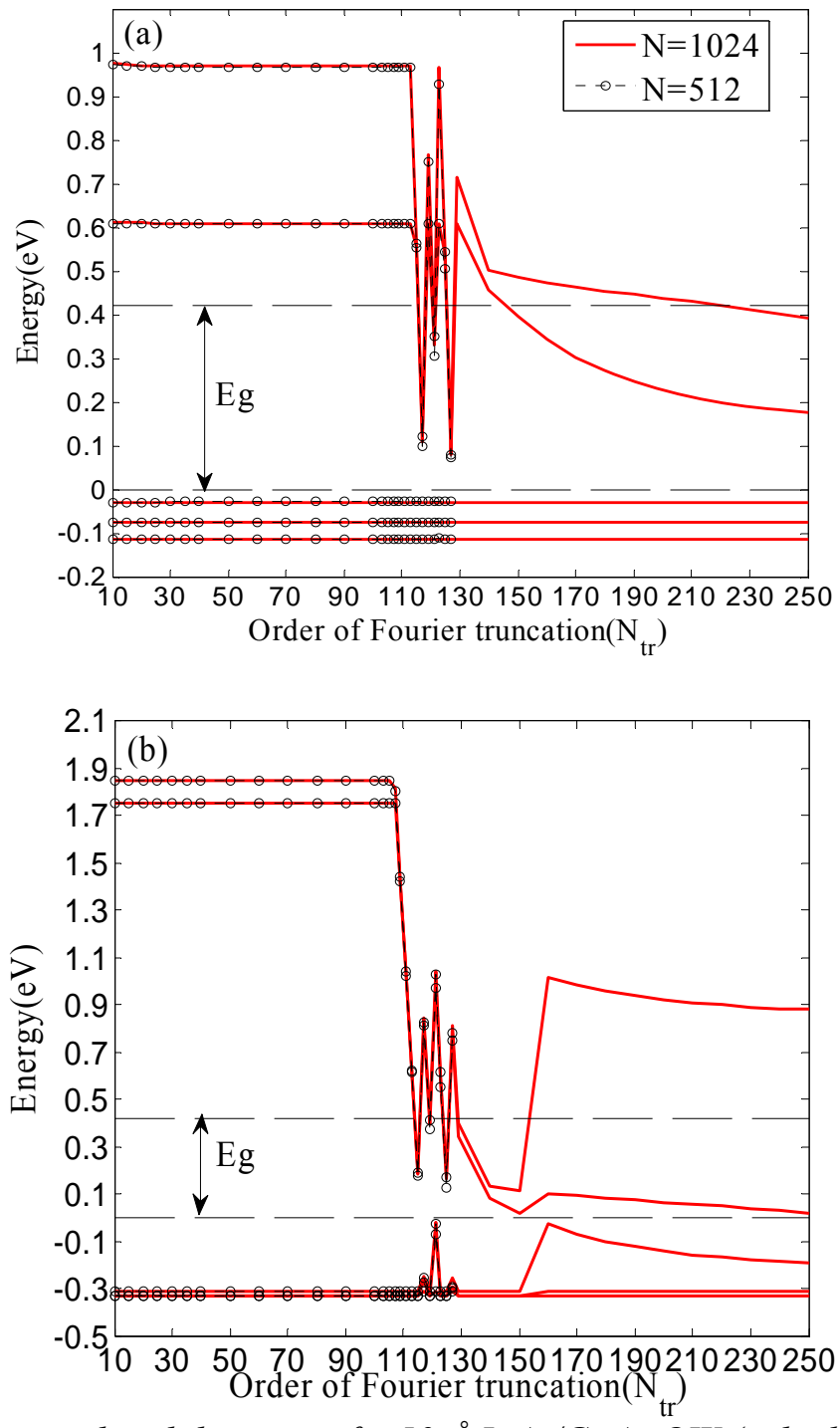

Figure 5-3 Energy band diagram of a 50 A InAs/GaAs $Q W$ (calculated with the BF operator ordering) varies with different orders of Fourier truncation $\left(N_{t r}\right)$ and different mesh points (N): (a) the band energies at the center of Brillouin Zone $\left(k_{t}=0\right)$, and (b) the band energiess at the edge of Brillouin Zone $\left(k_{t}=0.2\right)$. The two dashed lines indicated the forbidden band region. 

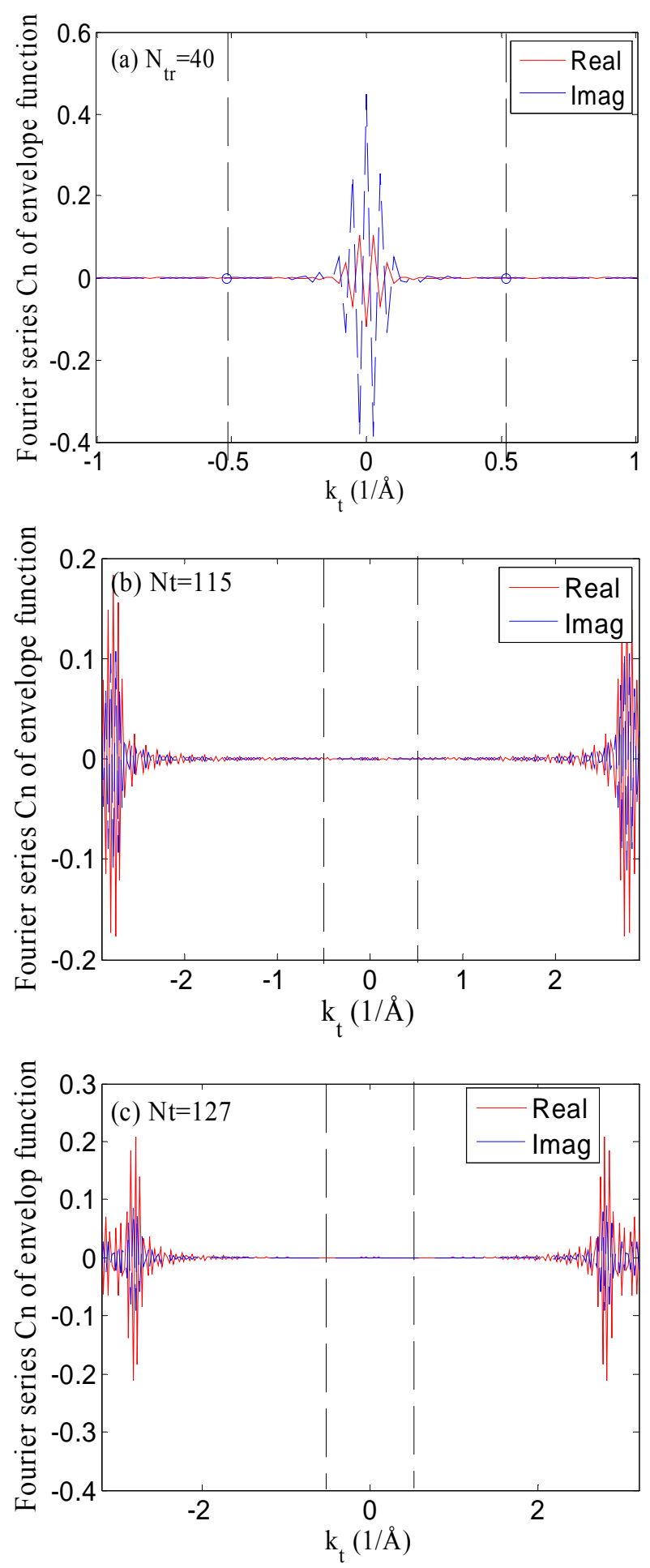

Figure 5-4 Fourier series $c_{n}$ of the envelope functions of a 50 A InAs/GaAs $Q W$ with the order of Fourier truncation (a) $N_{t r}=40$, (b) $N_{t r}=115$ and (c) $N_{t r}=127$. The computation is done with the BF operator ordering.The region composed by the two dashed lines indicates the Brillouin zone. 
This is further examined in the QWR case. The energy band structure of a wurtzite $18 \AA \times 18 \AA$ square GaN/AIN QWR is obtained by the six-band k.p calculation with both SYM and BF orderings (Hamiltonian matrices are in Appendix F). The obtained band structure is shown in Figure 5-5. Material parameters shown in Table 5-3 are used in the computation. In Figure 5-5, which is similar to Figure 5-1, the solutions particular to the BF ordering are very stable, while in the calculation with the SYM ordering, spurious solutions quickly turns up with increasing $k$ and appear even at $k_{t}=0$. Only at the very small $N_{t r}\left(N_{t r}=5\right)$, the energy dispersion shows similarity to that obtained by BF ordering in Figure 55(b), and can be considered as true solution, even though the accuracy is poor at a small truncation order. The major PDF components and the Fourier series $\mathbf{c}_{\mathbf{n}}$ of the envelop functions corresponding to the ground hole state are plotted in Figure 5-6 for $N_{t r}=10$ and at $N_{t r}=11$. The first Brillouin zones are marked within dashed box. Unlike that in Figure 5-6(a), major PDF component in Figure 5-6(b) locates at the corner of QWR, indicating this solution is non-physical. The Fourier series $\mathbf{c}_{\mathbf{n}}$ of the envelope functions at $N_{t r}=11$ shown in Figure 5-6(d) falls out of the first Brillouin zone. In contrast, the one corresponding to the solution with physical meaning in Figure 5-6(c) falls inside the first Brillouin zone. Recalling the case of QW computed with the BF FTM in Figure 5-4, it can be further confirmed that a large $N_{t r}$ may fail to confine the Fourier series $\mathbf{c}_{\mathbf{n}}$ of the envelope function in the small $k$-region and, thus, allow spurious solutions. This conclusion is true, no matter what kind of heterostructures and what kind of operator ordering. 

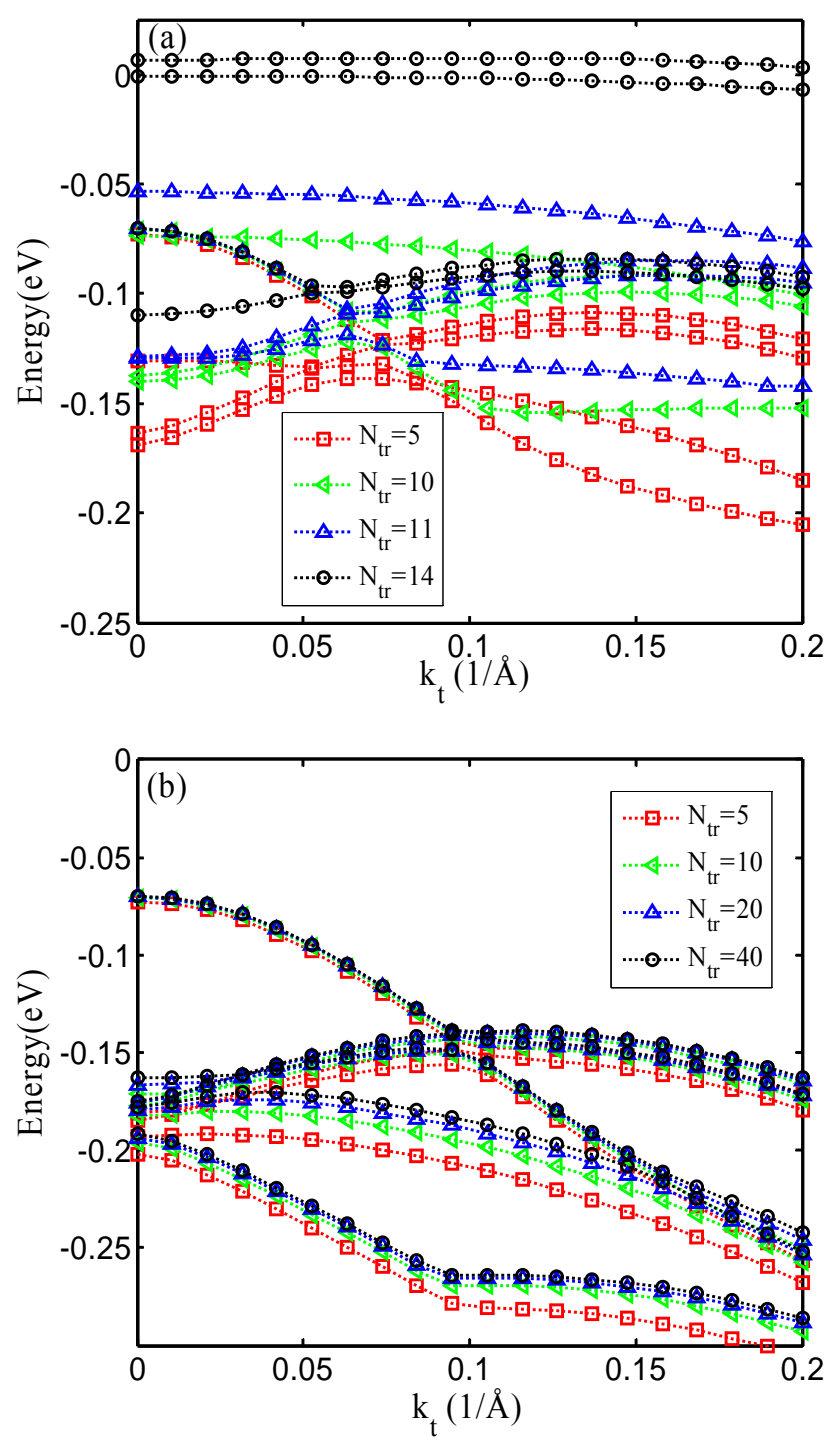

Figure 5-5 Energy band diagrams of a $18 \AA \times 18$ A square GaN/AlN QWR obtained using the six-band Hamiltonian matrix varies with various order of Fourier truncation $N_{t r}$ : (a) with the SYM operator ordering and (b) with the BF operator ordering. 
Table 5-3 Material parameters used to investigate spurious solutions in the GaN/AlN QWRs. All of the material parameters are after Ref. [200].

\begin{tabular}{lcc}
\hline \hline Parameter & GaN & AlN \\
Energy parameters $(\mathrm{eV})$ & & \\
$E_{g}$ at $300 \mathrm{~K}$ & 3.4376 & 6.1581 \\
$\Delta_{1}=\Delta_{c r}$ & 0.0100 & -0.1690 \\
$\Delta_{2}=\Delta_{3}=\Delta_{s o} / 3$ & 0.0057 & 0.0063 \\
Valance-band effective mass & & \\
$A_{1}$ & -7.21 & -3.86 \\
$A_{2}$ & -0.44 & -0.25 \\
$A_{3}$ & 6.68 & 3.58 \\
$A_{4}$ & -3.46 & -1.32 \\
$A_{5}$ & -3.40 & -1.47 \\
$A_{6}$ & -4.90 & -1.64 \\
\hline \hline
\end{tabular}

(a)

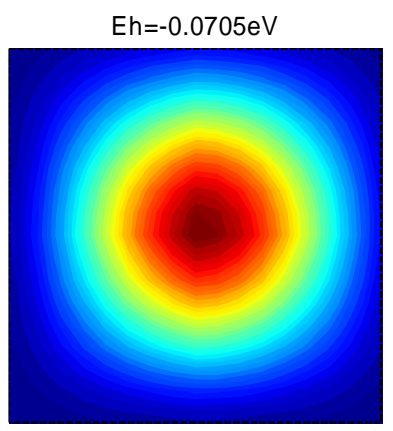

(c)

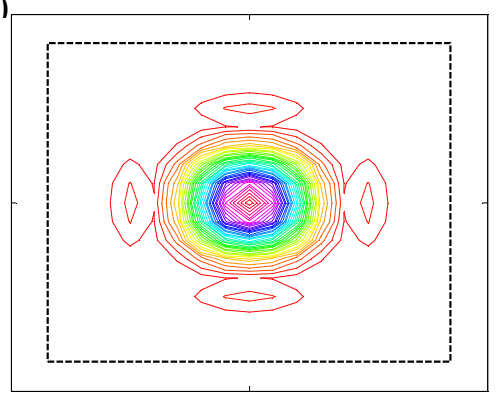

(b)

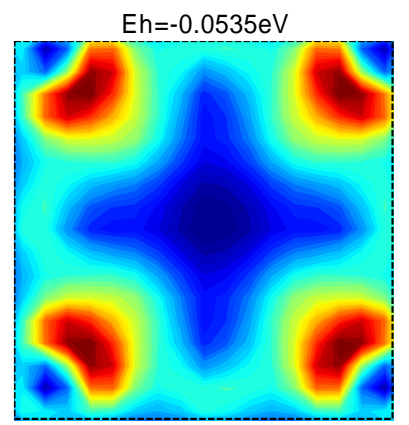

(d)

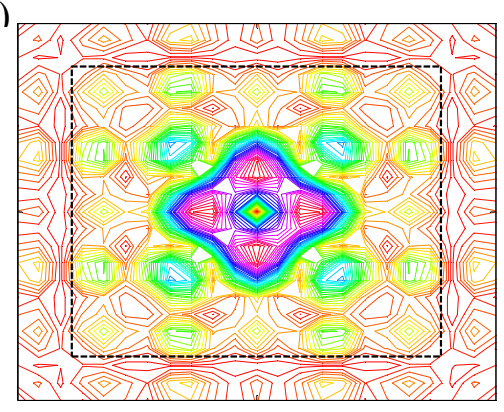

Figure 5-6 Major PDF components corresponding to the hole state of a $18 A \times 18$ A square GaN/AlN QWR with (a) $N_{t r}=10$ and (b) $N_{t r}=11$, and the corresponding Fourier series $\boldsymbol{c}_{\boldsymbol{n}}$ of the envelope function with (c) $N_{t r}=10$ and (d) $N_{t r}=11$. The computation is done with the SYM operator ordering. The dashed boxes in (c) and (d) indicated the first Brillouin zones. 


\subsection{Summary}

The BF operator ordering has been incorporated into FTM to boost the capability of tackling spurious solutions in the multiband $\mathrm{k} \cdot \mathrm{p}$ eigen problems of heterostructures. The new version of FTM collects the merits of both BF operator ordering and the inborn cut-off method in FTM, and is robust in tackling the problem of spurious solutions encountered by $\mathrm{k} \cdot \mathrm{p}$ calculation. It is verified that the situation of spurious solutions met by FTM is similar to that by FEM, except that FTM has easy and simple control of $N_{t r}$. Therefore, the origin of spurious solutions is in the $\mathrm{k} \cdot \mathrm{p}$ theory itself rather than numerical schemes. The BF operator ordering is capable enough of rejecting spurious solutions in the six-band calculation independently without considering the control of $N_{t r}$, but not in the eight-band computation, where spurious solutions exist in CB. Such incapability may be caused by the coupling of the $\mathrm{CB}$ and the VB. A proper Fourier truncation confines the Fourier series of the envelope functions within the first Brillouin zone. In practice, a proper order of Fourier truncation can be chosen for sufficiently good computation accuracy and competence to prevent spurious solutions.

Taking the advantage of the intrinsic cut-off characteristic, the Fourier transform technique can screen away spurious solutions without changing any material parameters and the consideration of specific boundary conditions. The determination of the cut-off point is much more convenient than that introduced by Lassen et al [61]. Therefore, the inborn cut-off method in FTM and the BF operator ordering complements each other nicely to ease the spurious solution situation met in the $\mathrm{k} \cdot \mathrm{p}$ calculation. This thesis illustrates that the BF operator ordering is indeed needed to resist spurious solutions in the six-band $\mathrm{k} \cdot \mathrm{p}$ 
computation, whereas the inborn cut-off in FTM is simple and effective to reject spurious solutions in the eight-band $\mathrm{k} \cdot \mathrm{p}$ computation. 


\section{CHAPTER 6}

\section{OPTICAL PROPERTIES OF QWS}

In this chapter, based on the electronic structures obtained from FTM, the calculation of the optical gain of III-Nitride QWs is illustrated. Next, the optical gain in InGaN/InAlGaN QWs with zero internal piezoelectric fields is then investigated neglecting many-body effects. Finally, the interband optical transition momentum matrix elements of zinc blende structures particular to Bahder's Hamiltonian are included finally.

\subsection{Application of FTM results on optical gain of III- Nitride QWs}

Chuang has detailed the calculation of electronic structures and the optical gain of GaN QWs in Ref. [182]. A block-diagonalized 6×6 Hamiltonian [106] (see Appendix F) composed by two $3 \times 3$ Hamiltonians was adopted to calculate the electronic structures of GaN QWs. The optical gain spectrum was then obtained with the spontaneous emission spectrum with Eq. (2.69) and Eq. (2.70). Although the significant internal field due to both the spontaneous and the strain induced piezoelectricity was neglected, the method in Ref. [182] is still adopted and further developed to apply FTM results in the optical gain computation for the purpose of comparison. All of the material parameters used in this section are tabulated in Table 6-1. 
Table 6-1 Material parameters used to compute optical properties of GaN/AlGaN QWs. All material parameters are after Ref.[182].

\begin{tabular}{|c|c|c|}
\hline $\begin{array}{l}\text { Parameter } \\
\end{array}$ & $\overline{\mathrm{GaN}}$ & AlN \\
\hline & 3.1892 & Lattice constants $(\AA)$ \\
\hline$c$ at $300 \mathrm{~K}$ & 5.1850 & 4.982 \\
\hline \multicolumn{3}{|l|}{ Energy parameters $(\mathrm{eV})$} \\
\hline$E_{g}$ at $300 \mathrm{~K}$ & 3.44 & 6.28 \\
\hline$\Delta_{I}=\Delta_{c r}$ & 0.016 & -0.0585 \\
\hline$\Delta_{2}=\Delta_{3}=\Delta_{s o} / 3$ & 0.004 & 0.0068 \\
\hline \multicolumn{3}{|c|}{ Conduction-band effective mass } \\
\hline$m_{e}^{t} / m_{0}$ & 0.18 & 0.25 \\
\hline$m_{e}^{z} / m_{0}$ & 0.20 & 0.33 \\
\hline \multicolumn{3}{|c|}{ Valance-band effective mass } \\
\hline$A_{1}$ & -6.56 & -3.95 \\
\hline$A_{2}$ & -0.91 & -0.27 \\
\hline$A_{3}$ & 5.65 & 3.68 \\
\hline$A_{4}$ & -2.83 & -1.84 \\
\hline$A_{5}$ & -3.13 & -1.95 \\
\hline$A_{6}$ & -4.86 & -2.91 \\
\hline \multicolumn{3}{|l|}{ Deformation potentials $(\mathrm{eV})$} \\
\hline$a_{c z}$ & -4.08 & \\
\hline$a_{c t}$ & -4.08 & \\
\hline$D_{1}$ & 0.7 & \\
\hline$D_{2}$ & 2.1 & \\
\hline$D_{3}$ & 1.4 & \\
\hline$D_{4}$ & -0.7 & \\
\hline \multicolumn{3}{|c|}{ Elastic stiffness constants (Gpa) } \\
\hline$C_{13}$ & 158 & 120 \\
\hline$C_{33}$ & 267 & 395 \\
\hline
\end{tabular}

\subsubsection{Interband transition matrix for III-Nitride QWs}

With the $6 \times 6$ Hamiltonian [106] (see Appendix F), the hole wavefunction in the III-Nitride QWs for the $m$ th valence subband can be rewritten as,

$$
\begin{aligned}
& \Psi_{m}^{U}\left(z ; k_{t}\right)=\frac{e^{i k_{t} \cdot r_{t}}}{\sqrt{A}}\left[g_{m}^{(1)}\left(z ; k_{t}\right)|1\rangle+g_{m}^{(2)}\left(z ; k_{t}\right)|2\rangle+g_{m}^{(3)}\left(z ; k_{t}\right)|3\rangle\right] \\
& \Psi_{m}^{L}\left(z ; k_{t}\right)=\frac{e^{i k_{t} \cdot r_{t}}}{\sqrt{A}}\left[g_{m}^{(4)}\left(z ; k_{t}\right)|4\rangle+g_{m}^{(5)}\left(z ; k_{t}\right)|5\rangle+g_{m}^{(6)}\left(z ; k_{t}\right)|6\rangle\right]
\end{aligned} .
$$


The band structure in CB is obtained with the single-band Hamiltonian matrix, and the wavefunction for the $n$th CB subband can be expressed as,

$$
\Psi_{n, k_{t}}^{c \eta}=\frac{e^{i k_{t} \cdot r_{t}}}{\sqrt{A}} \phi_{n}(z)|S, \eta\rangle
$$

where $\eta$ represents spin up or spin down.

Both $g_{m}^{(j)}\left(z ; k_{t}\right)$ and $\phi_{n}(z)$ can be expanded with Fourier series as,

$$
\begin{aligned}
& g_{m}^{(j)}\left(z ; k_{t}\right)=\sum_{N} c_{m}^{j, N} \exp \left\{i\left[k_{z}+N(2 \pi / L)\right] z\right\}, j=1,2, \cdots, 6, \\
& \phi_{n}(z)=\sum_{N} a_{n}^{N} \exp \left\{i\left[k_{z}+N(2 \pi / L)\right] z\right\}
\end{aligned}
$$

where $N$ is the order of Fourier truncation, $c_{m}^{i, N}$ and $a_{n}^{N}$ are envelope functions of VB and $\mathrm{CB}$, respectively, which can be obtained directly by solving the Hamiltonian matrix in the Fourier domain.

As being reviewed in Chapter 2 [Eq. (2.84)], the integration of the conjugate state of $\mathrm{CB}$ wavefunctions and the state of $\mathrm{VB}$ wavefunctions in the interband transition momentum matrix can be simplified as the integration of conjugated Fourier series of $\mathrm{CB}$ envelope function and Fourier series of $\mathrm{VB}$ envelope functions. Hence the interband transition momentum matrix elements in Ref. [182] can be rewritten as:

TE polarization $(\hat{e}=\hat{x}$ or $\hat{y} \perp$ c axis $)$ :

$$
\begin{aligned}
& \left|\left(M_{x}\right)_{n m}^{\sigma}\left(k_{t}\right)\right|^{2}=\frac{\left|\left\langle S\left|p_{x}\right| X\right\rangle\right|^{2}}{4}\left\{\left[\sum_{N}\left(a_{n}^{N}\right)^{*} c_{m}^{(1), N}\right]^{2}+\left[\sum_{N}\left(a_{n}^{N}\right)^{*} c_{m}^{(2), N}\right]^{2}\right\}, \quad \sigma=U \\
& \left|\left(M_{x}\right)_{n m}^{\sigma}\left(k_{t}\right)\right|^{2}=\frac{\left|\left\langle S\left|p_{x}\right| X\right\rangle\right|^{2}}{4}\left\{\left[\sum_{N}\left(a_{n}^{N}\right)^{*} c_{m}^{(4), N}\right]^{2}+\left[\sum_{N}\left(a_{n}^{N}\right)^{*} c_{m}^{(5), N}\right]^{2}\right\}, \quad \sigma=L
\end{aligned}
$$

TM polarization $(\hat{e}=\hat{z} \| c$ axis $)$ : 


$$
\begin{aligned}
\left|\left(M_{z}\right)_{n m}^{\sigma}\left(k_{t}\right)\right|^{2} & =\frac{\left|\left\langle S\left|p_{z}\right| Z\right\rangle\right|^{2}}{2}\left[\sum_{N}\left(a_{n}^{N}\right)^{*} c_{m}^{(3), N}\right]^{2}, \quad \sigma=U \\
& =\frac{\left|\left\langle S\left|p_{z}\right| Z\right\rangle\right|^{2}}{2}\left[\sum_{N}\left(a_{n}^{N}\right)^{*} c_{m}^{(6), N}\right]^{2}, \quad \sigma=L
\end{aligned}
$$

Since the upper and lower Hamiltonian matrices have the same VB structures [106, 182], Eq. (6.5) and Eq. (6.6) can be further simplified as,

TE polarization $(\hat{e}=\hat{x}$ or $\hat{y} \perp c$ axis $)$ :

$\left|\left(M_{x}\right)_{n m}^{\sigma}\left(k_{t}\right)\right|^{2}=\frac{\left|\left\langle S\left|p_{x}\right| X\right\rangle\right|^{2}}{4}\left\{\left[\sum_{N}\left(a_{n}^{N}\right)^{*} c_{m}^{(1), N}\right]^{2}+\left[\sum_{N}\left(a_{n}^{N}\right)^{*} c_{m}^{(2), N}\right]^{2}\right\}, \quad \sigma=U, L$.

TM polarization $(\hat{e}=\hat{z} \| c$ axis $)$ :

$$
\left|\left(M_{z}\right)_{n m}^{\sigma}\left(k_{t}\right)\right|^{2}=\frac{\left|\left\langle S\left|p_{z}\right| Z\right\rangle\right|^{2}}{2}\left[\sum_{N}\left(a_{n}^{N}\right)^{*} c_{m}^{(3), N}\right]^{2}, \quad \sigma=U, L
$$

\subsubsection{Optical gain of $\mathrm{GaN}-\mathrm{Al}_{0.3} \mathrm{Ga}_{0.7} \mathrm{~N} Q W \mathrm{~s}$}

Since the momentum matrix elements in Eq. (6.7) and Eq. (6.8) are obtained from the Fourier series of envelope functions, the spontaneous emission rate and the optical gain spectrum can be computed using Eq. (2.69) and Eq. (2.70), respectively. The integration cutoff point of $k_{\|}=0.1 \times 2 \pi / a$ ( $a$ is lattice constant), which is recommended by Eppenga [201], is selected in the calculations of the quasi-Fermi levels and the spontaneous emission rate. Figure 6-1 and Figure 6-2 show the spontaneous emission rate (on the right axis in arbitrary units) and the optical gain spectrum (on the left axis in the unit of $1 / \mathrm{cm}$ ) of a $26 \AA / 62 \AA$ $\mathrm{GaN} / \mathrm{Al}_{0.3} \mathrm{Ga}_{0.7} \mathrm{~N} \quad \mathrm{QW}$ and a $50 \AA / 50 \AA \quad \mathrm{GaN} / \mathrm{Al}_{0.3} \mathrm{Ga}_{0.7} \mathrm{~N} \quad \mathrm{QW}$, respectively. Chuang's calculation results [182] (hollow circles) are also numerically cited and reproduced in Figure 6-1 and Figure 6-2 for comparison. 


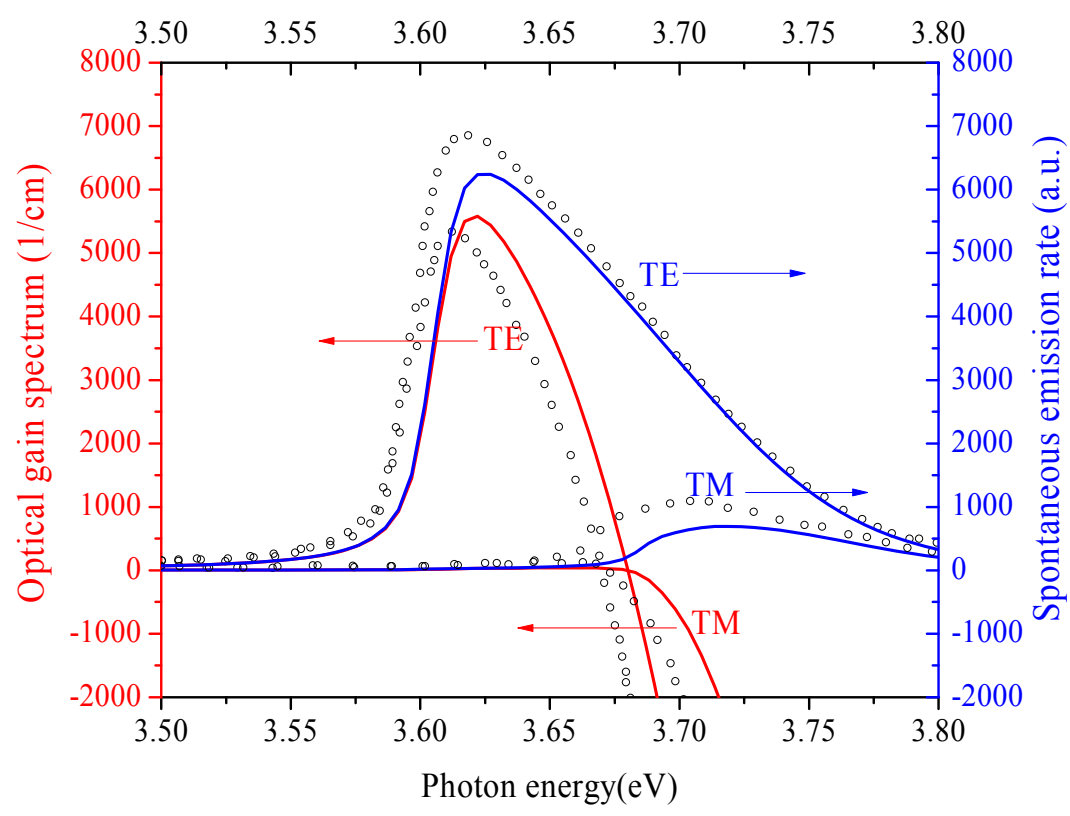

Figure 6-1 Spontaneous emission rate (on the right axis in arbitrary units) and optical gain spectrum (on the left axis in the unit of $1 / \mathrm{cm}$ ) of a $26 \AA / 62 \AA$ $\mathrm{GaN} / \mathrm{Al}_{0.3} \mathrm{Ga}_{0.7} \mathrm{~N} \mathrm{QW}$. The hollow circles denote the data numerically cited from Chuang's calculation.

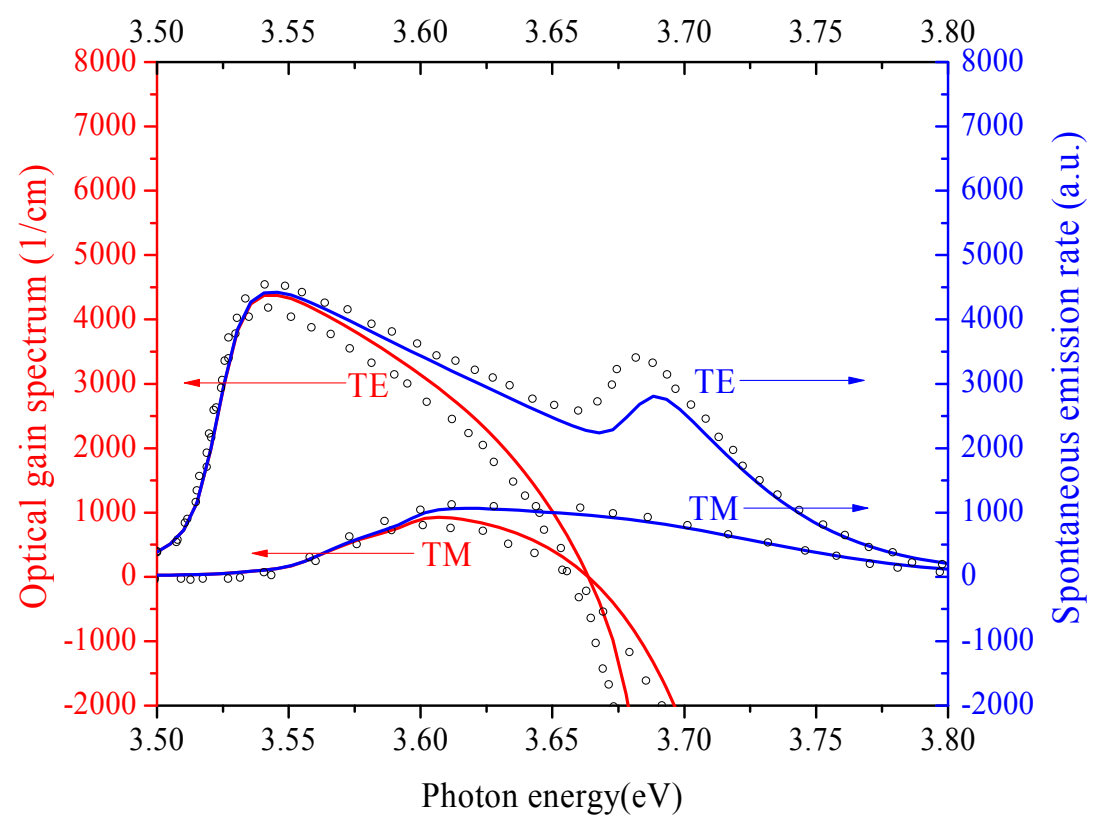

Figure 6-2 Spontaneous emission rate (on the right axis in arbitrary units) and optical gain spectrum (on the left axis in the unit of $1 / \mathrm{cm}$ ) of a $50 \AA / 50 \AA$ $G a N / A l_{0.3} G a_{0.7} N Q W$. The hollow circles denote the data numerically cited from Chuang's calculation. 
The significant features of the obtained results are: 1) sharper gain and spontaneous emission rate in Figure 6-1, 2) optical transition for the TE polarization is much more significant than the that for the TM polarization in two figures due to the compression strain, 3) larger separations between the optical gain and the spontaneous emission rate in Figure 6-1,4) one peak in the TE spontaneous emission rate shown in Figure 6-1, but two peaks in the TE spontaneous emission rate shown in Figure 6-2. The cited results also have these significant features. The small difference between the obtained results and the cited results lies at the different peak values of the optical gain and the spontaneous emission rate, and the different peak positions of the optical gain and the spontaneous emission rate. Such a small difference is reasonable since different numerical methods are involved. Therefore, it can be concluded that FTM indeed simplifies the computation of the interband transition matrix element, and thus the optical gain. The obtained reasonable optical gain spectrum further proofs the validity of the FTM approach.

\subsubsection{Optical gain of InGaN/InAlGaN QWs with a zero internal field}

The material parameters used in this section are tabulated in Table 6-2 and

Table 6-3. For the quaternary $\operatorname{In}_{\mathrm{x}} \mathrm{Al}_{\mathrm{y}} \mathrm{Ga}_{(1-\mathrm{x}-\mathrm{y})} \mathrm{N}$, the interpolation scheme is adopted as [202],

$$
G_{A B C D}^{\prime}=\frac{x y G_{A B D}^{\prime}(u)+y(1-x-y) G_{B C D}^{\prime}(v)+(1-x-y) x G_{A C D}^{\prime}(w)}{x y+y(1-x-y)+(1-x-y) x},
$$

Table 6-2 The binary material parameters used to interpolate $\mathrm{Al}_{x} \operatorname{In}_{y} G a_{z} N$. All of the material parameters are after Ref. [200].

\begin{tabular}{lccc}
\hline \hline \multicolumn{1}{c}{ Parameter } & GaN & AlN & InN \\
Lattice constants $(\AA)$ & & & \\
$a$ at $300 \mathrm{~K}$ & 3.189 & 3.112 & 3.545 \\
$c$ at $300 \mathrm{~K}$ & 5.185 & 4.982 & 5.703 \\
\hline \hline
\end{tabular}




\begin{tabular}{|c|c|c|c|}
\hline \multicolumn{4}{|l|}{$\begin{array}{l}\text { Energy } \\
\text { parameters }(\mathrm{eV})\end{array}$} \\
\hline$E_{g}$ at $300 \mathrm{~K}$ & 3.4376 & 6.1581 & 0.7561 \\
\hline$\Delta_{l}=\Delta_{c r}$ & 0.0100 & -0.1690 & 0.0400 \\
\hline$\Delta_{2}=\Delta_{3}=\Delta_{s o} / 3$ & 0.0057 & 0.0063 & 0.0017 \\
\hline \multicolumn{4}{|l|}{$\begin{array}{l}\text { Conduction-band } \\
\text { effective mass }\end{array}$} \\
\hline$m_{e}^{t} / m_{0}$ & 0.20 & 0.32 & 0.07 \\
\hline$m_{e}^{z} / m_{0}$ & 0.20 & 0.30 & 0.07 \\
\hline \multicolumn{4}{|l|}{$\begin{array}{l}\text { Valance-band } \\
\text { effective mass }\end{array}$} \\
\hline$A_{1}$ & -7.21 & -3.86 & -8.21 \\
\hline$A_{2}$ & -0.44 & -0.25 & -0.68 \\
\hline$A_{3}$ & 6.68 & 3.58 & 7.57 \\
\hline$A_{4}$ & -3.46 & -1.32 & -5.23 \\
\hline$A_{5}$ & -3.40 & -1.47 & -5.11 \\
\hline$A_{6}$ & -4.90 & -1.64 & -5.96 \\
\hline \multicolumn{4}{|l|}{$\begin{array}{l}\text { Deformation } \\
\text { potentials }(\mathrm{eV})\end{array}$} \\
\hline$a_{c z}$ & -4.9 & -3.4 & -3.5 \\
\hline$a_{c t}$ & -11.3 & -11.8 & -3.5 \\
\hline$D_{1}$ & -3.7 & -17.1 & -3.7 \\
\hline$D_{2}$ & 4.5 & 7.9 & 4.5 \\
\hline$D_{3}$ & 8.2 & 8.8 & 8.2 \\
\hline$D_{4}$ & -4.1 & -3.9 & -4.1 \\
\hline$D_{5}$ & -4.0 & -3.4 & -4.0 \\
\hline$D_{6}$ & -5.5 & -3.4 & -5.5 \\
\hline \multicolumn{4}{|l|}{$\begin{array}{l}\text { Elastic stiffness } \\
\text { constants (Gpa) }\end{array}$} \\
\hline$C_{11}$ & 390 & 396 & 223 \\
\hline$C_{12}$ & 145 & 137 & 115 \\
\hline$C_{13}$ & 106 & 108 & 92 \\
\hline$C_{33}$ & 398 & 373 & 224 \\
\hline \multicolumn{4}{|l|}{$\begin{array}{l}\text { Spontaneous } \\
\text { polarization }\left(\mathrm{C} / \mathrm{m}^{2}\right)\end{array}$} \\
\hline$P_{s p}$ & -0.034 & -0.090 & -0.042 \\
\hline $\begin{array}{l}\text { Piezoelectric } \\
\text { coefficients }(\mathrm{pm} / \mathrm{V})\end{array}$ & & & \\
\hline$d_{13}$ & -1.6 & -2.1 & -3.5 \\
\hline
\end{tabular}

* The linear interpolation formula $\mathrm{P}\left[\mathrm{A}_{\mathrm{x}} \mathrm{B}_{1-\mathrm{x}} \mathrm{N}=x \mathrm{P}(\mathrm{AN})+(1-x) \mathrm{P}(\mathrm{BN})\right]$ is used to for most of the parameters for $A_{x} B_{1-x} N$, except for the bandgap energy and the spontaneous polarization, which have bowing parameters shown in Table 6-3.

Table 6-3 The bowing parameters for the wurtzite ternary nitride compounds. All of the material parameters are after Ref. [200].

\begin{tabular}{lccc}
\hline \multicolumn{1}{c}{ Parameter } & GaInN & AlGaN & AlInN \\
Energy parameters $(\mathrm{eV})$ & & & \\
$E_{g}$ at $300 \mathrm{~K}$ & 1.4 & 0.7 & 2.5 \\
$\begin{array}{l}\text { Spontaneous polarization }\left(\mathrm{C} / \mathrm{m}^{2}\right) \\
P_{s p}\end{array}$ & -0.037 & -0.021 & -0.070 \\
\hline \hline
\end{tabular}

where $u=(1-x-y) / 2, v=(2-x-2 y) / 2$, and $w=(2-2 x-y) / 2$. The second order of piezoelectric polarization is not involved in the computation, since the 
bowing effect of AlInN was not recommended in Ref. [112]. The band offset ratio is $70: 30[85,203]$. To ensure that the internal piezoelectric field in $\mathrm{In}_{0.05} \mathrm{Ga}_{0.95} \mathrm{~N} / \mathrm{In}_{\mathrm{y}} \mathrm{Al}_{0.1} \mathrm{Ga}_{(0.9-\mathrm{y})} \mathrm{N} \quad \mathrm{QW}$ is zero (i.e., $P_{b}^{s p}+P_{b}^{\text {strain }}=P_{w}^{s p}+P_{b}^{\text {strain }}$ ), a composition of $\mathrm{y}=0.09$ is determined in this study, slightly smaller than the one (near $\mathrm{y}=0.1$ ) reported in Ref. [90].

Figure 6-3 presents the optical gain spectra for $\mathrm{In}_{0.05} \mathrm{Ga}_{0.85} \mathrm{~N} / \mathrm{Al}_{0.1} \mathrm{In}_{0.09} \mathrm{Ga}_{0.81} \mathrm{~N}$ QW with a well width of $35 \AA$ at carrier concentrations $\mathrm{n}=2 \times 10^{19}, 3 \times 10^{19}$, and $4 \times 10^{19} \mathrm{~cm}^{-3}$. Optical gain for TE polarization and TEM polarization are represented with solid lines and dashed lines, respectively. The predominance of TE polarization is due to the compressive strain in this QW structure. As the carrier concentration increases, the gain peak is slightly blueshifted due to the unobvious band filling effect. The band filling effect is the only cause for the carrier concentration-dependent peak shift [204] at low carrier concentration for which many body effect is negligible, i.e. free carrier approximation. The peak gain position in Figure 4(a) of Ref. [90] locates at $\sim 0.37$ $\mu \mathrm{m}(\sim 3.35 \mathrm{eV})$, which is obviously redshifted compared with the results shown in Figure 6-3. Therefore, the many body effects lead to the significant redshift in this QW structures with a zero internal field. This is in good agreement with the findings of Ref. [204].

To see the dependence of the gain peak on the well width, the optical gain peak (on the left axis) and the corresponding photon energy (on the right axis) versus well width of $\operatorname{In}_{0.05} \mathrm{Ga}_{0.85} \mathrm{~N} / \mathrm{Al}_{0.1} \mathrm{In}_{0.09} \mathrm{Ga}_{0.81} \mathrm{~N}$ QW is plotted in Figure 6-4. As the well width changes from $15 \AA$ to $50 \AA$, the peak of the gain increases significantly. After achieving a maximum value of around $33001 / \mathrm{cm}$ at well widths $L_{w}=35 \AA$, it decreases slowly. The VB band energies associated with 
$L_{w}=15 \AA$ and $L_{w}=35 \AA$ are drawn in Figure 6-5(a) and Figure 6-5(b), respectively.

It can be seen that the energy separation of subbands in Figure 6-5(b) is more obvious than that in Figure 6-5(a), which may be the reason of a higher optical peak gain arises at $L_{w}=35 \AA$ in Figure 6-4. In addition, photon energies at the peak gain vary from $3.463 \mathrm{eV}$ to $3.522 \mathrm{eV}$ (i.e. $\sim 60 \AA$ redshift) as the well width changing from $15 \AA$ to $50 \AA$. This can be ascribed to the redshift in larger quantum structures.

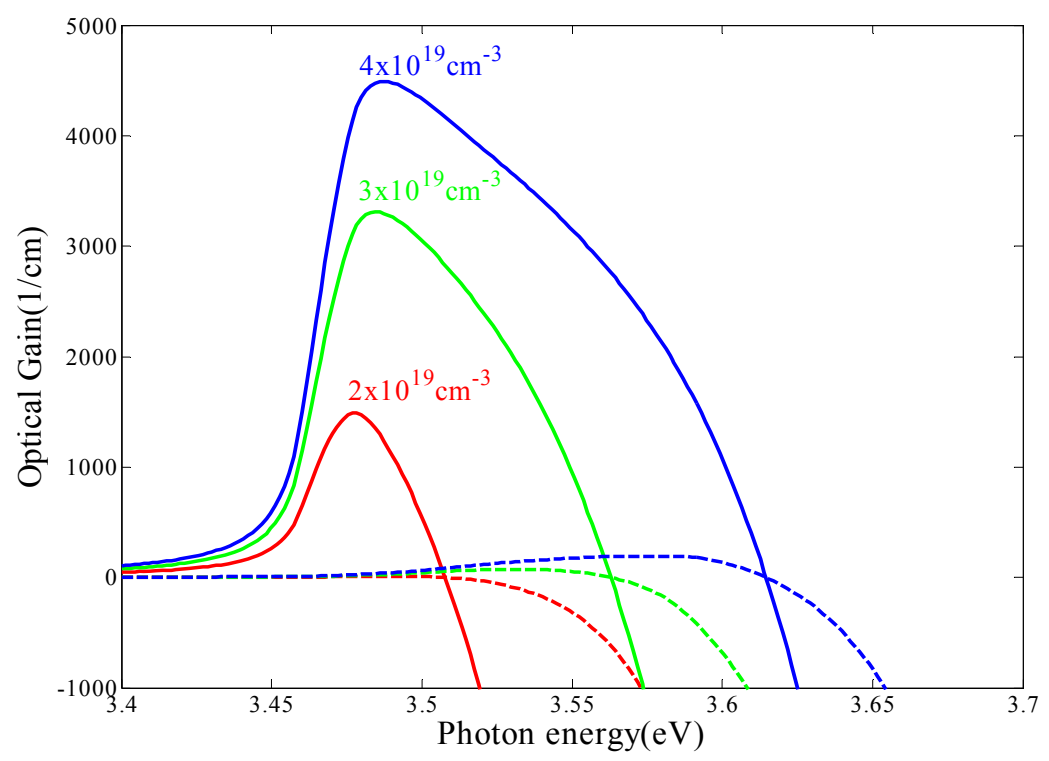

Figure 6-3 Optical gain spectra for the $\operatorname{In}_{0.05} \mathrm{Ga}_{0.85} \mathrm{~N} / \mathrm{Al}_{0.1} \mathrm{In}_{0.09} \mathrm{G} a_{0.81} \mathrm{~N} \mathrm{QW}$ with a well width of 35 A at the carrier concentrations $n=2 \times 10^{19}, 3 \times 10^{19}$, and $4 \times 10^{19} \mathrm{~cm}^{-3}$. Solid lines are for the TE polarization. Dashed lines are for the TM polarization. 


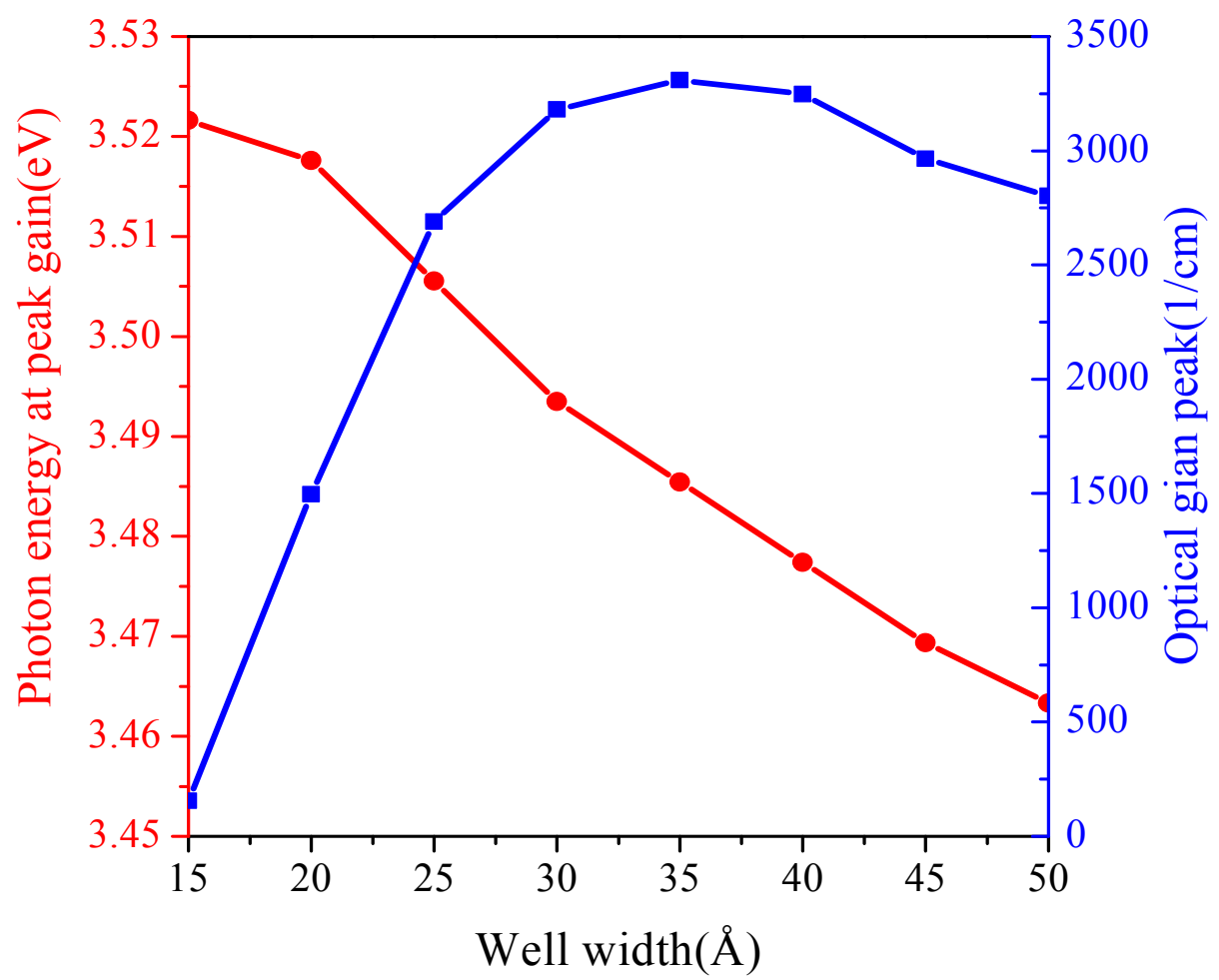

Figure 6-4 Optical peak gain as a function of the well width (on the right axis in the unit of $1 / \mathrm{cm}$ ), and the corresponding position of photon energy (on the left axis in the unit of $\mathrm{eV}$ ) of a $\mathrm{In}_{0.05} \mathrm{G} a_{0.85} \mathrm{~N} / \mathrm{Al}_{0.1} \mathrm{I} n_{0.09} \mathrm{G} a_{0.81} \mathrm{~N} \mathrm{QW}$. 

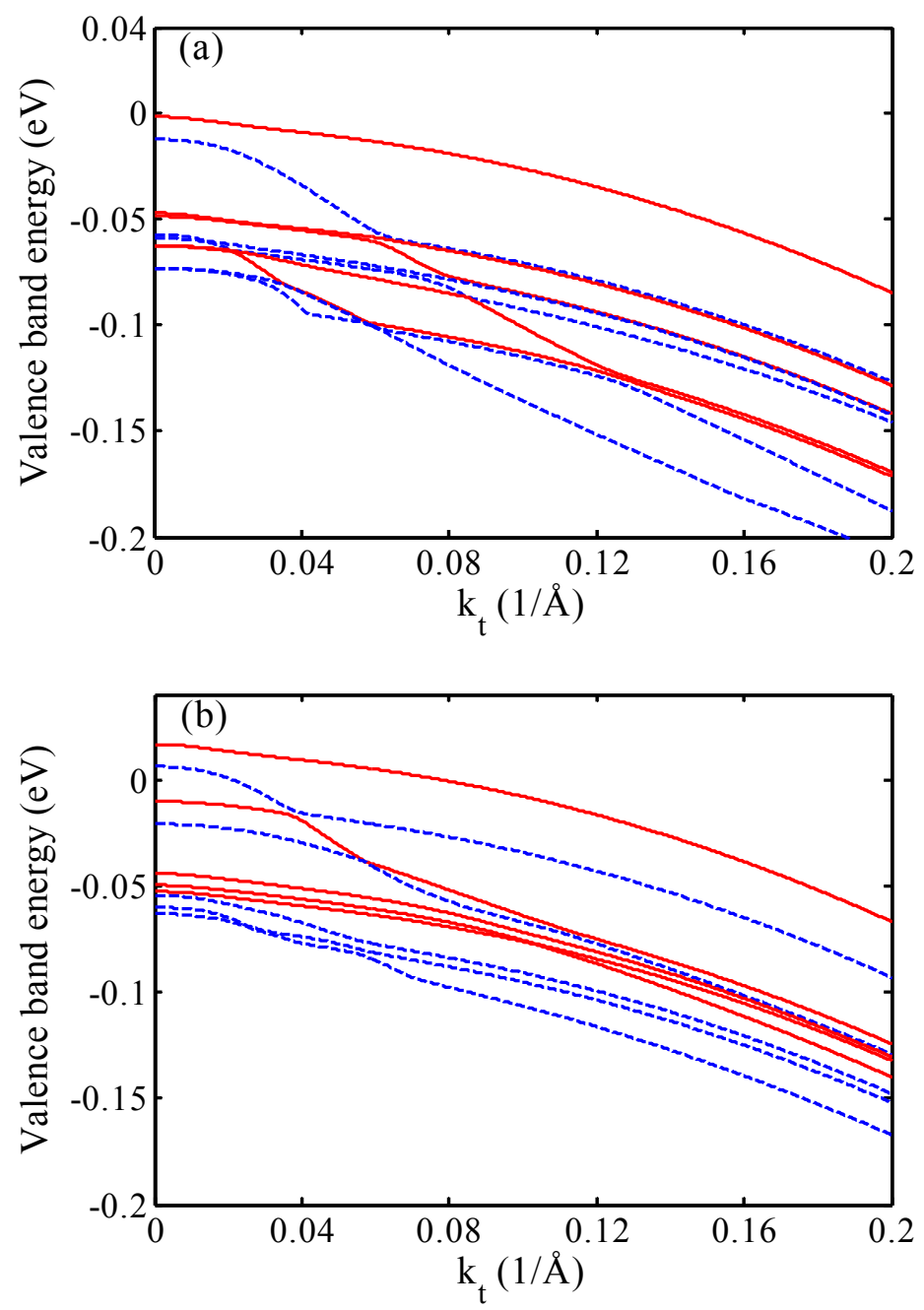

Figure 6-5 VB band energy of the $\operatorname{In}_{0.05} \mathrm{Ga}_{0.85} \mathrm{~N} / \mathrm{Al}_{0.1} \mathrm{In}_{0.09} \mathrm{Ga} a_{0.81} \mathrm{~N} \mathrm{QWs}$ with two different well widths: (a) $L_{w}=15 \AA$, and (b) $L_{w}=35 \AA$. The solid lines represent the HH state and the dashed lines describe the LH state.

\subsection{Interband transition matrix elements for zinc blende \\ QWs}

The Bahder's Hamiltonian $[107,153,154]$ (see Appendix F) is adopted to investigate the electronic structures of zinc blende heterostructures. With the FTM results, the associated interband momentum transition matrix elements are as follows, 


$$
\begin{aligned}
& M_{c, v}^{x, \downarrow}=\left\langle\psi_{c}\left|p_{x}\right| \psi_{v}\right\rangle \\
& =\sum_{n} \sum_{j_{c}=1,3,5,8} \sum_{j_{v}=1,3,5,8}\left[\left(c_{j_{c}}^{c, n}\right)^{*} \cdot c_{j_{v}}^{v, n}\right]\left\langle u_{j_{c}}\left|p_{x}\right| u_{j_{v}}\right\rangle\left(j_{c} \neq j_{v}\right) \\
& =P_{0} \sum_{n}\left(c_{1}^{c, n}\right)^{*}\left\{\frac{1}{\sqrt{6}} c_{3}^{v, n}+\frac{1}{\sqrt{2}} c_{5}^{v, n}+\frac{1}{\sqrt{3}} c_{8}^{v, n}\right\} \\
& -P_{0} \sum_{n} c_{1}^{v, n}\left\{\frac{1}{\sqrt{6}}\left(c_{3}^{c, n}\right)^{*}+\frac{1}{\sqrt{2}}\left(c_{5}^{c, n}\right)^{*}+\frac{1}{\sqrt{3}}\left(c_{8}^{c, n}\right)^{*}\right\} \\
& M_{c, v}^{x, \uparrow}=\sum_{n} \sum_{j_{c}=2,4,6,7} \sum_{j_{v}=2,4,6,7}\left[\left(c_{j_{c}}^{c, n}\right)^{*} \cdot c_{j_{v}}^{v, n}\right]\left\langle u_{j_{c}}\left|p_{x}\right| u_{j_{v}}\right\rangle\left(j_{c} \neq j_{v}\right) \\
& =P_{0} \sum_{n}\left(c_{2}^{c, n}\right)^{*}\left\{-\frac{1}{\sqrt{2}} c_{4}^{v, n}-\frac{1}{\sqrt{6}} c_{6}^{v, n}+\frac{1}{\sqrt{3}} c_{7}^{v, n}\right\} \\
& +P_{0} \sum_{n} c_{2}^{v, n}\left\{\frac{1}{\sqrt{2}}\left(c_{4}^{c, n}\right)^{*}+\frac{1}{\sqrt{6}}\left(c_{6}^{c, n}\right)^{*}-\frac{1}{\sqrt{3}}\left(c_{7}^{c, n}\right)^{*}\right\} \\
& M_{c, v}^{y, \downarrow}=\left\langle\psi_{c}\left|p_{y}\right| \psi_{v}\right\rangle \\
& =\sum_{n} \sum_{j_{c}=1,3,5,8} \sum_{j_{v}=1,3,5,8}\left[\left(c_{j_{c}}^{c, n}\right)^{*} \cdot c_{j_{v}}^{v, n}\right]\left\langle u_{j_{c}}\left|p_{y}\right| u_{j_{v}}\right\rangle\left(j_{c} \neq j_{v}\right) \\
& =i P_{0} \sum_{n}\left(c_{1}^{c, n}\right)^{*}\left\{\frac{1}{\sqrt{6}} c_{3}^{v, n}-\frac{1}{\sqrt{2}} c_{5}^{v, n}+\frac{1}{\sqrt{3}} c_{8}^{v, n}\right\} \\
& +i P_{0} \sum_{n} c_{1}^{v, n}\left\{\frac{1}{\sqrt{6}}\left(c_{3}^{c, n}\right)^{*}-\frac{1}{\sqrt{2}}\left(c_{5}^{c, n}\right)^{*}+\frac{1}{\sqrt{3}}\left(c_{8}^{c, n}\right)^{*}\right\} \\
& M_{c, v}^{y, \uparrow}=\left\langle\psi_{c}\left|p_{y}\right| \psi_{v}\right\rangle \\
& =\sum_{n} \sum_{j_{c}=2,4,6,7} \sum_{j_{v}=2,4,6,7}\left[\left(c_{j_{c}}^{c, n}\right)^{*} \cdot c_{j_{v}}^{v, n}\right]\left\langle u_{j_{c}}\left|p_{y}\right| u_{j_{v}}\right\rangle\left(j_{c} \neq j_{v}\right) \\
& =i P_{0} \sum_{n}\left(c_{2}^{c, n}\right)^{*}\left\{-\frac{1}{\sqrt{2}} c_{4}^{v, n}+\frac{1}{\sqrt{6}} c_{6}^{v, n}-\frac{1}{\sqrt{3}} c_{7}^{v, n}\right\} \\
& +i P_{0} \sum_{n} c_{2}^{v, n}\left\{-\frac{1}{\sqrt{2}}\left(c_{4}^{c, n}\right)^{*}+\frac{1}{\sqrt{6}}\left(c_{6}^{c, n}\right)^{*}-\frac{1}{\sqrt{3}}\left(c_{7}^{c, n}\right)^{*}\right\} \\
& M_{c, v}^{z, \downarrow}=\left\langle\psi_{c}\left|p_{z}\right| \psi_{v}\right\rangle \\
& =\sum_{n} \sum_{j_{c}=1,6,7} \sum_{j_{v}=1,6,7}\left[\left(c_{j_{c}}^{c, n}\right)^{*} \cdot c_{j_{v}}^{v, n}\right]\left\langle u_{j_{c}}\left|p_{z}\right| u_{j_{v}}\right\rangle\left(j_{c} \neq j_{v}\right) \\
& =i P_{0} \sum_{n}\left(c_{1}^{c, n}\right)^{*}\left\{-\frac{2}{\sqrt{6}} c_{6}^{v, n}-\frac{1}{\sqrt{3}} c_{7}^{v, n}\right\} \\
& +i P_{0} \sum_{n} c_{1}^{v, n}\left\{\frac{2}{\sqrt{6}}\left(c_{6}^{c, n}\right)^{*}+\frac{1}{\sqrt{3}}\left(c_{7}^{c, n}\right)^{*}\right\}
\end{aligned}
$$




$$
\begin{aligned}
M_{c, v}^{z, \uparrow} & =\left\langle\psi_{c}\left|p_{z}\right| \psi_{v}\right\rangle \\
& =\sum_{n} \sum_{j_{c}=2,3,8} \sum_{j_{v}=2,3,8}\left[\left(c_{j_{c}}^{c, n}\right)^{*} \cdot c_{j_{v}}^{v, n}\right]\left\langle u_{j_{c}}\left|p_{z}\right| u_{j_{v}}\right\rangle\left(j_{c} \neq j_{v}\right) \\
& =i P_{0} \sum_{n}\left(c_{2}^{c, n}\right)^{*}\left\{-\frac{2}{\sqrt{6}} c_{3}^{v, n}+\frac{1}{\sqrt{3}} c_{8}^{v, n}\right\} \\
& +i P_{0} \sum_{n} c_{2}^{v, n}\left\{\frac{2}{\sqrt{6}}\left(c_{3}^{c, n}\right)^{*}-\frac{1}{\sqrt{3}}\left(c_{8}^{c, n}\right)^{*}\right\}
\end{aligned}
$$

where $P_{0}=-i\left\langle S\left|p_{x}\right| X\right\rangle=-i\left\langle S\left|p_{y}\right| Y\right\rangle=-i\left\langle S\left|p_{z}\right| Z\right\rangle$. As the Fourier series of the envelope function are determined by solving the Hamiltonian matrix in the momentum space, the values of Eq. (6.10) to Eq. (6.15) can be calculated very easily, and so can the optical gain.

\subsection{Summary}

With the aid of the Fourier expansion of envelope function in FTM and the orthogonally of the exponential function, the interband transition matrix elements can be obtained by integrating the Fourier series of envelope function other than the wavefunctions. This simplifies the computation of the optical gain dramatically. The interband transition matrix elements for Chuang's $3 \times 3$ Hamiltonian matrix (Appendix F) and Bahder's $8 \times 8$ Hamiltonian matrix (Appendix F) have been listed for reference.

The optical gain of $\mathrm{GaN}-\mathrm{Al}_{0.3} \mathrm{Ga}_{0.7} \mathrm{~N}$ QWs has been calculated successfully using the FTM results (band energies and the corresponding Fourier series of envelope functions). The agreement of the calculated optical gain with the reported results further verifies the validity of the FTM computation.

An $\mathrm{In}_{0.05} \mathrm{Ga}_{0.85} \mathrm{~N} / \mathrm{Al}_{0.1} \mathrm{In}_{0.09} \mathrm{Ga}_{0.81} \mathrm{~N}$ QW structure with a zero internal field has been proposed using Vurgaftman's parameters [200]. The optical properties of this structure under lower carrier concentration have been investigated. Blueshift 
arises with increasing carrier concentration due to band filling, provided that the many body effects are still not big enough. $35 \AA \operatorname{In}_{0.05} \mathrm{Ga}_{0.85} \mathrm{~N} / \mathrm{Al}_{0.1} \mathrm{In}_{0.09} \mathrm{Ga}_{0.81} \mathrm{~N}$ QW has the highest peak gain because of its bigger energy separation between different states. 


\section{CHAPTER 7}

\section{CONCLUSIONS AND RECOMMENDATIONS}

In this chapter, the major conclusions in this study are summarized and the recommendations for future works are given as well.

\subsection{Conclusions}

This main theme of this thesis is to explore FTM toward investigating the electronic structures and the optical properties of low dimensional heterostructures. The major contributions of this thesis include the following: 1) the formulation of a meshless FTM and the suggestions of critical factors to control computation accuracy; 2) understanding the dimension effect, the strain effect, and the wetting layer effect on the electronics structures of truncated pyramidal QDs; 3) the removal of spurious solutions taking advantages of the intrinsic cut-off characteristic of FTM and the BF operator ordering; 4) applying FTM results to investigate the optical properties of QWs.

The meshless FTM has been presented as an approach to solving the 3D multiband $\mathrm{k} \cdot \mathrm{p}$ eigen problems. Compared with other developed numerical techniques, the meshless FTM has the following merits: 1) Fourier transform does not involve a troublesome differential or a integration process; 2) an awkward meshing process can be escaped tactfully via introducing the analytical Fourier transform of QD geometric shape directly, 3) FTM also has great flexibility to adapt to various QD shapes, structures (superlattices or isolated structures), and semiconductor material systems, 4) the most interesting feature is that spurious 
solutions can be resisted by truncating high-orders of Fourier frequencies, 5) FTM also brings great convenience for the optical gain computation. Furthermore, the meshless FTM for 3D-confined QDs is also applicable to 2D-confined and 1Dconfined (QWs and QWRs) due to its generality.

The electronic structures of isolated QDs have been calculated successfully with meshless FTM. The critical factors of the order of Fourier truncation and the periodic length that determine computation accuracy have also been discussed elaborately. As a rule of thumb, the periodic length in the computation for isolated QDs is recommended to be three times the largest dimension of the QD structure, $N_{t r x}=N_{t r y}=N_{t r z}=7$ is chosen as the order of Fourier truncation for the pyramidal examples, and a lower order truncated Fourier harmonics is applicable to smooth structures. Apart from truncating higher Fourier harmonics, the computation volume can be further reduced by eliminating the very small components in the momentum Hamiltonian matrix.

After exploring the meshless FTM, this thesis has investigated the dimension effect, the strain effect, and the wetting layer effect on the electronic structures of a series of truncated pyramidal QDs, as well as the redshift in $\operatorname{InAs} / \operatorname{In}_{\mathrm{x}} \mathrm{Ga}_{(1-\mathrm{x})} \mathrm{As}$ QDs. They are listed below:

1). The ground-state energy variation for a series of truncated pyramidal QDs shows that the electron state is the most sensitive to the dimension effect and the strain effect because of the small effective mass of an electron. The PDF study on a non-truncated pyramidal QD and a half-truncated pyramidal QDs shows that the strain effect is exhibited in the aspects of reduction of band mixing, the variations of the profile and the location of PDFs, and the weaker confinement to carriers. These facts are ascribed to 
the change in potential wells caused by the strain effect. Because of the large effective mass, the major component of a hole's state are more adapted to the fluctuation due to strain than the fluctuation due to strain in the major components of an electron's state. The strain effect on the ground-state energies and the PDFs is more prominent for sharper QD due to strain concentration.

2). The influence of the wetting layer on the ground-state electron energy can be as large as $0.05 \mathrm{eV}$ in very flat truncated pyramidal QDs. The wetting layer does not change the position of the band edge in the dot region very much. However, it widens the potential well, and thus the reduction of the energy band gap. The visual contours of PDFs illustrate that the wetting layer drives a small portion of the PDF of E1 from barrier region into the wetting layer but pushes a small portion of PDF of HH1 from the dot region into the wetting layer.

3). Redshift of the ground-state transitions between $\mathrm{CB}$ and VBs in InAs/ $\operatorname{In}_{\mathrm{x}} \mathrm{Ga}_{(1-\mathrm{x})} \mathrm{As}$ QDs can be realized by increasing the In composition to reduce the strain effect, and thus the shrinkage of the band gap. The consistency between the band-edge profiles and the ground-state energies demonstrates that the effect of strain on redshift becomes less remarkable as increasing the In component. Thicker LCL can be used to weaken thermal quenching due to the increase of In composition. The most proper range of In composition for redshift is $0 \leq x \leq 0.33$ to avoid size fluctuations and a small band discontinuity.

Subsequently, BF operator ordering has been incorporated to formulate a new version of FTM toward settling spurious solutions problem in the multiband 
$\mathrm{k} \cdot \mathrm{p}$ eigen problems of heterostructures. The new version of FTM gathers the merits of both the BF operator ordering and the inborn cut-off method in FTM, and, therefore, is robust in eliminating spurious solutions. The BF operator ordering is capable enough of rejecting spurious solutions in the six-band calculation independently without any special control of $N_{t r}$. The incapability of BF operator ordering in resisting the spurious solutions in the eight-band computation for QW may be caused by the coupling of CB and VB, as well as the small influence of the $\mathrm{BF}$ operator ordering on $\mathrm{CB}$ for the $1 \mathrm{D}$ computation. This incapability of $\mathrm{BF}$ operator ordering can be remedied by the intrinsic cut-off in FTM without changing any material parameters and without the consideration of specific boundary conditions.

Finally, the FTM results have been applied to calculate the optical properties of III-Nitride QWs successfully. Thus, the validity of FTM results is further verified. The integration of wavefunctions in the interband transition matrix elements is transferred to the integration of the Fourier series of envelope functions with the orthogonality of exponential functions, thus providing a great convenience to the computation process. Interband transition matrix elements particular to the eight-band computation and the six-band computation have been provided. An $\operatorname{In}_{0.05} \mathrm{Ga}_{0.85} \mathrm{~N} / \mathrm{Al}_{0.1} \mathrm{In}_{0.09} \mathrm{Ga}_{0.81} \mathrm{~N}$ QW structure has been proposed to ensure a zero internal piezoelectric field. The optical properties of this structure under lower carrier concentration have been investigated. Blueshift arises with the increase of carrier concentration due to band filling (provided that the many body effects are still not big enough). The $\operatorname{In}_{0.05} \mathrm{Ga}_{0.85} \mathrm{~N} / \mathrm{Al}_{0.1} \mathrm{In}_{0.09} \mathrm{Ga}_{0.81} \mathrm{~N} \mathrm{QW}$ with a well width of $35 \AA$ has the highest peak gain because of its slightly remarkable subbands separation. 
In general, FTM formulated in this thesis is expected to be useful in investigating the electronic structures of semiconductor heterostructures beyond the scope of this study. The nature of FTM itself opens avenues to a host of other materials (e.g. $\mathrm{Si}$ structures, $\mathrm{ZnO}$ nanotube, $\mathrm{GaN}$ nanowire, etc.). Other achievements in this thesis also provide a good guideline for the experimental application of low dimensional heterostructures.

\subsection{Recommendations}

Despite the full stretch has been put into this thesis, some works could be improved and further investigated. Four possible directions for future research are: 1) further reducing the computation amount, 2) incorporating the effects of the piezoelectric field and the substrate orientation, 3) further investigating the optical properties of heterostructures, and 4) solving the Schrödinger-Poisson equations.

1). Further reducing the computation amount. The size of Hamiltonian matrix has been reduced dramatically by truncating the high-orders of harmonics. However, a high percent of nonzero elements (up to $80 \%$ in the eight-band QD computation) makes the solving of eigen equation very timeconsuming. To economize the computation time and computer memory further, a Hamiltonian matrix in the real space after block-diagonalization [160] or symmetry operation [40] can be adopted in the QD computation.

2). Incorporating the effects of the piezoelectric field and the substrate orientation. The FTM computation can be improved further by adding the effects of the piezoelectric field and the substrate orientation. The piezoelectric field is one of the main factors affecting the photoluminescence efficiency of light emission devices, especially for the 
III-nitride heterostructures. It would be useful to take this effect into account and explore effective ways for suppressing the droop effect [205207] in the optoelectronic devices. In addition, since heterostructrues are increasingly grown on high index surfaces to improve the performance of optoelectronic devices [172, 208, 209], it is necessary to add the effect of substrate orientation to cater to this trend.

3). Further investigating the optical properties of heterostructures. With the given interband transition matrix elements, an investigation of the optical properties of QWRs and QDs must be done in the future. Another major concern would be involving the many body effect in the calculation.

4). Solving the Schrödinger-Poisson equations. In practice devices, the doping concentration is usually high, and thus there is a potential distortion. The Schrödinger-Poisson equations [210-212] can provide valuable information for the design of optoelectronic devices. Consequently, further studies should focus on solving the Schrödinger-Poisson equation. 


\section{APPENDICES}

\section{A. Kane model}

Kane model gives a basic but useful illustration of group III-V direct band materials for the lowest CB with the S-like unit cell wavefunctions and highest valence bands (VBs) made from the P-like unit cell wavefunction near the center of Brillouin zone. It adds the perturbation approximation, but misses an accuracy description of the $\mathrm{HH}$ band. The second-order four-band Kane Hamiltonian for zinc blende is,

$$
H_{\text {int }}=\frac{\hbar^{2} k^{2}}{2 m_{0}}+\left(\begin{array}{cccc}
s & x & y & z \\
E_{c}+A^{\prime} k^{2} & i P k_{x} & i P k_{y} & i P k_{z} \\
-i P k_{x} & E_{v}+L^{\prime} k_{x}^{2} & N^{\prime} k_{x} k_{y} & N^{\prime} k_{x} k_{z} \\
& +M\left(k_{y}^{2}+k_{z}^{2}\right) & & \\
-i P k_{y} & N^{\prime} k_{x} k_{y} & E_{v}+L^{\prime} k_{y}^{2} & N^{\prime} k_{y} k_{z} \\
& & +M\left(k_{x}^{2}+k_{z}^{2}\right) & \\
-i P k_{z} & N^{\prime} k_{x} k_{z} & N^{\prime} k_{y} k_{z} & E_{v}+L^{\prime} k_{z}^{2} \\
& & & +M\left(k_{x}^{2}+k_{y}^{2}\right)
\end{array}\right) \text {, }
$$

where,

$$
\begin{aligned}
& L^{\prime}=F^{\prime}+2 G \\
& M=H_{1}+H_{2} \\
& N^{\prime}=F^{\prime}-G+H_{1}-H_{2}
\end{aligned}
$$

$E_{c}$ and $E_{v}$ are the band-edge of $\mathrm{CB}$ and $\mathrm{VB}$, respectively. $P$ is the momentum matrix element. $A^{\prime}$ is the effective mass parameter of $\mathrm{CB}$, and other Kan parameters, i.e., $L^{\prime}, M, N^{\prime}, F^{\prime}, G, H_{1}$, and $H_{2}$, are nearly identical to those of Dresselhaus, Kip, and Kittle [46, 148]. The so-called spin-orbit interaction between 
the spin of an electron and the orbital angular momentum of the $p$-states are taken account as follows,

$$
\mathrm{H} u_{n, \mathbf{k}}(\mathbf{r})=\left[E_{n}(\mathbf{k})-\frac{\hbar^{2} k^{2}}{2 m_{0}}\right] u_{n, \mathbf{k}}(\mathbf{r}),
$$

with,

$$
\mathrm{H}=\mathcal{H}_{0}+\frac{\hbar}{m_{0}} \mathbf{k} \cdot \mathbf{p}+\underbrace{\frac{\hbar}{4 m_{0}^{2} c^{2}} \nabla V_{L} \times \mathbf{p} \cdot \boldsymbol{\sigma}+\frac{\hbar}{4 m_{0}^{2} c^{2}} \nabla V_{L} \times \mathbf{k} \cdot \boldsymbol{\sigma},}_{\text {spin-orbital splitting interaction }}
$$

where $\sigma$ is the Pauli spin matrix which has spatial vector components,

$$
\sigma_{x}=\left[\begin{array}{cc}
0 & 1 \\
1 & 0
\end{array}\right], \sigma_{y}=\left[\begin{array}{cc}
0 & -i \\
i & 0
\end{array}\right], \sigma_{z}=\left[\begin{array}{cc}
1 & 0 \\
0 & -1
\end{array}\right]
$$

With this representation, spins can be expressed as the spin vectors,

$$
\uparrow=\left[\begin{array}{l}
1 \\
0
\end{array}\right], \downarrow=\left[\begin{array}{l}
0 \\
1
\end{array}\right] .
$$

Since the crystal momentum $\hbar \mathbf{k}$ is very small compared with the atomic momentum $\mathbf{p}$ in the far interior of the atom where most of the spin-obit interactions occur, k-dependent spin-orbit interaction part is negligible in Kane model.

The next task is to solve exactly the Schrödinger equation Eq. (2.56) using only the eight basis functions, assuming for the moment that the influence of other bands is negligible (four bands with spin). Solving the Schrödinger equation can be done using the basis functions $|S \uparrow\rangle,|S \downarrow\rangle,|X \uparrow\rangle,|X \downarrow\rangle,|Y \uparrow\rangle,|Y \downarrow\rangle,|Z \uparrow\rangle$, $|Z \downarrow\rangle$, however, it is more convenient to choose a linear combination of these functions as the basis from the point of mathematics. 


\section{B. Luttinger-Khon model}

Luttinger-Kohn model $[142,143]$ is used extensively to model VB structures and concerns with the VB structures(class A) only. Unlike Kane model, it does not include the CB wavefunctions in its basis set, and thus does not predict relations between the effective masses and the band gap energies. Although it is much less intuitive compared with Kane model, it does include the effects of the $\mathrm{CB}$ and various other bands (class B) on the mixing of the chosen $\mathrm{VB}$ wavefunctions and on the energies of the bands, through the renormalization introduced by the Löwdin perturbation theory. That is to say, some of the transparency is lost due to the ignorance of $\mathrm{CB}$ and the inclusion of other renormalization effects. The eigenvectors in the Luttinger-Kohn model are the same as those in the Kane model without CB.

With the basis function corresponding to spin up and spin down,

$$
\begin{aligned}
& u_{10}=-\frac{1}{\sqrt{2}}|(X+i Y) \uparrow\rangle=\left|\frac{3}{2}, \frac{3}{2}\right\rangle \\
& u_{20}=-\frac{1}{\sqrt{6}}|(X+i Y) \downarrow\rangle+\sqrt{\frac{2}{3}}|Z \uparrow\rangle=\left|\frac{3}{2}, \frac{1}{2}\right\rangle \\
& u_{30}=\frac{1}{\sqrt{6}}|(X-i Y) \uparrow\rangle+\sqrt{\frac{2}{3}}|Z \downarrow\rangle=\left|\frac{3}{2},-\frac{1}{2}\right\rangle \\
& u_{40}=\frac{1}{\sqrt{2}}|(X-i Y) \downarrow\rangle=\left|\frac{3}{2},-\frac{3}{2}\right\rangle \\
& u_{50}=\frac{1}{\sqrt{3}}|(X+i Y) \downarrow\rangle+\sqrt{\frac{1}{3}}|Z \uparrow\rangle=\left|\frac{1}{2}, \frac{1}{2}\right\rangle \\
& u_{60}=\frac{1}{\sqrt{3}}|(X-i Y) \uparrow\rangle-\sqrt{\frac{1}{3}}|Z \downarrow\rangle=\left|\frac{3}{2},-\frac{1}{2}\right\rangle
\end{aligned}
$$

the Luttinger-Khon Hamiltonian $\mathrm{H}_{\mathrm{LK}}$ is written as, 


$$
H_{L K}=\left(\begin{array}{cccccc|c}
a_{+} & b & c & 0 & i \frac{b}{\sqrt{2}} & -i \sqrt{2} c & \left|\frac{3}{2}, \frac{3}{2}\right\rangle \\
b^{*} & a_{-} & 0 & c & i f & i \sqrt{\frac{3}{2} b} & \left|\frac{3}{2}, \frac{1}{2}\right\rangle \\
c^{*} & 0 & a_{-} & -b & -i \sqrt{\frac{3}{2} b^{*}} & \text { if } & \left|\frac{3}{2},-\frac{1}{2}\right\rangle \\
0 & c^{*} & -b^{*} & a_{+} & -i \sqrt{2} c & -i \frac{b^{*}}{\sqrt{2}} & \left|\frac{3}{2},-\frac{3}{2}\right\rangle \\
-i \frac{b^{*}}{\sqrt{2}} & -i f & \sqrt{\frac{3}{2}} b & i \sqrt{2} c & d & 0 \\
i \sqrt{2} c^{*} & -i \sqrt{\frac{3}{2} b^{*}} & -i f & i \frac{b}{\sqrt{2}} & 0 & d
\end{array}\right),\left|\frac{1}{2}, \frac{1}{2}\right\rangle
$$

where,

$$
\begin{aligned}
& a_{ \pm}=E_{v}-\left(\gamma_{1} \mp 2 \gamma_{2}\right) \frac{\hbar^{2} k_{z}^{2}}{2 m_{0}}-\left(\gamma_{1} \pm \gamma_{2}\right) \frac{\hbar^{2}\left(k_{x}^{2}+k_{y}^{2}\right)}{2 m_{0}}, \\
& b=i 2 \sqrt{3} \gamma_{3} \frac{\hbar^{2}}{2 m_{0}}\left(k_{x}-i k_{y}\right) k_{z}, \\
& c=-\sqrt{3} \frac{\hbar^{2}}{2 m_{0}}\left[\gamma_{2}\left(k_{x}^{2}-k_{y}^{2}\right)-2 i \gamma_{3} k_{x} k_{y}\right] \\
& d=E_{v}-\Delta-\gamma_{1} \frac{\hbar^{2}\left(k_{x}^{2}+k_{y}^{2}+k_{z}^{2}\right)}{2 m_{0}} .
\end{aligned}
$$

Luttinger-Kohn Hamiltonian $\left(H_{L K}\right)$ introduces the Luttinger parameters, $\gamma_{1}, \gamma_{2}$, and $\gamma_{3}$, which can be derived from matrix elements between various bands, but in practice, are experimentally measured parameters. Solving for the eigenvalues and the eigenfunctions of this Luttinger-Kohn Hamiltonian gives a good description of the $\mathrm{HH}, \mathrm{LH}$, and SO bands in diamond and zinc blende semiconductors. 


\section{Principle of FTM for QDs}

The formulation of Hamiltonian matrix in the Fourier domain for QDs is derived here. For the heterostructures, differential operations are usually used to treat with $\mathbf{k}$ in the position-dependent bulk Hamiltonians, then the Eq. (2.61) can be rewritten as,

$$
\mathbf{H}\left(\mathbf{r} ; \hat{k}_{x}, \hat{k}_{y}, \hat{k}_{z}\right) \mathbf{F}(\mathbf{r})=E(\mathbf{k}) \mathbf{F}(\mathbf{r})
$$

Therefore, the Hamiltonian matrix $\mathbf{H}\left(\mathbf{r} ; \hat{k}_{x}, \hat{k}_{y}, \hat{k}_{z}\right)$ can be expanded with Fourier series,

$$
\begin{aligned}
\mathbf{H} & =\sum_{\alpha, \beta}^{x, y, z} H^{(\alpha \beta)}(x, y, z) \hat{k}_{\alpha} \hat{k}_{\beta}+\sum_{\alpha}^{x, y, z} H^{(\alpha)}(x, y, z) \hat{k}_{\alpha}+H^{(0)}(x, y, z) \\
& =\sum_{\alpha, \beta}^{x, y, z} \sum_{q_{x} q_{y} q_{z}} \tilde{H}_{q_{x} q_{y} q_{z}}^{(\alpha \beta)} e^{i\left(q_{x} \kappa_{x} x+q_{y} \kappa_{y} y+q_{z} \kappa_{z} z\right)} \hat{k}_{\alpha} \hat{k}_{\beta} \\
& +\sum_{\alpha}^{x, y, z} \sum_{q_{x} q_{y} q_{z}} \tilde{H}_{q_{x} q_{y} q_{z}}^{(\alpha)} e^{i\left(q_{x} \kappa_{x} x+q_{y} \kappa_{y} y+q_{z} \kappa_{z} z\right.} \hat{k}_{\alpha} \\
& +\sum_{q_{x} q_{y} q_{z}} \tilde{H}_{q_{x} q_{y} q_{z}}^{(0)} e^{i\left(q_{x} \kappa_{x} x+q_{y} \kappa_{y} y+q_{z} \kappa_{z} z\right)}
\end{aligned}
$$

where $q_{x}, q_{y}$ and $q_{z}$ represent the Fourier frequency in $x, y$, and $z$ direction, respectively. Denoting $\left\langle f_{2} \mid f_{1}\right\rangle=(1 / L) \int_{L} f_{2}^{*} f_{1} d z$, it can be derived from Eq. (2.61) that,

$$
\sum_{j}^{V} \sum_{n_{x} n_{y} n_{z}} \mathbf{c}_{j, n_{x} n_{y} n_{z}} \sum_{q_{x} q_{y} q_{z}}\left\langle A A\left|\mathbf{H}\left(\mathbf{r} ; \hat{k}_{x}, \hat{k}_{y}, \hat{k}_{z}\right)\right| B B\right\rangle=\left\langle A A \mid E \sum_{j}^{V} \sum_{n_{x} n_{y} n_{z}} \mathbf{c}_{j, n_{x} n_{y} n_{z}} \cdot B B\right\rangle,
$$

where,

$$
\begin{aligned}
& A A=e^{i k_{x} x+i k_{y} y+i k_{z} z} e^{i\left(m_{x} \kappa_{x} x+m_{y} \kappa_{y} y+m_{z} \kappa_{z} z\right)}, \\
& B B=e^{i k_{x} x+i k_{y} y+i k_{z} z} e^{i\left(n_{x} \kappa_{x} x+n_{y} \kappa_{y} y+n_{z} \kappa_{z} z\right)},
\end{aligned}
$$

With symmetric operator ordering $[52,158]$,

$$
Q \hat{k}_{i} f \rightarrow\left[Q \hat{k}_{i} f+\hat{k}_{i}(Q f)\right] / 2
$$




$$
Q \hat{k}_{i} \hat{k}_{j} f \rightarrow \hat{k}_{j}\left(Q \hat{k}_{i} f\right)+\hat{k}_{i}\left(Q \hat{k}_{j} f\right) / 2
$$

where $\hat{k}_{i}=-i \partial_{i}$ and $\hat{k}_{j}=-i \partial_{j}$. And thus,

$$
\begin{aligned}
& \sum_{q_{x} q_{y} q_{z}}\left\langle A A\left|H^{(x x)}(x, y, z) \hat{k}_{x} \hat{k}_{x}\right| B B\right\rangle= \tilde{H}_{j j^{\prime}, q_{i}=m_{i}-n_{i}}^{(x x)}\left(k_{x}+n_{x} \kappa_{x}\right)\left(k_{x}+m_{x} \kappa_{x}\right), \\
& \sum_{q_{x} q_{y} q_{z}}\left\langle A A\left|H^{(x y)}(x, y, z) \hat{k}_{x} \hat{k}_{y}\right| B B\right\rangle= \frac{1}{2} \tilde{H}_{j j^{\prime}, q_{i}=m_{i}-n_{i}}^{(x y)}\left(k_{y}+n_{y} \kappa_{y}\right)\left(k_{x}+m_{x} \kappa_{x}\right) \\
&+\frac{1}{2} \tilde{H}_{j j^{\prime}, q_{i}=m_{i}-n_{i}}^{(x y)}\left(k_{x}+n_{x} \kappa_{x}\right)\left(k_{y}+m_{y} \kappa_{y}\right) \\
& \sum_{q_{x} q_{y} q_{z}}\left\langle A A\left|H^{(x)}(x, y, z) \hat{k}_{x}\right| B B\right\rangle=\tilde{H}_{j j^{\prime}, q_{i}=m_{i}-n_{i}}^{(x)}\left[k_{x}+\left(m_{x}+n_{x}\right) \kappa_{x} / 2\right], \\
& \sum_{q_{x} q_{y} q_{z}}\left\langle A A\left|H^{(0)}(x, y, z)\right| B B\right\rangle=\tilde{H}_{j j^{\prime}, q_{i}=m_{i}-n_{i},}^{(0)}
\end{aligned}
$$

where $i=x, y, z$. Therefore, the expectation equation, $\mathbf{M}_{j m, j^{\prime} n} \mathbf{c}_{j^{\prime} n}=E \mathbf{c}_{j n}$ is formed, where the matrix element is,

$$
\begin{aligned}
\mathbf{M}_{s t}= & \frac{1}{2} \sum_{\alpha, \beta}^{x, y, z} \tilde{H}_{j j^{\prime}, q_{i}=m_{i}-n_{i}}^{(\alpha)}\left[\left(k_{\beta}+n_{\beta} \kappa_{\beta}\right)\left(k_{\alpha}+n_{\alpha} \kappa_{\alpha}\right)+\left(k_{\alpha}+n_{\alpha} \kappa_{\alpha}\right)\left(k_{\beta}+n_{\beta} \kappa_{\beta}\right)\right] \\
& +\frac{1}{2} \sum_{\alpha}^{x, y, z} \tilde{H}_{j j^{\prime}, q_{i}=m_{i}-n_{i}}^{(\alpha)}\left[2 k_{\alpha}+\left(m_{\alpha}+n_{\alpha}\right) \kappa_{\alpha}\right] \\
& +\tilde{H}_{j j j^{\prime}, q_{i}=m_{i}-n_{i}}^{(0)}
\end{aligned}
$$




\section{Singular value of analytical Fourier-transform expressions for several QD shapes}

All the analytical Fourier-transform expressions used in this thesis are referred after Ref. [67] and Ref. [116], however, the singular values of the analytical Fourier-transform expressions for several QD shapes (i.e. sphere, cylinder, cube, pyramid, and truncated pyramid) have not been given. Therefore, this part is supplemented here. For those other shapes not listed here, numerical computation is recommended.

\section{1 Sphere}

$$
\tilde{\Lambda}(\xi)=\frac{4 \pi}{\xi}\left[\frac{\sin (\xi \cdot R)}{\xi^{2}}-\frac{\cos (\xi \cdot R)}{\xi}\right],
$$

where $R$ is the sphere radius, the origin of the coordinates is at the center of the sphere, and the singular value for $\xi=0$ is $\tilde{\Lambda}(0)=4 \pi R^{3} / 3$.

\section{2 Cylinder}

$$
\tilde{\Lambda}\left(\xi_{n}\right)=\tilde{\Lambda}_{/ /}\left(\xi_{/ /}, D\right) \cdot \tilde{\Lambda}_{3}\left(\xi_{3}, h\right)
$$

where,

$$
\begin{aligned}
& \tilde{\Lambda}_{/ /}\left(\xi_{/ /}, D\right)=\frac{\pi D}{\xi_{/ /}} J_{1}\left(\xi_{/ /} D / 2\right) \\
& \tilde{\Lambda}_{3}\left(\xi_{3}, h\right)=\frac{\sin \left(\xi_{3} h / 2\right)}{\xi_{3} / 2}
\end{aligned}
$$

The origin of the coordinates is at the center of QD. The singular values for $\xi_{n}=0$ are $\tilde{\Lambda}_{/ /}(0, D)=\pi D^{2} / 4$ and $\tilde{\Lambda}_{3}(0, h)=h$, respectively

\section{3 Cube}

$$
\tilde{\Lambda}\left(\xi_{n}\right)=\tilde{\Lambda}_{1}\left(\xi_{1}, a_{x}\right) \cdot \tilde{\Lambda}_{2}\left(\xi_{2}, a_{y}\right) \cdot \tilde{\Lambda}_{3}\left(\xi_{3}, a_{z}\right)
$$

where, 


$$
\begin{aligned}
& \tilde{\Lambda}_{1}\left(\xi_{1}, a_{x}\right)=\frac{\sin \left(\xi_{1} a_{x} / 2\right)}{\xi_{1} / 2} \\
& \tilde{\Lambda}_{2}\left(\xi_{2}, a_{y}\right)=\frac{\sin \left(\xi_{2} a_{y} / 2\right)}{\xi_{2} / 2} . \\
& \tilde{\Lambda}_{3}\left(\xi_{3}, a_{z}\right)=\frac{\sin \left(\xi_{3} a_{z} / 2\right)}{\xi_{3} / 2}
\end{aligned}
$$

The origin of the coordinates is at the center of $\mathrm{QD}$, and $a_{x}, a_{y}$ and $a_{z}$ are dimensions along $x, y$, and $z$ directions, respectively. The singular values for $\xi_{n}=0$ are $\tilde{\Lambda}_{1}\left(0, a_{x}\right)=a_{x}, \tilde{\Lambda}_{2}\left(0, a_{y}\right)=a_{y}$, and $\tilde{\Lambda}_{3}\left(0, a_{z}\right)=a_{z}$, respectively.

\section{4 Pyramid}

$$
\begin{aligned}
\tilde{\Lambda}_{p y r}\left(\xi_{n}, L_{x}, L_{y}\right)= & \tilde{\Lambda}_{1}\left(\xi_{1}, \xi_{2}, \xi_{3}, L_{x}, L_{y}\right)+\tilde{\Lambda}_{1}\left(-\xi_{1},-\xi_{2}, \xi_{3}, L_{x}, L_{y}\right) \\
& +\tilde{\Lambda}_{1}\left(\xi_{2},-\xi_{1}, \xi_{3}, L_{y}, L_{x}\right)+\tilde{\Lambda}_{1}\left(-\xi_{2}, \xi_{1}, \xi_{3}, L_{y}, L_{x}\right)
\end{aligned},
$$

with

$$
\tilde{\Lambda}_{1}\left(\xi_{1}, \xi_{2}, \xi_{3}, L_{x}, L_{y}\right)=\frac{1}{-\xi_{2} \xi_{3}}\left\{\begin{array}{c}
e^{-i \xi_{3} h}\left[\begin{array}{c}
I_{e, 0}\left(\frac{L_{x}}{2},-\xi_{1}-\frac{L_{y}}{L_{x}} \xi_{2}+\frac{2 h}{L_{x}} \xi_{3}\right) \\
-I_{e, 0}\left(\frac{L_{x}}{2},-\xi_{1}+\frac{L_{y}}{L_{x}} \xi_{2}+\frac{2 h}{L_{x}} \xi_{3}\right)
\end{array}\right] \\
-I_{e, 0}\left(\frac{L_{x}}{2},-\xi_{1}-\frac{L_{y}}{L_{x}} \xi_{2}\right)+I_{e 0}\left(\frac{L_{x}}{2},-\xi_{1}+\frac{L_{y}}{L_{x}} \xi_{2}\right)
\end{array}\right\}
$$

where $I_{e, m}(a, \xi)=\int_{0}^{a} x^{m} e^{i \xi x} d x=\frac{a^{m}}{i \zeta} e^{i \zeta a}-\frac{m}{i \zeta} I_{e, m-1}(a, \zeta)$ and $I_{e, 0}(a, \xi)=\frac{1}{i \zeta}\left(e^{i \zeta a}-1\right)$,

$L_{x}$ and $L_{y}$ are the pyramid base dimensions (the base is assumed to form a rectangular), $h$ is pyramid height. The origin of the coordinate system is at the base center of the pyramid. Singular values of $I_{e, m}(a, \xi)$ as $m=0,1,2$ are as following,

$$
\begin{aligned}
& I_{e, 0}(a, 0)=a \\
& I_{e, 1}(a, 0)=\frac{a^{2}}{2} . \\
& I_{e, 2}(a, 0)=\frac{a^{3}}{3}
\end{aligned}
$$


Therefore singular values for $\tilde{\Lambda}_{1}\left(\xi_{1}, \xi_{2}, \xi_{3}, L_{x}, L_{y}\right)$ are as following,

$$
\begin{aligned}
\tilde{\Lambda}_{1}\left(\xi_{1}, \xi_{2}, 0, L_{x}, L_{y}\right)= & \frac{h}{-i \xi_{2}}\left[I_{e 0}\left(\frac{L_{x}}{2},-\xi_{1}-\frac{L_{y}}{L_{x}} \xi_{2}\right)-I_{e 0}\left(\frac{L_{x}}{2},-\xi_{1}+\frac{L_{y}}{L_{x}} \xi_{2}\right)\right] \\
& +\frac{2 h}{i L_{x} \xi_{2}}\left[I_{e, 1}\left(\frac{L_{x}}{2},-\xi_{1}-\frac{L_{y}}{L_{x}} \xi_{2}\right)-I_{e, 1}\left(\frac{L_{x}}{2},-\xi_{1}+\frac{L_{y}}{L_{x}} \xi_{2}\right)\right] \\
\tilde{\Lambda}_{1}\left(\xi_{1}, 0, \xi_{3}, L_{x}, L_{y}\right)= & \frac{2}{-i \xi_{3}} \cdot \frac{L_{y}}{L_{x}}\left[e^{-i \xi_{z} h} \cdot I_{e, 1}\left(\frac{L_{x}}{2},-\xi_{1}+\frac{2 h}{L_{x}} \xi_{3}\right)-I_{e, 1}\left(\frac{L_{x}}{2},-\xi_{1}\right)\right], \\
\tilde{\Lambda}_{1}\left(\xi_{1}, 0,0, L_{x}, L_{y}\right)= & 2 h\left[\frac{L_{y}}{L_{x}} \cdot I_{e, 1}\left(\frac{L_{x}}{2},-\xi_{1}\right)-\frac{2 L_{y}}{L_{x}^{2}} \cdot I_{e, 2}\left(\frac{L_{x}}{2},-\xi_{1}\right)\right]
\end{aligned}
$$

\section{5 Truncated pyramid}

$$
\tilde{\Lambda}\left(\xi_{n}\right)=\tilde{\Lambda}_{p y r}\left(\xi_{n}, L_{x}^{b}, L_{y}^{b}, H\right)-e^{-i \xi_{3} h} \tilde{\Lambda}_{p y r}\left(\xi_{n}, L_{x}^{t}, L_{y}^{b t}, H-h\right),
$$

where $L_{x}^{b}$ and $L_{y}^{b}$ are the base lengths, $L_{x}^{t}$ and $L_{y}^{t}$ are the dimensions of the top face, $h$ is the height of truncated pyramid, $\widetilde{\Lambda}_{p y r}$ is the Fourier transform of a pyramid (see Eq. (D.6)-(D.11)), $H=L_{x}^{b} h /\left(L_{x}^{b}-L_{x}^{t}\right)$. The origin of the coordinate system is at the base center of the truncated pyramid. 


\section{E. Derivation of FTM with BF operator ordering}

With the QW as the illustration example, the formulation of Hamiltonian matrix with $\mathrm{BF}$ operator ordering in the Fourier domain is derived here. For the QWs, differential operations are usually used to treat with $k_{z}$ in the positiondependent bulk Hamiltonians, then the Eq. (5.10) can be rewritten as,

$$
\mathbf{H}\left(\mathbf{r} ; \mathbf{k}_{\|,}, \hat{k}_{z}\right) \mathbf{F}(\mathbf{r})=E(\mathbf{k}) \mathbf{F}(\mathbf{r}) .
$$

Therefore, the Hamiltonian matrix $\mathbf{H}\left(\mathbf{r} ; \mathbf{k}_{\|}, \hat{k}_{z}\right)$ can be expanded with Fourier series,

$$
\begin{aligned}
\mathbf{H} & =H^{(z z)}(z) \hat{k}_{z}^{2}+H_{L}^{(z)}(z) \hat{k}_{z}+\hat{k}_{z} H_{R}^{(z)}(z)+H^{(0)}(z) \\
& =\sum_{q} \tilde{H}_{q}^{(z z)} e^{i q \cdot k_{z} \cdot z} \hat{k}_{z}^{2} \\
& +\sum_{q} \tilde{H}_{q, L}^{(z)} e^{i q \cdot k_{z} \cdot z} \hat{k}_{z}++\sum_{q} \hat{k}_{z} \tilde{H}_{q, R}^{(z)} e^{i q \cdot k_{z} \cdot z} \\
& +\sum_{q} \tilde{H}_{q}^{(0)} e^{i q \cdot k_{z} \cdot z}
\end{aligned}
$$

where $q$ represents the Fourier frequency the quantized $z$ direction.

Denoting $\left\langle f_{2} \mid f_{1}\right\rangle=(1 / L) \int_{L} f_{2}^{*} f_{1} d z$, it can be derived from Eq. (E.1) that,

$$
\sum_{v=1}^{V} \sum_{n_{z}} c_{v, n_{z}} \sum_{q}\langle A A|\mathbf{H}| B B\rangle=\left\langle A A \mid E \sum_{v=1}^{V} \sum_{n_{z}} c_{v, n_{z}} \cdot B B\right\rangle,
$$

where,

$$
\begin{aligned}
& A A=e^{i k_{x} x+i k_{y} y} e^{i m_{z} K_{z} z}, \\
& B B=e^{i k_{x} x+i k_{y} y} e^{i n_{z} K_{z} z},
\end{aligned}
$$

and $u, v=1,2, \ldots, V$. With BF operator ordering and $\hat{k}_{i} \rightarrow-i \partial_{i}$ and $\hat{k}_{j} \rightarrow-i \partial_{j}$, we have,

$$
\begin{aligned}
& \sum_{q}\left\langle A A\left|\hat{k}_{z} H^{(z z)}(z) \hat{k}_{z}\right| B B\right\rangle=\tilde{H}_{u v, q=m-n}^{(z z)}\left(k_{z}+m_{z} \kappa_{z}\right)\left(k_{z}+n_{z} \kappa_{z}\right), \\
& \sum_{q}\left\langle A A\left|H_{L}^{(z)}(z) \hat{k}_{z}\right| B B\right\rangle=\tilde{H}_{u v, q=m-n, L}^{(z)}\left(k_{z}+n_{z} \kappa_{z}\right), \\
& \sum_{q}\left\langle A A\left|\hat{k}_{z} H_{R}^{(z)}(z)\right| B B\right\rangle=\tilde{H}_{u v, q=m-n, R}^{(z)}\left(k_{z}+m_{z} \kappa_{z}\right),
\end{aligned}
$$




$$
\sum_{q}\left\langle A A\left|H^{(0)}(z)\right| B B\right\rangle=\tilde{H}_{u v, q=m-n}^{(0)}
$$

Therefore, we form the expectation equation, $\mathbf{M}_{u m, v n} \mathbf{c}_{v n}=E \mathbf{c}_{v n}$, where the matrix element for QW is,

$$
\begin{aligned}
\mathbf{M}_{u m, v n} & =\tilde{H}_{u v, q=m-n}^{(z z)}\left(k_{z}+m_{z} \kappa_{z}\right)\left(k_{z}+n_{z} \kappa_{z}\right) \\
& +\tilde{H}_{u v, q=m-n, L}^{(z)}\left(k_{z}+n_{z} \kappa_{z}\right) \\
& +\tilde{H}_{u v, q=m-n, R}^{(z)}\left(k_{z}+m_{z} \kappa_{z}\right) \\
& +\tilde{H}_{u v, q=m-n}^{(0)}
\end{aligned}
$$

In the similar way, the momentum matrix for heterostructures can be expressed as,

$$
\begin{aligned}
\mathbf{M}_{u m, v n} & =\sum_{\alpha, \beta} \tilde{H}_{u v, q_{i}=m_{i}-n_{i}}^{(\alpha \beta)}\left(k_{\alpha}+m_{\alpha} \kappa_{\alpha}\right)\left(k_{\beta}+n_{\beta} \kappa_{\beta}\right) \\
& +\sum_{\alpha} \tilde{H}_{u v, q_{i}=m_{i}-n_{i}, L}^{(\alpha)}\left(k_{\alpha}+n_{\alpha} \kappa_{\alpha}\right) \\
& +\sum_{\alpha} \tilde{H}_{u v, q_{i}=m_{i}-n_{i}, R}^{(\alpha)}\left(k_{\alpha}+m_{\alpha} \kappa_{\alpha}\right) \\
& +\tilde{H}_{u v, q_{i}=m_{i}-n_{i}}^{(0)}
\end{aligned}
$$




\section{F. Hamiltonians}

\section{F. 1 Eight-band Hamiltonian with SYM operator ordering}

Table F-1 Basis function of the eight-band Hamiltonian matrix with the SYM operator ordering [107, 153, 154]

$\begin{array}{ll}C B: u_{1}=|S \downarrow\rangle & u_{2}=|S \uparrow\rangle \\ L H: u_{3}=\left|\frac{i}{\sqrt{6}}[2 Z \uparrow-(X+i Y) \downarrow]\right\rangle \equiv\left|\frac{1}{2}\right\rangle & u_{6}=\left|\frac{i}{\sqrt{6}}[2 Z \downarrow+(X-i Y) \uparrow]\right\rangle \equiv\left|-\frac{1}{2}\right\rangle \\ H H: u_{4}=\left|\frac{i}{\sqrt{2}}(X+i Y) \uparrow\right\rangle \equiv\left|\frac{3}{2}\right\rangle & u_{5}=\left|-\frac{i}{\sqrt{2}}(X-i Y) \downarrow\right\rangle \equiv\left|-\frac{3}{2}\right\rangle \\ S O: u_{7}=\left|\frac{i}{\sqrt{3}}[Z \downarrow-(X-i Y) \uparrow]\right\rangle \equiv\left|-\frac{7}{2}\right\rangle & u_{8}=\left|-\frac{i}{\sqrt{3}}[Z \uparrow+(X+i Y) \downarrow]\right\rangle \equiv\left|\frac{7}{2}\right\rangle\end{array}$

Table F-2 Kinetic eight-band Hamiltonian matrix with the SYM operator ordering [107, 153, 154].

$\mathbf{H}_{8 \times 8, k}^{S Y M}=\left[\begin{array}{cccccccc}A & 0 & V^{*} & 0 & \sqrt{3} V & -\sqrt{2} U & -U & \sqrt{2} V^{*} \\ 0 & A & -\sqrt{2} U & -\sqrt{3} V^{*} & 0 & -V & \sqrt{2} V & U \\ V & -\sqrt{2} U & -P+Q & -S^{*} & R & 0 & \sqrt{3 / 2} S & -\sqrt{2} Q \\ 0 & -\sqrt{3} V & -S & -P-Q & 0 & R & -\sqrt{2} R & \sqrt{1 / 2} S \\ \sqrt{3} V^{*} & 0 & R^{*} & 0 & -P-Q & S^{*} & \sqrt{1 / 2} S^{*} & \sqrt{2} R^{*} \\ -\sqrt{2} U & -V^{*} & 0 & R^{*} & S & -P+Q & \sqrt{2} Q & \sqrt{3 / 2} S^{*} \\ -U & \sqrt{2} V^{*} & \sqrt{3 / 2} S^{*} & -\sqrt{2} R^{*} & \sqrt{1 / 2} S & \sqrt{2} Q & Z & 0 \\ \sqrt{2} V & U & -\sqrt{2} Q & \sqrt{1 / 2} S^{*} & \sqrt{2} R & \sqrt{3 / 2} S & 0 & Z\end{array}\right]$

with,

$$
\begin{aligned}
& A=E_{c}+G_{c}\left(k_{x}^{2}+k_{y}^{2}+k_{z}^{2}\right) \\
& P=-E_{v}+\gamma_{1}\left(k_{x}^{2}+k_{y}^{2}+k_{z}^{2}\right) \\
& Q=\gamma_{2}\left(k_{x}^{2}+k_{y}^{2}-2 k_{z}^{2}\right) \\
& Z=E_{v}-\Delta-\gamma_{1}\left(k_{x}^{2}+k_{y}^{2}+k_{z}^{2}\right) \\
& U=\frac{1}{\sqrt{3}} G_{c v} k_{z} \\
& V=\frac{1}{\sqrt{6}} G_{c v}\left(k_{x}-i k_{y}\right)
\end{aligned}
$$




$$
\begin{aligned}
& R=-\sqrt{3}\left[\gamma_{2}\left(k_{x}^{2}-k_{y}^{2}\right)-2 i \gamma_{3} k_{x} k_{y}\right] \\
& S=2 \sqrt{3} \gamma_{3} k_{z}\left(k_{x}-i k_{y}\right) \\
& G_{c}=\left[\frac{m_{0}}{m_{c}}-\frac{E_{p}}{3}\left(\frac{2}{E_{g}}+\frac{1}{E_{g}+\Delta}\right)\right] \\
& G_{c v}=\sqrt{E_{p}}
\end{aligned}
$$

Table F-3 Strain Hamiltonian matrix of the eight-band Hamiltonian matrix [107, $153,154]$.

$\mathbf{H}_{8 \times 8, s}^{S Y M}=\left[\begin{array}{cccccccc}a & 0 & -v^{*} & 0 & -\sqrt{3} v & \sqrt{2} u & u & -\sqrt{2} v^{*} \\ 0 & a & \sqrt{2} u & \sqrt{3} v^{*} & 0 & v & -\sqrt{2} v & -u \\ -v & \sqrt{2} u & -p+q & -s^{*} & r & 0 & \sqrt{3 / 2} s & -\sqrt{2} q \\ 0 & \sqrt{3} v & -s & -p-q & 0 & r & -\sqrt{2} r & \sqrt{1 / 2} s \\ -\sqrt{3} v^{*} & 0 & r^{*} & 0 & -p-q & s^{*} & \sqrt{1 / 2} s^{*} & \sqrt{2} r^{*} \\ \sqrt{2} u & v^{*} & 0 & r^{*} & s & -p+q & \sqrt{2} q & \sqrt{3 / 2} s^{*} \\ u & -\sqrt{2} v^{*} & \sqrt{3 / 2} s^{*} & -\sqrt{2} r^{*} & \sqrt{1 / 2} s & \sqrt{2} q & -a_{v} e & 0 \\ -\sqrt{2} v & -u & -\sqrt{2} q & \sqrt{1 / 2} s^{*} & \sqrt{2} r & \sqrt{3 / 2} s & 0 & -a_{v} e\end{array}\right]$

with,

$$
\begin{aligned}
a & =a_{c} e_{h} \\
p & =a_{v} e_{h} \\
q & =b e_{b} \\
u & =\sqrt{\frac{1}{3}} G_{c v} \sum_{j} e_{3 j} \hat{k}_{j} \\
r & =\frac{\sqrt{3}}{2} b\left(e_{11}-e_{22}\right)-i d e_{12} \\
v & =\sqrt{\frac{E_{P}}{6}} \sum_{j}\left(e_{1 j}-i e_{2 j}\right) \hat{k}_{j} \\
s & =-d\left(e_{13}-i e_{23}\right)
\end{aligned}
$$

\section{F. 2 Six-band Hamiltonian with SYM operator ordering}


Table F-4 Basis function of the six-band Hamiltonian with the SYM operator ordering [106].

$$
\begin{aligned}
& H H:|1\rangle=\alpha^{*}\left|-\frac{X+i Y}{\sqrt{2}} \uparrow\right\rangle+\alpha\left|\frac{X-i Y}{\sqrt{2}} \downarrow\right\rangle|4\rangle=\alpha^{*}\left|-\frac{X+i Y}{\sqrt{2}} \uparrow\right\rangle-\alpha\left|\frac{X-i Y}{\sqrt{2}} \downarrow\right\rangle \\
& L H:|2\rangle=\beta\left|\frac{X-i Y}{\sqrt{2}} \uparrow\right\rangle+\beta^{*}\left|-\frac{X+i Y}{\sqrt{2}} \downarrow\right\rangle|5\rangle=\beta\left|\frac{X-i Y}{\sqrt{2}} \uparrow\right\rangle-\beta^{*}\left|-\frac{X+i Y}{\sqrt{2}} \downarrow\right\rangle \\
& C H:|3\rangle=\beta^{*}|Z \uparrow\rangle+\beta|Z \downarrow\rangle, \quad|6\rangle=-\beta^{*}|Z \uparrow\rangle+\beta|Z \downarrow\rangle
\end{aligned}
$$

with,

$$
\begin{aligned}
& \alpha=\frac{1}{\sqrt{2}} \exp \left[i\left(\frac{3 \pi}{4}+\frac{3 \phi}{2}\right)\right] \quad \beta=\frac{1}{\sqrt{2}} \exp \left[i\left(\frac{\pi}{4}+\frac{\phi}{2}\right)\right] \\
& \phi=\tan ^{-1}\left(k_{y} / k_{x}\right)
\end{aligned}
$$

Table F-5 The six-band Hamiltonian with the SYM operator ordering. It is composed by two $3 \times 3$ Hamiltonian matrices [106].

$\mathbf{H}_{6 \times 6}^{S Y M}=\left[\begin{array}{cccccc}F & -K_{t} & -i H_{t} & 0 & 0 & 0 \\ K_{t} & G & \Delta-i H_{t} & 0 & 0 & 0 \\ i H_{t} & \Delta+i H_{t} & \lambda & 0 & 0 & 0 \\ 0 & 0 & 0 & F & K_{t} & i H_{t} \\ 0 & 0 & 0 & K_{t} & G & \Delta+i H_{t} \\ 0 & 0 & 0 & -i H_{t} & \Delta-i H_{t} & \lambda\end{array}\right]=\left[\begin{array}{cc}\mathbf{H}^{U} & 0 \\ 0 & \mathbf{H}^{L}\end{array}\right]$

with,

$$
\begin{aligned}
& F=\Delta_{1}+\Delta_{2}+\lambda+\theta \\
& G=\Delta_{1}-\Delta_{2}+\lambda+\theta \\
& \lambda=\frac{\hbar^{2}}{2 m_{0}}\left[A_{1} k_{z}^{2}+A_{2}\left(k_{x}^{2}+k_{y}^{2}\right)\right]+\lambda_{\varepsilon} \\
& \theta=\frac{\hbar^{2}}{2 m_{0}}\left[A_{3} k_{z}^{2}+A_{4}\left(k_{x}^{2}+k_{y}^{2}\right)\right]+\theta_{\varepsilon} \\
& \lambda_{\varepsilon}=D_{1} e_{33}+D_{2}\left(e_{11}+e_{22}\right) \\
& \theta_{\varepsilon}=D_{3} e_{33}+D_{4}\left(e_{11}+e_{22}\right) \\
& K_{t}=\frac{\hbar^{2}}{2 m_{0}} A_{5} k_{t}^{2} \\
& H_{t}=\frac{\hbar^{2}}{2 m_{0}} A_{6} k_{t} k_{z} \\
& \Delta=\sqrt{2} \Delta_{3}
\end{aligned}
$$




\section{F. 3 Six-band Hamiltonian with the BF operator ordering}

Table F-6 Basis function of the six-band Hamiltonian matrix with the BF operator ordering [160]/
$C B: u_{1}=\left|-\frac{X+i Y}{\sqrt{2}} \uparrow\right\rangle$
$u_{4}=\left|\frac{X-i Y}{\sqrt{2}} \downarrow\right\rangle$
$H H: u_{2}=\left|\frac{X-i Y}{\sqrt{2}} \uparrow\right|$
$u_{5}=\left|-\frac{X+i Y}{\sqrt{2}} \downarrow\right\rangle$
$C H: u_{3}=|Z \uparrow\rangle$
$u_{6}=|Z \downarrow\rangle$

Table F-7 Six-band Hamiltonian matrix with the BF operator ordering [74].

$H_{6 \times 6}^{B F}=\left[\begin{array}{llllll}F+\delta & \kappa^{*} & \varsigma & & & \\ \kappa & G-\delta & -\varsigma^{*} & & & \Delta \\ \eta^{*} & -\eta & \lambda & & \Delta & \\ & & & F-\delta & \kappa & -\varsigma^{*} \\ & & \Delta & \kappa^{*} & G+\delta & \varsigma \\ & \Delta & & -\eta & \eta^{*} & \lambda\end{array}\right]$

with,

$$
\begin{aligned}
& F=\Delta_{1}+\Delta_{2}+\lambda+\theta \\
& G=\Delta_{1}-\Delta_{2}+\lambda+\theta \\
& \lambda=\hat{k}_{z} A_{1} \hat{k}_{z}+\hat{k}_{x} A_{2} \hat{k}_{x}+\hat{k}_{y} A_{2} \hat{k}_{y} \\
& \theta=\hat{k}_{z} A_{3} \hat{k}_{z}+\hat{k}_{x} A_{4} \hat{k}_{x}+\hat{k}_{y} A_{4} \hat{k}_{y} \\
& \kappa=-\hat{k}_{x} A_{5} \hat{k}_{x}+\hat{k}_{y} A_{5} \hat{k}_{y}+i\left(\hat{k}_{x} A_{5} \hat{k}_{y}+\hat{k}_{y} A_{5} \hat{k}_{x}\right) \\
& \eta=-\hat{k}_{z} A_{6}^{+}\left(\hat{k}_{x}+i \hat{k}_{y}\right)-\left(\hat{k}_{x}+i \hat{k}_{y}\right) A_{6}^{-} \hat{k}_{z} \\
& \varsigma=-\hat{k}_{z} A_{6}^{-}\left(\hat{k}_{x}+i \hat{k}_{y}\right)-\left(\hat{k}_{x}+i \hat{k}_{y}\right) A_{6}^{+} \hat{k}_{z} \\
& \delta=i \hat{k}_{y}\left(A_{5}^{+}-A_{5}^{-}\right) \hat{k}_{x}-i \hat{k}_{x}\left(A_{5}^{+}-A_{5}^{-}\right) \hat{k}_{y} \\
& \Delta=2 \sqrt{3} \Delta_{3} \\
& A_{5}^{-}=\frac{-A_{5}+A_{2}+A_{4}-1}{2} \\
& A_{6}^{-}=\frac{A_{1}+A_{3}-1}{\sqrt{2}} \\
& A_{i}=A_{i}^{+}+A_{i}^{-}
\end{aligned}
$$




\section{AUTHOR'S PUBLICATIONS}

\section{Journal papers:}

1. Q. J. Zhao, and T. Mei, "Analysis of electronic structures of quantum dots using meshless Fourier transform k·p method", J. Appl. Phys., 109, 063101063113(2011).

2. Q. J. Zhao, T. Mei, D. H. Zhang, and O. Kurniawan, "Analysis of wetting layer effect on electronic structures of truncated-pyramid quantum dots", Opt. Quant. Electron., 42, 705 -711(2011).

3. Q. J. Zhao, T. Mei, and D. H. Zhang, "Elimination of spurious solutions in the $\mathrm{k} \cdot \mathrm{p}$ theory with Fourier transform technique and Burt-Foreman operator ordering," J. Appl. Phys., 111,053702-053708 (2012).

\section{Conference papers:}

1. Q. J. Zhao, T. Mei, D. H. Zhang, and O. Kurniawan, “Analysis of redshift in InAs-InGaAs quantum dots with Fourier-transform based k·p method"

OSA-IEEE-COS Topical Meeting on Advances in Optoelectronics and Micro/nano-optics(AOM), 3-6 December, 2010, Guangzhou, PRC.

2. Q. J. Zhao, T. Mei, and D. H. Zhang, "Analysis of wetting layer Effect on electronic structures of truncated-pyramid quantum dots",10th International Conference on numerical Simulation of Optoelectronic Devices (NUSOD), 6-9 September, 2010, Atlanta, USA.

3. Q. J. Zhao, T. Mei, and X. G. Tu, “Application of Fourier transform-based $\mathrm{K} \cdot \mathrm{P}$ method to semiconductor superlattices", International Conference on 
Materials for Advanced Technologies (ICMAT), 28 June - 3 July, 2009, Singapore.

4. T. Mei and Q. J. Zhao, "Fourier transform method for $k \cdot p$ calculation", International Conference on Transport and Optical Properties of Nanomaterials (ICTOPON), 5-8 January, 2009, Allahabad, Indian. 


\section{REFERENCES}

[1] M. A. Herman, W. Richter, and H. Sitter, Epitaxy: physical principles and technical implementation. Verlag: Springer, 2004.

[2] D. Bimberg and U. W. Pohl, "Quantum dots: promises and accomplishments," Mater. Today, vol. 14, pp. 388-397, 2011.

[3] J. J. Coleman, "Strained-layer InGaAs quantum-well heterostructure lasers," IEEE J. Sel. Top. Quant., vol. 6, pp. 1008-1013, 2000.

[4] P. Aivaliotis, S. Menzel, E. A. Zibik, J. W. Cockburn, L. R. Wilson, and M. Hopkinson, "Energy level structure and electron relaxation times in InAs Inx Ga1-x As quantum dot-in-a-well structures," Appl. Phys. Lett., vol. 91, pp. 253502-253504, 2007.

[5] C. Gies, J. Wiersig, M. Lorke, and F. Jahnke, "Semiconductor model for quantum-dot-based microcavity lasers," Phys. Rev. A, vol. 75, pp. 013803$013813,2007$.

[6] J. Faist, "Semiconductor Lasers: Lasing high in k-space," Nat. Photonics, vol. 3, pp. 11-12, 2009.

[7] M. G. Thompson, A. R. Rae, M. Xia, R. V. Penty, and I. H. White, "InGaAs quantum-dot mode-locked laser diodes," IEEE J. Sel. Top. Quant., vol. 15, pp. 661-672, 2009.

[8] R. Kohler, A. Tredicucci, F. Beltram, H. E. Beere, E. H. Linfield, A. G. Davies, D. A. Ritchie, R. C. Iotti, and F. Rossi, "Terahertz semiconductorheterostructure laser," Nature, vol. 417, pp. 156-159, 2002.

[9] F. Qian, S. Gradečak, Y. Li, C. Y. Wen, and C. M. Lieber, "Core/multishell nanowire heterostructures as multicolor, high-efficiency light-emitting diodes," Nano Lett., vol. 5, pp. 2287-2291, 2005.

[10] M. Zhang, P. Bhattacharya, J. Singh, and J. Hinckley, "Direct measurement of auger recombination in In0.1 Ga $0.9 \mathrm{~N} / \mathrm{GaN}$ quantum wells and its impact on the efficiency of In $0.1 \mathrm{Ga} 0.9 \mathrm{~N} / \mathrm{GaN}$ multiple quantum well light emitting diodes," Appl. Phys. Lett., vol. 95, pp. 201108-201110, 2009.

[11] M. Khoshnegar, M. Sodagar, A. Eftekharian, and S. Khorasani, "Design of a GaN white light-emitting diode through envelope function analysis," IEEE J. Quantum Elect., vol. 46, pp. 228-237, 2010.

[12] L. J. Yan, C. C. Yang, M. L. Lee, S. J. Tu, C. S. Chang, and J. K. Sheu, "AlGaInP/GaP heterostructures bonded with si substrate to serve as solar cells and light emitting diodes," J. Electronchem. Sco., vol. 157, pp. H452H454, 2010.

[13] J. H. Lee, "High-Power InGaN-Based LED With Tunneling-JunctionInduced Two-Dimensional Electron Gas at AlGaN/GaN Heterostructure," IEEE T. Electron Dev., In press.

[14] F. A. Ponce and D. P. Bour, "NItride-based semiconductors for blue and green light-emitting devices," Nature, vol. 386, pp. 351-359, 1997.

[15] W. H. Chang, W. Y. Chen, H. S. Chang, T. P. Hsieh, J. I. Chyi, and T. M. Hsu, "Efficient single-photon sources based on low-density quantum dots in photonic-crystal nanocavities," Phys. Rev. Lett., vol. 96, pp. 1-4, 2006. 
[16] M. J. Stevens, R. H. Hadfield, R. E. Schwall, S. W. Nam, and R. P. Mirin, "Quantum dot single photon sources studied with superconducting single photon detectors," IEEE J. Sel. Top. Quant., vol. 12, pp. 1255-1267, 2006.

[17] A. J. Shields, "Semiconductor quantum light sources," Nat. Photonics, vol. 1, pp. 215-223, 2007.

[18] J. Claudon, J. Bleuse, N. S. Malik, M. Bazin, P. Jaffrennou, N. Gregersen, C. Sauvan, P. Lalanne, and J. M. Gérard, "A highly efficient single-photon source based on a quantum dot in a photonic nanowire," Nat. Photonics, vol. 4, pp. 174-177, 2010.

[19] M. T. Rakher, L. Ma, O. Slattery, X. Tang, and K. Srinivasan, "Quantum transduction of telecommunications-band single photons from a quantum dot by frequency upconversion," Nat. Photonics, vol. 4, pp. 786-791, 2010.

[20] J. Heinrich, A. Huggenberger, T. Heindel, S. Reitzenstein, S. Hofling, L. Worschech, and A. Forchel, "Single photon emission from positioned GaAs/AlGaAs photonic nanowires," Appl. Phys. Lett., vol. 96, pp. 211117 $211119,2010$.

[21] R. W. Kelsall, I. W. Hamley, and M. Geoghegan, Nanoscal science and technology. West Sussex: John Wiley and Sons, 2005.

[22] S. L. Chuang, Physics of optoelectronic device. New York: Wiley, 1995.

[23] T. Chakraborty, Quantum dots: A survey of the properties of artificial atoms. Amsterdam: Elsevier Science, 1999.

[24] D. Bimberg, M. Grundmann, and N. N. Ledentsov, Quantum dot heterostructures. Sussex: Wiley, 1999.

[25] J. W. Matthews, Epitaxial Growth. New York: Academic Press.

[26] O. K. Andersen and O. Jepsen, "Explicit, first-principles tight-binding theory," Phys. Rev. Lett., vol. 53, pp. 2571-2574, 1984.

[27] R. Santoprete, B. Koiller, R. B. Capaz, P. Kratzer, Q. K. K. Liu, and M. Scheffler, "Tight-binding study of the influence of the strain on the electronic properties of InAs/GaAs quantum dots," Phys. Rev. B, vol. 68, pp. 235311-235319, 2003.

[28] S. Lee, F. Oyafuso, P. von Allmen, and G. Klimeck, "Boundary conditions for the electronic structure of finite-extent embedded semiconductor nanostructures," Phys. Rev. B, vol. 69, pp. 045316-045323, 2004.

[29] W. Jaskólski, M. Zieliński, G. W. Bryant, and J. Aizpurua, "Strain effects on the electronic structure of strongly coupled self-assembled InAs/GaAs quantum dots: Tight-binding approach," Phys. Rev. B, vol. 74, pp. 195339195349, 2006.

[30] J. Kim, L.-W. Wang, and A. Zunger, "Comparison of the electronic structure of InAs/GaAs pyramidal quantum dots with different facet orientations," Phys. Rev. B, vol. 57, pp. R9408-R9411, 1998.

[31] A. J. Williamson and A. Zunger, "InAs quantum dots: Predicted electronic structure of free-standing versus GaAs-embedded structures," Phys. Rev. B, vol. 59, pp. 15819-15824, 1999.

[32] J. M. Jancu, R. Scholz, F. Beltram, and F. Bassani, "Empirical spds* tightbinding calculation for cubic semiconductors: General method and material parameters," Phys. Rev. B, vol. 57, pp. 6493-6507, 1998.

[33] G. Bester, S. Nair, and A. Zunger, "Pseudopotential calculation of the excitonic fine structure of million-atom self-assembled In1-xGaxAs/GaAs quantum dots," Phys. Rev. B, vol. 67, pp. 161306(R)-161309(R), 2003. 
[34] O. Gunawan, H. S. Djie, and B. S. Ooi, "Electronics states of interdiffused quantum dots," Phys. Rev. B, vol. 71, pp. 205319-205328, 2005.

[35] C. Y. Ngo, S. F. Yoon, W. J. Fan, and S. J. Chua, "Effects of size and shape on electronic states of quantum dots," Phys. Rev. B, vol. 74, pp. 245331$245340,2006$.

[36] R. A. Morrow and K. R. Brownstein, "Model effective-mass Hamiltonians for abrupt heterojunctions and the associated wave-function-matching conditions," Phys. Rev. B, vol. 30, pp. 678-680, 1984.

[37] H. Jiang and J. Singh, "Strain distribution and electronic spectra of InAs/GaAs self-assembled dots: An eight-band study," Phys. Rev. B, vol. 56, pp. 4696-4701, 1997.

[38] H. Fu, L.-W. Wang, and A. Zunger, "Applicability of the k·p method to the electronic structure of quantum dots," Phys. Rev. B, vol. 57, pp. 9971-9987, 1998.

[39] O. Stier, M. Grundmann, and D. Bimberg, "Electronic and optical properties of strained quantum dots modeled by 8-band k·p theory," Phys. Rev. B, vol. 59, pp. 5688-5701, 1999.

[40] N. Vukmirović, D. Indjin, V. D. Jovanovicacute, Z. Ikonić, and P. Harrison, "Symmetry of k·p Hamiltonian in pyramidal InAs/GaAs quantum dots: Application to the calculation of electronic structure," Phys. Rev. B, vol. 72, pp. 075356-075365, 2005.

[41] A. Schliwa, M. Winkelnkemper, and D. Bimberg, "Impact of size, shape, and composition on piezoelectric effects and electronic properties of In $(\mathrm{Ga})$ As/GaAs quantum dots," Phys. Rev. B, vol. 76, pp. 205324-205340, 2007.

[42] C. Pryor, M. E. Pistol, and L. Samuelson, "Electronic structure of strained InP/Ga0.51In0.49P quantum dots," Phys. Rev. B, vol. 56, pp. 10404-10411, 1997.

[43] S.-F. Tsay, J.-C. Chiang, Z. M. Chau, and I. Lo, "k·p finite-difference method:Band structures and cyclotron resonances of Al_ $\{x\} \mathrm{Ga} \_\{1-$ x \} Sb/InAs quantum wells," Phys. Rev. B, vol. 56, pp. 13242-13251, 1997.

[44] L. C. Lew Yan Voon and M. Willatzen, The $k \cdot p$ method: Electronic properties of semiconductors. Heidelberg: Springer Berlin Heidelberg, 2009.

[45] R. H. Parmenter, "Symmetry properties of the energy bands of the zinc blende structure," Phys. Rev., vol. 100, pp. 573-579, 1955.

[46] G. Dresselhaus, "Spin-orbit coupling effects in zinc blende structures," Phys. Rev., vol. 100, pp. 580-586, 1955.

[47] S. R. White and L. J. Sham, "Electronic Properties of Flat-Band Semiconductor Heterostructures," Phys. Rev. Lett., vol. 47, pp. 879-883, 1981.

[48] D. L. Smith and C. Mailhiot, "k·p theory of semiconductor superlattice electronic structure. I. Formal results," Phys. Rev. B, vol. 33, pp. 8345-8359, 1986.

[49] R. Winkler and U. Rössler, "General approach to the envelope-function approximation based on a quadrature method," Phys. Rev. B, vol. 48, pp. 8918-8927, 1993.

[50] F. Szmulowicz, "Numerically stable Hermitian secular equation for the envelope-function approximation for superlattices," Phys. Rev. B, vol. 54, pp. 11539-11547, 1996. 
[51] M. F. H. Schuurmans and G. W. T Hooft, "Simple calculations of confinement states in a quantum well," Phys. Rev. B, vol. 31, pp. 80418048, 1985.

[52] R. Eppenga, M. F. H. Schuurmans, and S. Colak, "New k·p theory for GaAs/Ga1-xA1xAs-type quantum wells," Phys. Rev. B, vol. 36, pp. 15541564, 1987.

[53] K. I. Kolokolov, J. Li, and C. Z. Ning, "k·p Hamiltonian without spuriousstate solutions," Phys. Rev. B, vol. 68, pp. 1613081-1613084, 2003.

[54] E. Tsuchida and M. Tsukada, "Electronic-structure calculations based on the finite-element method," Phys. Rev. B, vol. 52, pp. 5573-5578, 1995.

[55] H. T. Johnson, L. B. Freund, C. D. Akyuz, and A. Zaslavsky, "Finite element analysis of strain effects on electronic and transport properties in quantum dots and wires," J. Appl. Phys., vol. 84, pp. 3714-3725, 1998.

[56] J. C. Yi and N. Dagli, "Finite-element analysis of valence-band structure and optical-properties of quantum-wire arrays on vicinal substrates," IEEE J. Quantum Elect., vol. 31, pp. 208-218, 1995.

[57] H. T. Johnson, V. Nguyen, and A. F. Bower, "Simulated self-assembly and optoelectronic properties of InAs/GaAs quantum dot arrays," J. Appl. Phys., vol. 92, pp. 4653-4663, 2002.

[58] M. Grundmann, O. Stier, and D. Bimberg, "InAs/GaAs pyramidal quantum dots: Strain distribution, optical phonons, and electronic structure," Phys. Rev. B, vol. 52, pp. 11969-11981, 1995.

[59] C. Pryor, "Eight-band calculations of strained InAs/GaAs quantum dots compared with one-, four-, and six-band approximations," Phys. Rev. B, vol. 57, pp. 7190-7195, 1998.

[60] T. Mei, "Fourier transform-based $\mathrm{k} \cdot \mathrm{p}$ method of semiconductor superlattice electronic structure," J. Appl. Phys., vol. 102, pp. 053708-053712, 2007.

[61] B. Lassen, R. V. N. Melnik, and M. Willatzen, "Spurious Solutions in the Multiband Effective Mass Theory Applied to Low Dimensional Nanostructures," Commun. Comput. Phys., vol. 6, pp. 699-729, 2009.

[62] X. Cartoixà, D. Z. Y. Ting, and T. C. McGill, "Numerical spurious solutions in the effective mass approximation," J. Appl. Phys., vol. 93, pp. 3974-3981, 2003.

[63] L. W. Wang, J. N. Kim, and A. Zunger, "Electronic structures of 110 faceted self-assembled pyramidal InAs/GaAs quantum dots," Phys. Rev. B, vol. 59, pp. 5678-5687, 1999.

[64] L. Seravalli, M. Minelli, P. Frigeri, P. Allegri, V. Avanzini, and S. Franchi, "The effect of strain on tuning of light emission energy of InAs/InGaAs quantum-dot nanostructures," Appl. Phys. Lett., vol. 82, pp. 2341-2343, 2003.

[65] L. Seravalli, P. Frigeri, M. Minelli, P. Allegri, V. Avanzini, and S. Franchi, "Quantum dot strain engineering for light emission at 1.3, 1.4 and $1.5 \mu \mathrm{m}$," Appl. Phys. Lett., vol. 87, pp. 063101-0631003, 2005.

[66] L. Seravalli, M. Minelli, P. Frigeri, S. Franchi, G. Guizzetti, M. Patrini, T. Ciabattoni, and M. Geddo, "Quantum dot strain engineering of InAs/InGaAs nanostructures," J. Appl. Phys., vol. 101, pp. 024313-024320, 2007.

[67] A. D. Andreev, J. R. Downes, D. A. Faux, and E. P. O'Reilly, "Strain distributions in quantum dots of arbitrary shape," J. Appl. Phys., vol. 86, pp. 297-305, 1999. 
[68] S. Lee, O. L. Lazarenkova, P. von Allmen, F. Oyafuso, and G. Klimeck, "Effect of wetting layers on the strain and electronic structure of InAs selfassembled quantum dots," Phys. Rev. B, vol. 70, pp. 125307-125313, 2004.

[69] R. V. N. Melnik and M. Willatzen, "Bandstructures of conical quantum dots with wetting layers," Nanotechnology, vol. 15, pp. 1-8, 2004.

[70] B. A. Foreman, "Effective-mass Hamiltonian and boundary conditions for the valence bands of semiconductor microstructures," Phys. Rev. B, vol. 48, pp. 4964-4967, 1993.

[71] B. A. Foreman, "Elimination of spurious solutions from eight-band $\mathrm{k} \cdot \mathrm{p}$ theory," Phys. Rev. B, vol. 56, pp. R12748-R12751, 1997.

[72] E. P. Pokatilov, V. A. Fonoberov, V. M. Fomin, and J. T. Devreese, "Development of an eight-band theory for quantum dot heterostructures," Phys. Rev. B, vol. 64, pp. 245328-245343, 2001.

[73] R. G. Veprek, S. Steiger, and B. Witzigmann, "Ellipticity and the spurious solution problem of $\mathrm{k} \cdot \mathrm{p}$ envelope equations," Phys. Rev. B, vol. 76, pp. 165320-165328, 2007.

[74] R. G. Veprek, S. Steiger, and B. Witzigmann, "Operator ordering, ellipticity and spurious solutions in $\mathrm{k} \cdot \mathrm{p}$ calculations of III-nitride nanostructures," Opt. Quant. Electron., vol. 40, pp. 1169-1174, 2008.

[75] W. Yang and K. Chang, "Origin and elimination of spurious solutions of the eight-band k·p theory," Phys. Rev. B, vol. 72, pp. 233309-233312, 2005.

[76] A. T. Meney, B. Gonul, and E. P. O'Reilly, "Evaluation of various approximations used in the envelope-function method," Phys. Rev. B, vol. 50, pp. 10893-10904, 1994.

[77] B. A. Foreman, "Choosing a basis that eliminates spurious solutions in $\mathrm{k} \cdot \mathrm{p}$ theory," Phys. Rev. B, vol. 75, pp. 235331-235344, 2007.

[78] C. H. Lee, Y. J. Hong, Y. J. Kim, J. Yoo, H. Baek, S. R. Jeon, S. J. Lee, and G. C. Yi, "GaN/ZnO Nanotube Heterostructure Light-Emitting Diodes Fabricated on Si," IEEE J. Sel. Top. Quant., vol. 17, pp. 966-970, 2011.

[79] C. Y. Cho, I. K. Park, M. K. Kwon, J. Y. Kim, S. J. Park, D. R. Jung, and K. W. Kwon, "InGaN/GaN multiple quantum wells grown on microfacets for white-light generation," Appl. Phys. Lett., vol. 93, 2008.

[80] Z. L. Fang, "White emission by self-regulated growth of $\mathrm{InGaN} / \mathrm{GaN}$ quantum wells on in situ self-organized faceted n-GaN islands," Nanotechnology, vol. 22, 2011.

[81] S. Nagarajan, T. S. Oh, M. S. Kumar, C. H. Hong, and E. K. Suh, "Structural and optical properties of in-rich InAlGaN/InGaN heterostructures for white light emission," Jpn. J. Appl. Phys., vol. 47, pp. 4413-4416, 2008.

[82] D. Xiao, K. W. Kim, S. M. Bedair, and J. M. Zavada, "Design of white light-emitting diodes using InGaN/AlInGaN quantum-well structures," Appl. Phys. Lett., vol. 84, pp. 672-674, 2004.

[83] M. T. Hardy, D. F. Feezell, S. P. DenBaars, and S. Nakamura, "Group IIInitride lasers: a materials perspective," Mater. Today, vol. 14, pp. 408-415, 2011.

[84] H. Yoshida, M. Kuwabara, Y. Yamashita, K. Uchiyama, and H. Kan, "The current status of ultraviolet laser diodes," Phys. Status Solidi A, vol. 208, pp. 1586-1589, 2011.

[85] Z. W. Yang, R. Li, Q. Y. Wei, T. Yu, Y. Z. Zhang, W. H. Chen, and X. D. $\mathrm{Hu}$, "Analysis of optical gain property in the InGaN/GaN triangular shaped 
quantum well under the piezoelectric field," Appl. Phys. Lett., vol. 94, pp. 061120-061122, 2009.

[86] R. Cingolani, A. Botchkarev, H. Tang, H. Morkoc, G. Traetta, G. Coli, M. Lomascolo, A. Di Carlo, F. Della Sala, and P. Lugli, "Spontaneous polarization and piezoelectric field in GaN/A10.15Ga0.85N quantum wells: Impact on the optical spectra," Phys. Rev. B, vol. 61, pp. 2711-2715, 2000.

[87] L. H. Peng, C. W. Chuang, and L. H. Lou, "Piezoelectric effects in the optical properties of strained InGaN quantum wells," Appl. Phys. Lett., vol. 74, pp. 795-797, 1999.

[88] Y. L. Li, R. Huang, and Y. H. Lai, "Efficiency droop behaviors of InGaN/GaN multiple-quantum-well light-emitting diodes with varying quantum well thickness," Appl. Phys. Lett., vol. 91, pp. 181113-181115, 2007.

[89] P. M. Tu, C. Y. Chang, S. C. Huang, C. H. Chiu, J. R. Chang, W. T. Chang, D. S. Wuu, H. W. Zan, C. C. Lin, H. C. Kuo, and C. P. Hsu, "Investigation of efficiency droop for InGaN-based UV light-emitting diodes with InAlGaN barrier," Appl. Phys. Lett., vol. 98, pp. 211107-211109, 2011.

[90] S. H. Park, D. Ahn, and J. W. Kim, "Optical gain in InGaN/InGaAlN quantum well structures with zero internal field," Appl. Phys. Lett., vol. 92, pp. 171115-171117, 2008.

[91] H. Teisseyre, T. Suski, S. P. Lepkowski, S. Anceau, P. Perlin, P. Lefebvre, L. Konczewicz, H. Hirayama, and Y. Aoyagi, "Determination of built-in electric fields in quaternary InAlGaN heterostructures," Appl. Phys. Lett., vol. 82, pp. 1541-1543, 2003.

[92] S. H. Park, H. M. Kim, and D. Ahn, "Optical gain in GaN quantum well lasers with quaternary AlInGaN barriers," Jpn. J. Appl. Phys., vol. 44, pp. 7460-7463, 2005.

[93] M. Kneissl, D. W. Treat, M. Teepe, N. Miyashita, and N. M. Johnson, "Continuous-wave operation of ultraviolet InGaN/InAlGaN multiplequantum-well laser diodes," Appl. Phys. Lett., vol. 82, pp. 2386-2388, 2003.

[94] R. M. Martin, Electronic structure: basic theory and practical methods. Cambridge: Cambridge University Press, 2004.

[95] N. W. Ashcroft and N. D. Mermin, Solid state physics. New York: Holt, Rinehart and Winston, 1976.

[96] J. Callaway, Quantum theory of the solid state. Boston: Academic Press, 1991.

[97] G. Bastard, "Superlattice band structure in the envelope-function approximation," Phys. Rev. B, vol. 24, pp. 5693-5697, 1981.

[98] G. Bastard, "Theoretical investigations of superlattice band structure in the envelope-function approximation," Phys. Rev. B, vol. 25, pp. 7584-7597, 1982.

[99] G. Bastard and J. A. Brum, "Electronic states in semiconductor heterostructures," IEEE J. Quantum Elect., vol. 22, pp. 1625-1644, 1986.

[100] M. G. Burt, " An exact formulation of the envelope function method for the determination of electronic states in semiconductor microstructures," Semicond. Sci. Technol., vol. 2, pp. 460-462, 1987.

[101] R. I. Taylor and M. G. Burt, "Continuity conditions for envelope functions at semiconductor interfaces derived from the Bastard model," Semicond. Sci. Technol., vol. 2, pp. 485-490, 1987. 
[102] M. G. Burt, "The justification for applying the effective-mass approxiamtion to microstructures," J.Phys.-Condens. Mat., vol. 4, pp. 6651-6690, 1992.

[103] M. G. Burt, "Direct derivation of effective mass equations for microstructures with atomically abrupt boundaries," Phys. Rev. B, vol. 50, pp. 7518-7525, 1994.

[104] M. G. Burt, "Fundamentals of envelope function theory for electronic states and photonic modes in nanostructures," J.Phys.-Condens. Mat., vol. 11, pp. R53-R83, 1999.

[105] J. F. Nye, Physical properties of crystals: their representation by tensors and matrices. Oxford: Oxford University Press, 1985.

[106] S. L. Chuang and C. S. Chang, "A band-structure model of strained quantum-well wurtzite semiconductors," Semicond. Sci. Technol., vol. 12, pp. 252-263, 1997.

[107] T. B. Bahder, "Eight-band k·p model of strained zinc-blende crystals," Phys. Rev. B, vol. 41, pp. 11992-12001, 1990.

[108] W. F. Cady, Piezoeletricity. New York: McGraw-Hill, 1946.

[109] I. Vurgaftman, J. R. Meyer, and L. R. Ram-Mohan, "Band parameters for III-V compound semiconductors and their alloys," J. Appl. Phys., vol. 89, pp. 5815-5875, 2001.

[110] G. Bester, X. Wu, D. Vanderbilt, and A. Zunger, "Importance of SecondOrder Piezoelectric Effects in Zinc-Blende Semiconductors," Phys. Rev. Lett., vol. 96, pp. 187602-187605, 2006.

[111] G. Bester, A. Zunger, X. Wu, and D. Vanderbilt, "Effects of linear and nonlinear piezoelectricity on the electronic properties of InAs / GaAs quantum dots," Phys. Rev. B, vol. 74, pp. 081305-081308, 2006.

[112] J. Pal, G. Tse, V. Haxha, M. A. Migliorato, and S. Tomic, "Second-order piezoelectricity in wurtzite III-N semiconductors," Phys. Rev. B, vol. 84, pp. 085211-085217, 2011.

[113] "Group III nitride semiconductor compounds," in Physics and applications, B. Gil, Ed., ed. Oxford: Clarendon, 1998.

[114] H. Morkoc, Nitride semiconductors and devices. Berlin: Springer, 1999.

[115] F. Bernardini, V. Fiorentini, and D. Vanderbilt, "Spontaneous polarization and piezoelectric constants of III-V nitrides," Phys. Rev. B, vol. 56, pp. 10024-10027, 1997.

[116] A. D. Andreev and E. P. O'Reilly, "Theory of the electronic structure of GaN/AIN hexagonal quantum dots," Phys. Rev. B, vol. 62, pp. 1585115870, 2000.

[117] J. Singh, Electronic and optoelectronic properties of semiconductor structures. Cambridge: Cambridge University Press, 2003.

[118] J. Downes and D. A. Faux, "Calculation of strain distributions in multiplequantum-well strained-layer structures," J. Appl. Phys., vol. 77, pp. 24442447, 1995.

[119] M. Tadić, F. M. Peeters, K. L. Janssens, M. Korkusinski, and P. Hawrylak, "Strain and band edges in single and coupled cylindrical InAs/GaAs and InP/InGaP self-assembled quantum dots," J. Appl. Phys., vol. 92, pp. 58195829, 2002.

[120] P. N. Keating, "Effect of Invariance Requirements on the Elastic Strain Energy of Crystals with Application to the Diamond Structure," Phys. Rev., vol. 145, pp. 637-645, 1966. 
[121] C. Pryor, J. Kim, L. W. Wang, A. J. Williamson, and A. Zunger, "Comparison of two methods for describing the strain profiles in quantum dots," J. Appl. Phys., vol. 83, pp. 2548-2554, 1998.

[122] M. A. Cusack, P. R. Briddon, and M. Jaros, "Electronic structure of InAs/GaAs self-assembled quantum dots," Phys. Rev. B, vol. 54, pp. R2300-R2303, 1996.

[123] J. R. Downes, D. A. Faux, and E. P. Oreilly, "A simple method for calculating strain distributions in quantum dot structures," J. Appl. Phys., vol. 81, pp. 6700-6702, 1997.

[124] G. S. Pearson and D. A. Faux, "Analytical solutions for strain in pyramidal quantum dots," J. Appl. Phys., vol. 88, pp. 730-736, 2000.

[125] D. A. Faux and G. S. Pearson, "Green's tensors for anisotropic elasticity: Application to quantum dots," Phys. Rev. B, vol. 62, pp. R4798-R4801, 2000.

[126] D. A. Faux and U. M. E. Christmas, "Real-space Green's tensors for stress and strain in crystals with cubic anisotropy," J. Appl. Phys., vol. 98, 2005.

[127] T. Benabbas, P. Francois, Y. Androussi, and A. Lefebvre, "Stress relaxation in highly strained InAs/GaAs structures as studied by finite element analysis and transmission electron microscopy," J. Appl. Phys., vol. 80, pp. 2763-2767, 1996.

[128] J. D. Eshelby, "The determination of the elastic field of an ellipsoidal inclusion, and related problems," Proc. R. Soc. London, Ser. A, vol. 241, pp. 376-396, 1957.

[129] A. D. Prins and D. J. Dunstan, "A determination of relative bulk moduli of GaInAsP and InP," Philos. Mag. Lett., vol. 58, pp. 37-44, 1988.

[130] D. L. Smith and C. Mailhiot, "Piezoelectric effects in strained-layer superlattices," J. Appl. Phys., vol. 63, pp. 2717-2719, 1988.

[131] U. M. E. Christmas, A. D. Andreev, and D. A. Faux, "Calculation of electric field and optical transitions in InGaN/GaN quantum wells," J. Appl. Phys., vol. 98, 2005.

[132] D. P. Williams, A. D. Andreev, E. P. O'Reilly, and D. A. Faux, "Derivation of built-in polarization potentials in nitride-based semiconductor quantum dots," Phys. Rev. B, vol. 72, pp. 235318-235327, 2005.

[133] A. Bykhovski, B. Gelmont, and M. Shur, "The influence of the straininduced electric field on the charge distribution in GaN-AlN-GaN structure," J. Appl. Phys., vol. 74, pp. 6734-6739, 1993.

[134] S. H. Park and S. L. Chuang, "Comparison of zinc-blende and wurtzite GaN semiconductors with spontaneous polarization and piezoelectric field effects," J. Appl. Phys., vol. 87, pp. 353-364, 2000.

[135] A. D. Bykhovski, B. L. Gelmont, and M. S. Shur, "Elastic strain relaxation and piezoeffect in GaN-AlN, GaN-AlGaN and GaN-InGaN superlattices," J. Appl. Phys., vol. 81, pp. 6332-6338, 1997.

[136] N. Grandjean, B. Damilano, S. Dalmasso, M. Leroux, M. Laugt, and J. Massies, "Built-in electric-field effects in wurtzite $\mathrm{AlGaN} / \mathrm{GaN}$ quantum wells," J. Appl. Phys., vol. 86, pp. 3714-3720, 1999.

[137] S. H. Park, "Crystal orientation effects on electronic properties of wurtzite GaN/AlGaN quantum wells with spontaneous and piezoelectric polarization," Jpn. J. Appl. phys., vol. 39, pp. 3478-3482, 2000. 
[138] J. J. Shi and Z. Z. Gan, "Effects of piezoelectricity and spontaneous polarization on localized excitons in self-formed InGaN quantum dots," $J$. Appl. Phys., vol. 94, pp. 407-415, 2003.

[139] S. H. Park and S. L. Chuang, "Spontaneous polarization effects in wurtzite GaN/AlGaN quantum wells and comparison with experiment," Appl. Phys. Lett., vol. 76, pp. 1981-1983, 2000.

[140] S. H. Park and S. L. Chuang, "Piezoelectric effects on electrical and optical properties of wurtzite GaN/AlGaN quantum well lasers," Appl. Phys. Lett., vol. 72, pp. 3103-3105, 1998.

[141] P. O. Löwdin, "A note on the quantum-mechanical perturbation theory," $J$. Chem. Phys., vol. 19, pp. 1396-1401, 1951.

[142] J. M. Luttinger, "Quantum theory of cyclotron resonance for degerate bands," Phys. Rev., vol. 98, pp. 1560-1560, 1955.

[143] J. M. Luttinger and W. Kohn, "Motion of electrons and holes in perturbed periodic fields," Phys. Rev., vol. 97, pp. 869-883, 1955.

[144] E. O. Kane, "The k·p method " in Semiconductors and Semimetals. New York: Academic Press, 1967, p. 75.

[145] J. Bardeen, "An improved calculation of the energies of metallic Li and Na," J. Chem. Phys., vol. 6, pp. 367-371, 1938.

[146] F. Seitz, Modern theory of solids. New York: McGraw-Hill, 1940.

[147] W. Shockley, "Energy band structures in semiconductors," Phys. Rev., vol. 78, pp. 173-174, 1950.

[148] G. Dresselhaus, A. F. Kip, and C. Kittel, "Cyclotron resonance of electrons and holes in silicon and germanium crystals," Phys. Rev., vol. 98, pp. 368384, 1955.

[149] E. O. Kane, "Energy band structrue in p-type germanium and silicon," $J$. Phys. Chem. Solids, vol. 1, pp. 82-99, 1956.

[150] E. O. Kane, "Band structure of indium antimonide," J. Phys. Chem. Solids, vol. 1, pp. 249-261, 1957.

[151] G. E. Pikus and G. L. Bir, "Effect of deformation on the hole energy spectrum of germanium and silicon," Sov. Phys. Solid state, vol. 1, pp. 1502-1517, 1960.

[152] G. L. Bir and G. E. Pikus, Symmetry and strain-induced effects in semiconductors. New York: Wiely, 1974.

[153] T. B. Bahder, "Erratum: Eight-band $\mathrm{k} \cdot \mathrm{p}$ model of strained zinc-blende crystals [Phys. Rev. B 41, 11992 (1990)]," Phys. Rev. B, vol. 46, pp. 99139913, 1992.

[154] T. B. Bahder, "Analytic dispersion relations near the Gamma point in strained zinc-blende crystals," Phys. Rev. B, vol. 45, p. 1629, 1992.

[155] Y. Zhang, "Motion of electrons in semiconductors under inhomogeneous strain with apllication to laterally confined quantum-wells," Phys. Rev. B, vol. 49, pp. 14352-14366, 1994.

[156] P. Enders, A. Bärwolff, M. Woerner, and D. Suisky, "k p p theory of energy bands, wave functions, and optical selection rules in strained tetrahedral semiconductors," Phys. Rev. B, vol. 51, pp. 16695-16704, 1995.

[157] T. E. Ostromek, "Evaluation of matrix elements of the $8 \times 8 \mathrm{k} \cdot \mathrm{p}$ Hamiltonian with k-dependent spin-orbit contributions for the zinc-blende structure of GaAs," Phys. Rev. B, vol. 54, pp. 14467-14479, 1996. 
[158] G. A. Baraff and D. Gershoni, "Eigenfunction-expansion method for solving the quantum-wire problem: Formulation," Phys. Rev. B, vol. 43, pp. 4011-4022, 1991.

[159] D. Gershoni, C. H. Henry, and G. A. Baraff, "Calculating the opticalproperties of multidimensional heterostructures: Application to the modeling of quternary quantum-well lasers," IEEE J. Quantum Elect., vol. 29, pp. 2433-2450, 1993.

[160] S. L. Chuang and C. S. Chang, "k·p method for strained wurtzite semiconductors," Phys. Rev. B, vol. 54, pp. 2491-2504, 1996.

[161] X. Cartoixà, "Theoretical Methods for Spintronics in Semiconductors with Applications," Dorctor of Philosphy, Californian Instritute of Technology, Pasadena, 2003.

[162] M. Holm, M. E. Pistol, and C. Pryor, "Calculations of the electronic structure of strained InAs quantum dots in InP," J. Appl. Phys., vol. 92, pp. 932-936, 2002.

[163] H. Mohamed, Dilute Nitride Semiconductors. London: Elsevier, 2005.

[164] M. S. Skolnick and D. J. Mowbray, "Self-assembled semiconductor quantum dots: Fundamental physics and device a tipplications," Annu. Rev. Mater. Res., vol. 34, pp. 181-218, 2004.

[165] V. M. Ustinov, A. E. Zhukov, A. R. Kovsh, S. S. Mikhrin, N. A. Maleev, B. V. Volovik, Y. G. Musikhin, Y. M. Shernyakov, E. Y. Kondat'eva, M. V. Maximov, A. F. Tsatsul'nikov, N. N. Ledentsov, Z. I. Alferov, J. A. Lott, and D. Bimberg, "Long-wavelength quantum dot lasers on GaAs substrates," Nanotechnology, vol. 11, pp. 397-400, 2000.

[166] M. Henini and M. Bugajski, "Advances in self-assembled semiconductor quantum dot lasers," Microelectron. J., vol. 36, pp. 950-956, 2005.

[167] M. S. Skolnick and D. J. Mowbray, "Recent developments in the physics and applications of self-assembled quantum dots," Physica E, vol. 21, pp. 155-163, 2004.

[168] T. Hakkarainen, J. Toivonen, H. Koskenvaara, M. Sopanen, and H. Lipsanen, "Structural and optical properties of GaInNAs/GaAs quantum structures," J.Phys.-Condens. Mat., vol. 16, pp. S3009-S3026, 2004.

[169] P. Hazdra, J. Oswald, V. Komarnitskyy, K. Kuldová, A. Hospodková, J. Vyskočil, E. Hulicius, and J. Pangrác, "InAs/GaAs quantum dot structures emitting in the $1.55 \mu \mathrm{m}$ band," IOP Conf. Series: Mat. Sci. and Eng., vol. 6, pp. 012007-012010, 2009.

[170] M. Fischer, D. Bisping, B. Marquardt, and A. Forchel, "High-temperature continuous-wave operation of GaInAsN-GaAs quantum-dot laser diodes beyond 1.3 mu m," IEEE Photonics Technol. Lett., vol. 19, pp. 1030-1032, 2007.

[171] Q. Gong, R. Notzel, P. J. van Veldhoven, T. J. Eijkemans, and J. H. Wolter, "Wavelength tuning of InAs quantum dots grown on InP (100) by chemical-beam epitaxy," Appl. Phys. Lett., vol. 84, pp. 275-277, 2004.

[172] H. Saito, K. Nishi, and S. Sugou, "Ground-state lasing at room temperature in long-wavelength InAs quantum-dot lasers on $\operatorname{InP}(311) B$ substrates," Appl. Phys. Lett., vol. 78, pp. 267-269, 2001.

[173] F. Lelarge, B. Dagens, J. Renaudier, R. Brenot, A. Accard, F. van Dijk, D. Make, O. Le Gouezigou, J. G. Provost, F. Poingt, J. Landreau, O. Drisse, E. Derouin, B. Rousseau, F. Pommereau, and G. H. Duan, "Recent advances on InAs/InP quantum dash based, semiconductor lasers and optical 
amplifiers operating at $1.55 \mu \mathrm{m}, "$ IEEE J. Sel. Top. Quant., vol. 13, pp. 111-124, 2007.

[174] C. Paranthoen, N. Bertru, O. Dehaese, A. Le Corre, S. Loualiche, B. Lambert, and G. Patriarche, "Height dispersion control of InAs/InP quantum dots emitting at $1.55 \mathrm{mu}$ m," Appl. Phys. Lett., vol. 78, pp. 1751$1753,2001$.

[175] E. Homeyer, R. Piron, F. Grillot, O. Dehaese, K. Tavernier, E. Mace, A. Le Corre, and S. Loualiche, "First demonstration of a $1.52 \mathrm{mu} \mathrm{m}$ RT InAs/InP(311)B laser with an active zone based on a single QD layer," Semicond. Sci. Technol., vol. 22, pp. 827-830, 2007.

[176] F. Lelarge, B. Rousseau, B. Dagens, F. Poingt, E. Pommereau, and A. Accard, "Room temperature continuous-wave operation of buried ridge stripe lasers using InAs-InP (100) quantum dots as active core," IEEE Photonics Technol. Lett., vol. 17, pp. 1369-1371, 2005.

[177] J. S. Kim, J. H. Lee, S. U. Hong, W. S. Han, H. S. Kwack, C. W. Lee, and D. K. Oh, "Room-temperature operation of InP-based InAs quantum dot laser," IEEE Photonics Technol. Lett., vol. 16, pp. 1607-1609, 2004.

[178] C. G. Vandewalle, "Band lineups and deformation potentials in the modelsolid theory," Phys. Rev. B, vol. 39, pp. 1871-1883, 1989.

[179] S. Ridene, M. Debbichi, A. Ben Fredj, M. Said, and H. Bouchriha, "Energy-band structure and optical gain in strained $\ln \mathrm{As}(\mathrm{N}) / \mathrm{GaSb} / \ln \mathrm{As}(\mathrm{N})$ quantum well lasers," J. Appl. Phys., vol. 104, 2008.

[180] W. W. Chow and S. w. Koch, Semiconductor-Laser Fundamentals. Berlin: Springer, 1999.

[181] W. W. Chow, A. Knorr, S. Hughes, A. Girndt, and S. W. Koch, "Carrier correlation effects in a quantum-well semiconductor laser medium," IEEE J. Sel. Top. Quant., vol. 3, pp. 136-141, 1997.

[182] S. L. Chuang, "Optical gain of strained wurtzite GaN quantum-well lasers," IEEE J. Quantum Elect., vol. 32, pp. 1791-1800, 1996.

[183] C. S. Chang, S. L. Chuang, J. R. Minch, W. C. W. Fang, Y. K. Chen, and T. TanbunEk, "Amplified spontaneous emission spectroscopy in strained quantum-well lasers," IEEE J. Sel. Top. Quant., vol. 1, pp. 1100-1107, 1995.

[184] S. L. Chuang, J. Ogorman, and A. F. J. Levi, "Amplified spontaneous emission and carrier pinning in laser diodes "IEEE J. Quantum Elect., vol. 29, pp. 1631-1639, 1993.

[185] Y. C. Yeo, T. C. Chong, M. F. Li, and W. J. Fan, "Analysis of optical gain and threshold current density of wurtzite $\mathrm{InGaN} / \mathrm{GaN} / \mathrm{AlGaN}$ quantum well lasers," J. Appl. Phys., vol. 84, pp. 1813-1819, 1998.

[186] J. Minch, S. H. Park, T. Keating, and S. L. Chuang, "Theory and experiment of In1-xGaxAsyP1-y and In1-x-yGaxAlyAs long-wavelength strained quantum-well lasers," IEEE J. Quantum Elect., vol. 35, pp. 771$782,1999$.

[187] S. T. Ng, W. J. Fan, Y. X. Dang, and S. F. Yoon, "Comparison of electronic band structure and optical transparency conditions of $\operatorname{In}(\mathrm{x}) \mathrm{Ga}(1-$ $\mathrm{x}) \operatorname{As}(1-\mathrm{y}) \mathrm{N}(\mathrm{y}) / \mathrm{GaAs}$ quantum wells calculated by 10-band, 8-band, and 6band k center dot p models," Phys. Rev. B, vol. 72, 2005.

[188] S. W. Chang and S. L. Chuang, "Theory of optical gain of Ge-SixGeySn1x-y quantum-well lasers," IEEE J. Quantum Elect., vol. 43, pp. 249-256, 2007. 
[189] Y. H. Zhu, H. Y. Yu, and W. J. Fan, "Band structures and optical gain of strained GaAs(x)P(1-x-y)N(y)/GaP quantum wells," Appl. Phys. Lett., vol. 98, pp. 121112-121114, 2011.

[190] Y. H. Zhu, Q. Xu, W. J. Fan, and J. W. Wang, "Theoretical gain of strained $\mathrm{GeSn}(0.02) / \mathrm{Ge}(1-\mathrm{x}-\mathrm{y}$ ')Si(x)Sn(y ') quantum well laser," J. Appl. Phys., vol. $107,2010$.

[191] W. J. Fan, M. F. Li, T. C. Chong, and J. B. Xia, "Valence hole subbands and optical gain spectra of GaN/Ga1-xAlxN strained quantum wells," $J$. Appl. Phys., vol. 80, pp. 3471-3478, 1996.

[192] D. Gershoni, C. H. Henry, and G. A. Baraff, "Calculating the optical properties of multidimensional heterostructures: Application to the modeling of quaternary quantum well lasers " IEEE J. Quantum Elect., vol. 29, pp. 2433-2450, 1993.

[193] W. Liu, D. H. Zhang, W. J. Fan, X. Y. Hou, and Z. M. Jiang, "Intersubband transitions in InGaAsN/GaAs quantum wells," J. Appl. Phys., vol. 104, 2008 .

[194] K. Boujdaria, S. Ridene, and G. Fishman, "Luttinger-like parameter calculations," Phys. Rev. B, vol. 63, pp. 235302-235307, 2001.

[195] L. E. Brus, "A simple model for the ionization potential, electron affinity, and aqueous redox potentials of small semiconductor crystallites," J. Chem. Phys., vol. 79, pp. 5566-5571, 1983.

[196] L. E. Brus, "Electron-electron and electron-hole interactions in small semiconductor crystallites: The size dependence of the lowest excited electronic state," J. Chem. Phys., vol. 80, pp. 4403-4409, 1984.

[197] C. Cornet, C. Platz, P. Caroff, J. Even, C. Labbe, H. Folliot, A. Le Corre, and S. Loualiche, "Approach to wetting-layer-assisted lateral coupling of InAs/InP quantum dots," Phys. Rev. B, vol. 72, pp. 035342-035346, 2005.

[198] J. H. Park, J. Kirch, L. J. Mawst, C. C. Liu, P. F. Nealey, and T. F. Kuech, "Controlled growth of InGaAs/InGaAsP quantum dots on InP substrates employing diblock copolymer lithography," Appl. Phys. Lett., vol. 95, pp. 113111-113113, 2009.

[199] L. Seravalli, P. Frigeri, G. Trevisi, and S. Franchi, "1.59 mu m room temperature emission from metamorphic InAs/InGaAs quantum dots grown on GaAs substrates," Appl. Phys. Lett., vol. 92, pp. 213104-213106, 2008.

[200] I. Vurgaftman and J. R. Meyer, "Band parameters for nitrogen-containing semiconductors," J. Appl. Phys., vol. 94, pp. 3675-3696, 2003.

[201] R. Eppenga, "The Electronic and Optical Properties of GaAs/AlGaAs Quantum wells," TU Delft, Delft University of Technology, Delft, 1988.

[202] C. K. Williams, T. H. Glisson, J. R. Hauser, and M. A. Littlejohn, "Energy bandgap and lattice-constant contours of III-V quaternary alloys of the form AxByCzD or ABxCyDz," J. Electron. Mater., vol. 7, pp. 639-646, 1978.

[203] G. Martin, A. Botchkarev, A. Rockett, and H. Morkoc, "Valence-band discontinuities of wurtzite GaN, AlN, and InN heterojunctions measured by x-ray photoemission spectroscopy," Appl. Phys. Lett., vol. 68, pp. 25412543, 1996.

[204] S. H. Park and D. Ahn, "Many-body effects on optical gain in strained hexagonal and cubic GaN/AIGaN quantum well lasers," Appl. Phys. Lett., vol. 71, pp. 398-400, 1997. 
[205] A. David and N. F. Gardner, "Droop in III-nitrides: Comparison of bulk and injection contributions," Appl. Phys. Lett., vol. 97, pp. 193508-193511, 2010.

[206] J. H. Son and J. L. Lee, "Numerical analysis of efficiency droop induced by piezoelectric polarization in InGaN/GaN light-emitting diodes," Appl. Phys. Lett., vol. 97, pp. 032109-032112, 2010.

[207] M. Zhang, P. Bhattacharya, and W. Guo, "InGaN/GaN self-organized quantum dot green light emitting diodes with reduced efficiency droop," Appl. Phys. Lett., vol. 97, 2010.

[208] E. Selcuk, G. J. Hamhuis, and R. Notzel, "Complex laterally ordered InGaAs and InAs quantum dots by guided self-organized anisotropic strain engineering on shallow- and deep-patterned GaAs (311)B substrates," $J$. Appl. Phys., vol. 102, pp. 094301-094305, 2007.

[209] J. Lee, Z. M. Wang, V. Yazdanpanah, E. S. Kim, S. M. Koo, and G. J. Salamo, "In(x)Ga(1-x)As quantum wire network-like and ordered checker board-like nanostructures on GaAs (311) by low In composition multi-layer stacking," Phys. Status Solidi A, vol. 208, pp. 47-51, 2011.

[210] A. Trellakis, A. T. Galick, A. Pacelli, and U. Ravaioli, "Iteration scheme for the solution of the two-dimensional Schrodinger-Poisson equations in quantum structures," J. Appl. Phys., vol. 81, pp. 7880-7884, 1997.

[211] I. H. Tan, G. L. Snider, L. D. Chang, and E. L. Hu, "A self-consistent solution of schrodinger-poisson equations using a nonuniform mesh," $J$. Appl. Phys., vol. 68, pp. 4071-4076, 1990.

[212] J. H. Luscombe, A. M. Bouchard, and M. Luban, "Electron confinement in quantum nanostructures: Self-consistent Poisson-Schrödinger theory," Phys. Rev. B, vol. 46, pp. 10262-10268, 1992. 